\title{
A search for muon neutrino and antineutrino disappearance with the Booster Neutrino Beam
}

Kendall Brianna McConnel Mahn

\author{
Submitted in partial fulfillment of the \\ requirements for the degree \\ of Doctor of Philosophy \\ in the Graduate School of Arts and Sciences
}

COLUMBIA UNIVERSITY

2009 
(C) 2009

Kendall B. M. Mahn

All Rights Reserved 


\begin{abstract}
A search for muon neutrino and antineutrino disappearance with the Booster Neutrino Beam
\end{abstract}

\author{
Kendall B. M. Mahn
}

This dissertation presents a search for $\nu_{\mu}$ and $\bar{\nu}_{\mu}$ disappearance with the MiniBooNE experiment in the $\Delta m^{2}$ region of a few $\mathrm{eV}^{2}$. Disappearance measurements in this oscillation region constrain sterile neutrino models and CPT violation in the lepton sector. Fits to the shape of the $\nu_{\mu}$ and $\bar{\nu}_{\mu}$ energy spectra reveal no evidence for disappearance in either mode. This is the first test of $\bar{\nu}_{\mu}$ disappearance between $\Delta m^{2}=0.1-10 \mathrm{eV}^{2}$. In addition, prospects for performing a joint analysis using the SciBooNE detector in conjunction with MiniBooNE are discussed. 


\section{Contents}

List of Figures $\quad$ v

List of Tables $\quad x$

$\begin{array}{ll}\text { Acknowledgments } & \text { xi }\end{array}$

1 Overview 1

2 Introduction to Neutrinos and Neutrino Oscillation 3

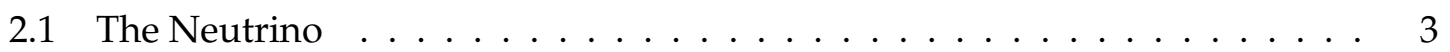

2.2 Neutrino Oscillation $\ldots \ldots \ldots \ldots \ldots \ldots$

2.2.1 Neutrino Oscillation Formalism _. . . . . . . . . . . . . . . . 4

2.2.2 Experimental Evidence for Neutrino Oscillation . . . . . . . . . 5

2.3 Neutrino Disappearance at a few $\mathrm{eV}^{2} \ldots \ldots \ldots \ldots \ldots$

3 The SciBooNE and MiniBooNE Experimental Setup 10

3.1 Neutrino Beams . . . . . . . . . . . . . . . . 10

3.2 The Booster Neutrino Beamline $(\mathrm{BNB}) \ldots \ldots \ldots \ldots$. . . . . . . . 11

3.3 The SciBooNE Detectors . . . . . . . . . . . . . . . . . . 15

3.3.1 The SciBar Detector . . . . . . . . . . . . . . . . . . . . . . . 15

3.3.2 The Electron Catcher $(\mathrm{EC}) \ldots \ldots \ldots \ldots$

3.3.3 The Muon Range Detector (MRD) . . . . . . . . . . . . . . . . . . 18

3.4 The MiniBooNE Detector . . . . . . . . . . . . . . . . . . . . . . . 21

3.5 Run Periods . . . . . . . . . . . . . . . . . . . . . . . . . . . . 29 
4 Predicting the Neutrino Rate 36

4.1 Neutrino Source . . . . . . . . . . . . . . . . 36

4.1 .1 Beamline Geometry . . . . . . . . . . . . . . . . 37

4.1 .2 Proton Beam . . . . . . . . . . . . . . . . 37

4.1 .3 Proton-Beryllium Interactions . . . . . . . . . . . . . . . . 37

$4.1 .4 \pi$ and $K$ Production $\ldots \ldots \ldots \ldots \ldots$

4.1 .5 Horn Magnetic Field . . . . . . . . . . . . . . . . . . . . . . 45

4.1 .6 Meson Decays to Neutrino Beam . . . . . . . . . . . . . . . . . . . . 49

4.1 .7 Neutrino and Antineutrino Fluxes . . . . . . . . . . . . . . . . . 49

4.2 Neutrino Interactions . . . . . . . . . . . . . . . . . . . . . . . 54

4.2 .1 CCQE Scattering . . . . . . . . . . . . . . 57

4.2.2 CC $\pi$ Production . . . . . . . . . . . . . . . . 63

4.2.3 Final State Internuclear Interactions (FSI) . . . . . . . . . . . . . 64

4.3 Detector Model . . . . . . . . . . . . . . . . . . . . 66

4.3 .1 SciBooNE Detector Model . . . . . . . . . . . . . . . . . 66

4.3.2 MiniBooNE Detector Model . . . . . . . . . . . . . . . . . . . . . 67

5 Event Selection and Reconstruction $\quad 70$

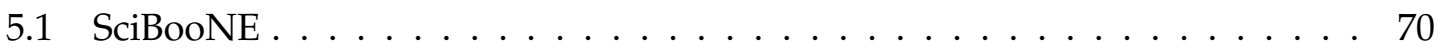

5.1 .1 SciBar Tracks $\ldots \ldots \ldots \ldots \ldots \ldots \ldots 71$

5.1 .2 SciBar-MRD Matched Tracks . . . . . . . . . . . . . . . . 71

5.1 .3 CC inclusive $\nu_{\mu}$ selection in SciBooNE . . . . . . . . . . . . . 72

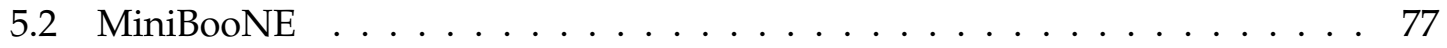

5.2.1 Selection of Beam-Induced Events . . . . . . . . . . . . . . 77

5.2.2 Reconstruction of Muon Events . . . . . . . . . . . . . . . 78

5.2.3 Data Quality Selection and Time Dependent Effects . . . . . . . . . 79

5.2.4 CCQE $\nu_{\mu}$ selection in MiniBooNE . . . . . . . . . . . . . 81

5.2 .5 CCQE $\bar{\nu}_{\mu}$ Selection . . . . . . . . . . . . . . . . . 82

5.2 .6 Neutrino Energy Calculation . . . . . . . . . . . . . 85

5.3 Comparison between MiniBooNE and SciBooNE samples . . . . . . . . . . . 89 
$6 \nu_{\mu}$ Disappearance with MiniBooNE $\quad 95$

6.1 Disappearance Search Method . . . . . . . . . . . . . . . . . 95

6.2 Producing Error Matrices $\ldots \ldots \ldots \ldots$

6.2.1 Shape and Normalization Error Matrices . . . . . . . . . . . 100

6.2 .2 Creating An Error Matrix . . . . . . . . . . . . . . . 100

6.3 Disappearance Fit Error Matrix . . . . . . . . . . . . . . . . . . . 102

6.3 .1 Flux Uncertainties . . . . . . . . . . . . . . . . . . . . . 102

6.3.2 Cross Section Uncertainties . . . . . . . . . . . . . . . . . . . . 104

6.3.3 Detector Uncertainties . . . . . . . . . . . . . . . . . . . . . . 104

6.3.4 Summary of Uncertainties . . . . . . . . . . . . . . . . . . . 106

6.4 Fit for $\nu_{\mu}$ Disappearance and Confidence Regions . . . . . . . . . . 106

6.4.1 Pearson's $\chi^{2}$ Method . . . . . . . . . . . . . . . . . . . . 110

$6.4 .2 \Delta \chi^{2}$ Method . . . . . . . . . . . . . . 112

6.4.3 Large Variations in Sensitivity Across $\Delta m^{2}$ aka "Wiggles" . . . . . . 120

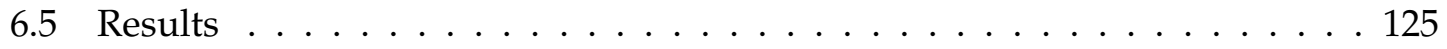

$6.5 .13+2$ Oscillation Models . . . . . . . . . . . . . . . . . . . 126

$7 \bar{\nu}_{\mu}$ Disappearance with MiniBooNE $\quad 130$

7.1 Systematic Uncertainties . . . . . . . . . . . . . . . . . . 130

7.2 Fit Method . . . . . . . . . . . . . . . . . . . . . . 134

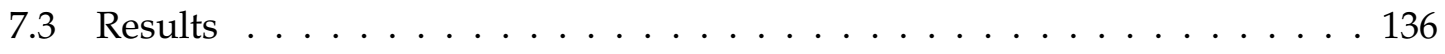

$8 \nu_{\mu}$ disappearance with MiniBooNE and SciBooNE 152

8.1 How Constraints Work . . . . . . . . . . . . . . . . . . . . . . . 154

8.1.1 Simple Two-Bin Cases . . . . . . . . . . . . . . . . . . . 154

8.2 Including A Near Detector in Disappearance Fits . . . . . . . . . . . 156

8.2.1 Systematic Uncertainties in SciBooNE and MiniBooNE . . . . . . . 156

8.2.2 Perfect Near Detector, Unoscillated . . . . . . . . . . . . . . . . 157

8.2.3 Perfect Near Detector, Oscillated . . . . . . . . . . . . . . . . . . 160

8.2.4 A Realistic Near Detector (SciBooNE) . . . . . . . . . . . . . . . . 160

8.3 Improvements to the Joint $\nu_{\mu}$ Analysis $\ldots \ldots \ldots \ldots$ 


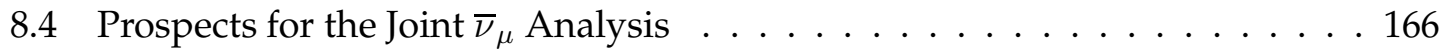

9 Conclusion

A MiniBooNE $E_{\nu}^{Q E}$ calculation $\quad 169$

B Shape-only Error Matrices 173

B.1 Shape-only Matrix Generation for Simple Cases . . . . . . . . . . . . . 173

B.1.1 Normalization Error Matrix . . . . . . . . . . . . . . . . . 173

B.1.2 Shape-only Error Matrix . . . . . . . . . . . . . . . . . . . . 174

B.1.3 Shape+Normalization Case . . . . . . . . . . . . . . . . 174

C Forming Error Matrices: Number of Draws 176

D Draft MiniBooNE $\nu_{\mu}$ and $\bar{\nu}_{\mu}$ Disappearance Publication 182

$\begin{array}{ll}\text { References } & 188\end{array}$ 


\section{List of Figures}

$2.13+1$ sterile neutrino mixing $\ldots \ldots \ldots \ldots \ldots \ldots$

2.2 MiniBooNE $\nu_{e}$ data and $3+2$ model predictions $\ldots \ldots \ldots$

3.1 A cartoon of the Booster Neutrino Beamline (BNB) at Fermilab. . . . . . . . . . 11

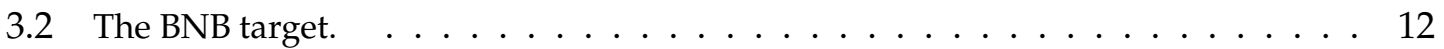

3.3 A engineering diagram of the BNB magnetic focusing horn. . . . . . . . . . 13

3.4 A engineering diagram of the $25 \mathrm{~m}$ absorber configuration. . . . . . . . . . . 14

3.5 The SciBar and Electron Calorimeter (EC) detectors . . . . . . . . . . . . . . . . 16

3.6 The Muon Range Detector (MRD) of SciBooNE. . . . . . . . . . . . . . . . . . . . 19

3.7 Moving the MRD into the SciBooNE detector hall. . . . . . . . . . . . . . . . 20

3.8 A typical event in SciBooNE. . . . . . . . . . . . . . . . . . . . . 22

3.9 A diagram the MiniBooNE detector and detector hall. . . . . . . . . . . . . . 23

3.10 The mounting of the PMTs within the MiniBooNE detector. . . . . . . . . . . . 23

3.11 A typical muon event in MiniBooNE. . . . . . . . . . . . . . . . . . 25

3.12 A schematic of the muon tracker and scintillation cubes. . . . . . . . . . 26

3.13 Reconstructed energy (tracker) versus reconstructed energy (fitter) . . . . . . . . . 27

3.14 Angular resolution of muons in MiniBooNE. . . . . . . . . . . . . . . 28

3.15 Extinction rate measurements of MiniBooNE oil. . . . . . . . . . . . . . . . 30

3.16 PMT hits relative to reconstructed direction for muons. . . . . . . . . . . . . . 31

3.17 Event rate per POT for neutrino candidates in MiniBooNE. . . . . . . . . . . . . 32

3.18 Event rate per POT for antineutrino candidates in MiniBooNE. . . . . . . . . 33

3.19 Event rate per POT for neutrino and antineutrino candidates SciBooNE. . . . . . . 35 
4.1 Hadronic cross section for beryllium . . . . . . . . . . . . . . . 38

$4.2 \pi^{+}$differential cross section for HARP data vs. $p_{\pi} \ldots \ldots \ldots \ldots \ldots$

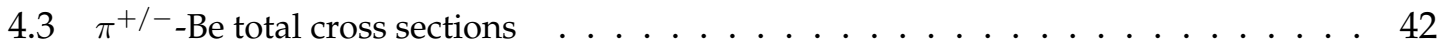

$4.4 \pi^{+/-}$-Be inelastic cross sections. . . . . . . . . . . . . . . . . . 43

$4.5 \pi^{+/-}$-Be quasi-elastic cross sections $\ldots \ldots \ldots \ldots \ldots \ldots \ldots$

$4.6 \pi^{+}$invariant cross section for HARP and E910 data. . . . . . . . . . . . . . . . 44

$4.7 K^{+}$production data and prediction versus $K^{+}$momentum $\ldots \ldots \ldots$. . . . . 46

4.8 Measurement of the magnetic field produced by the horn. . . . . . . . . . . . . 48

4.9 Horn current vs. MiniBooNE run number for neutrino mode. . . . . . . . . . . . 50

4.10 Horn current vs. MiniBooNE run number for antineutrino mode. . . . . . . . . . 51

4.11 Predicted neutrino mode flux at MiniBooNE . . . . . . . . . . . . . . . 52

4.12 Predicted neutrino flux at SciBooNE . . . . . . . . . . . . . . . . 52

4.13 Predicted antineutrino mode flux at MiniBooNE . . . . . . . . . . . . 53

4.14 Predicted antineutrino mode flux at SciBooNE . . . . . . . . . . . . . . . 53

4.15 Predicted flux at SciBooNE and MiniBooNE . . . . . . . . . . . . . . 54

4.16 Total neutrino $\mathrm{CC}$ cross section. $\ldots \ldots \ldots \ldots \ldots \ldots$

4.17 Total neutrino CC cross section. . . . . . . . . . . . . . . . 56

4.18 CCQE neutrino-nuclei scattering diagram $\ldots \ldots \ldots \ldots$

4.19 Effect of $M_{A}$ in $E_{\nu}$ compared to oscillations. . . . . . . . . . . . 60

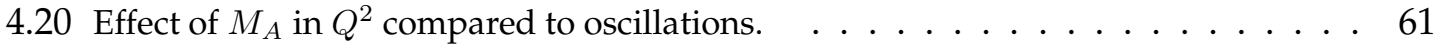

4.21 FSI cross section uncertainties. . . . . . . . . . . . . . . . . . . . . 65

4.22 Hit efficiency in a typical MRD plane . . . . . . . . . . . . . . 67

4.23 Pion interactions in SciBooNE. . . . . . . . . . . . . . . . . . 68

4.24 FSI detector uncertainties. . . . . . . . . . . . . . . . . . . . . 69

5.1 Neutrino parent momentum vs angle in SciBooNE $\ldots \ldots \ldots \ldots$. . . . . . . 74

5.2 Reconstructed $E_{\nu}^{Q E}$ vs. true $E_{\nu}$ for events in SciBooNE. . . . . . . . . . . . . . 75

$5.3 E_{\nu}^{Q E}$ resolution for CCQE SciBooNE events. $\ldots \ldots \ldots \ldots \ldots \ldots$

5.4 Average time $(\mathrm{ns})$ of the first subevent for all events. . . . . . . . . . . . . 77

5.5 Reconstructed versus true muon variables. . . . . . . . . . . . . . . 80 
5.6 Neutrino parent momentum vs angle in MiniBooNE . . . . . . . . . . . 83

5.7 Antineutrino sample parent particle momentum vs angle in MiniBooNE . . . . . . 84

5.8 Reconstructed $E_{\nu}^{Q E}$ vs. true $E_{\nu}$ for events in MiniBooNE. . . . . . . . . . 86

5.9 Ratio of data to prediction with and without energy corrections. . . . . . . . . . 87

$5.10 E_{\nu}^{Q E}$ resolution for CCQE events in the MiniBooNE neutrino sample. . . . . . . 88

5.11 Reconstructed $E_{\nu}^{Q E}$ vs. true $E_{\nu}$ for events in the MiniBooNE antineutrino sample. . 90

5.12 Pion parent kinematic overlap in MiniBooNE and SciBooNE . . . . . . . . . . 91

5.13 Kaon parent kinematic overlap in MiniBooNE and SciBooNE . . . . . . . . . . 92

$5.14 q^{2}$ overlap for CCQE and CC $\pi^{+}$events in MiniBooNE and SciBooNE . . . . . . . 93

5.15 Generated neutrino energy overlap for events in MiniBooNE and SciBooNE . . . . 94

6.1 Distortion of the neutrino energy spectrum due to disappearance. . . . . . . . . 97

6.2 Diagonal elements of the shape-only error matrix (MiniBooNE CCQE $\nu_{\mu}$ sample) . 108

6.3 Correlations the total shape-only error matrix for the MiniBooNE CCQE $\nu_{\mu}$ sample. 109

$6.4 \chi^{2}$ distribution for fake data throws with no underlying oscillation. . . . . . . . 111

6.5 MiniBooNE only $\nu_{\mu}$ disappearance sensitivity. . . . . . . . . . . . . . 113

6.6 Effect of errors on disappearance sensitivity. . . . . . . . . . . . . . . . . . 114

$6.7 \chi^{2}$ (best fit) distribution for fake data throws with no underlying oscillation. . . . . 116

$6.8 \Delta \chi^{2}$ distribution for fake data throws with no underlying oscillation. . . . . . . 117

6.9 Location of underlying signal vs. best fit point. . . . . . . . . . . . . 118

$6.10 \chi^{2}$ (best fit) distribution for fake data throws with no underlying oscillation. . . . . 119

6.11 Comparison of $\Delta \chi^{2}$ method and Pearson $\chi^{2}$ method. . . . . . . . . . . 121

6.12 Effect of $\Delta m^{2}$ vs. $E_{\nu}^{Q E}$ in MiniBooNE. . . . . . . . . . . . . 122

$6.13 \chi^{2}$ vs. $\Delta m^{2}$ in MiniBooNE. . . . . . . . . . . . . . . . . . . . 123

6.14 Effect of changing binning on $\chi^{2}$ vs. $\Delta m^{2}$ in MiniBooNE. . . . . . . . . . 124

$6.1590 \%$ CL exclusion of $\bar{\nu}_{e}$ disappearance (Bugey) . . . . . . . . . . . . . 125

$6.16 E_{\nu}^{Q E}$ distribution for MiniBooNE CCQE $\nu_{\mu}$ sample. . . . . . . . . . . . 127

6.17 MiniBooNE $\nu_{\mu}$ disappearance limit. . . . . . . . . . . . . . . . 128

7.1 Diagonal elements of the shape-only error matrix (MiniBooNE CCQE $\bar{\nu}_{\mu}$ sample) . 132

7.2 Correlations the total shape-only error matrix for the MiniBooNE CCQE $\bar{\nu}_{\mu}$ sample. 133 
7.3 MiniBooNE sensitivity to antineutrino disappearance (only antineutrinos oscillate) 134

7.4 Effect of errors on $\bar{\nu}_{\mu}$ disappearance sensitivity. . . . . . . . . . . . . . 135

7.5 Effect of normalization differences between neutrinos and antineutrinos on $E_{\nu}^{Q E} \quad .137$

7.6 Effect of relative normalization differences on the sensitivity. . . . . . . . . . 138

7.7 Effect of absorber plates on $E_{\nu}^{Q E} \ldots \ldots \ldots \ldots \ldots \ldots \ldots$

7.8 Data vs. $E_{\nu}^{Q E}$ for periods with no absorber present in the beamline . . . . . . . 140

7.9 Data (error bars) and unoscillated prediction $E_{\nu}^{Q E}$ for the 0 absorber configuration. 141

7.10 Data (error bars) and unoscillated prediction $E_{\nu}^{Q E}$ for the 1 absorber configuration. 142

7.11 Data (error bars) and unoscillated prediction $E_{\nu}^{Q E}$ for the 2 absorber configuration. 143

7.12 Ratio of data to unoscillated prediction $E_{\nu}^{Q E}$ for each absorber configuration. . . . 144

7.13 Antineutrino data and prediction vs. $E_{\nu}^{Q E} \ldots \ldots \ldots \ldots \ldots$. . . . . . 147

7.14 Antineutrino data fit assuming antineutrinos oscillate only. . . . . . . . . . . 148

7.15 Antineutrino fit to just zero absorber data assuming only antineutrinos oscillate. . 149

7.16 Antineutrino fit to one absorber data assuming only antineutrinos oscillate. . . . 150

7.17 Antineutrino fit to two absorber data assuming only antineutrinos oscillate. . . . . 151

8.1 Neutrino energy spectrum for SciBooNE, K2K, and T2K experiments . . . . 153

8.2 Diagonal elements of the shape-only error matrix (SciBooNE CC inclusive $\nu_{\mu}$ sample) 158

8.3 Correlations the total shape-only error matrix for MiniBooNE and SciBooNE . . . 159

8.4 Unoscillated near detector and far detector disappearance fit. . . . . . . . . . . . . 161

8.5 Oscillations at an ideal (MiniBooNE-like) near detector at $100 \mathrm{~m}$. . . . . . . . . 162

8.6 Oscillated near detector and far detector disappearance fit. . . . . . . . . . . . 163

8.7 Oscillations at SciBooNE compared to MiniBooNE. . . . . . . . . . . . . . . . 164

8.8 Joint MiniBooNE/SciBooNE preliminary $90 \%$ sensitivity . . . . . . . . . . . . 165

9.1 MiniBooNE $\nu_{\mu}$ and $\bar{\nu}_{\mu}$ disapparance $90 \%$ CL sensitivity and limit . . . . . . . . 168

A.1 Reconstructed vs. true muon kinetic energy $\ldots \ldots \ldots$. . . . . . . . . . . 170

A.2 Reconstructed $E_{\nu}^{Q E}$ vs. true $E_{\nu}$ after first correction. $\ldots \ldots \ldots \ldots$. . . . . 171

A.3 Reconstructed $E_{\nu}^{Q E}$-true $E_{\nu}$ vs. $Q^{2} \ldots \ldots \ldots \ldots \ldots \ldots \ldots \ldots \ldots \ldots \ldots \ldots$

A.4 Reconstructed $E_{\nu}^{Q E}$ vs. true $E_{\nu}$ after second correction $\ldots \ldots \ldots$. . . . . 172 
C.1 Fractional normalization of a matrix vs. number of draws . . . . . . . . . . 178

C.2 Fractional shape uncertainty and number of draws used. . . . . . . . . . . . . 179

C.3 $\chi^{2}$ as a function of number of draws. . . . . . . . . . . . . . . . . 181 


\section{List of Tables}

2.1 Direct mass measurements of the neutrino flavors. . . . . . . . . . . . . 4

3.1 Neutrino and antineutrino run periods on MiniBooNE. . . . . . . . . . . 34

3.2 Neutrino and antineutrino run periods on SciBooNE. . . . . . . . . . . . . 34

$4.1 \pi^{+}$differential cross section model parameters $\ldots \ldots \ldots \ldots$. . . . . . 40

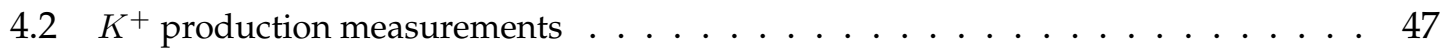

$4.3 K^{+}$production model parameters $\ldots \ldots \ldots \ldots \ldots$

5.1 Meson parents of the antineutrino sample . . . . . . . . . . . . . . . . 82

6.1 Neutrino energy bin delimiters . . . . . . . . . . . . . . . . . . . 98

6.2 Summary of systematic uncertainties $\left(\nu_{\mu}\right) \ldots \ldots \ldots \ldots$. . . . . . . . 107

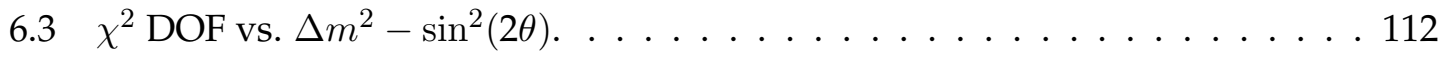

$6.4 \chi^{2}$ DOF vs. $\Delta m^{2}-\sin ^{2}(2 \theta) \ldots \ldots \ldots \ldots \ldots \ldots \ldots \ldots \ldots \ldots \ldots \ldots \ldots \ldots \ldots$

6.5 MiniBooNE CCQE $\nu_{\mu}$ data samples and prediction (I) . . . . . . . . . . 126

6.6 MiniBooNE CCQE $\nu_{\mu}$ data samples and prediction (II) . . . . . . . . . . . 126

6.7 Best fit 3+2 model parameter values and $\chi^{2}$ to MiniBooNE $\nu_{\mu}$ and $\bar{\nu}_{\mu}$ data. . . . . 129

7.1 Summary of systematic uncertainties $\left(\bar{\nu}_{\mu}\right) \ldots \ldots \ldots \ldots$. . . . . . . . 131

7.2 Data and unoscillated prediction in $E_{\nu}^{Q E}$ bins between $0 .-1.0 \mathrm{GeV}$. . . . . . . 145

7.3 Data and unoscillated prediction in $E_{\nu}^{Q E}$ bins between 1.1-1.9 GeV. . . . . . . . . 145

8.1 Summary of systematic uncertainties in joint analysis . . . . . . . . . . . . 157 


\section{Acknowledgments}

First of all, I would like to express my gratitude to Mike Shaevitz. Thank you for being an excellent adviser. I am extremely grateful for the constructive criticism, patience and wisdom you provide.

Second, I would like to thank the members of the MiniBooNE collaboration. Thank you, Janet Conrad and Bill Louis, the first spokespeople who also took time to help my analysis and progress. Steve Brice and Richard Van DeWater, thank you for your work as spokespeople, and your expertise in analysis and hardware. Byron Roe, thank you for a very thorough review of my analysis. Richard Imlay, thank you for the thoughtful crafting of the paper. Alexis, Dave, Jocelyn, Michel, Van and Georgia, it is always an honor to be called a Columbia student when I am in such good company. Teppei, Denis, Mike W., Bob, Ted, Ryan, Chris C., and Serge, thank you for letting me learn so much from you. Thank you, exquisite postdocs, Zelimir, Sam, Chris P., Jon L., Hray, Hiro, Martin, Terry, Zarko, Mitch, Jarek, and Bari. Thank you, all fellow MiniBooNErs, as there are many more who have shaped me over days or years.

Third, I would like to thank the members of the SciBooNE collaboration. Morgan Wascko and Tsuyoshi Nakaya have been excellent leaders and have kept us on an unbelievable schedule, thank you. Hide, you were an incredible example of patience and competence, thank you. Hayato-san, I hope someday to be as talented as you. To the incredible students of SciBooNE (Bancho, Nakaji, Yoshi, Takei-kun, Joan, Jose, Joe, Gary, Aaron), thank you. To Rob and Fermilab staff who made the MRD happen, thank you. Thank you, all members of SciBooNE, for a truly wonderful set of experiences proposing, building, commissioning and understanding the SciBooNE detectors and data.

Finally, I wish to thank my parents, two wonderful people who have been very sup- 
portive and loving. To my family, whether you be McDonald, McConnel, or Mahn, you are in my heart. To my best friend, Rebekah, thank you for 20 years of patient listening and love. Thank you, Nathan, for making me laugh, smile and happy every day. 


\section{Chapter 1}

\section{Overview}

In the last fifteen years, it has been discovered that neutrinos have mass, because of the observation of neutrino oscillations. Neutrino oscillations occur when one flavor of neutrino converts to another flavor and can only occur if there are small differences in the masses of the neutrinos. One signature of neutrino oscillation is neutrino "disappearance", i.e. if a known source of a particular flavor of neutrino, $\nu_{\alpha}$, oscillates into another (possibly unobserved) flavor of neutrino, $\nu_{\beta}$, then less $\nu_{\alpha}$ will be observed, as if they disappeared.

Neutrino oscillation has been observed consistent with three generations of neutrinos, but a third observation of oscillation by the LSND experiment suggests additional generation(s) of "sterile" neutrinos, observable only via neutrino oscillation. Alternate explanations of the LSND signal also include CPT violation, where neutrinos do not oscillate in the same way as antineutrinos.

The MiniBooNE experiment is capable of a search for $\nu_{\mu} \rightarrow \nu_{x}$ and $\bar{\nu}_{\mu} \rightarrow \bar{\nu}_{x}$ disappearance, a direct test of sterile neutrinos and CPT violation in the neutrino sector. A pure $\nu_{\mu}$ or $\bar{\nu}_{\mu}$ beam is produced at the Fermi National Accelerator Laboratory (Fermilab) and observed at a the MiniBooNE, a $~ 1$ kton Cherenkov detector $541 \mathrm{~m}$ from production. Disappearance is observable in MiniBooNE as distortion of the shape of the measured neutrino

(or antineutrino) energy spectrum. No disappearance is observed in a high statistics, high purity $\nu_{\mu}$ and $\bar{\nu}_{\mu}$ samples in MiniBooNE.

Over the period of time MiniBooNE took data, the SciBooNE detectors were placed 
into the beamline at $100 \mathrm{~m}$ to perform dedicated cross section measurements of neutrinos and antineutrinos. With the same neutrino target and beam, SciBooNE provides a constraint on the neutrino rate observed at MiniBooNE which can be used to improve the disappearance analysis.

This thesis describes a search for muon neutrino and muon antineutrino disappearance using the MiniBooNE detector, and the prospects for a joint disappearance measurement using both SciBooNE and MiniBooNE.

Chapter 2 describes the basic properties of the neutrino, and introduces neutrino oscillation and disappearance. Chapter 3 describes the neutrino source and the two experiments, MiniBooNE and SciBooNE, which observe the neutrino source. Chapter 4 describes how the beamline, neutrino interactions and detection of neutrinos are modeled, and Chapter 5 describes the selection and reconstruction of neutrino events in both detectors.

The last three chapters of this thesis are original work. In Chapter 6, neutrino events in MiniBooNE are compared to prediction and to a disappearance hypothesis to set a competitive limit on neutrino disappearance. This result excludes some $3+2$ sterile neutrino models. Chapter 7 is the analog of the previous chapter for antineutrino events in MiniBooNE and also sets a competitive limit. Finally, Chapter 8 details the combined disappearance analysis using events in MiniBooNE and SciBooNE, with prospects for both $\nu_{\mu}$ and $\bar{\nu}_{\mu}$ channels. 


\section{Chapter 2}

\section{Introduction to Neutrinos and Neutrino Oscillation}

\subsection{The Neutrino}

In 1930, Wolfgang Pauli proposed the existence of the neutrino to explain missing energy in the decay of the neutron. He lamented that he had proposed a particle which could never be detected, but in 1956, Reines and Cowen detected the (anti) neutrino at a nuclear reactor.

Since then, the properties of the neutrino $(\nu)$ and antineutrino $(\bar{\nu})$ have been studied experimentally.

- Neutrinos are neutral.

- There are three flavors of neutrinos: the electron neutrino $\left(\nu_{e}\right)$, the muon neutrino $\left(\nu_{\mu}\right)$ and the tau neutrino $\left(\nu_{\tau}\right)$. There are only three "light" flavors, because the decay of the $\mathrm{Z}$ boson constrains the number of neutrinos it can decay to [1].

- Experimentally, only the left handed neutrino (and right handed antineutrino) have been observed. A particle is left handed if the spin of the particle is is antiparallel to the direction the particle travels. Neutrinos are detectable only from weak interactions, and the weak force couples only to left handed particles (or right handed antiparticles) as it is a V-A (vector - axial vector) Lagrangian. 


\begin{tabular}{|c|c|}
\hline neutrino type & mass \\
\hline$\nu_{e}$ & $<2.5 \mathrm{eV}[2]$ \\
\hline$\nu_{\mu}$ & $<170 \mathrm{keV}[3]$ \\
\hline$\nu_{\tau}$ & $<18.2 \mathrm{MeV}[4]$ \\
\hline
\end{tabular}

Table 2.1: Direct mass measurements of the neutrino flavors

Direct mass measurements of the neutrino have only placed upper limits on the neutrino mass, as summarized in Table 2.1. Neutrinos are therefore very light particles (the electron's mass is $0.5 \mathrm{MeV}$ ), or they have no mass at all.

\subsection{Neutrino Oscillation}

In the last fifteen years, experiments have demonstrated that neutrinos do have small masses, by observing neutrino oscillation. This section describes what neutrino oscillation is, and the suite of experiments which demonstrates neutrinos oscillate.

\subsubsection{Neutrino Oscillation Formalism}

The neutrino mass eigenstates are related to the flavor states by a mixing matrix.

$$
\left|\nu_{i}\right\rangle=\sum_{i}^{3} U_{\alpha, i}\left|\nu_{\alpha}\right\rangle
$$

where $\left|\nu_{i}\right\rangle$ is the mass eigenstate $i$ of the neutrino, $\left|\nu_{\alpha}\right\rangle$ is the flavor eigenstate (with $\alpha=e, \mu, \tau)$ and $U_{\alpha, i}$ is the mixing matrix between flavor and mass states.

In the case of the $e, \mu, \tau$, the mass and flavor states are the same, so this matrix is the identity. In the case of the quarks, $U$ has large diagonal elements, and small off-diagonal elements. In the case of neutrinos, $U$ has large off-diagonal elements.

$U_{\alpha, i}$ can be factorized into three blocks:

$$
U_{\alpha, i}=\left(\begin{array}{ccc}
1 & 0 & 0 \\
0 & c\left(\theta_{23}\right) & s\left(\theta_{23}\right) \\
0 & -s\left(\theta_{23}\right) & c\left(\theta_{23}\right)
\end{array}\right)\left(\begin{array}{ccc}
c\left(\theta_{13}\right) & 0 & s\left(\theta_{13} e^{-i \delta}\right. \\
0 & 1 & 0 \\
-s\left(\theta_{13} e^{-i \delta}\right. & 0 & c\left(\theta_{13}\right)
\end{array}\right)\left(\begin{array}{ccc}
c\left(\theta_{12}\right) & s\left(\theta_{12}\right) & 0 \\
-s\left(\theta_{12}\right) & c\left(\theta_{12}\right) & 0 \\
0 & 0 & 1
\end{array}\right)
$$


where $c(\omega)$ stands for $\cos (\omega), s(\omega)$ stands for $\sin (\omega), \theta_{12}, \theta_{23}, \theta_{13}$ are the mixing angles and $\delta$ is a $\mathrm{CP}$ violating phase.

The first block corresponds to mixing between the $\nu_{\mu}$ and the $\nu_{\tau}$. Just considering this block as two neutrino mixing where:

$$
\nu_{\mu}(t)=\sum-\sin \left(\theta_{23}\right)\left|\nu_{2}\right\rangle-\cos \left(\theta_{23}\right)\left|\nu_{3}\right\rangle
$$

The probability to observe a $\nu_{\mu}$ after it has traveled a distance $\mathrm{L}(\mathrm{km})$ with energy $\mathrm{E}(\mathrm{GeV})$ is:

$$
P_{\nu_{\mu} \rightarrow \nu_{\mu}}=\left\langle\nu_{\mu} \mid \nu_{\mu}(t)\right\rangle=1-\sin ^{2}\left(2 \theta_{23}\right) \sin ^{2}\left(\frac{1.27 \Delta m^{2}{ }_{23} L}{E}\right)
$$

where $\Delta m_{23}^{2}=m_{3}^{2}-m_{2}^{2}$ is the mass splitting between mass eigenstates 2 and 3 . This formula is generic for any two neutrino mixing; the probability for oscillation for two neutrino mixing depends on the mixing angle $(\theta)$, the mass splitting between mass eigenstates $\mathrm{A}$ and $\mathrm{B}\left(\Delta m_{A B}^{2}\right)$, the neutrino energy and distance it has traveled. If all neutrino mass eigenstates had no mass, this probability would be zero, and neutrinos would not oscilate. For a source of $\nu_{\mu}$, this probability indicates that when $L / E$ is of the same size as $\Delta m^{2}{ }_{23}$, the $\nu_{\mu}$ will disappear, and less will be observed. Conversely, the $\nu_{\tau}$ which the $\nu_{\mu}$ oscillate into will appear in the pure $\nu_{\mu}$ beam. Therefore, neutrino oscillation is observable using the appearance of a new flavor and or the disappearance of the original flavor; appearance experiments detect the new flavor, and disappearance experiments look for a deficit of the original one.

\subsubsection{Experimental Evidence for Neutrino Oscillation}

Neutrino oscillation has been observed for three distinct $\Delta m^{2}$ regions, solar $\left(\Delta m^{2} \sim\right.$ $\left.10^{-3} \mathrm{eV}^{2}\right)$, atmospheric $\left(\Delta m^{2} \sim 10^{-3} \mathrm{eV}^{2}\right)$ and $\operatorname{LSND}\left(\Delta m^{2} \sim 1 \mathrm{eV}^{2}\right)$.

\subsubsection{Solar Neutrino Oscillation}

The Sun produces neutrinos as it burns $\left(p+p \rightarrow D+e^{+}+\nu_{e}+E\right)$. The number of neutrinos coming from the Sun can be predicted from the observed luminosity of the 
Sun, but initial calculations did not agree with what was observed by experiments measuring $\nu_{e}$. This discrepancy was explained using neutrino oscillation; the total number of active flavors from the Sun is correct, but the $\nu_{e}$ oscillate into $\nu_{\mu}$ and $\nu_{\tau}$. Solar neutrino experiments have shown that $\theta_{12} \approx 32^{\circ}$ and $\Delta m_{12}^{2} \sim 10^{-5} \mathrm{eV}^{2}[6,7,8,9,10,11,13,14]$.

\subsubsection{Atmospheric Neutrino Oscillation}

Neutrinos are also produced in the atmosphere. When cosmic rays hit the gas in the atmosphere, pions are produced which decay to neutrinos:

$$
\begin{aligned}
& \pi^{+} \rightarrow \mu^{+}+\nu_{\mu} \\
& \mu^{+} \rightarrow e^{+}+\nu_{e}+\bar{\nu}_{\mu}
\end{aligned}
$$

For each pion, a $\nu_{\mu}$ and a $\bar{\nu}_{\mu}$ are produced, as well as one $\nu_{e}$. An experiment measuring $\nu_{e}$ and $\nu_{\mu}$ would expect to see a ratio of 2:1, but instead, the Super-Kamiokande [15] experiment observed less $\nu_{\mu}$ and $\bar{\nu}_{\mu}$ than expected. This was later confirmed by the accelerator based K2K [16] and Minos [17] experiments, which observe $\nu_{\mu}$ disappearance consistent with "atmospheric" oscillation with $\Delta m_{23}^{2} \sim 10^{-3} \mathrm{eV}^{2}$ and $\theta_{23} \approx 45^{\circ}$.

\subsubsection{The LSND Experiment}

The LSND experiment observed an excess of $\bar{\nu}_{e}$ in a $\bar{\nu}_{\mu}$ beam [18], or $\nu_{e}$ appearance, corresponding to a third $\Delta m^{2}$ around $1 \mathrm{eV}^{2}$. A third independent $\Delta m^{2}$ indicates a fourth mass eigenstate, but the $\mathrm{Z}$ boson only decays to three light flavors as shown by the LEP experiments.

A solution to this problem was to introduce a "sterile" neutrino:

$$
\left|\nu_{s}\right\rangle=\sum_{i}^{4} U_{\alpha, i}^{*}\left|\nu_{i}\right\rangle
$$

where there are four mass eigenstates, and the new sterile flavor does not couple to the weak force or is too massive for the $\mathrm{Z}$ to decay to. Sterile neutrino models can add $1(3+1)$ or multiple, $\mathrm{N},(3+\mathrm{N})$ sterile neutrinos to the mixing matrix. 

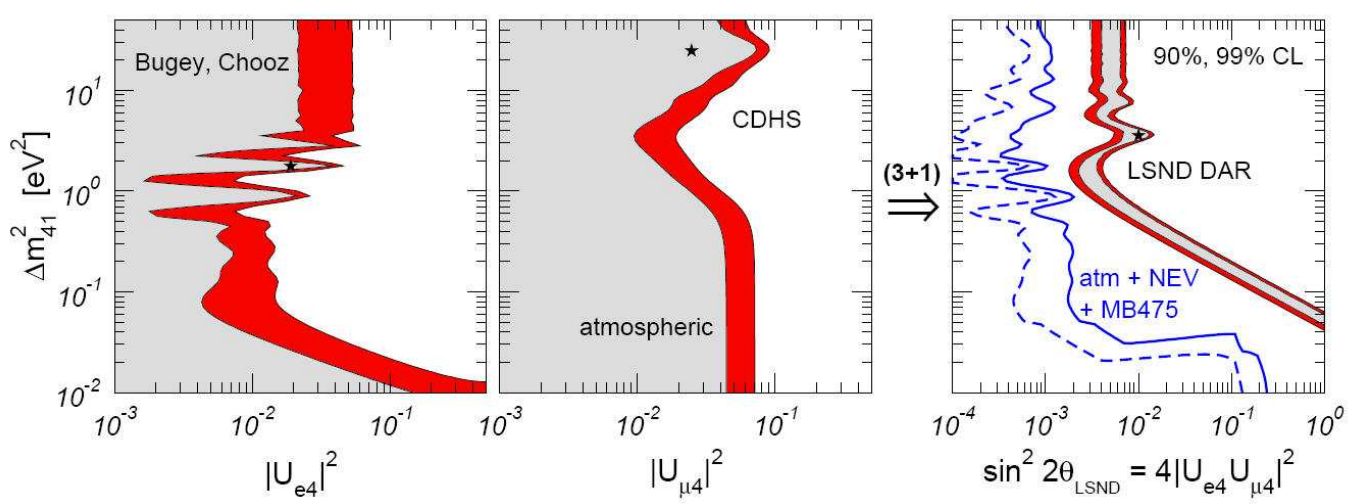

Figure 2.1: Allowed and excluded regions of sterile neutrino mixing. Mixing is characterized by a mass splitting between the sterile neutrino and the three active flavors $\left(\Delta m_{41}^{2}\right)$, and a mixing angle. In the case of $\bar{\nu}_{e} \rightarrow \nu_{x}$ disappearance, the mixing angle is $\sin ^{2}(\theta)=\left|U_{e 4}\right|^{2}$, in the case of $\nu_{\mu} \rightarrow \nu_{x}$, the mixing angle is $\sin ^{2}(\theta)=\left|U_{\mu 4}\right|^{2}$. Bounds on $\left|U_{e 4}\right|^{2}$ and $\left|U_{\mu 4}\right|^{2}$ are shown in the left and middle plots. The allowed 90\% (red) and 99\% (grey) for the LSND experiment are shown along with the exclusion region (right excluded) from the no-evidence-for-oscillation (NEV) experiments. This plot is from Ref. [24].

The MiniBooNE experiment was designed to test LSND, and, in 2007, the MiniBooNE experiment [19] excluded two neutrino appearance-only oscillation (98\% CL) as an explanation of the LSND excess, assuming CPT is not violated $\left(P_{\bar{\nu}_{\mu} \rightarrow \bar{\nu}_{e}}=P_{\nu_{\mu} \rightarrow \nu_{e}}\right)$.

\subsection{Neutrino Disappearance at a few $\mathrm{eV}^{2}$}

Disappearance searches $\left(\nu_{\mu} \rightarrow \nu_{x}\right)$ provide a complementary channel to appearance channels $\left(\nu_{\mu} \rightarrow \nu_{e}\right)$. In addition, disappearance searches are directly sensitive to oscillation to sterile neutrinos. Two disappearance searches at $\sim 1 \mathrm{eV}^{2}, \mathrm{CCFR}$ [20] and CDHS [21] excluded 3+1 sterile neutrino models [22] as an explanation of LSND before MiniBooNE's results. Figure 2.1 shows the bounds on $3+1$ mixing parameters, $\left|U_{\mu 4}\right|^{2}$ and $\left|U_{e 4}\right|^{2}$ and the LSND result, which is excluded with $\nu_{e}$ and $\nu_{\mu}$ disappearance data.

Remaining models which include more than one sterile neutrino $(3+2)$, sterile neu- 


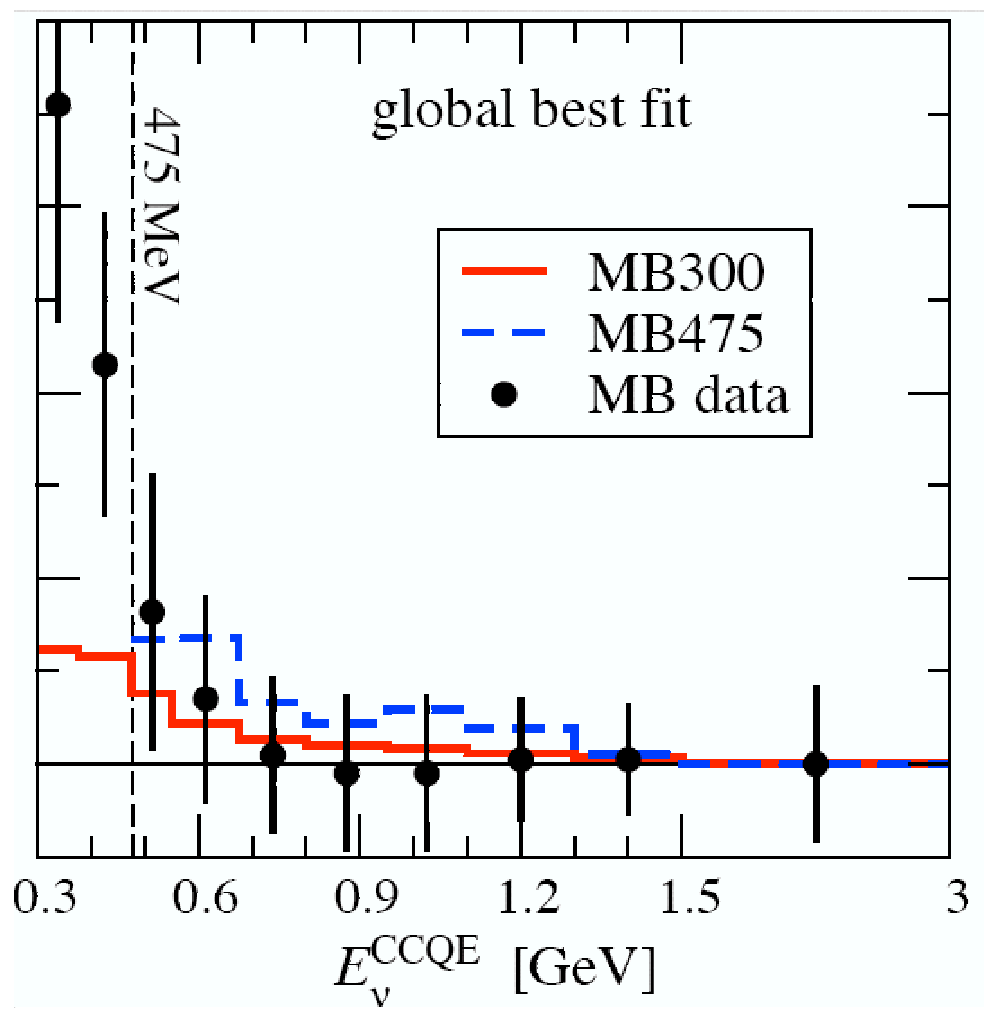

Figure 2.2: MiniBooNE $\nu_{e}$ data (black points) vs. reconstructed neutrino energy $\left(E_{\nu} C C Q E\right.$ $(\mathrm{GeV}))$ and 3+2 model best fit values from a global fit to all existing appearance and disappearance data (blue dash, red solid).

trinos in extra dimensions [27], decaying sterile neutrinos [28], and CPT violation [29] have all been proposed to explain LSND and MiniBooNE data simultaneously. Figure 2.2 shows two 3+2 models compared to MiniBooNE $\nu_{e}$ data, constructed from fits to all appearance and disappearance data, which reconcile the LSND excess with the MiniBooNE lack of a signal. However, $3+2$ models do not fit well to the observed excess at low energies in MiniBooNE data.

To probe for disappearance, this thesis will test for a general two-flavor mixing $\nu_{\mu} \rightarrow$ $\nu_{x}$ disappearance characterized by one large mass splitting $\left(\Delta m^{2} \equiv \Delta m_{h k}^{2}\right)$ between the light neutrino mass states $k$, which participate in standard three neutrino oscillation, and one oscillation amplitude $\sin ^{2}(2 \theta)=4\left|U_{\mu, h}\right|^{2}\left(1-\left|U_{\mu, h}\right|^{2}\right)$, where $\left|U_{\mu, h}\right|^{2}$ is the muon flavor content of the heavy state $h$. 
Two specific 3+2 sterile neutrino models are also tested from Ref. [23] and Ref. [25]. The two sterile neutrinos have masses $m_{4}$ and $m_{5}$ much larger than the light neutrinos $\sim m_{1}$. The probability for disappearance for $3+2$ models is:

$P_{\nu_{\mu} \rightarrow \nu_{\mu}}=1-4\left(\left[1-\left|U_{\mu 4}\right|^{2}-\left|U_{\mu 5}\right|^{2}\right]\left(\left|U_{\mu 4}\right|^{2} \sin ^{2} x_{41}+\left|U_{\mu 5}\right|^{2} \sin ^{2} x_{51}+\right)+\left|U_{\mu 4}\right|^{2}\left|U_{\mu 5}\right|^{2} \sin ^{2} x_{5} 4\right)$

where $x_{i j}=1.27 \Delta m_{i j}^{2} L / E$. While the entire $\Delta m_{41}^{2}, \Delta m_{51}^{2},\left|U_{\mu 4}\right|^{2}$ and $\left|U_{\mu 5}\right|^{2}$ parameter space is not explored, Ref. [23] and Ref. [25] fit 3+2 models to existing disappearance and appearance experiments and produce a best fit set of parameters for $3+2$ sterile neutrino models. These models [23, 25] favor relatively large mixing angles in the $\Delta m^{2}$ range of LSND to still explain LSND. The best fit values for 3+2 mixing are tested against the MiniBooNE $\nu_{\mu}$ and $\bar{\nu}_{\mu}$ energy spectrum.

Under CPT, $P_{\nu_{\mu} \rightarrow \nu_{\mu}}=P_{\bar{\nu}_{\mu} \rightarrow \bar{\nu}_{\mu}}$, so a difference between neutrino and antineutrino disappearance would indicate CPT violation. No measurement has been made of antineutrino disappearance below $10 \mathrm{eV}^{2}$, and this is uniquely probed by MiniBooNE. 


\section{Chapter 3}

\section{The SciBooNE and MiniBooNE Experimental Setup}

\subsection{Neutrino Beams}

To search for $\nu_{\mu}$ disappearance $\left(\nu_{\mu} \rightarrow \nu_{x}\right)$ requires as pure a source of $\nu_{\mu}$ as possible. Since the sun and reactors are predominantly $\nu_{e}$ or $\bar{\nu}_{e}$, and the flux of atmospheric neutrinos is prohibitively low, this means a man-made $\nu_{\mu}$ beam must be produced to study neutrino oscillation.

Accelerator facilities most commonly produce proton beams, but protons can also be used to create a neutrino beam. When a proton interacts with a nuclear target it produces mesons, typically pions and kaons. Pions and kaons both decay into neutrinos. Although neutrinos are neutral, if the parent particle is focused, the resulting neutrino beam will be too. Fig. 3.1 shows a cartoon of the relevant features of the neutrino beamline, including the SciBooNE and MiniBooNE detectors.

This chapter discusses the production and subsequent detection of neutrinos, starting with the accelerator protons and ending with the inner workings of the two neutrino detectors. 


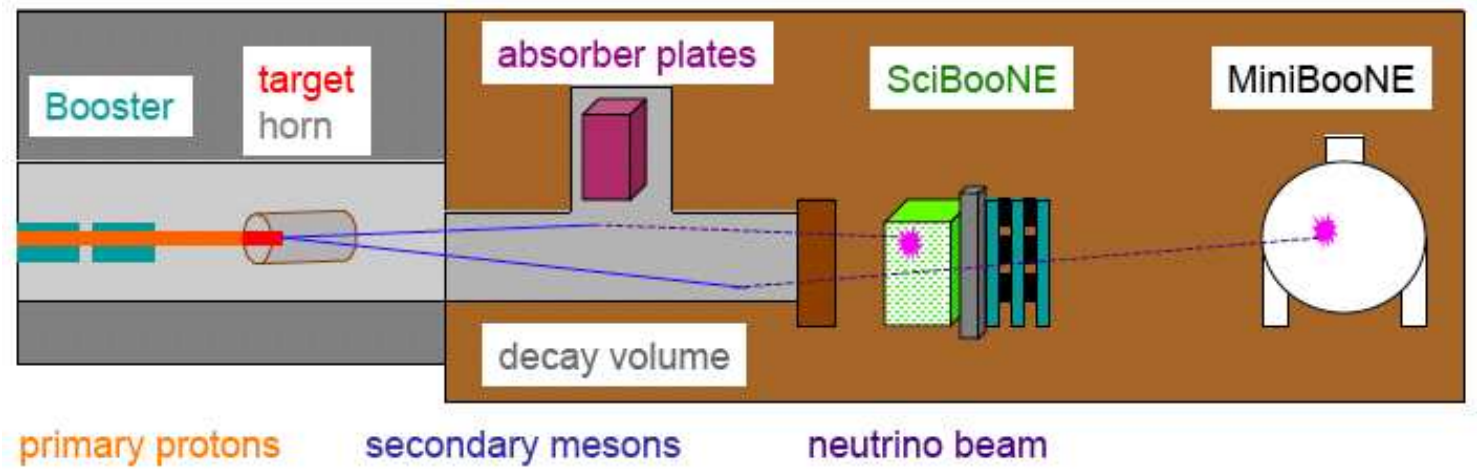

Figure 3.1: A cartoon (not to scale) of the neutrino beam used for both experiments. Protons from the booster hit a target inside a horn, the subsequent mesons decay and produce neutrinos detectable at SciBooNE and MiniBooNE.

\subsection{The Booster Neutrino Beamline (BNB)}

Fermi National Accelerator Laboratory (Fermilab) uses a chain of five accelerators to create the proton-anti proton collisions used in the discovery of the top quark; the neutrino beamline used for MiniBooNE and SciBooNE only uses the first three. Hydrogen gas is converted into $\mathrm{H}^{-}$ions with the Cockcroft-Walton generator and accelerated to $750 \mathrm{keV}$. The ions are then accelerated using alternating electromagnetic fields in a linear accelerator to $400 \mathrm{MeV}$. A thin carbon foil strips the electrons away, leaving only protons which enter the Booster synchrotron. For more details on the Fermilab accelerator complex, see Ref. [30].

The Booster accelerates protons to $8 \mathrm{GeV}(8.9 \mathrm{GeV} / \mathrm{c}$ momentum), and then sends the protons down the neutrino beamline. More details about the neutrino beamline construction can be found under Ref. [31]. A typical beam spill contains $4 \times 10^{12}$ protons over a period of $1.6 \mu \mathrm{s}$, and protons can be sent down the neutrino beamline at a maximum rate of $5 \mathrm{~Hz}$ (typically 2-4 Hz). The nature of the acceleration technique results in 81 smaller "bunches" within each beam spill, each $\sim 6$ ns wide, separated by 19 ns.

Protons are sent down the neutrino beamline and pass through a series of beam position monitors (BPMs) and toroids. The two toroids closest to the target (neutrino produc- 


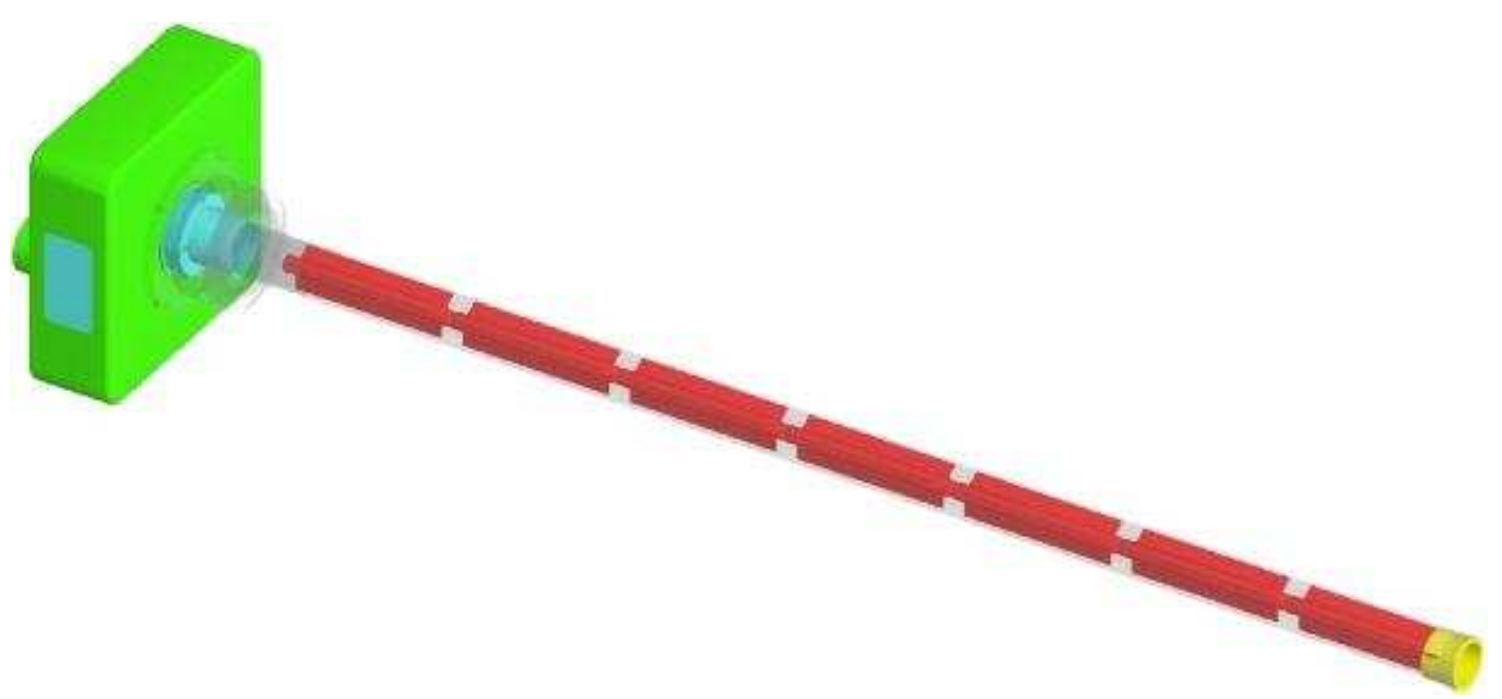

Figure 3.2: A engineering diagram of the BNB beryllium target. The proton beam enters from the left.

tion point) are called Toroid_860 and Toroid_875, based on their position in the beamline. These measure the total number of protons on target (POT); the position of the beam on the target, and its spread are also monitored before it hits the target. The total POT and the run periods for both SciBooNE and MiniBooNE can be found at the end of this chapter, Section 3.5. The toroids are calibrated every year and agree within $2 \%$ tolerance.

The protons hit a beryllium target situated inside a magnetic focusing horn. Beryllium (or carbon) targets are chosen for neutrino beams because low $\mathrm{Z}$ targets have a large interaction length relative to the radiation length, so the protons do not lose energy before interacting. The target is 1.7 interaction lengths long $(71 \mathrm{~cm})$ with a diameter of $1 \mathrm{~cm}$, and contains seven "slugs" of beryllium, as shown in Fig.3.2. Each slug is supported by three Be "fins" which extend outward and support the target within a sleeve. Air is circulated through the sleeve to keep the target cool, as the incident proton beam deposits a lot of heat.

Protons interacting with the target produce mesons, which are focused by a strong magnetic field produced by a conductor called a "horn". Current flows mainly on the surface of the aluminum horn creating a strong magnetic field between the outside and inner surfaces which focuses positively charged particles in neutrino mode running, and 


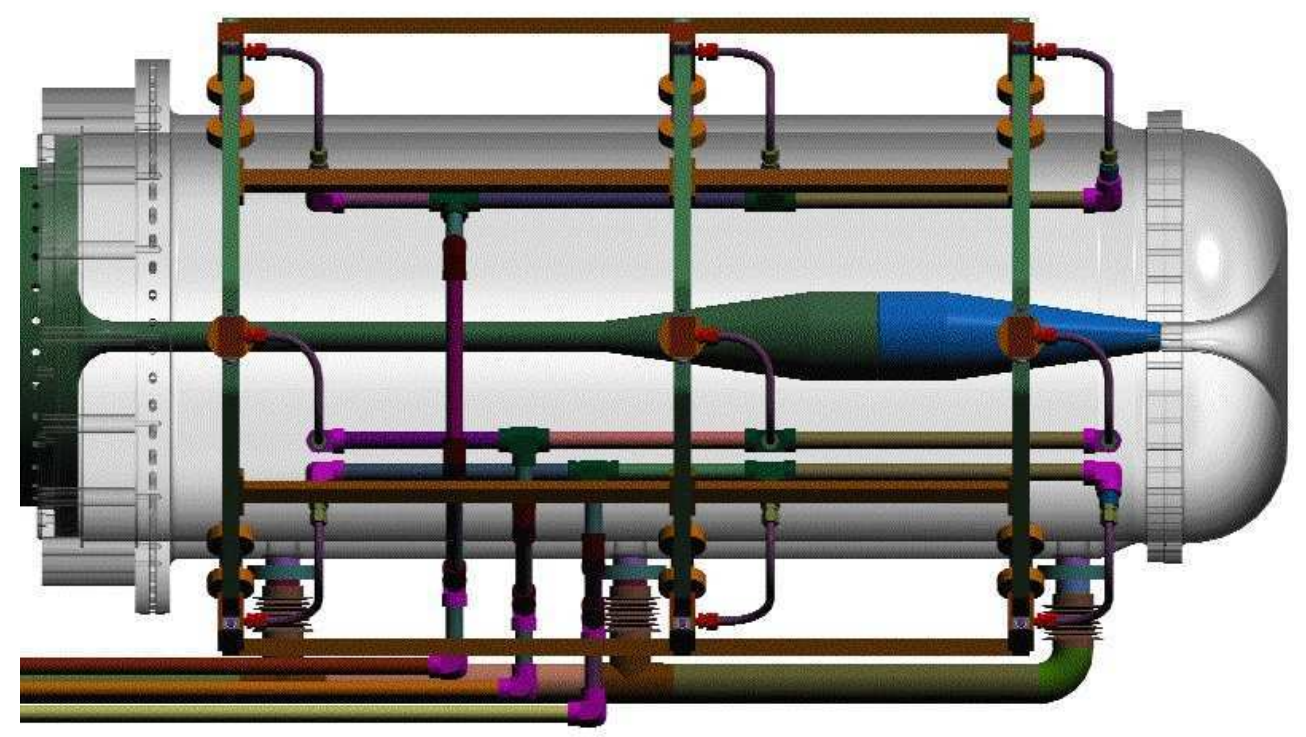

Figure 3.3: The BNB magnetic focusing horn. Current flows along the outer (grey) conductor to the inner conductor (dark green and blue) producing a strong magnetic field. The target sits inside the left portion of the inner conductor. The water cooling apparatus is also shown.

defocuses negatively charged ones. In antineutrino mode running, negatively charged mesons are focused. The magnetic horn used in the BNB is shown in Fig. 3.3. The use of a horn in the neutrino beam increases the flux of the parent particles directed at the detectors, which in turn increases the flux of the neutrino beam by approximately a factor of six. For more information on how horns work and their relationship to neutrino beams, see Ref. [32]. The horn is cooled by a closed water system. From the start of the MiniBooNE neutrino run in February 2003 until one month before the accelerator summer shutdown in July 2004, the first horn pulsed 96 million times before failing due to corrosion on the stripline. The second horn is still running, with record number of 306 million pulses as of March 2nd, 2009.

The mesons pass through a $60 \mathrm{~cm}$ collimator and enter a $50 \mathrm{~m}$ long, $182 \mathrm{~cm}$ diameter 


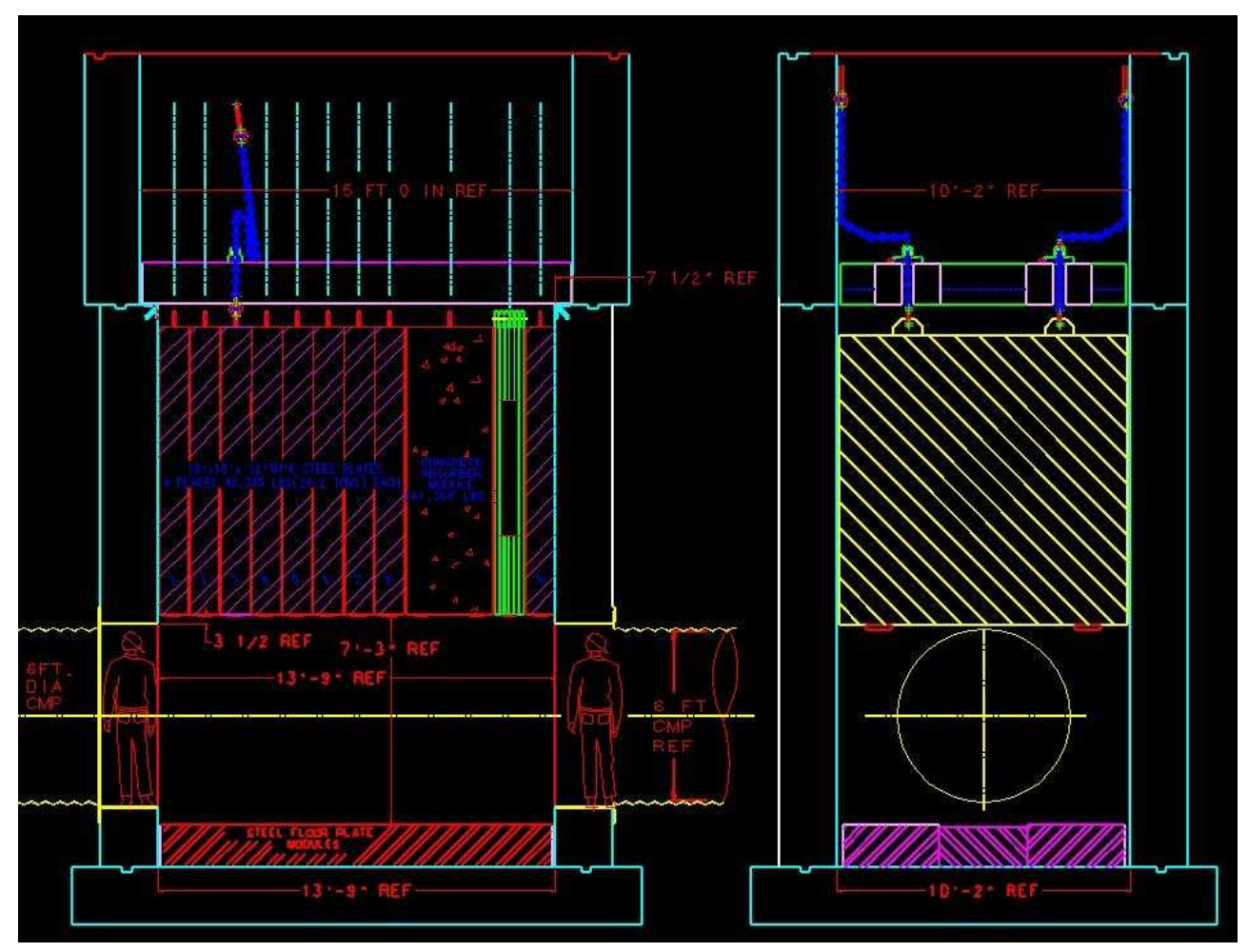

Figure 3.4: The $25 \mathrm{~m}$ absorber plate setup. Ten steel $\left(\sim 10^{\prime} \times 10^{\prime}\right)$ plates, depicted as red boxes with hash marks, are suspended 7' above the decay volume. Plate \# 1 is the first plate on the left, plate \# 10 is just downstream of a larger "block", which is a muon monitor.

decay volume filled with air. The mesons decay to charged leptons and neutrinos or are absorbed by the beam dump, a $3.8 \mathrm{~m}$ thick steel and concrete region at the end of the decay volume.

Suspended above the decay volume are ten steel absorber plates. Fig.3.4 shows the plate configuration above the decay volume. Each plate is 25 tons, with 6 individual 2" steel sheets welded together. These were intended to be deployed as a systematics check, as a shortened decay volume $(50 \mathrm{~m} \rightarrow 25 \mathrm{~m})$ will change the relative rate of pion, muon and kaon decays. However, it also absorbs the parent mesons, so the flux is reduced by approximately $10 \%$ for each plate inserted into the decay volume.

Over the 2006 accelerator shutdown, an absorber plane fell vertically into the beamline. On August 29th, 2006, a second plate also fell; this date is known due to changes 
in the rate and secondary beam radiation monitors known as "chipmunks". The chains which suspend the plates were made of hardened steel with a working load of 17 ton capable of supporting the 25 ton plates safely indefinitely. However, hardened steel is susceptible to stress corrosion cracking, and when the chains corroded due to the beamline radiation environment, the chains snapped, dropping the plates. During a subsequent shutdown, the plates were removed and re-secured above the beamline using stainless steel, which does not corrode like the hardened steel does.

The deployment of the plates resulted in three distinct time periods of running with 0, 1 or 2 absorbers in place. Based on the effect on the data over this running, it is believed that plate \#10 dropped first, and that plate \#7 dropped second.

\subsection{The SciBooNE Detectors}

The SciBooNE experiment is $100 \mathrm{~m}$ downstream of the target. SciBooNE consists of three detectors: a scintillating bar and fiber tracker (SciBar), an electron calorimeter (EC), and a muon range detector (MRD).

\subsubsection{The SciBar Detector}

SciBar stands for "scintillating bar detector" [33, 34, 35]. It consists of $1.3 \mathrm{~cm} \times 2.5 \mathrm{~cm} \times$ $300 \mathrm{~cm}$ rectangular extruded polystyrene scintillator (1\% PPO and $0.03 \%$ POPOP by weight) bars. 14,336 bars are glued together in alternating vertical and horizontal planes to create a $3 \mathrm{~m} \times 3 \mathrm{~m} \times 1.7 \mathrm{~m}$ volume weighing 15 tons, as shown in Fig. 3.5. Each bar has a $1.8 \mathrm{~mm}$ diameter hole which houses a single, $1.5 \mathrm{~mm}$ diameter wavelength shifting (WLS) fiber, and each bar is covered with co-extruded reflector material $\left(0.25 \mathrm{~mm}\right.$ thick, $15 \% \mathrm{TiO}_{2}$ infused polystyrene). A plane is 116 strips glued together, with 124 planes total. SciBar is 4.1 radiation lengths in the beam direction and 2.2 interaction lengths.

The main purpose of the fiber is to transport light out of the scintillator to the photomultiplier tube (PMT). The scintillator's attenuation length of less than $10 \mathrm{~cm}$ is untenable for such a large detector, but the fiber's attenuation length is much longer $(350 \mathrm{~cm})$. Groups of 64 fibers are brought out together on one side of the detector; the fibers are held 


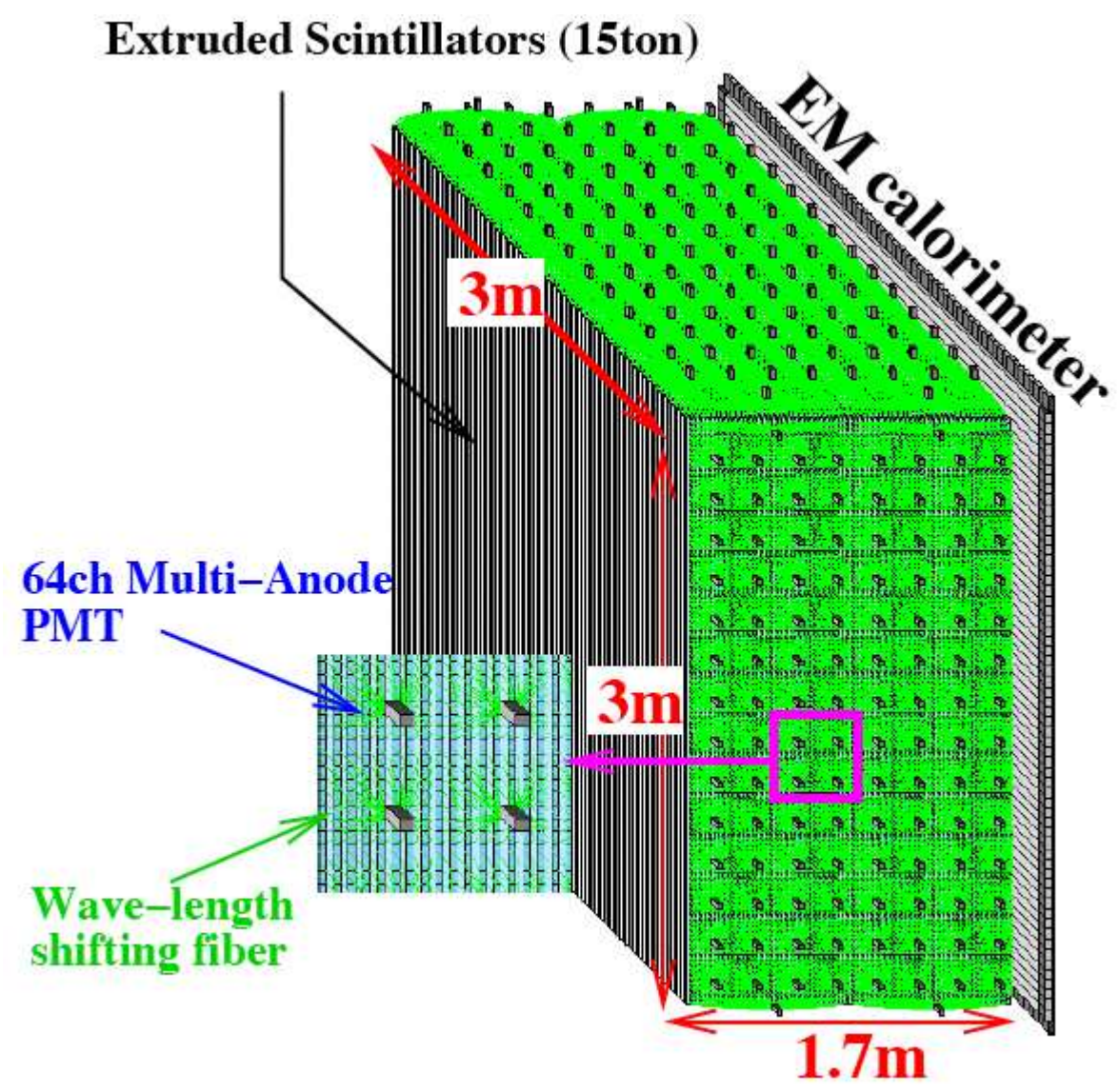

Figure 3.5: The SciBar and Electron Calorimeter (EC) detectors. Each scintillator strip (white) is instrumented with a green wavelength shifting fiber in the center, which make up the SciBar detector. An inset shows how groups of 64 fibers are read into a MA-PMT (grey rectangle). The EC is a two-plane calorimeter made up of lead foil and scintillating fibers attached to the downstream end of SciBar. The neutrino beam is incident from the left on the figure. 
in place by a plastic alignment "cookie" which interfaces to a 64-channel multi-anode PMT (MA-PMT, Hamamatsu H8804). The average gain for 64 channels is $6 \times 10^{5}$. The cross talk (light from one fiber affecting a nearby channel on the PMT) was measured to be $3.15 \pm 0.4 \%$ for an adjacent channel, and $0.7 \%$ for a diagonal channel [35].

Charge and timing information is read out by a front end board (FEB) attached to the MA-PMT. Data acquisition boards (DAQ) read out 8 FEBs with a typical pedestal width less than 0.3 photo electrons (pe).

A special calibration system is built into SciBar [35]. A single clear fiber sits in the center of each bundle which injects light from an blue LED matched to the WLS absorption spectrum. The response to the LED light provides a measurement of the gain of the MAPMTs over time as well as indicating which channels are dead.

SciBar was first assembled as a near detector for the K2K experiment in January 2003 and ran successfully $[36,16,37]$ until the end of the K2K run in November 2004. The advantage of reusing a detector is the ability to better understand it and improve it. For example, for the K2K run of SciBar, the interface between the PMT and the cookie had optical grease to increase light output, however, this increased also the crosstalk between channels. For the SciBooNE run, the optical grease was removed.

SciBar was shipped from Japan and re-assembled at Fermilab. After testing with cosmic rays, the detector was put on a truck and lifted into the SciBooNE detector hall. The detector hall was designed to have a removable roof, as the detector hall itself was kept as small as possible. Over the entire SciBooNE run, only 4 channels out of 14,336 failed permanently.

\subsubsection{The Electron Catcher (EC)}

Just downstream of SciBar is the EC, a "spaghetti" type calorimeter. The EC consists of two planes of 32 modules of $1 \mathrm{~mm}$ scintillating fibers in lead foil. Each module $(262 \mathrm{~cm} \times$ $8 \mathrm{~cm} \times 4 \mathrm{~cm}$ ) is read out by two 1" Hamamatsu PMTs, requiring 256 channels in total. There is one vertical plane and one horizontal plane, covering an active area of $2.7 \times 2.6 \mathrm{~m}^{2}$, as shown in Fig. 3.5. Only charge information is read out. The EC is 11 radiation lengths in the beam direction $(\sim 0.35$ interaction lengths). 
The EC was built for the CHORUS experiment [38] and later used in conjunction with SciBar for the K2K experiment. Additional details on the EC can be found under Ref. [35].

\subsubsection{The Muon Range Detector (MRD)}

The MRD consists of 12 iron plates interspersed with 13 layers of scintillator counters weighing 48 tons, as shown in Fig. 3.6. The iron plates, recycled from Fermilab experiment E602, cover an area of $274 \mathrm{~cm} \times 305 \mathrm{~cm}$, are $\sim 5 \mathrm{~cm}$ thick, with the average density for a spare plane measured to be $7.841 \pm 0.002 \mathrm{~g} / \mathrm{cm}^{3}$ measured at various points across the plate. The thickness of each individual plane was measured to 1\%. There are 6 horizontal layers with $2 \times 12$ counters and 5 vertical layers with $2 \times 15$ counters, for a total of 362 scintillator counters. Each counter is strapped to an aluminmum frame which is attached to an iron plate. Each individual counter is $138 \mathrm{~cm}$ long for vertical planes, $155 \mathrm{~cm}$ for horizontal, and both types are $6 \mathrm{~mm}$ thick and $20 \mathrm{~cm}$ wide. One end of each counter is glued to a plastic waveguide and optically cemented to a 2" PMT . Foil covers the lightguide and cookie, and each counter is wrapped in black Tyvec. Each PMT is additionally secured inside a cylindrical tube to reduce light leaks. As the MRD was assembled from spare parts around Fermilab, tubes from the NuTeV experiment (Hamamatsu R2154, operating voltage $\sim-1100 V$ ), KTeV experiment (EMI9954KB,EMI9839B and EMI9939B, $\sim \pm 2000 V$ ), and RCA6342A (10 stage, $\sim-1400 \mathrm{~V}$ ) were used. The 8th plane of the MRD has positive HV (EMI9939B) tubes. For the first antineutrino run, the positive HV bases were unmodified, so ADC information is absent for this plane. For subsequent runs, the bases were modified with the addition of a capacitor in the base in order to use ADC information.

531 tubes were tested for noise levels and operating voltage, with the best tubes used in the MRD and any acceptable tubes made into spares. Each counter was individually tested for light leaks, functional glue joints and proper grounding. Finally, before commissioning, the detector was fully cabled and run with cosmic ray data.

Like SciBar and the EC, the MRD was not assembled in the SciBooNE detector hall, as the detector hall was being built during counter testing and assembly. On April 23rd, 2007, the same day as the SciBar move, the MRD was moved from assembly and lifted by crane into the SciBooNE detector hall, as shown in Fig. 3.7. 


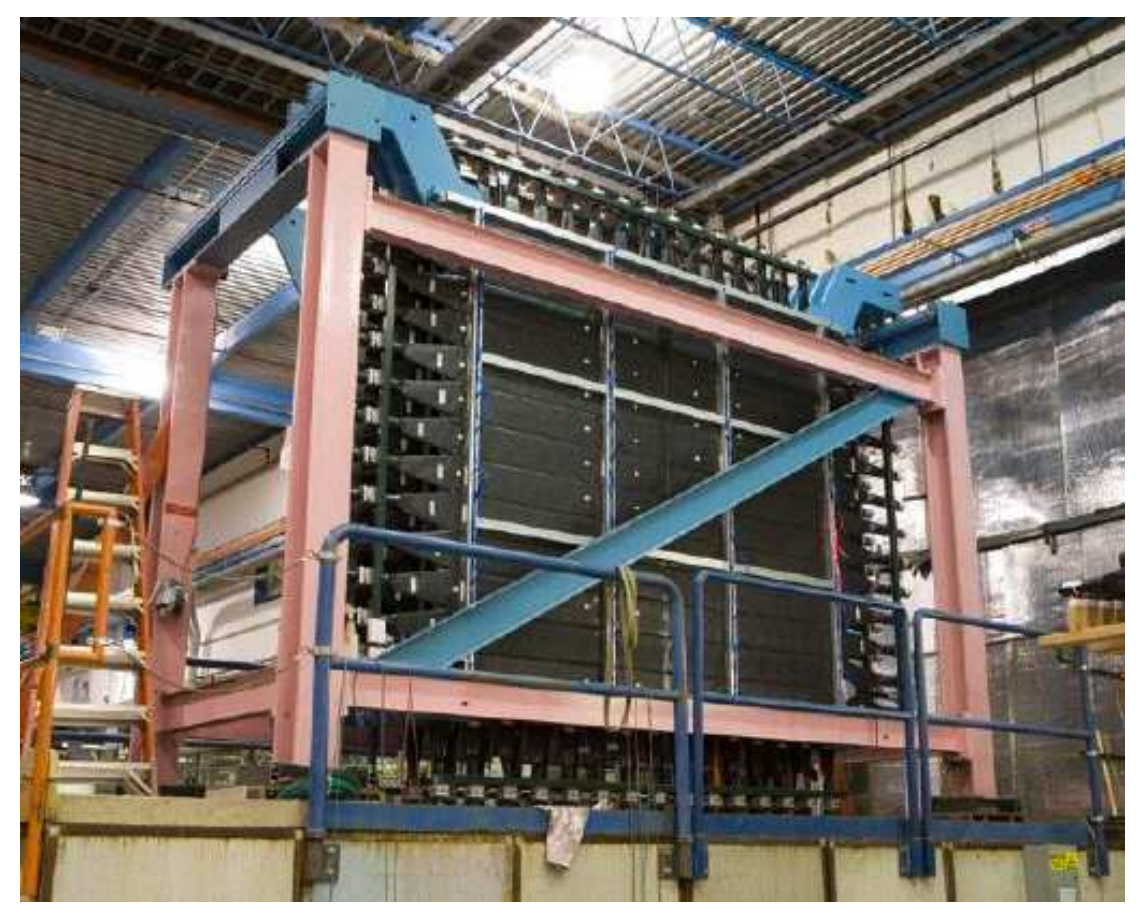

Figure 3.6: The MRD at the assembly point in Lab F of Fermilab. Black, light tight, scintillator counters are arranged in pairs in alternating horizontal and vertical planes, and are strapped to a (silver) aluminum frame with (blue) nylon. The PMTs are read out on the top, bottom and sides of detector. The (blue) iron planes sit on a (pink) support frame. 


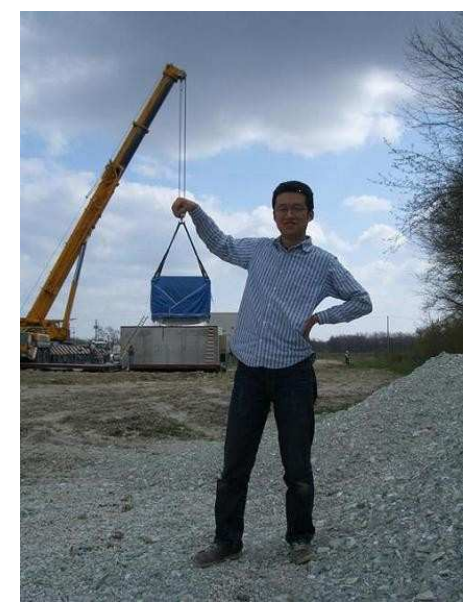

Figure 3.7: The 50 ton-rated crane lifts the MRD through the top of the SciBooNE hall. My fellow graduate student, Yasuhiro Nakajima, assists.

For the first run of SciBooNE, only 4 of the 362 PMTs broke due to transportation of the detector, and 2 tubes had low gain. During the shutdown between the first and second run, all the faulty PMTs were replaced. No further PMTs failed over the neutrino and second antineutrino runs.

SciBooNE has two kinds of triggers: beam and calibration. The beam trigger comes from the accelerator clock and does not depend on any activity in any of the three detectors. During any non-beam trigger time, three calibration triggers can occur: pedestal, LED (SciBar only) and cosmic ray data. The pedestal and LED data is collected once between beam intervals. The cosmic trigger can occur at maximum 20 times between beam intervals, and is read out separately any time there is activity in either SciBar (and EC) and the MRD.

Calibration triggers provide important information about the detector response to muons. The LED system measured the gain of SciBar over the entire run to be stable to $\pm 2 \%$. A problem with particular readout electronic cards caused groups of channels to not respond to the LED light for $1.5 \%$ of the total SciBooNE run.

Cosmic ray triggers allow for direct measurement of minimum ionizing particles (MIPs) in all three detectors. A MIP deposits energy, giving 20 pe over $1.3 \mathrm{~cm}$ distance traveled in the SciBar detector, with a timing resolution of $1.6 \mathrm{~ns}$. A MIP deposits $91 \mathrm{MeV}$ as it passes 
through the EC. A MIP of energy greater than $\sim 1.2 \mathrm{GeV}$ exits the MRD. The average hit finding efficiency for the MRD is $99 \%$, calculated using the cosmic ray trigger events.

SciBooNE is a tracking detector. When a particle passes through an extruded scintillator strip, it produces scintillation light. The light travels down the WLS fiber to the MA-PMT, and as the particle moves, subsequent strips are hit in $\mathrm{x}$ and $\mathrm{y}$ planes. The time and charge of the light can be reconstructed to form tracks which indicate the position, angle and energy of the particle. The EC is a calorimeter, so it acts more like a binary switch. Electrons are stopped in the EC, and deposit all their energy, but muons pass through to the MRD. Hits in the EC just produce charge information with lesser position information, as there are just two planes. The counters in the MRD are scintillator as well, and function in much the same way as strips in SciBar do, albeit with cruder position information as the counters are larger.

A typical neutrino event in SciBooNE is shown in Figure 3.8. The muon is the longer track which passes through most of SciBar, the EC and stops in the MRD. The proton track is reconstructed, and is the shorter second track which stops in SciBar.

\subsection{The MiniBooNE Detector}

MiniBooNE is a spherical, 950,000 liter mineral oil Cherenkov detector [39], as shown in Fig. 3.9. MiniBooNE uses 1280 (240) 8" Hamamatsu PMTs in the inner (outer) region. 1198 of the PMTs are R1408 (9 stage) recycled from LSND, and 322 are R5912 (10 stage) bought for MiniBooNE. The tubes are oriented as shown in Fig. 3.10, where inner PMTs face inward, while in the outer, optically isolated "veto" region, PMTs are mounted back to back facing up or downwards. The PMTs are arranged in horizontal layers on opaque panels, which, when combined with latitudinal hoops, allow oil to flow between outer and inner regions but prevent light leakage. All surfaces in the veto region are painted white to increase light re-scattering, and in the inner region are covered or painted black in the to reduce re-scattering. The exception to this is the metal support structure, which remains unpainted to minimize oil interactions with the paint over time.

The MiniBooNE detection medium is pure Marcol 7 mineral oil. Mineral oil has a 


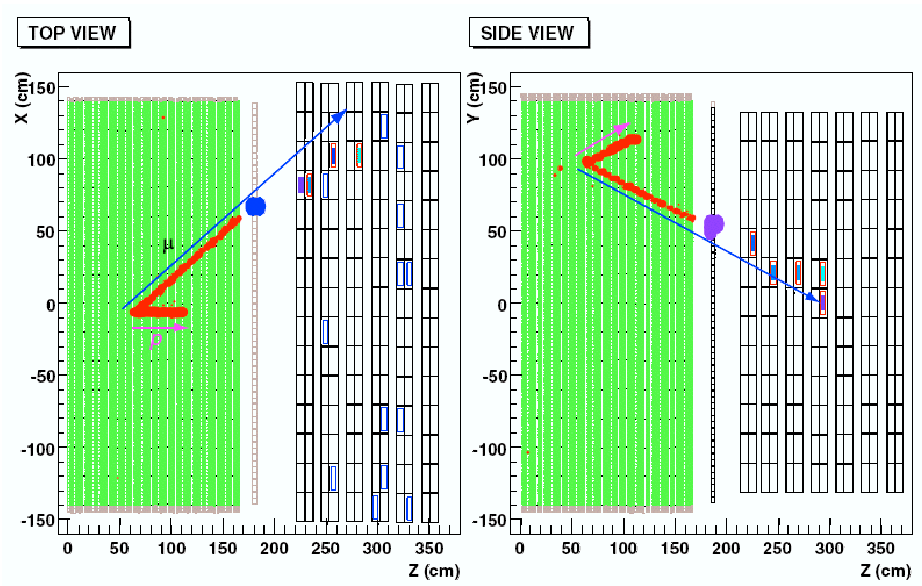

Figure 3.8: A typical neutrino event in SciBooNE. The red circles denote ADC hits in SciBar, with the size of the circle indicating charge deposited. Blue and purple circles indicate charge and hits in the EC. Open boxes indicate hits in a MRD counter; beam on (off) hits are in red (blue). Closed boxes indicate charge information for the beam on hits. Left: The "top view" of the detector, shown as if the viewer was looking down on the detector, with x position and the beam direction $(\mathrm{z})$ in $\mathrm{cm}$. Right: The "side view" of the detector, shown as if the viewer was looking at the detector from the side, with y position and the beam direction $(\mathrm{z})$ in $\mathrm{cm}$. 

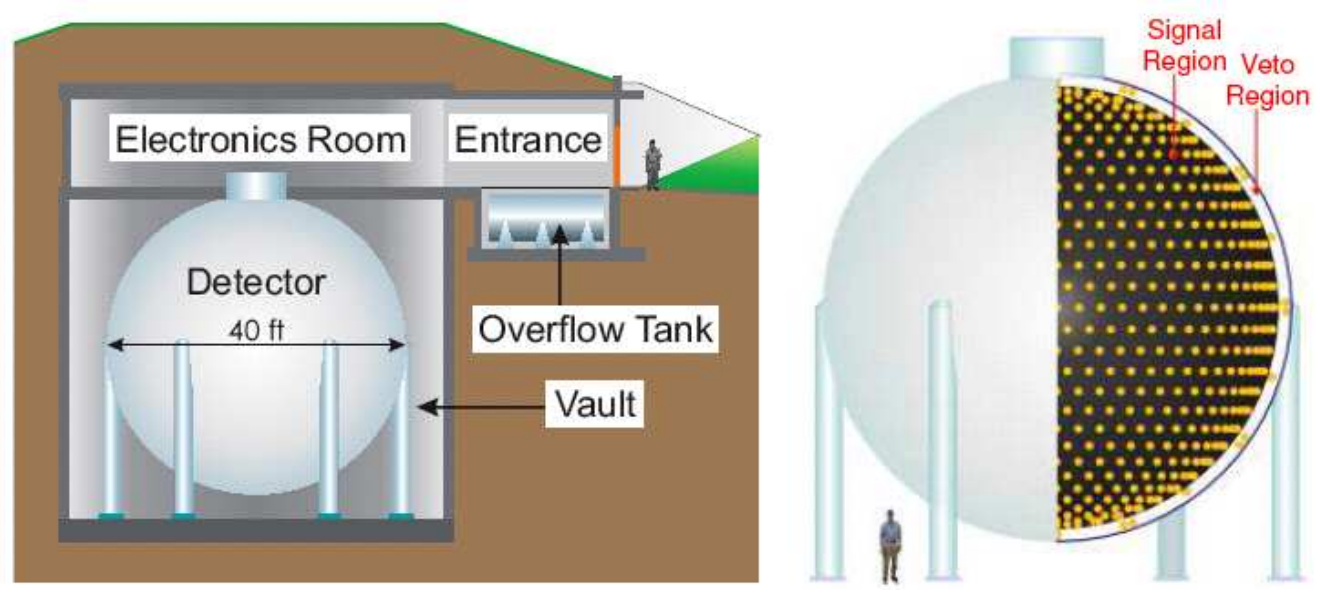

Figure 3.9: Left: The MiniBooNE detector in the detector hall. Right: The signal region of the detector has phototubes are arranged in vertical rings around the inside of the spherical tank, filled with mineral oil. The outside of the tank is an optically isolated "veto" region.

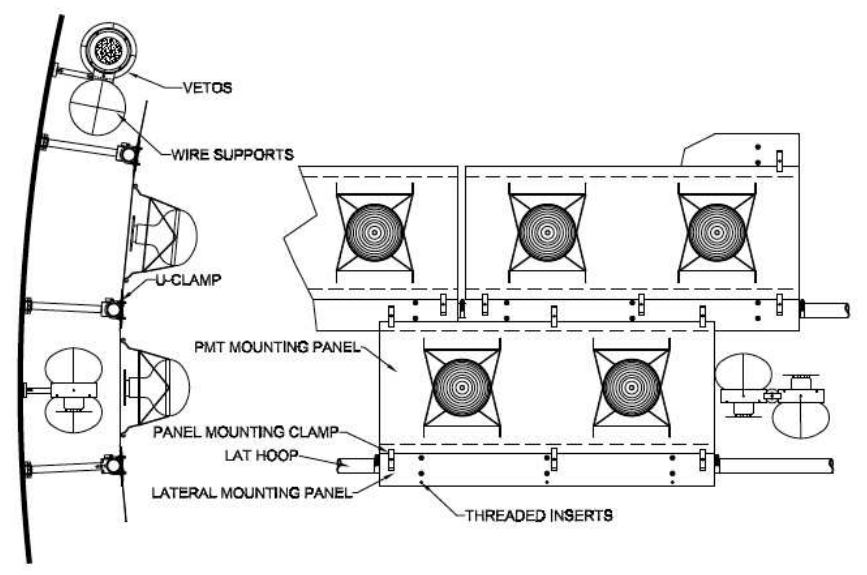

Figure 3.10: The inner PMTs face inward, and are arranged on latitudinal rings, staggered with respect to rows above and below. The outer PMTs are mounted facing upwards or downwards in pairs. 
lower density than water $\left(\eta=0.845 \pm 0.001 \mathrm{~g} / \mathrm{cm}^{3}\right) ;$ a $2 \mathrm{GeV}$ muon travels approximately the full length of the $12 \mathrm{~m}$ diameter tank. The oil is kept in a nitrogen environment, as oxygen in the oil can cause the attenuation length to decrease, and can cause oxidation on any exposed metal. The mineral oil was bubbled with $N_{2}$ after the filling of the tank to remove excess oxygen; currently, the oil is not recirculated.

The PMTs are operated at $+\sim 2000 \mathrm{~V}$, which gives a gain of $\sim 1.6 \times 10^{7}$. The intrinsic time resolution on the PMTs is $\sim 1 \mathrm{~ns}$, and the intrinsic charge resolution is $\sim 15 \%$ at 1 pe. During the three month comissioning 24 PMTs failed, and as of March 6th, 200913 additional tubes have failed (37 total).

Charge $(q)$ and timing $(t)$ information are read out when the charge on a PMT is greater than $2 \mathrm{mV}$ ( $\sim 0.1$ pe), called a "hit". A given PMT cannot be read out again for 200-300 ns, depending on the time of the initial hit. The ADC digitizes and stores information $(q, t)$ for $200 \mu s$ to be read out whenever there is an external signal for data taking, a "trigger". The main relevant triggers for the analysis in this thesis are the beam and strobe triggers. The beam trigger comes from the accelerator clock and does not depend on PMT activity in the tank, and opens a time window $5 \mu \mathrm{s}$ before the $1.6 \mu \mathrm{s}$ beam spill, and holds off acceptance of subsequent triggers for the full $19.2 \mu \mathrm{s}$ readout window. The strobe trigger is a $2.01 \mathrm{~Hz}$ pulser which provides an unbiased sample of beam-off events, such as cosmic ray events.

MiniBooNE has a "shallow" overburden of at minimum $3 \mathrm{~m}$ of dirt, so approximately $10 \mathrm{kHz}$ of cosmic ray events reach the tank. Based on the analysis of strobe triggers, $99.987 \%$ of cosmic ray events above 200 main tank hits are rejected by imposing a cut of more than 6 hits in the veto region.

As MiniBooNE is a Cherenkov detector, when a particle enters the tank or is produced in the tank, it can be traveling faster than the speed of light in mineral oil $(v=c / n)$ where $n=1.6$ is the index of refraction in mineral oil. When this happens, the particle will produce Cherenkov radiation, prompt light emitted at a characteristic angle with respect to the particle's direction, $\cos (\theta)=\frac{c}{n v}$. As the particle travels, it produces a cone of light in the forward direction.

The Cherenkov light is detected by the PMTs which line the inside of the tank. An 


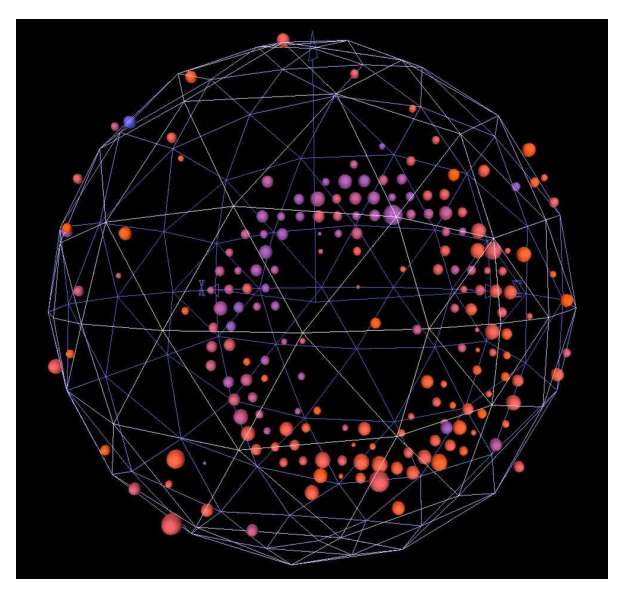

Figure 3.11: A typical muon event in MiniBooNE. The white frame represents the inner tank surface. Color indicates time (red $\rightarrow$ blue is early to late time, and size of the spheres represents the amount of charge deposited.

example of a muon in MiniBooNE is shown in Fig. 3.11. The conical Cherenkov light will show up as a series of "rings" on the inside of a spherical tank; the charge measured by the PMTs corresponds to the charge of the particle.

Incoming cosmic ray muons provide a natural calibration source for understanding muons on the detector. A two-plane scintillator tracker sits above the tank and provides directional information on muons entering the tank, as shown in Fig. 3.12. The angular resolution of the tracker is $\sim 1.9^{\circ}$. Deployed throughout the tank are six sealed scintillator cubes read out by an optical fiber leading to a 1" PMT. When a muon decays in a cube, the resulting decay electron will provide light in the scintillation cube. The combination of the tracker and the scintillation cubes provide the trajectory of the muon and the distance traveled in the tank. As muons are minimum ionizing particles, the energy of muons in the mineral oil can be calibrated from the $\sim 100$ events per month which stop in the cubes. Data from the muon tracker and cubes are compared to the result of the muon track fitter in Fig. 3.13, which shows linearity as well as consistency between prediction and data. Fig. 3.14 shows, for a particular range in muon energy, the angular resolution $\left(\sim 4^{o}\right)$ for the fitter using tracker data. The specifics of the muon fitter are discussed in more detail under Section 5.2.2. 

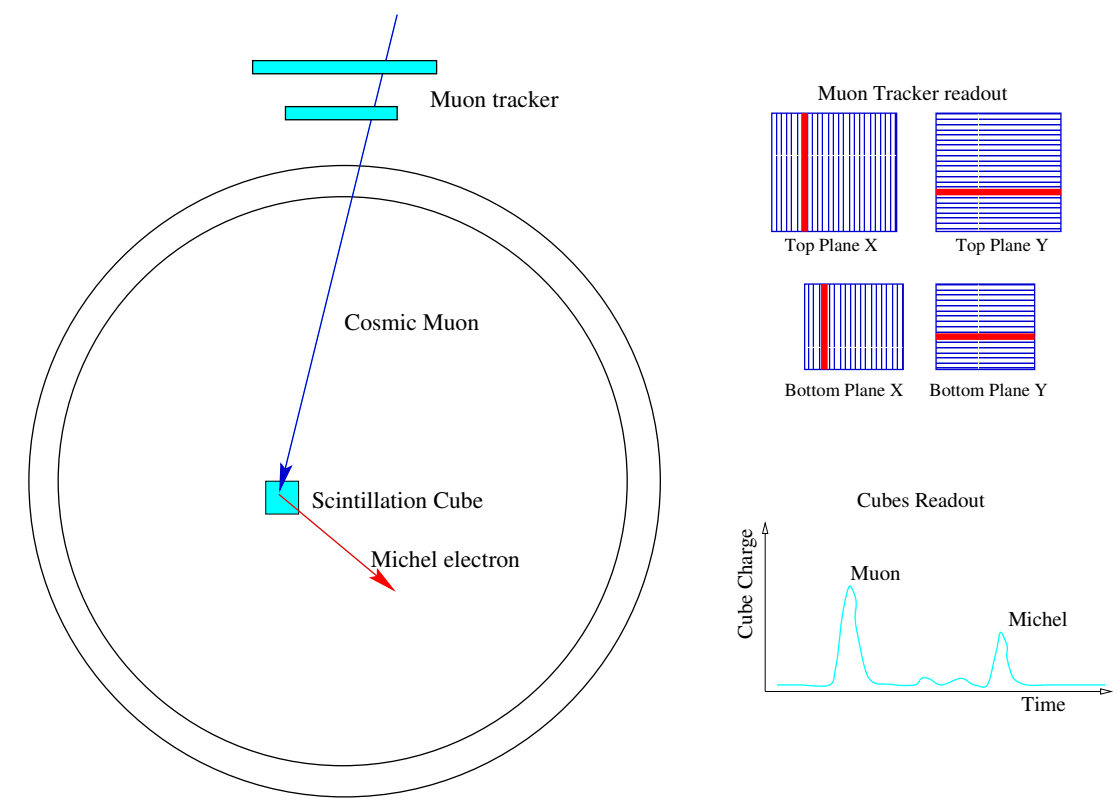

Figure 3.12: A schematic of the muon tracker and scintillation cubes. Two planes each with an $\mathrm{x}$ and $\mathrm{y}$ ( $\mathrm{z}$ is vertical) sit on top of the MiniBooNE detector. Muons enter through the tracker and stop in scintillation cubes (one shown, six are deployed throughout the tank). 


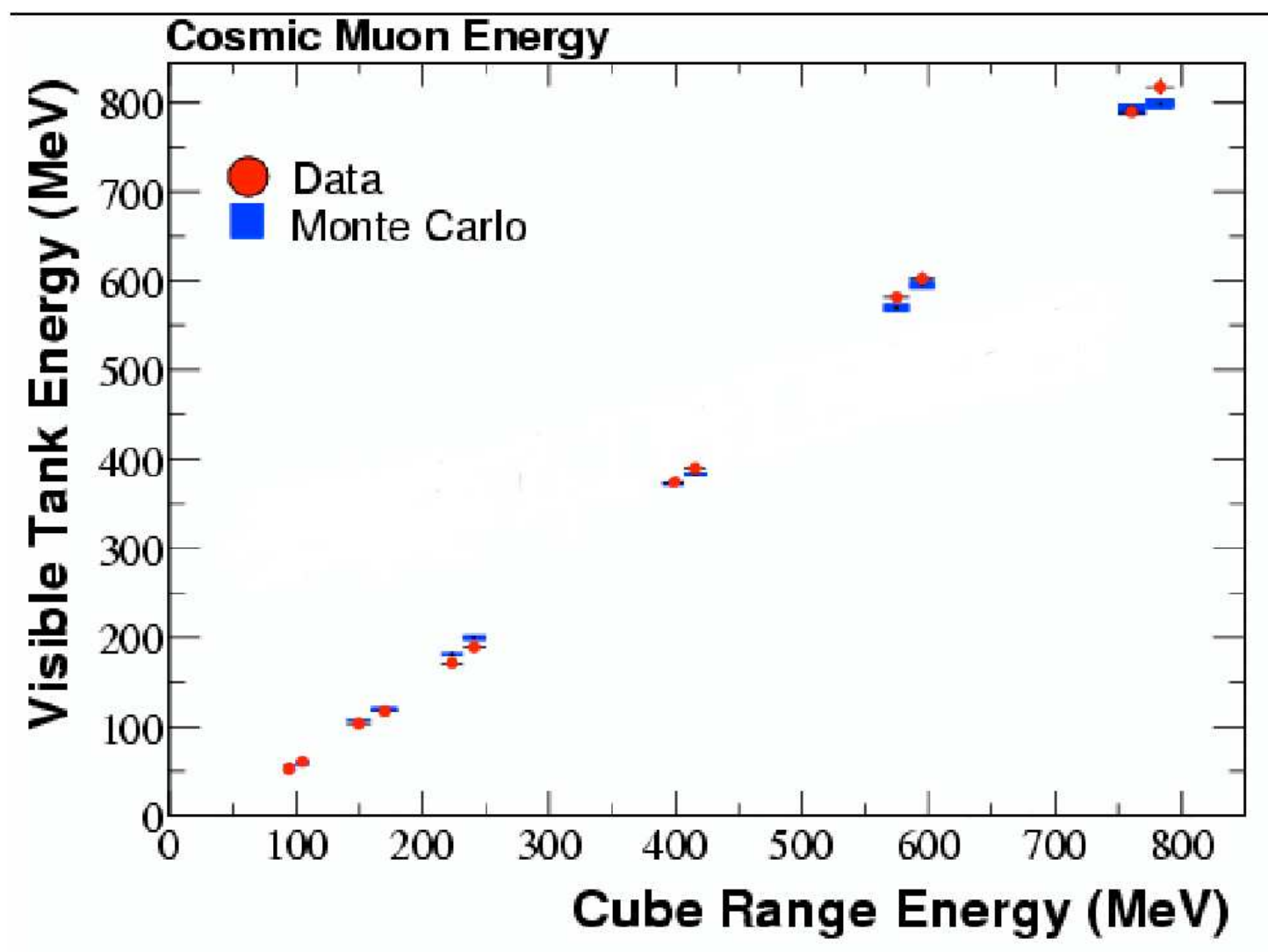

Figure 3.13: Reconstructed energy using a fit versus reconstructed energy based on distance using the muon tracker. The reconstructed energy from the cube is calculated between the tank entry point from the muon tracker plane information and the center of the corresponding scintillation cube and by applying the formula for MIPs in mineral oil. The non-zero intercept is due to the visible energy not including the muon mass (electronequivalent energy). The reconstructed energy is the output of the standard reconstruction fitter used for the MiniBooNE $\nu_{\mu}$ analysis. Blue rectangles are the prediction (error bars are the size of the rectangle), compared to the cosmic ray muon data (red circles) with two bins for each cube. 


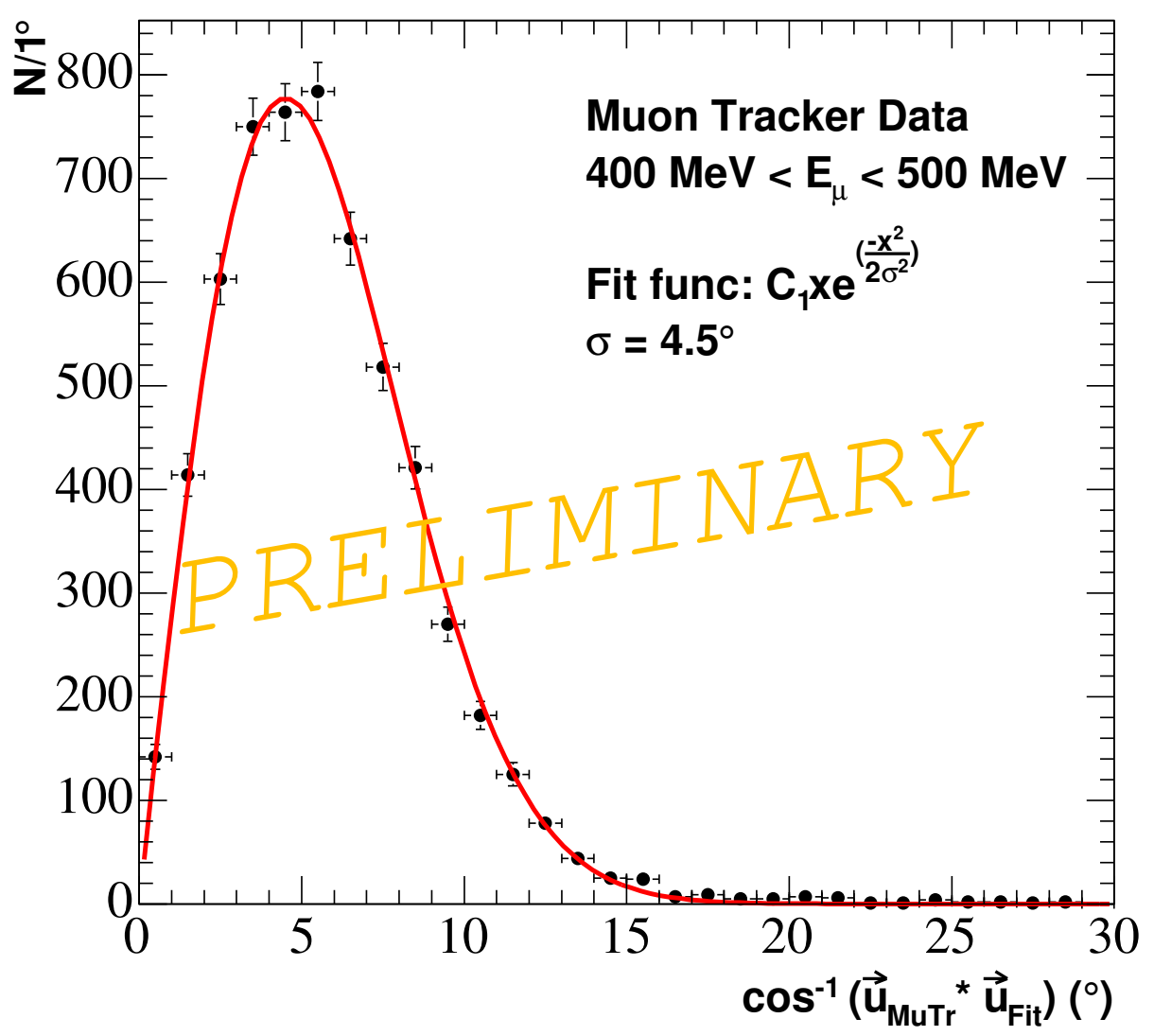

Figure 3.14: Angular resolution, using the standard muon fitter, for muon tracker events with $400<E_{\mu}<500 \mathrm{MeV}$. The spread is calculated to be $4.5^{\circ}$ from a symmetric 2D Gaussian projected onto the radial direction (1D) 
In addition, mineral oil scintillates when charged particles pass through it. Scintillation light is isotropic, and is caused by photons being absorbed and remitted, so scintilla-

tion light is delayed and the amount produced is proportional to $e^{\frac{-t}{\tau}}$, where $\tau$ is the time constant of the decay.

A given material may have many scintillation components, and this is true for mineral oil. A host of external measurements of the oil properties were made, including: scintillation light using a proton beam, fluorescence spectroscopy, time-resolved spectroscopy, and attenuation length, as shown in Fig. 3.15. The interplay between scintillation light and Cherenkov light is shown for a muons in Fig. 3.16; the dominant component of light for muons in MiniBooNE is the Cherenkov light.

Additional details about the MiniBooNE detector can be found under Ref. [39].

\subsection{Run Periods}

MiniBooNE took neutrino data corresponding to $5.579 \times 10^{20}$ POT after the data quality cuts (see Section 5.2.3) are applied. MiniBooNE also ran with a neutrino beam for two more distinct sections of time, as summarized in Table 3.1, which shows run number and POT (Toroid_860, Toroid_875 gives a value of $5.552 \times 10^{20}$ POT). While the additional $0.882 \times 10^{20}$ POT is used for the joint MiniBooNE/SciBooNE analysis, the addition of the extra data has a negligible effect on the MiniBooNE-only analysis and so, for direct comparison to the cross section paper, the $5.579 \times 10^{20}$ POT data set is used for the neutrino disappearance fit. The total antineutrino data taken corresponds to $3.386 \times 10^{20}$ POT after data quality cuts.

The stability of neutrino events over time is shown in Fig. 3.17; over two and a half years the neutrino rate has been consistent with no change within the $1 \%$ error on the POT. The corresponding plot for antineutrino running over two years is shown in Fig. 3.18; the two drops in the event rate are due to the first and second absorber plates discussed earlier.

SciBooNE started data taking on May 30th, 2007 while the beam was in antineutrino mode, and after the absorber plates were removed. After the joint antineutrino run, Sci- 


\section{Extinction Rate for MiniBooNE Marcol 7 Mineral Oil}

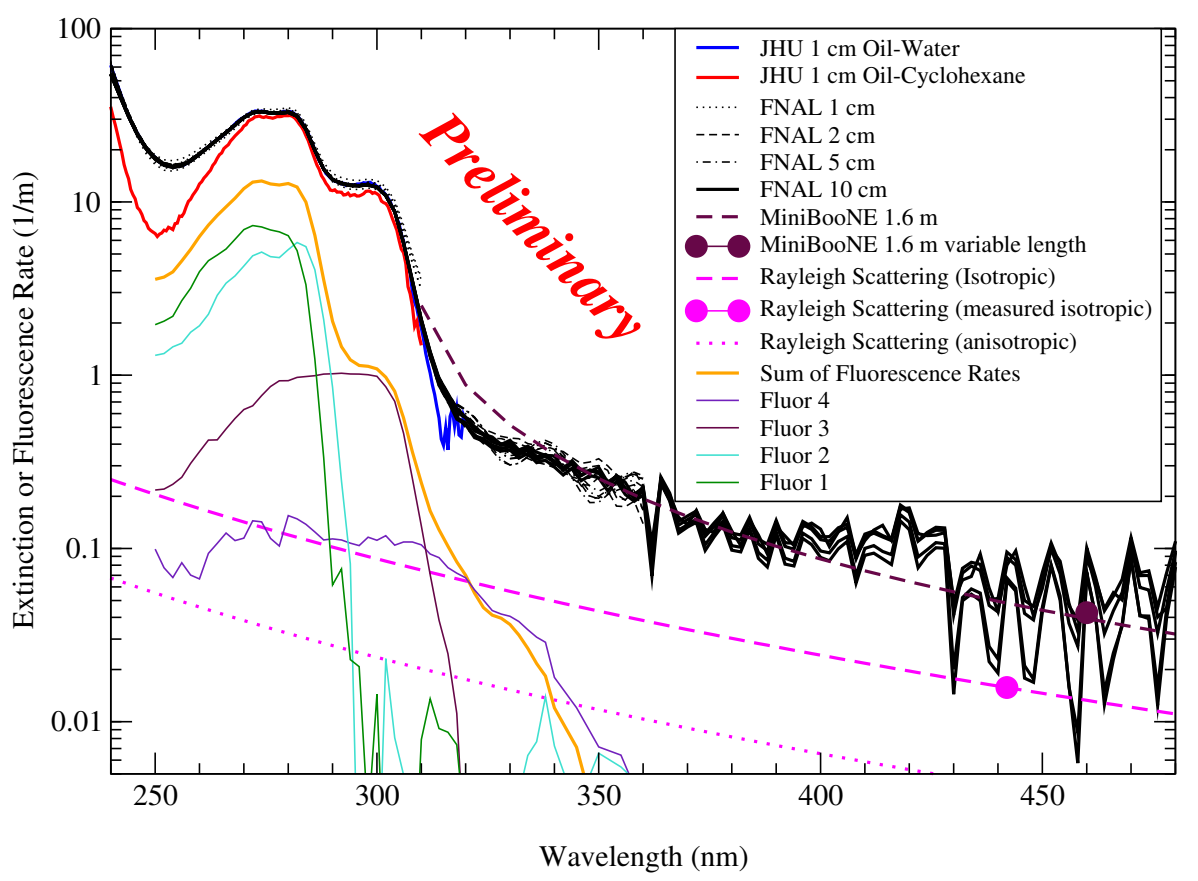

Figure 3.15: Extinction rate measurements of the MiniBooNE oil. At Johns Hopkins University (JHU), transmission compared with water (corrected for reflections) using a spectrophotometer with $1 \mathrm{~cm}$ cell are shown in blue. Measurements at JHU comparing Marcol 7 to cyclohexane are shown in red. Measurements with the spectrophotometer at Fermilab with cells of $1 \mathrm{~cm}(<310 \mathrm{~nm}), 2 \mathrm{~cm}(<360 \mathrm{~nm}), 5 \mathrm{~cm}(<360 \mathrm{~nm})$, and $10 \mathrm{~cm}$ are shown with black lines of various styles. The maroon dot shows the result from the MiniBooNE $460 \mathrm{~nm}$ variable path length instrument [40]; the dashed maroon line uses data from the MiniBooNE $1.6 \mathrm{~m}$ variable wavelength instrument [41]. The magenta dot shows the results of scattering measurements at Princeton $U$ [42] for the isotropic component of Rayleigh scattering. A matrix of emission and excitation fluorescence spectra measured in a spectrofluoremeter, yielding the components shown with solid green, turquoise, maroon and indigo lines [43]. 


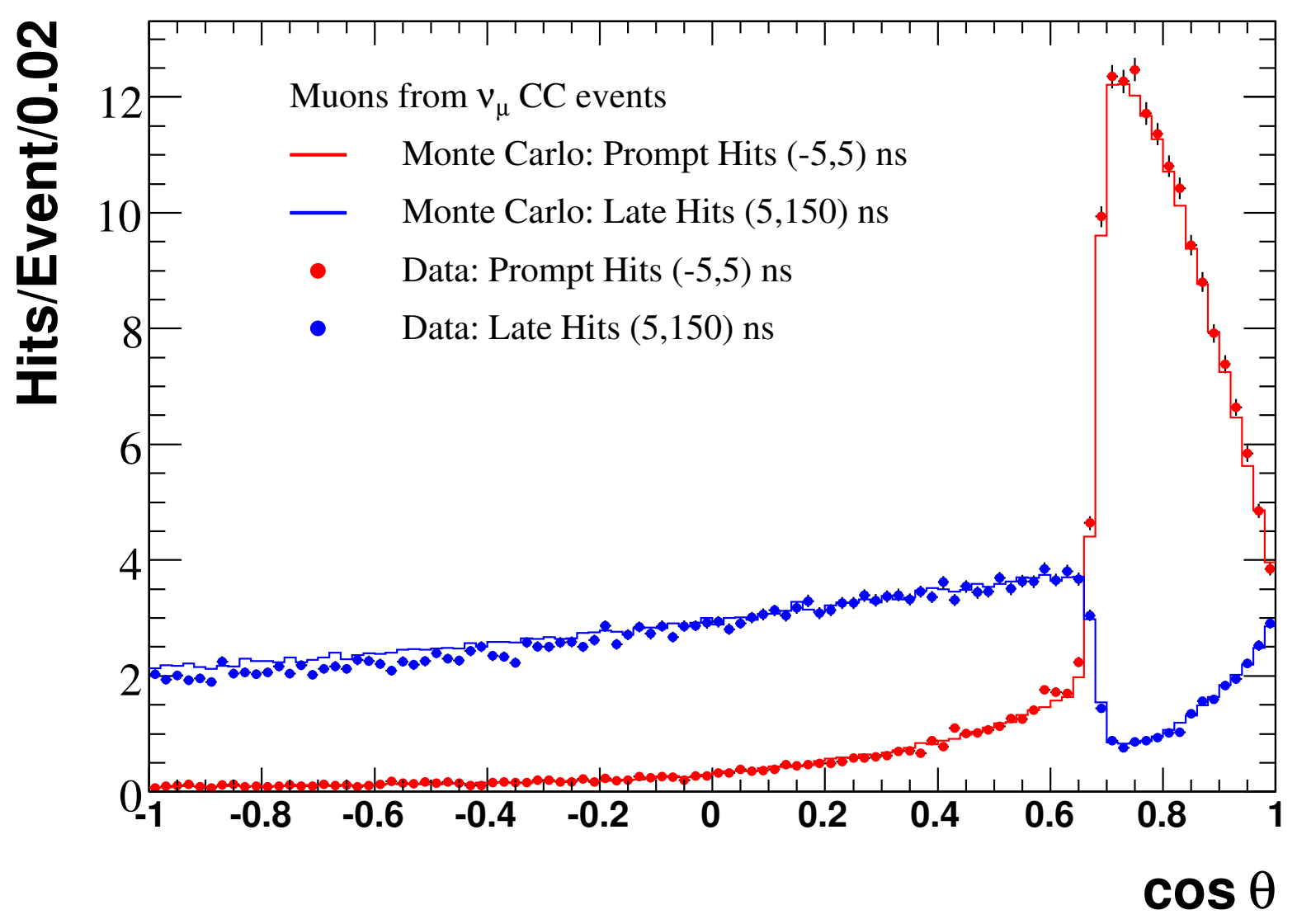

Figure 3.16: The angular profile of PMT hits relative to the reconstructed direction for muons with kinetic energy between 400 and $500 \mathrm{MeV}$. The red histogram (prediction) and points (data) shows the angular profile PMT hits with corrected time between -5 and $5 \mathrm{~ns}$. The blue histogram (prediction) and points (data) shows the angular profile for PMT hits with corrected time between 5-150 ns. The region near the Cherenkov peak is suppressed due to the dead time of the electronics; once a PMT registers a prompt hit, it cannot record later hits. Muon selection is similar to the CCQE $\nu_{\mu}$ selection discussed in Section 5.2.4. 


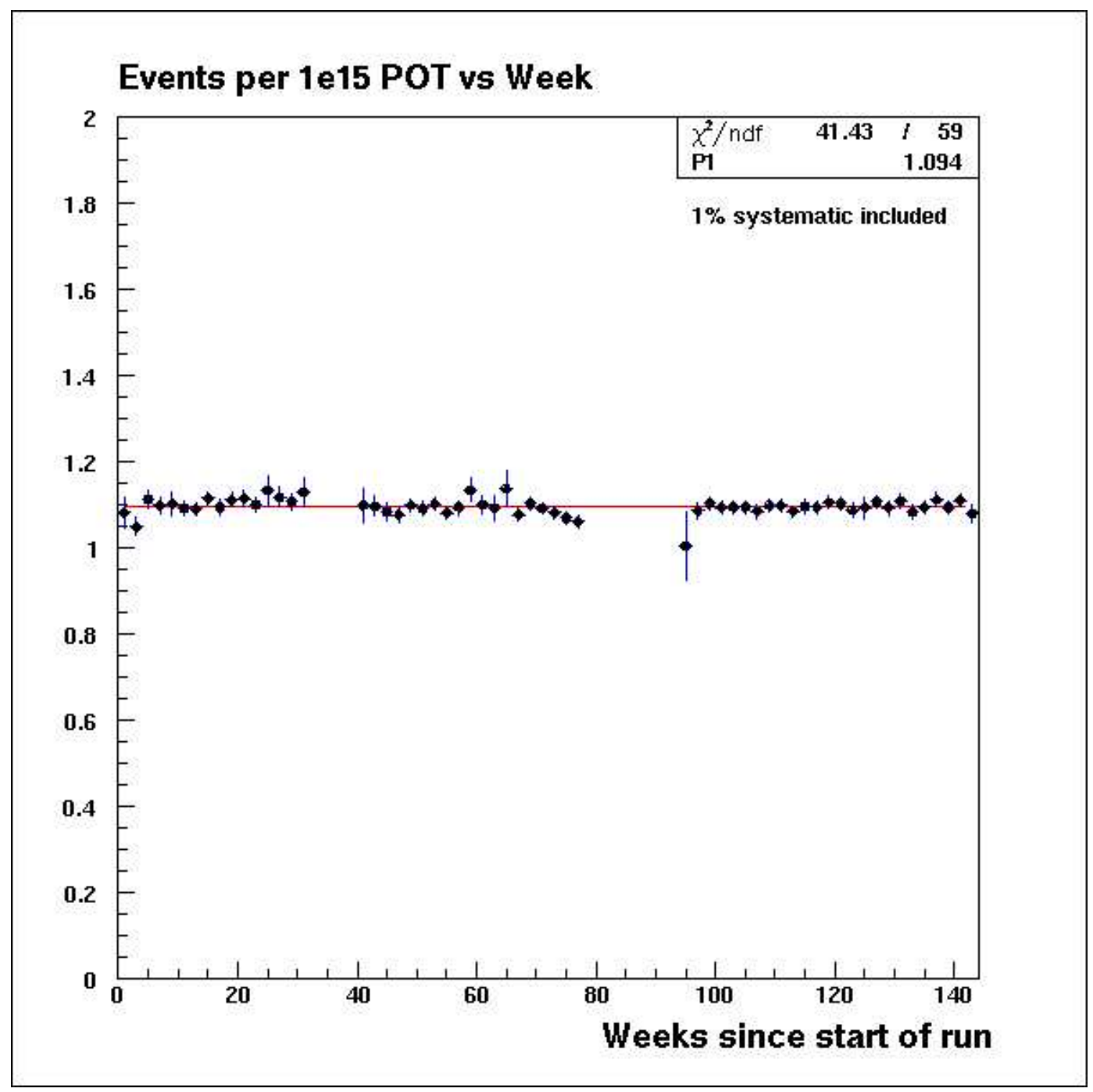

Figure 3.17: Neutrino candidates per POT vs number of weeks over the first neutrino run. The error bars include a $1 \%$ systematic error to account for variations in the calibration of the POT measurement. Neutrino candidates are defined in Section 5.2.1. 


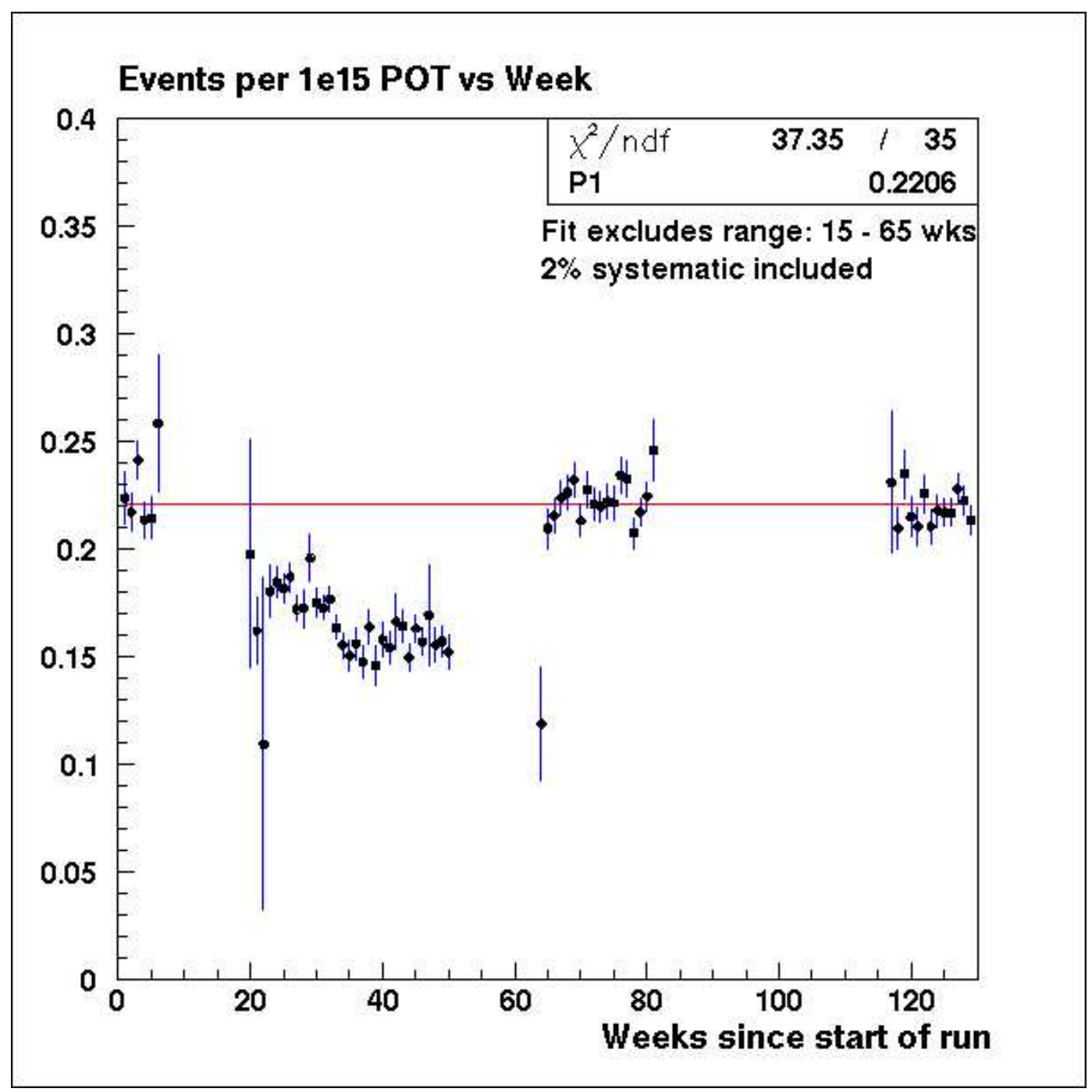

Figure 3.18: Antineutrino candidates per POT vs number of weeks over the first neutrino run. The error bars include a $1 \%$ systematic error to account for variations in the calibration of the POT measurement. Antineutrino candidates are defined in the same way as neutrino candidates (Section 5.2.1). 


\begin{tabular}{|c|c|c|c|}
\hline Name & MB run start & MB run end & POT $\left(\times 10^{20}\right)$ \\
\hline$\nu$ run Ia & 3539 & 12499 & 5.579 \\
$\nu$ run Ib & 12500 & 12842 & 0.050 \\
$\bar{\nu}$ run Ia (0 absorber) & 12843 & 14048 & 0.172 \\
$\bar{\nu}$ run Ib (1 absorber) & 14049 & 14362 & 0.569 \\
$\bar{\nu}$ run Ic (2 absorber) & 15119 & 15832 & 0.972 \\
$\nu$ run II & 15833 & 17160 & 0.832 \\
$\bar{\nu}$ run II (0 absorber) & 17161 & 18425 & 1.061 \\
$\bar{\nu}$ run III (0 absorber) & 18425 & $19000+$ & ongoing \\
\hline
\end{tabular}

Table 3.1: The distinct periods of neutrino and antineutrino running on MiniBooNE, chronologically. POT is given for Toroid_860 after data quality cuts and bad run cuts are applied. $\mathrm{X}$ absorber denotes the number of absorber plates in the beamline for that run period.

\begin{tabular}{|c|c|c|c|}
\hline Name & SB run start & SB run end & POT $\left(\times 10^{20}\right)$ \\
\hline $\bar{\nu}$ (run I) & 10000 & 10377 & 0.52 \\
$\nu$ (run II) & 11001 & 12132 & 0.99 \\
$\bar{\nu}$ (run III) & 13000 & 13709 & 1.01 \\
\hline
\end{tabular}

Table 3.2: The distinct periods of neutrino and antineutrino running on SciBooNE, chronologically. POT is given for Toroid_860 after data quality cuts.

BooNE ran in neutrino mode, and decommissioned in August 2008.

The difference between the SciBooNE and MiniBooNE neutrino runs collected POT is due to different livetimes between the detectors and a known inefficiency in the MiniBooNE reprocessing. The cooling system at the MiniBooNE detector caused electronics to overheat and shut themselves off, leading to a 6e18 POT difference for the same time period. The remainder of the difference is in the data reprocessing on MiniBooNE; approximately $5 \%$ of data events and POT are removed that are potentially recoverable. 


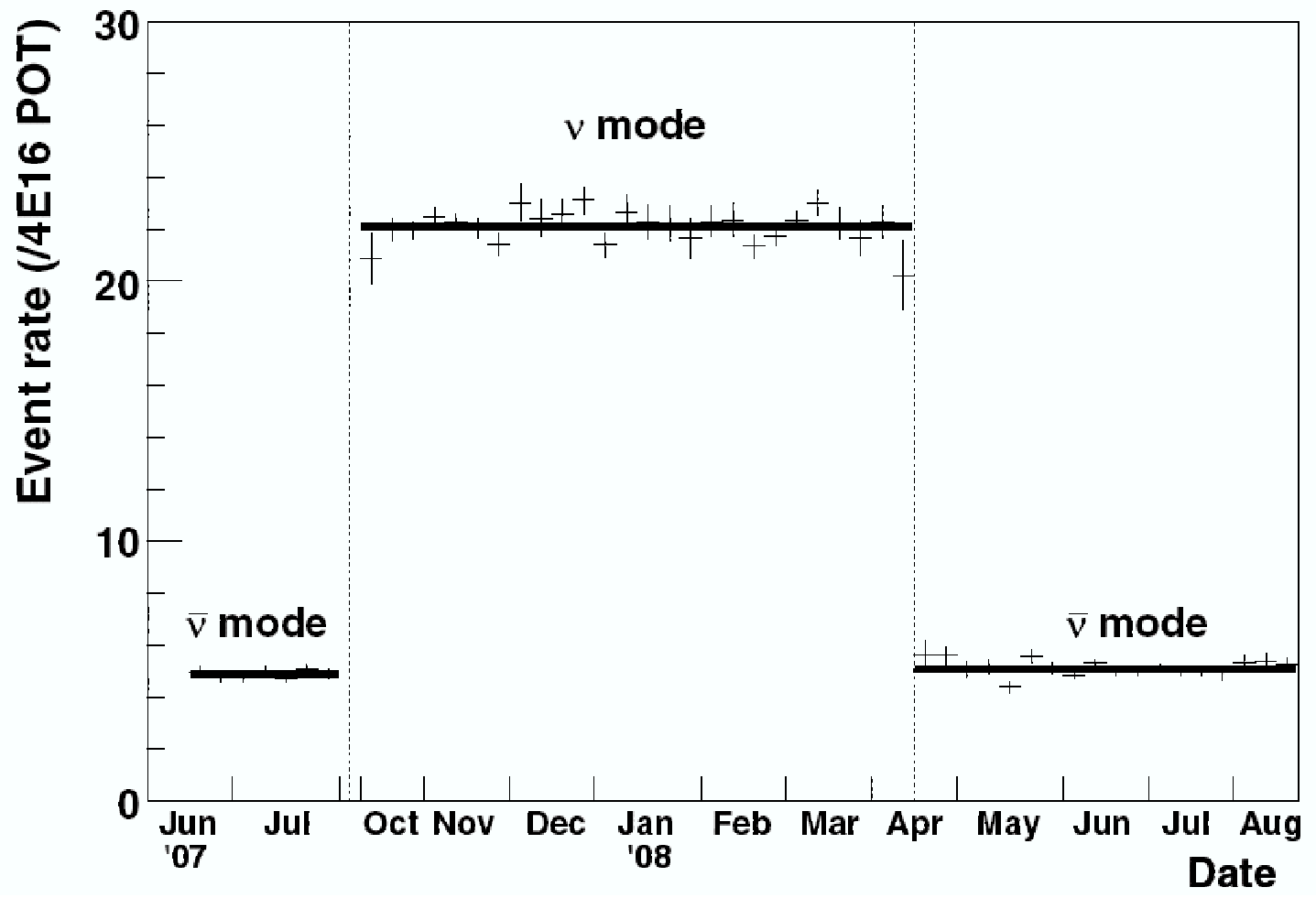

Figure 3.19: Neutrino and antineutrino candidates per POT vs date over the SciBooNE data taking run. Neutrino and antineutrino candidates are described under Section 5.1.3. 


\section{Chapter 4}

\section{Predicting the Neutrino Rate}

This section discusses how we simulate the production, interaction and detection of neutrinos from the BNB.

\subsection{Neutrino Source}

Both MiniBooNE and SciBooNE use the same GEANT4 based Monte Carlo simulation of the neutrino beamline. This section describes that simulation; more details can be found in Ref. [44].

The coordinate system convention used for both SciBooNE and MiniBooNE is a righthanded Cartesian coordinate system with the beam direction corresponding to $\mathrm{z}$, the vertical direction corresponding to $\mathrm{y}$.

The simulation can be separated into five main parts:

1. Geometry of the beamline

2. The initial proton beam

3. Proton/beryllium primary and secondary ( $\pi, K$ production) interactions

4. Magnetic field of the horn

5. Meson decay to neutrino beam

Each part is discussed stepwise, along with the assumed uncertainties. 


\subsubsection{Beamline Geometry}

The geometry is simulated as close to the real beamline as possible, including all materials used [44]. To change the neutrino flux by more than $1 \%$ would require changes in the position of the target or size of the beamline geometry by substantial amounts inconsistent with the accuracy of optical surveys. Therefore, no systematic uncertainty is attributed to the beamline geomtery.

\subsubsection{Proton Beam}

The incident proton beam is centered on the target, with a Gaussian spread in position of $\sigma_{x}=1.51 \mathrm{~mm}$ and $\sigma_{y}=0.73 \mathrm{~mm}$, based on beam monitors. An angular spread is also assumed corresponding to $\sigma_{\theta_{x}}=0.66 \mathrm{mrad}$ and $\sigma_{\theta_{y}}=0.40 \mathrm{mrad}$.

Variations of the incident proton beam have only small effects on the neutrino beam. Changing the focus point of the beam, the size of the transverse spread of the beam, and the angular spread (divergence) of the beam have less than 1\% effect on the neutrino beam.

\subsubsection{Proton-Beryllium Interactions}

The majority of protons interact in the Be target (interactions in other material use the default GEANT4 hadronic models). The total interaction cross section of primary proton interactions on Be is the sum of the elastic and the inelastic cross section. Inelastic collisions can be "quasi elastic" or "reaction" interactions where particles (e.g. $\pi, K$ ) can be produced.

Measurements exist for the total cross section on Be, as shown in Fig.4.1, and this is compared to the model, called the Glauber model, used in the simulation. For more information on the Glauber model, see Ref. [44]. The total interaction cross section is $\sigma_{\text {total }}=$ $285 \pm 15 \mathrm{mb}$ for protons at $8.9 \mathrm{GeV} / \mathrm{c}$. Inelastic cross section data is shown in Fig. 4.1 and is parameterized for use in the simulation. The inelastic cross section is $\sigma_{\text {inelastic }}=$ $212 \pm 5 \mathrm{mb}$. The elastic cross section is calculated using $\sigma_{\text {elastic }}=\sigma_{\text {total }}-\sigma_{\text {inelastic }}$. Both of these uncertainties have $\mathrm{a} \sim 1 \%$ effect on the neutrino flux. 

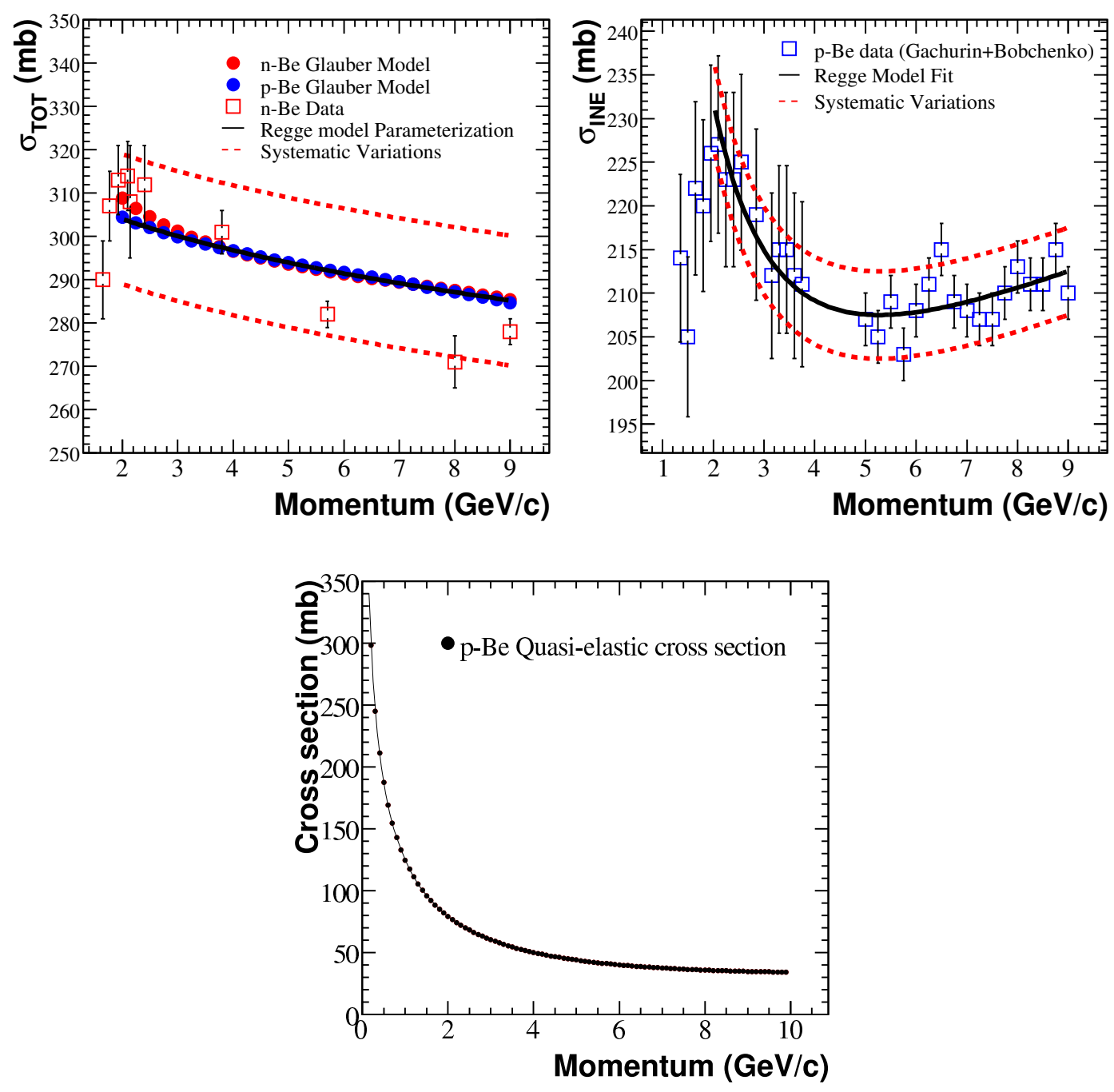

Figure 4.1: Top left: Total hadronic cross section for beryllium is shown for $n+B e$ interactions data (box) and prediction (red circle) and p+Be prediction (blue circle). The allowed systematic uncertainty is shown as well (red dash) Top right: Total inelastic hadronic cross section for beryllium is shown for $\mathrm{p}+$ Be data (blue box) and parameterization (black line). The allowed systematic uncertainty is shown as well (red dash) Bottom middle: Total quasi-elastic hadronic cross section for beryllium is shown for $\mathrm{p}+\mathrm{Be}$ and parameterization (black line). 
The quasi-elastic cross section has no available data in the region of interest, so a theoretical calculation, shown in Fig. 4.1 is used with a larger error ( $\pm 20 \mathrm{mb}$ for Be), which results in $\mathrm{a} \sim 2 \%$ change in the flux. Approximately $17 \%$ of all protons interact twice (scatter then produce a meson), this accounts for about $7 \%$ of the neutrino flux.

\subsection{4 $\pi$ and $K$ Production}

The main cross section of interest for a neutrino beam is how often a neutrino parent particle, typically a pion, is produced from $p+B e \rightarrow \pi^{+/-}+\ldots$. The differential pion production cross section has been measured by the HARP experiment [47], using the same beam energy $(8.9 \mathrm{GeV} / \mathrm{c} \mathrm{p})$ with a Be target $(2 \mathrm{~cm}$ thick in the beam direction). More information on HARP can be found under Ref. [48, 47]. Additionally, the E910 experiment measured the differential pion production at multiple beam energies $(6.4,12.3 \mathrm{GeV} / \mathrm{c})$. HARP and E910 data have a consistent normalization.

The differential cross section for pion production is described in the simulation with a Sanford-Wang (SW) parameterization [46]:

$$
\frac{d^{2} \sigma}{d p d \Omega}(p, \theta)=c_{1} p^{c_{2}}\left(1-\frac{p}{p_{B}-1}\right) \exp \left(-c_{3} \frac{p^{c_{4}}}{p_{B}^{c_{5}}}-c_{6} \theta\left(p-c_{7} p_{B} \cos ^{c_{8}} \theta\right)\right)
$$

where $\frac{d^{2} \sigma}{d p d \Omega}$ is the double differential cross section for pion production, $p$ is the momentum of the pion, $\theta$ is the angle of the pion with respect to the incident proton, $p_{B}$ is the momentum of the incident proton, and $c_{1}, . ., c_{8}$ are parameters.

While ideally, dedicated production data could be used directly to provide the cross section, a parameterization allows the simulation to interpolate between data points and extrapolate with the correct behavior at high and low momentum. To set the values of the parameters $c_{i}$, a $\chi^{2}$ is formed between the HARP and E910 data sets and the SW function and minimized:

$$
\chi^{2}=\sum_{k}\left[\sum_{i, j}\left(D_{i, k}-N_{k} T_{i}\right) \mathbf{V}_{i j, k}^{-1}\left(D_{j, k}-N_{k} T_{j}\right)+\frac{\left(N_{k}-1\right)^{2}}{\sigma_{k}^{2}}\right],
$$

where $D_{i, k}$ is the $i$-th data point for the $k$-th data set, $T_{i}$ is the value of the SW function for the kinematic parameters for that data point, $\mathbf{V}_{i j, k}$ is the bin-to-bin covariance matrix 


\begin{tabular}{l|rrrrrrrr}
\hline \hline & $c_{1}$ & $c_{2}$ & $c_{3}$ & $c_{4}$ & $c_{5}$ & $c_{6}$ & $c_{7}$ & $c_{8}$ \\
\hline$\pi^{+}$production & 220.7 & 1.080 & 1.000 & 1.978 & 1.32 & 5.572 & 0.0868 & 9.686 \\
\hline$\pi^{-}$production & 213.7 & 0.9379 & 5.454 & 1.210 & 1.284 & 4.781 & 0.07338 & 8.329 \\
\hline
\end{tabular}

Table 4.1: Extracted Sanford-Wang parameters $c_{1-8}$ for the $\pi^{+/-}$secondary production in p-Be interactions.

for the $k$-th data set, $N_{k}$ is relative normalization fit parameter for data set $k$ and $\sigma_{k}$ is the quoted normalization uncertainty for the data set $k$. The normalization uncertainty on HARP is $2 \%$, and on E910 is 5\%. The SW function is used to evaluate bin centering corrections, as the measured differential cross section is averaged over momentum and angle in each bin, but the cross section can vary across the bins. The fit is iterated with bin centering corrections until convergence is achieved. Parameter $c_{3}$ is strongly correlated to the other paramters, so for the $\pi^{+}$data fits, it was kept fixed at 1.0, and for the $\pi^{-}$fit was fixed to its best fit value.

The best fit $c_{i}$ values are given in Table 4.1 for $\pi^{+}$production and $\pi^{-}$production. Figure 4.2 shows the HARP data, the differential $\pi^{+}$cross section in angular bins as a function of pion momentum, as well as the SW parameterization.

The uncertainties on parent pion production are the largest of all the neutrino flux uncertainties. HARP measured the differential cross section to $4-7 \%$ across all momentum and angular bins; the uncertainty from HARP combined with the difference between the mean of the HARP data and the SW parameterization result in comparable 5-10\% uncertainties across neutrino energy. In regions where there is no HARP data, the errors spread accordingly. For details on how this is done in practice, see Section 6.3.1.

Pion interaction cross sections are handled in much the same way as proton interactions. Where data exists a parameterization is formed, otherwise input from theoretical calculations is used and checked against data scaled to Be. The total cross section for $\pi^{+/-}$is shown in Fig. 4.3, the inelastic cross section for $\pi^{+/-}$in Fig. 4.4 and the quasi elastic cross section is in Fig. 4.5.

The second major neutrino source in the BNB is from kaon decay. Though HARP took 

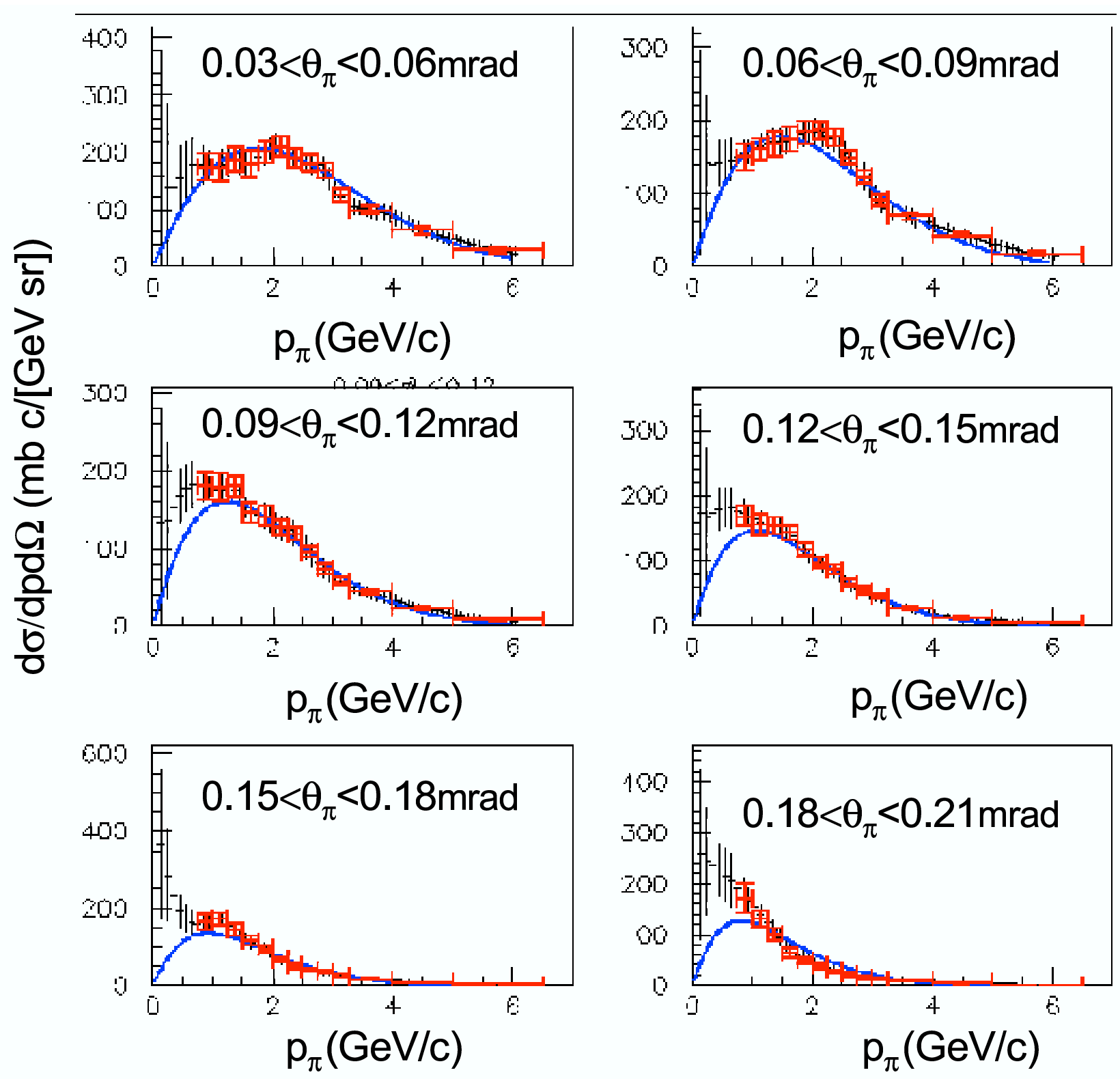

Figure 4.2: Comparison of $\pi^{+}$production cross section HARP data at $8.9 \mathrm{GeV} / \mathrm{c} \mathrm{p}$-Be (red, size of HARP errors shown by size of box) as a function of $p_{\pi}(\mathrm{GeV} / \mathrm{c})$, in bins of $\theta_{\pi}$, with the best fit SW model (blue line). Size of errors assumed on the $\pi^{+}$production derived from the HARP errors shown with black lines. Pions with momentum less than $1 \mathrm{GeV} / \mathrm{c}$ do not contribute to the $\nu_{\mu}$ flux in MiniBooNE or SciBooNE, as shown in Chapter 5. 

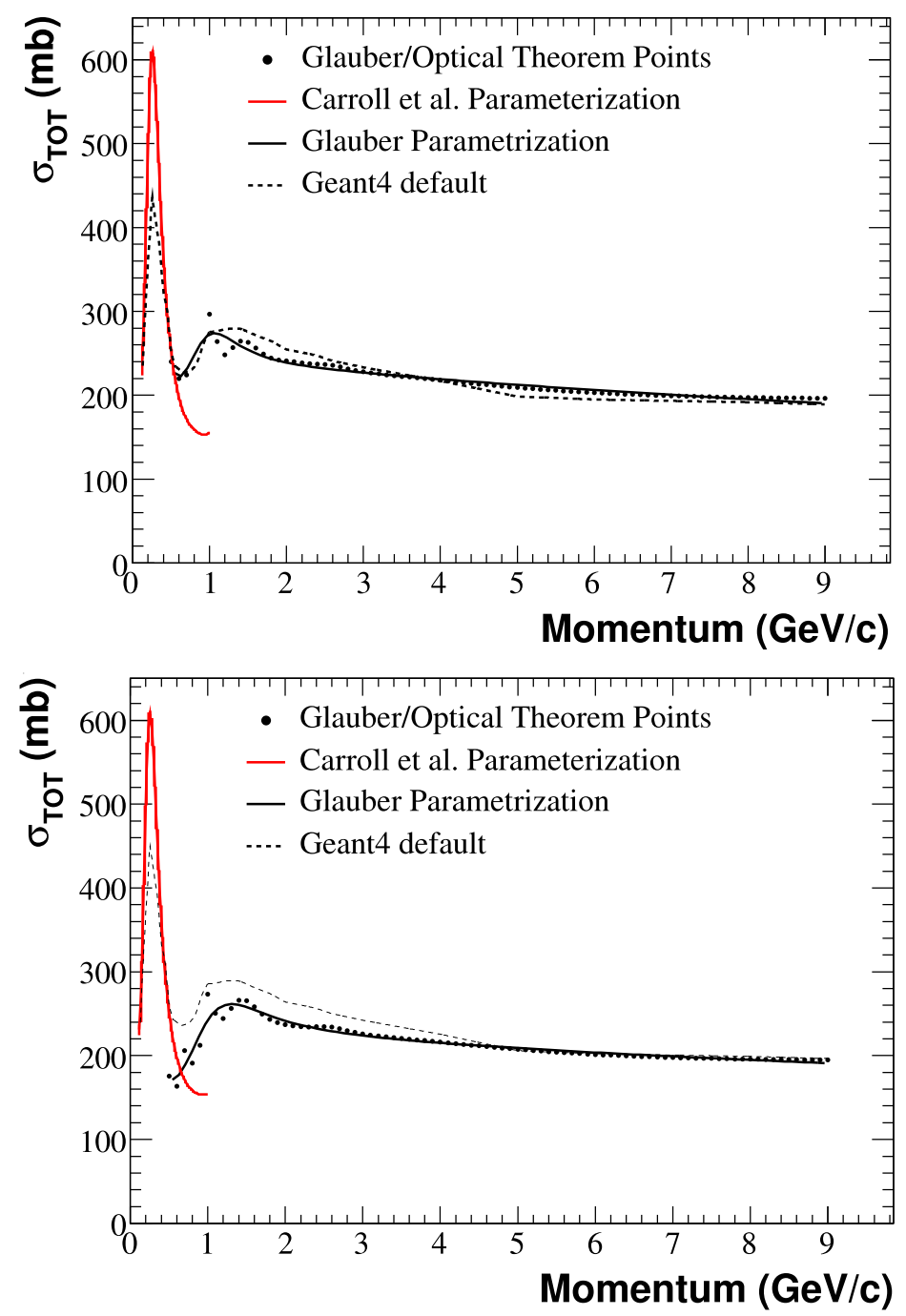

Figure 4.3: Total hadronic cross sections for $\pi^{+/-}$-Be calculated using the Glauber model (black points) for $\pi^{+}$(left) and $\pi^{-}$(right). The Breit-Wigner parametrization based on the Carroll data [50] on the $\Delta(1232)$ resonance is shown as a red line, while the parametrization of the Glauber model points is shown as a solid black line. The GEANT4 default model is shown as a dashed black line. 

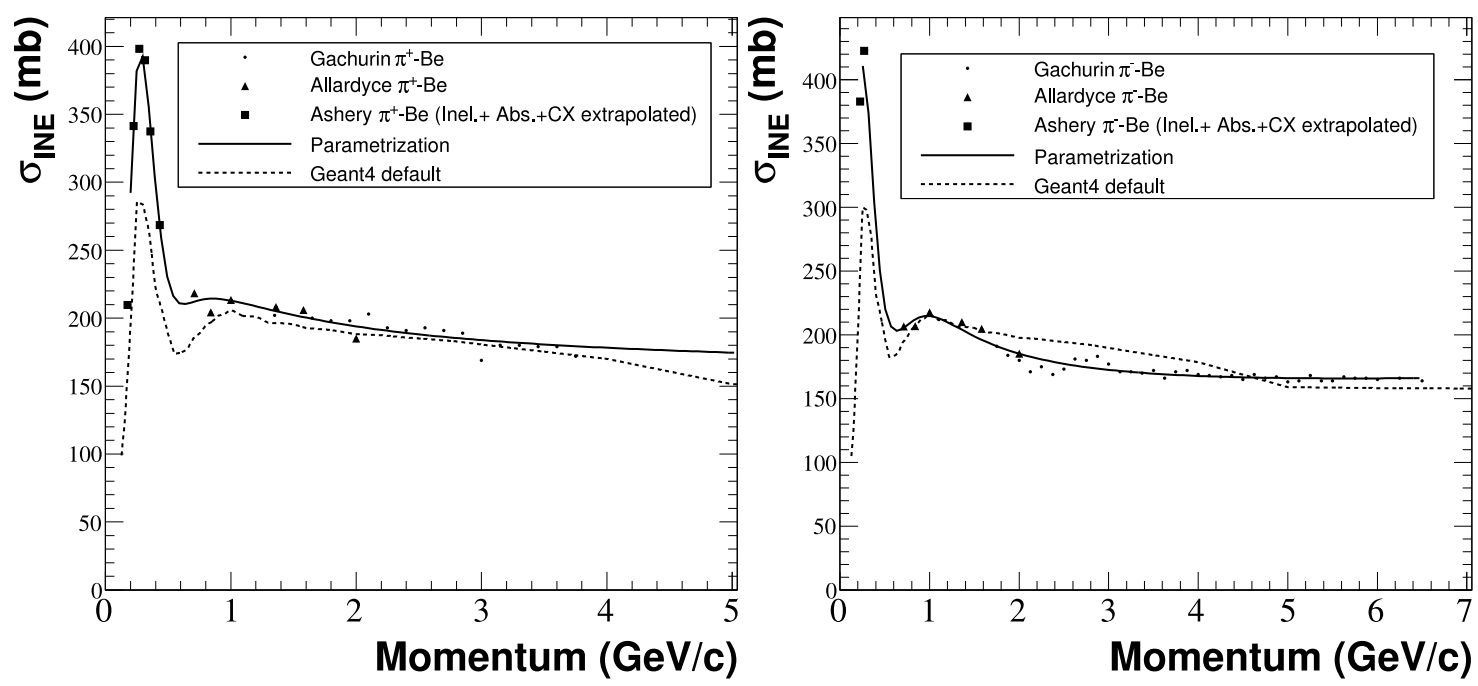

Figure 4.4: Inelastic cross sections for $\pi^{+}$-Be (top left), $\pi^{-}$-Be (top right), $\pi^{+}-\mathrm{Al}$ (bottom left) and $\pi^{-}-\mathrm{Al}$ (bottom right) as measured in References [51] (black squares), [52] (black triangles) and [53] (black circles). The solid black lines are the parameterizations used in the flux prediction, while the dashed lines are the default GEANT4 parameterizations.
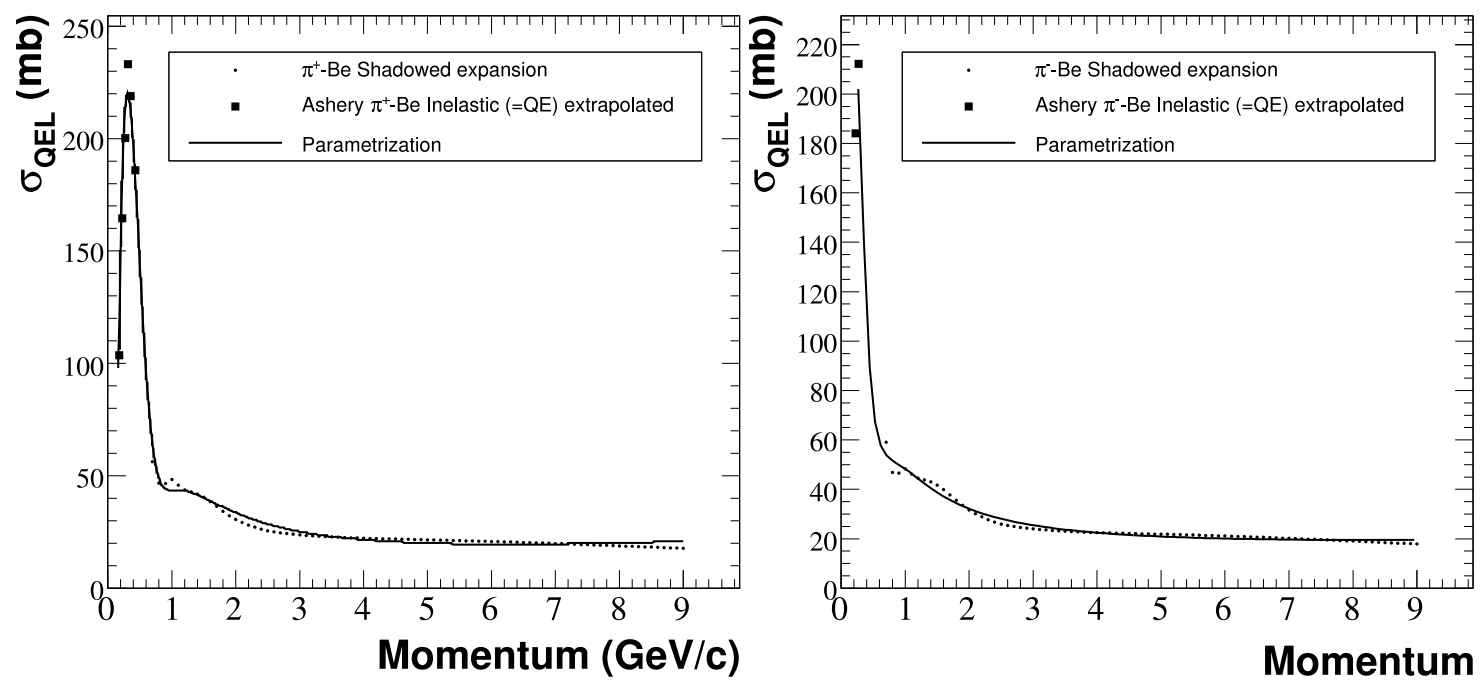

Figure 4.5: Quasi-elastic cross sections for $\pi^{+}$-Be (top left), $\pi^{-}$-Be (top right), $\pi^{+}$-Al (bottom left) and $\pi^{-}-\mathrm{Al}$ (bottom right) as measured in Ref. [51] (black squares) and calculated using the shadowed scattering model (black circles). The solid black lines are the parameterizations used in the flux prediction. 


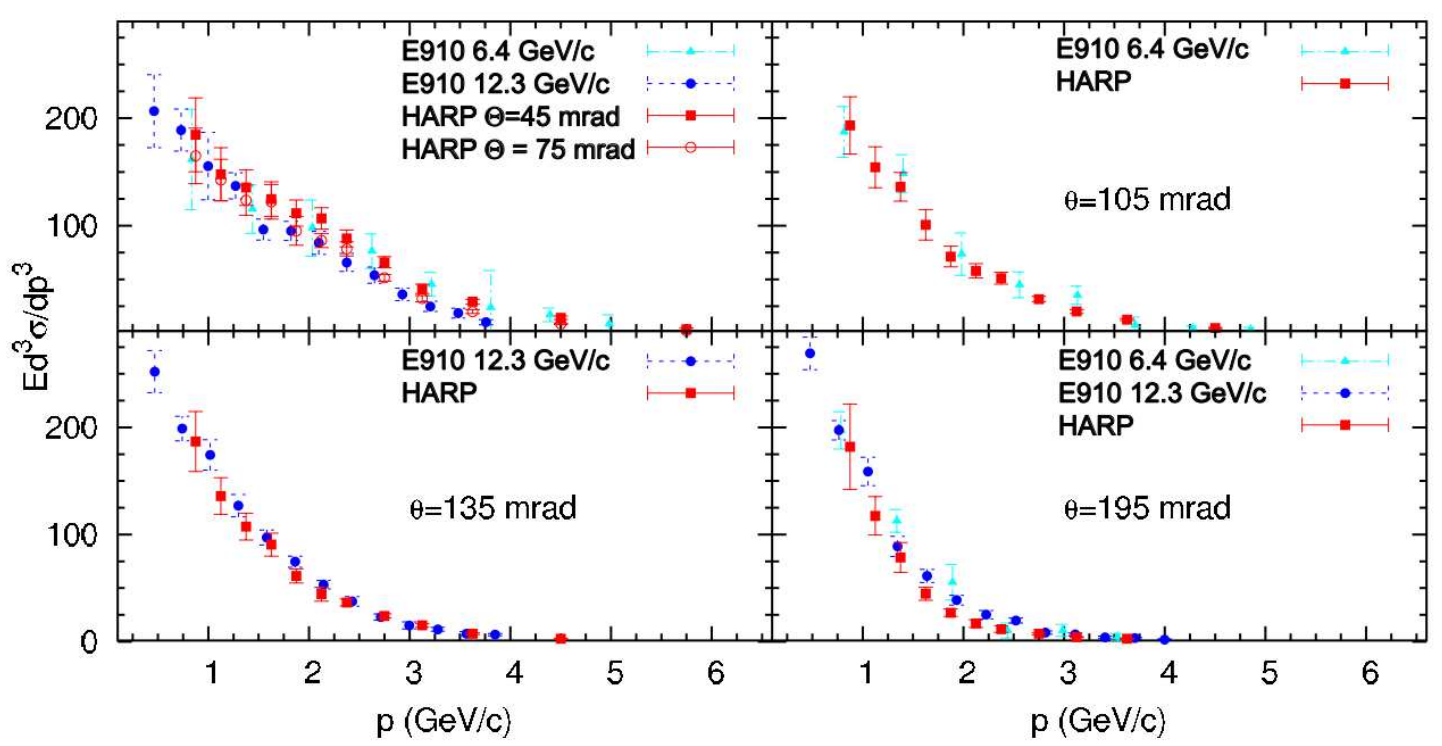

Figure 4.6: Invariant pion production cross section from HARP and E910 versus $p_{\pi}$ in bins of $\theta_{\pi}$. The E910 measurements are rescaled to $p_{B}=8.9 \mathrm{GeV} / \mathrm{c}$ by calculating $p_{T}$ and $x_{F}$ and then the appropriate $p_{\pi}, \theta_{\pi}$ assuming $p_{\text {beam }}=8.9 \mathrm{GeV} / \mathrm{c}$

data to produce a measurement of the differential cross section for kaon production, it has not been published yet. Therefore, the production cross section is determined from existing data on Be with different proton beam energies. Each data is scaled to $8.9 \mathrm{GeV} / \mathrm{c}$ using Feynman Scaling, which states that the invariant cross section should be a function of two variables, $p_{T}$ and $x_{F} \cdot p_{T}$ is the transverse component of the momentum of the outgoing meson, and $x_{F}=\frac{p_{\|}^{c m}}{p_{\|}^{\text {max }, c m}}$ is the Feynman Scaling variable. $p_{\|}^{c m}$ is the momentum parallel to the beam in the center of mass frame, and $p_{\|}^{\max , c m}$ is the maximum possible value for a given reaction. Figure 4.6 shows an example of Feynman Scaling with the pion production cross section in angular bins vs. pion momentum with E910 and HARP data. HARP data is produced with beam energy of $8.9 \mathrm{GeV} / \mathrm{c}$, but E910 is produced with beam energies of 6.4 and $12.3 \mathrm{GeV} / \mathrm{c}$. The $p_{T}$ and $x_{F}$ for the E910 data is calculated, and then the pion momentum and angle are calculated from $p_{T}$ and $x_{F}$, assuming a $8.9 \mathrm{GeV} / \mathrm{c}$ beam proton, as the cross section should just depend on those variables, not the beam momentum. The agreement in Fig. 4.6 between E910 and HARP confirms this assumption. 
The differential cross section for kaon production is parameterized by the following form which is derived from a parameterization of the invariant cross section in terms of the scaling variables, $x_{F}$ and $p_{T}$ :

$$
\begin{aligned}
\frac{d^{2} \sigma}{d p d \Omega}=\frac{p_{K^{+}}^{2}}{E_{K^{+}}}\left(E_{K^{+}} \frac{d^{3} \sigma}{d p_{K^{+}}^{3}}\right)= & \frac{p_{K^{+}}^{2}}{E_{K^{+}}} \times c_{1}\left(1-\left|x_{F}\right|\right) \times \\
& \exp \left[-c_{2} p_{T}-c_{3}\left|x_{F}\right|^{c_{4}}-c_{5} p_{T}^{2}-c_{7}\left|p_{T} \times x_{F}\right|^{c_{6}}\right]
\end{aligned}
$$

where $c_{i}$ are the fitted parameters and $p_{K}$ is the momentum of the kaon. Fig. 4.7 shows the data for kaon production all scaled to beam momentum $8.9 \mathrm{GeV} / \mathrm{c}$ along with the parameterization and uncertainty on the fit.

The data, listed in Table 4.2, is used to determine the $c_{i}$ of Eq. 4.3 in a similar manner as the pion data. The best fit values and correlation matrix of $c_{i}$ is shown in Table 4.3. The Vorontsov data had an inconsistent normalization with the other data sets, so its normalization uncertainty was increased to $500 \%$. The best fit $\chi^{2}$ for the parameterization is 2.28 , so the errors in the measurements are inflated by $\sqrt{2.28}=1.5$ so that the $\chi^{2} / D O F=1$.

The production of $K^{-}$and $K^{0}$ is also included in the beam simulation, but the predicted flux of neutrinos from either $K^{0}$ or $K^{-}$is $<0.25 \%$ of the total regardless of neutrino or antineutrino running.

\subsubsection{Horn Magnetic Field}

Before the mesons decay to neutrinos, they are focused by the strong magnetic field produced by the horn. Outside the inner conductor, the magnetic field has a $\frac{1}{r}$ dependence from the $174 \mathrm{kA}$ current which flows on the conductor. The strength of the magnetic field of the horn was measured, as shown in Fig. 4.8. Due to the pulsing of the horn, the current does not only flow on the surfaces of the horn, but instead some charge can "bleed" into the inner conductor surface. This behavior is the "skin effect", and the current

density is modeled to be an exponential decay into the inner conductor with a depth of $1.4 \mathrm{~mm}$. The uncertainty on the skin depth is taken to be the difference between the default (with skin depth effect present) and the current flowing only on the outer surface of the inner conductor, as no measurement of this could be physically made. The effect is a few 


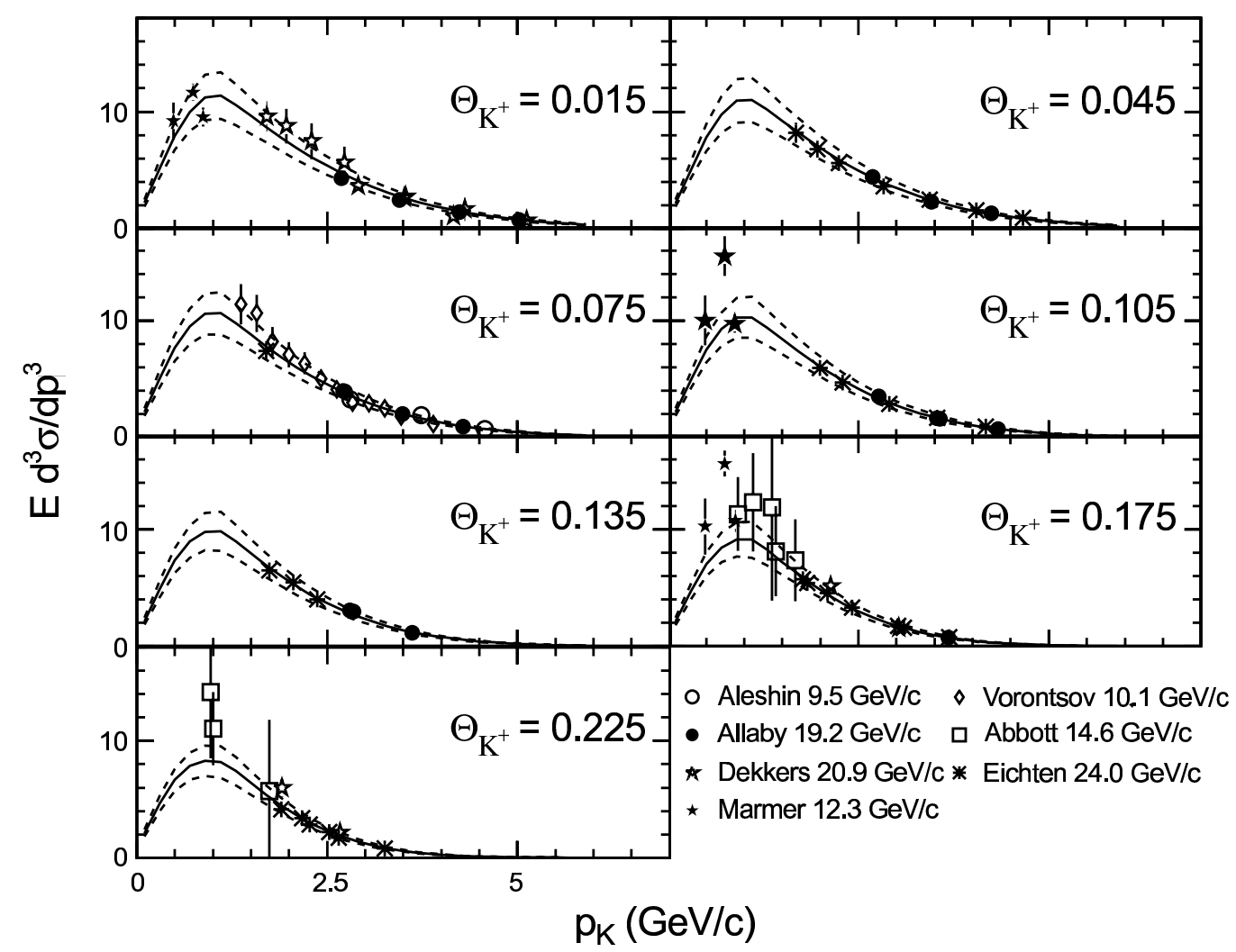

Figure 4.7: Comparison of $K^{+}$invariant cross section data (points) as a function of $p_{K^{+}}$, the $K^{+}$momentum, in bins of $\Theta_{K^{+}}$, the $K^{+}$production angle (in radians), with the Feynman Scaling based parametrization with best fit parameters shown as a solid line. The scaling has been used to relate the measurements at different primary beam momenta to the $8.9 \mathrm{GeV} / \mathrm{c}$ primary momentum in the BNB. The dashed lines represent the uncertainty band resulting from varying the parameters within their correlated uncertainties. The uncertainty bands include the factor 1.5 error inflation to set $\chi^{2} / \mathrm{DOF}=1$. 


\begin{tabular}{l|c|rcccc}
\hline \hline Dataset & $\begin{array}{c}p_{\text {beam }} \\
(\mathrm{GeV} / \mathrm{c})\end{array}$ & $\begin{array}{r}p_{K^{+}} \\
(\mathrm{GeV} / \mathrm{c})\end{array}$ & $\begin{array}{c}\theta_{K^{+}} \\
(\text {degrees })\end{array}$ & \multicolumn{2}{c}{$x_{F}$} & $\begin{array}{c}p_{T} \\
(\mathrm{GeV} / \mathrm{c})\end{array}$ \\
\hline Abbott [54] & 14.6 & $2-8$ & $20-30$ & $-0.12-0.07$ & $0.2-0.7$ & $10 \%$ \\
Aleshin [58] & 9.5 & $3-6.5$ & 3.5 & $0.3-0.8$ & $0.2-0.4$ & $10 \%$ \\
Allaby [55] & 19.2 & $3-16$ & $0-7$ & $0.3-0.9$ & $0.1-1.0$ & $15 \%$ \\
ekkers [56] & $18.8,23.1$ & $4-12$ & 0,5 & $0.1-0.5$ & $0.0-1.2$ & $20 \%$ \\
Eichten [57] & 24.0 & $4-18$ & $0-6$ & $0.1-0.8$ & $0.1-1.2$ & $20 \%$ \\
Vorontsov [59] & 10.1 & $1-4.5$ & 3.5 & $0.03-0.5$ & $0.1-0.25$ & $25 \% \rightarrow 500 \%$ \\
\hline
\end{tabular}

Table 4.2: Summary of $K^{+}$production measurements in p-Be interactions used to characterize $K^{+}$production in the BNB. The table includes $p_{\text {beam }}$, the primary proton momenta in the measurement, the momentum and angular ranges of the measurements, as well as the corresponding ranges of the Feynman Scaling variable $x_{F}$ and transverse momentum $p_{T}$. The quoted normalization uncertainty $\sigma_{N}$ is also listed for each experiment.

\begin{tabular}{c|rrrrrrr}
\hline \hline & $c_{1}$ & $c_{2}$ & $c_{3}$ & $c_{4}$ & $c_{5}$ & $c_{6}$ & $c_{7}$ \\
\hline Value & 11.70 & 0.88 & 4.77 & 1.51 & 2.21 & 2.17 & 1.51 \\
\hline$c_{1}$ & 1.094 & 0.0502 & $2.99 \times 10^{-3}$ & -0.0332 & -0.0375 & 0.125 & 0.0743 \\
$c_{2}$ & 0.0502 & 0.01610 & $1.39 \times 10^{-3}$ & $-1.44 \times 10^{-3}$ & -0.0126 & 0.0322 & 0.0220 \\
$c_{3}$ & $2.99 \times 10^{-3}$ & $1.39 \times 10^{-3}$ & $7.47 \times 10^{-3}$ & $2.06 \times 10^{-3}$ & $1.93 \times 10^{-3}$ & 0.0135 & $-3.34 \times 10^{-3}$ \\
$c_{4}$ & -0.0332 & $-1.44 \times 10^{-3}$ & $2.06 \times 10^{-3}$ & $3.46 \times 10^{-3}$ & $2.03 \times 10^{-3}$ & $-4.11 \times 10^{-3}$ & $-6.28 \times 10^{-3}$ \\
$c_{5}$ & -0.0375 & -0.0126 & $1.93 \times 10^{-3}$ & $2.03 \times 10^{-3}$ & 0.0146 & -0.0154 & -0.0244 \\
$c_{6}$ & 0.0125 & 0.0322 & 0.0135 & $-4.11 \times 10^{-3}$ & -0.0154 & 0.0182 & 0.0126 \\
$c_{7}$ & 0.0743 & 0.0220 & $-3.34 \times 10^{-3}$ & $-6.28 \times 10^{-3}$ & -0.0244 & 0.126 & 0.159 \\
\hline
\end{tabular}

Table 4.3: Best fit Feynman Scaling model parameters $c_{i}$ from a fit to $K^{+}$production data (first row). The covariance matrix for the parameters with uncertainties inflated by a factor of 1.5 to set $\chi^{2} / \mathrm{DOF}=1$ is in the table below the parameters. 


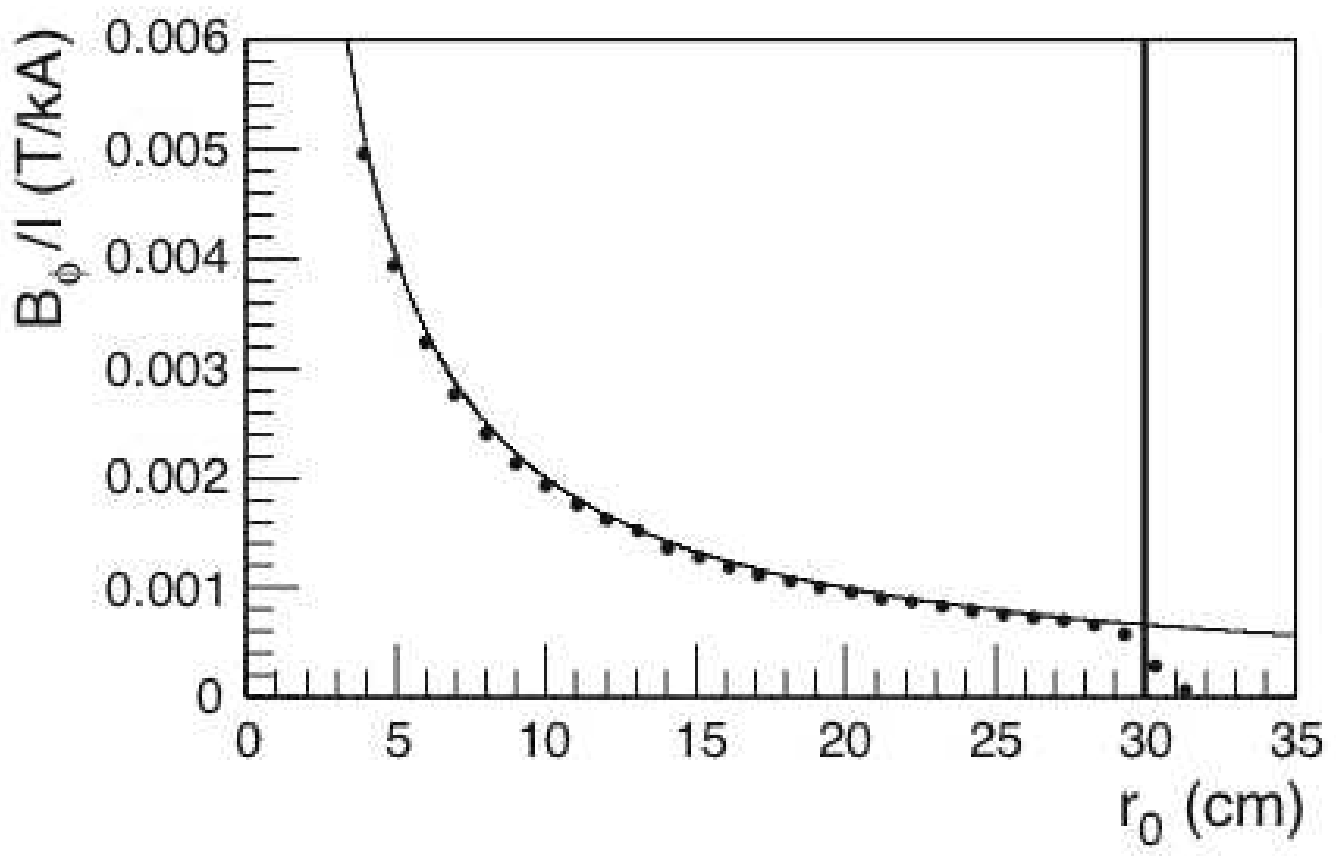

Figure 4.8: Measurements of the azimuthal magnetic field within the horn. The points show the measured magnetic field, while the line shows the expected $1 / r$ dependence. The black vertical line indicates the outer radius of the horn. 
percent for most of the flux, but at high energies the mesons which produce neutrinos travel substantially through this region of the magnetic field.

The second uncertainty on the magnetic field comes from the absolute scale of the horn current. Fig. 4.9 shows the horn current for the entire MiniBooNE neutrino run, along with two bands for the assumed horn current uncertainty of \pm 1 kA. Fig. 4.10 shows the entire antineutrino run through March 12th, 2009.

\subsubsection{Meson Decays to Neutrino Beam}

Finally, a simulation is made of the meson decay to neutrinos. The simulation uses the known lifetime and branching ratios for $\pi$ and $K^{+}$[60]. While theoretically one can produce enough neutrinos by simulating many proton interactions, it is often much faster and easier to rely on statistical boosting techniques. Two are used in the beamline simulation: redecay and high momentum weighting. First, each meson is decayed $\sim 1000$ times ("redecayed") to produce a set of neutrinos, each with different kinematics. Second, the long tail at high energy of the BNB flux makes it hard to populate the space of high energy neutrinos; high energy mesons will preferentially send most of the redecayed neutrinos at the detector, but few of these parents will exist. To correct for this, the meson production cross section is given a weight based on the longitudinal meson momentum. Each daughter event is then down-weighted accordingly, providing a larger space of neutrino parents and a more accurate neutrino flux at high energies.

\subsubsection{Neutrino and Antineutrino Fluxes}

The result of the neutrino beam simulation is shown at a $610 \mathrm{~cm}$ radius sphere centered at $541 \mathrm{~m}$ (MiniBooNE) in Fig. 4.11, and at a $212 \mathrm{~cm}$ radius sphere centered on $100 \mathrm{~m}$ (SciBooNE) in Fig. 4.12. The corresponding plots for antineutrino fluxes are shown in Fig. 4.13 and 4.14 for MiniBooNE and SciBooNE respectively. The fluxes at a sphere centered on each detector are very similar, the main differences in the parentage of events in MiniBooNE and SciBooNE arise from the size and acceptance of the two detectors. 
Average horn current (kA)
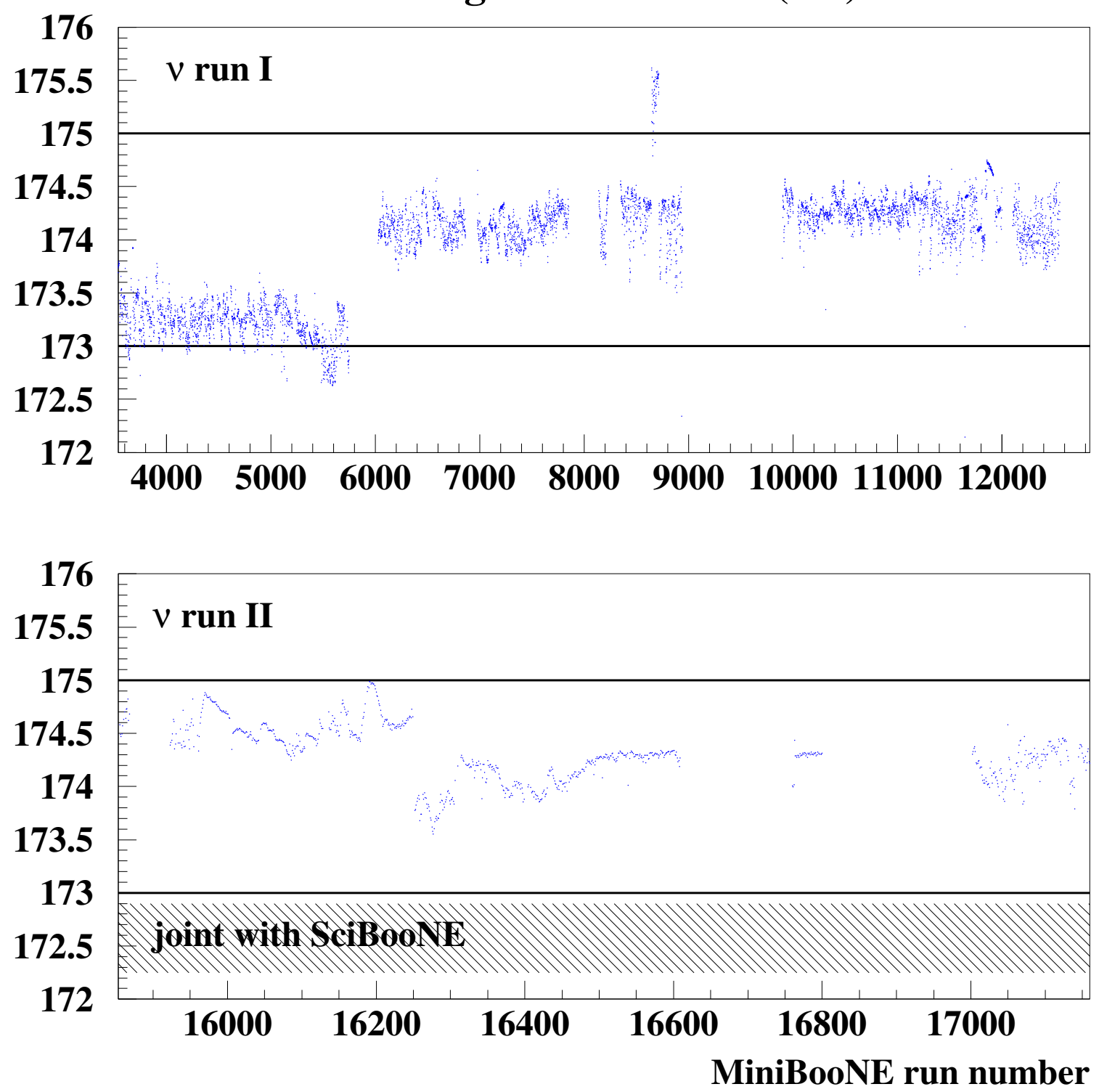

Figure 4.9: Current (kA) on the horn vs. MiniBooNE run number for neutrino mode. The black lines denote the $\pm 1 \mathrm{kA}$ uncertainty. Also noted are the joint SciBooNE run periods with hashed boxes. 
Average horn current (kA)
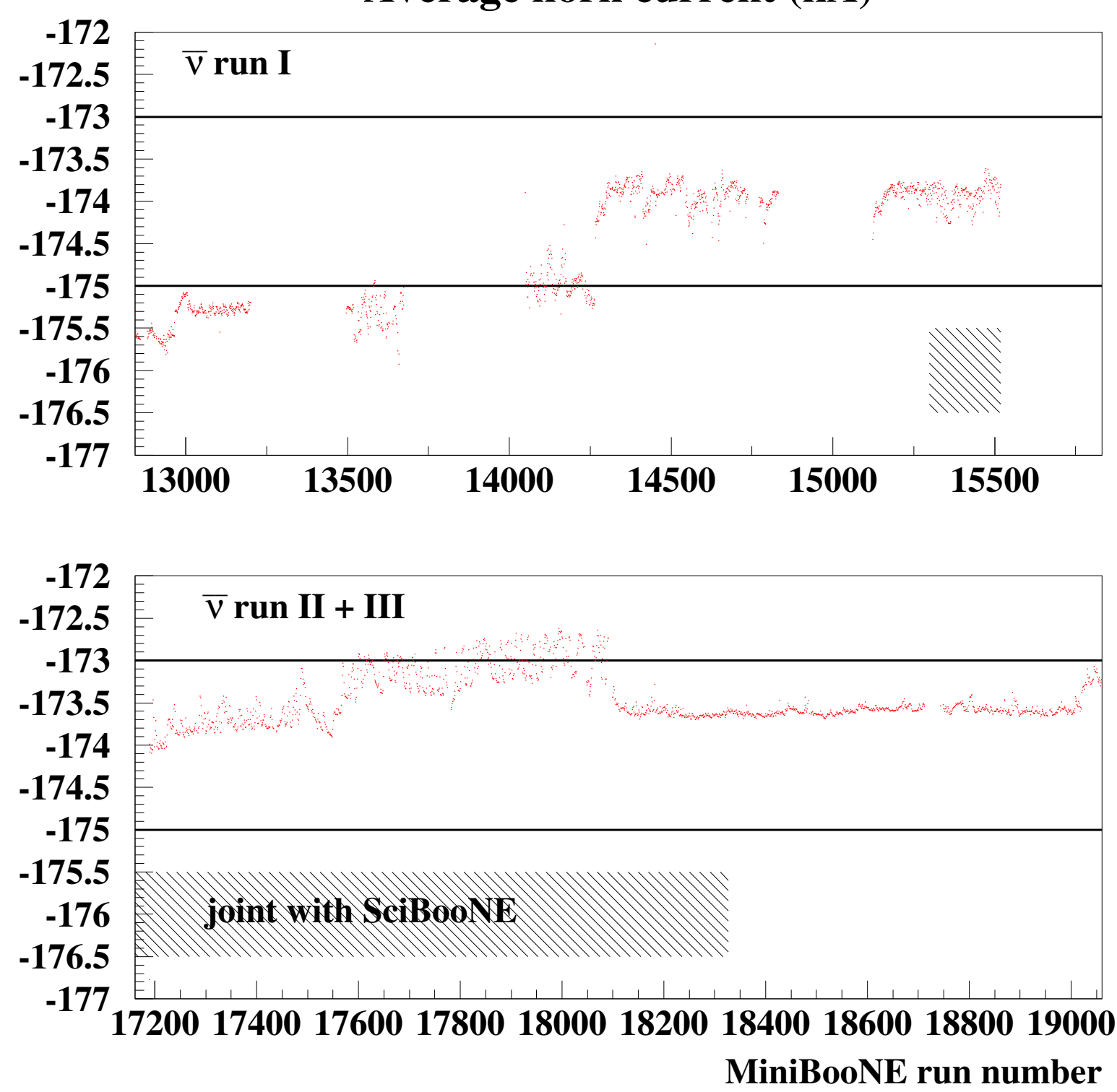

Figure 4.10: Current (kA) on the horn vs. MiniBooNE run number for antineutrino mode. The black lines denote the $\pm 1 \mathrm{kA}$ uncertainty. Also noted are the joint SciBooNE run periods with hashed boxes. 

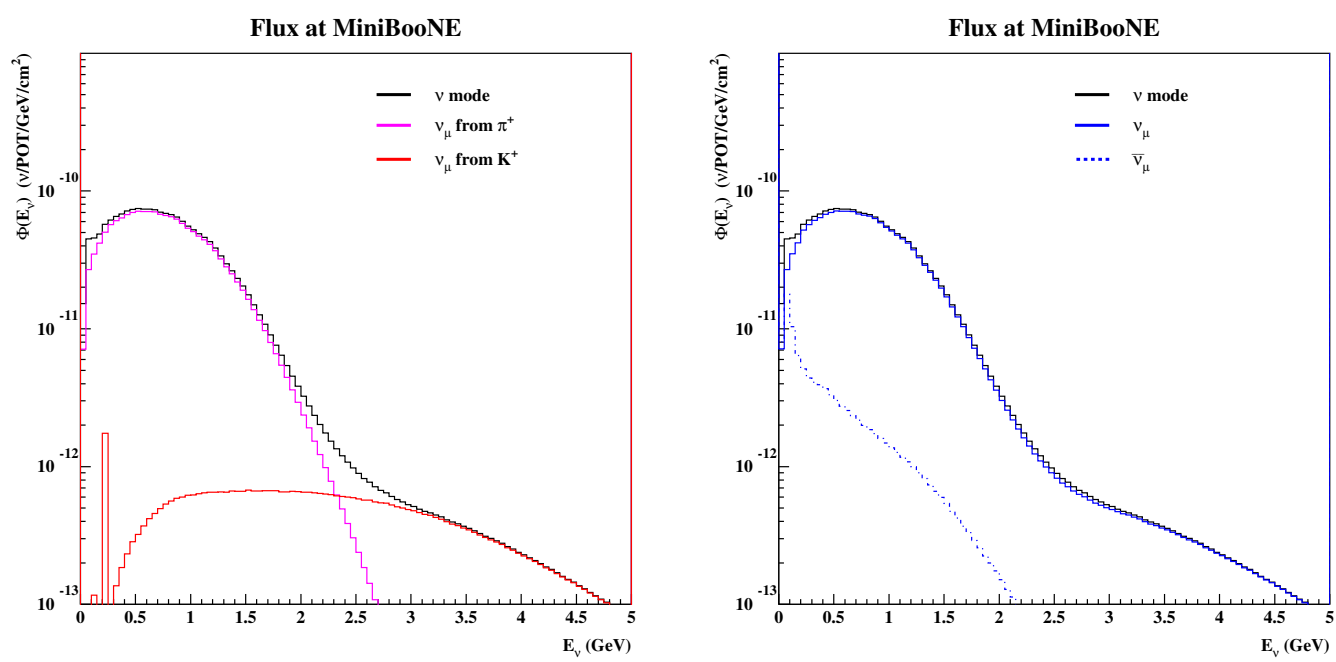

Figure 4.11: Predicted neutrino mode flux at MiniBooNE $(610 \mathrm{~cm}$ sphere at $541 \mathrm{~m}$ from the target) Left: Total flux (black) shown with flux by parent pion (purple) and kaon (red). Right: Total flux (black) shown with flux by neutrino type, neutrinos (solid blue) and antineutrino (dashed blue).
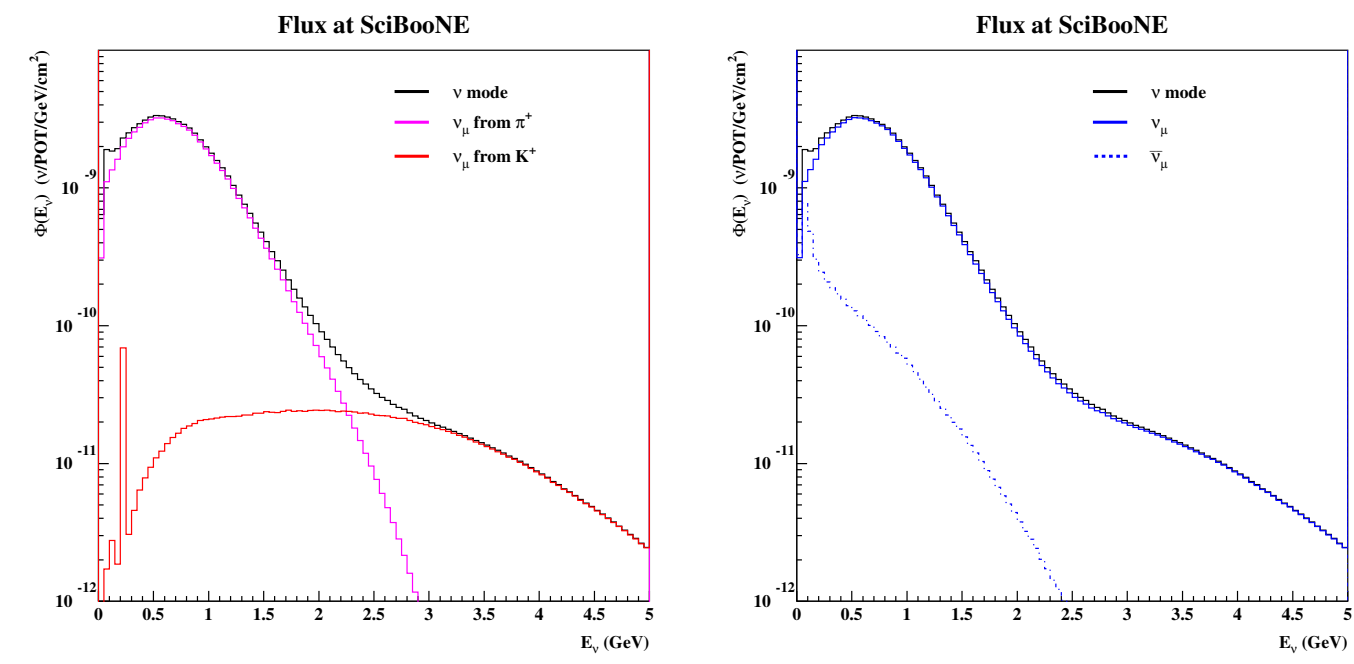

Figure 4.12: Predicted neutrino mode flux at SciBooNE $(212 \mathrm{~cm}$ sphere at $100 \mathrm{~m}$ from the target) Left: Total flux (black) shown with flux by parent pion (purple) and kaon (red). Right: Total flux (black) shown with flux by neutrino type, neutrinos (solid blue) and antineutrino (dashed blue). 

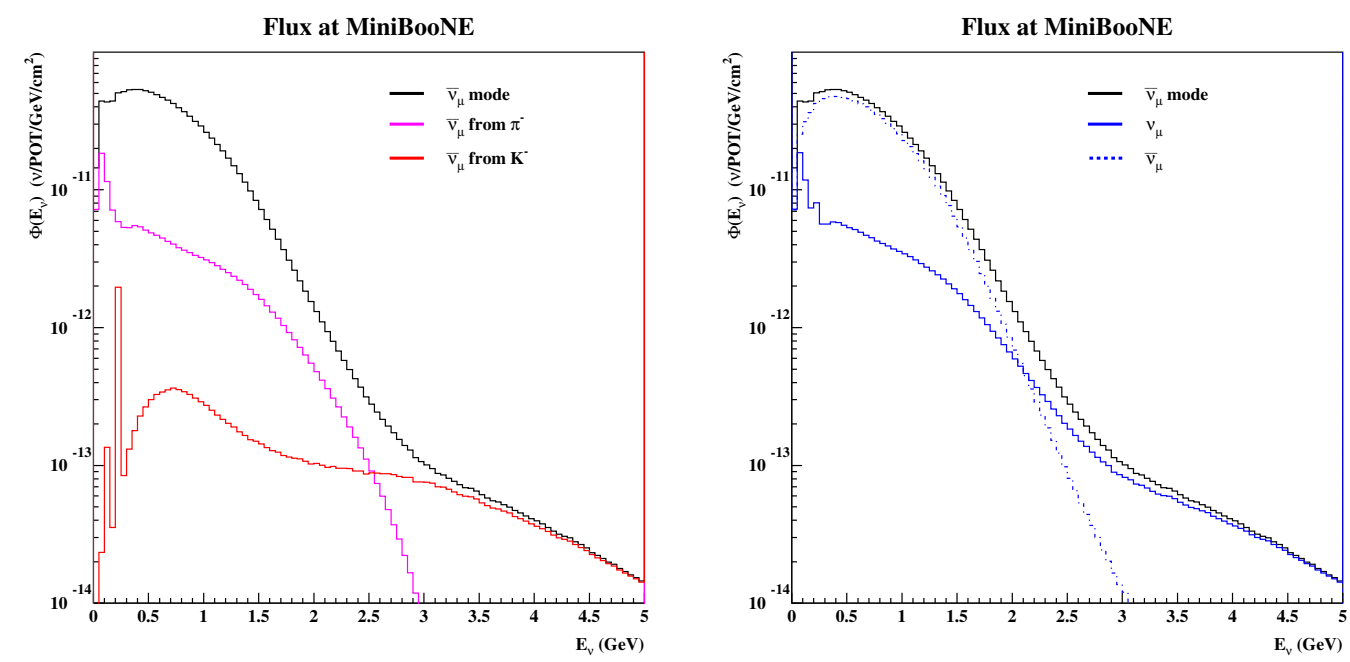

Figure 4.13: Predicted antineutrino mode flux at MiniBooNE $(610 \mathrm{~cm}$ sphere at $541 \mathrm{~m}$ from the target) Left: Total flux (black) shown with flux by parent pion (purple) and kaon (red). Right: Total flux (black) shown with flux by neutrino type, neutrinos (solid blue) and antineutrino (dashed blue).
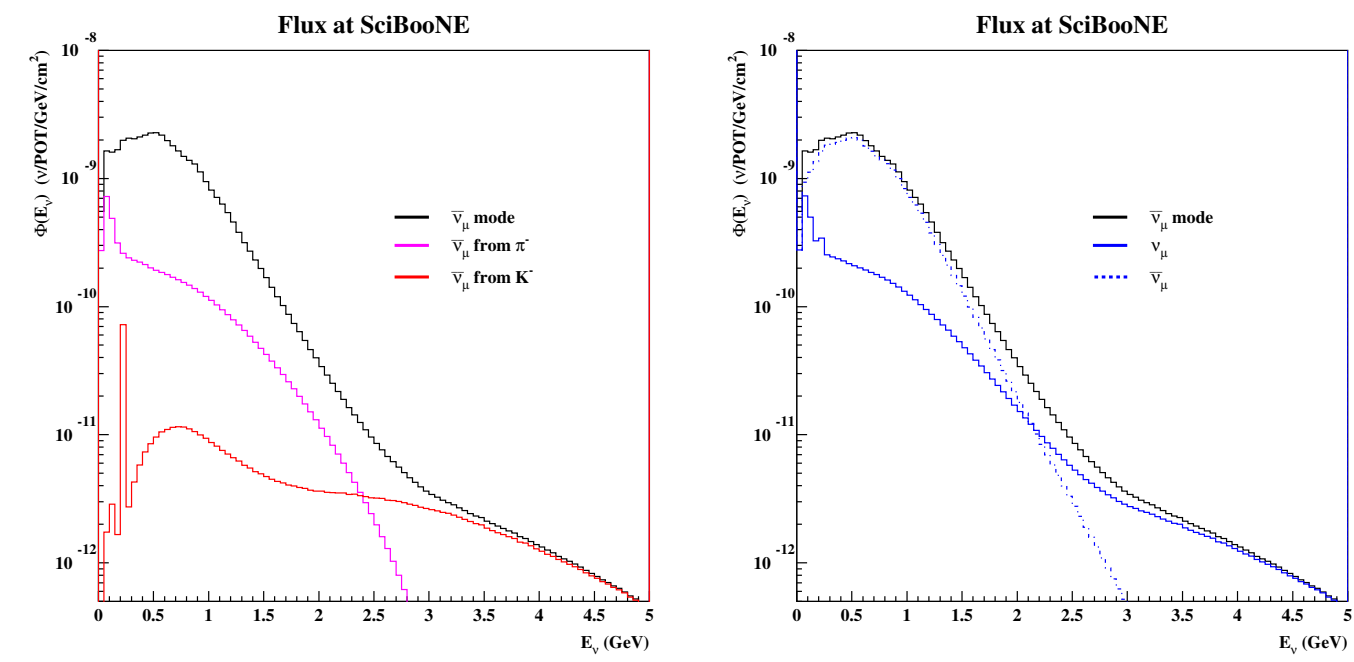

Figure 4.14: Predicted antineutrino mode flux at SciBooNE $(212 \mathrm{~cm}$ sphere at $100 \mathrm{~m}$ from the target) Left: Total flux (black) shown with flux by parent pion (purple) and kaon (red). Right: Total flux (black) shown with flux by neutrino type, neutrinos (solid blue) and antineutrino (dashed blue). 

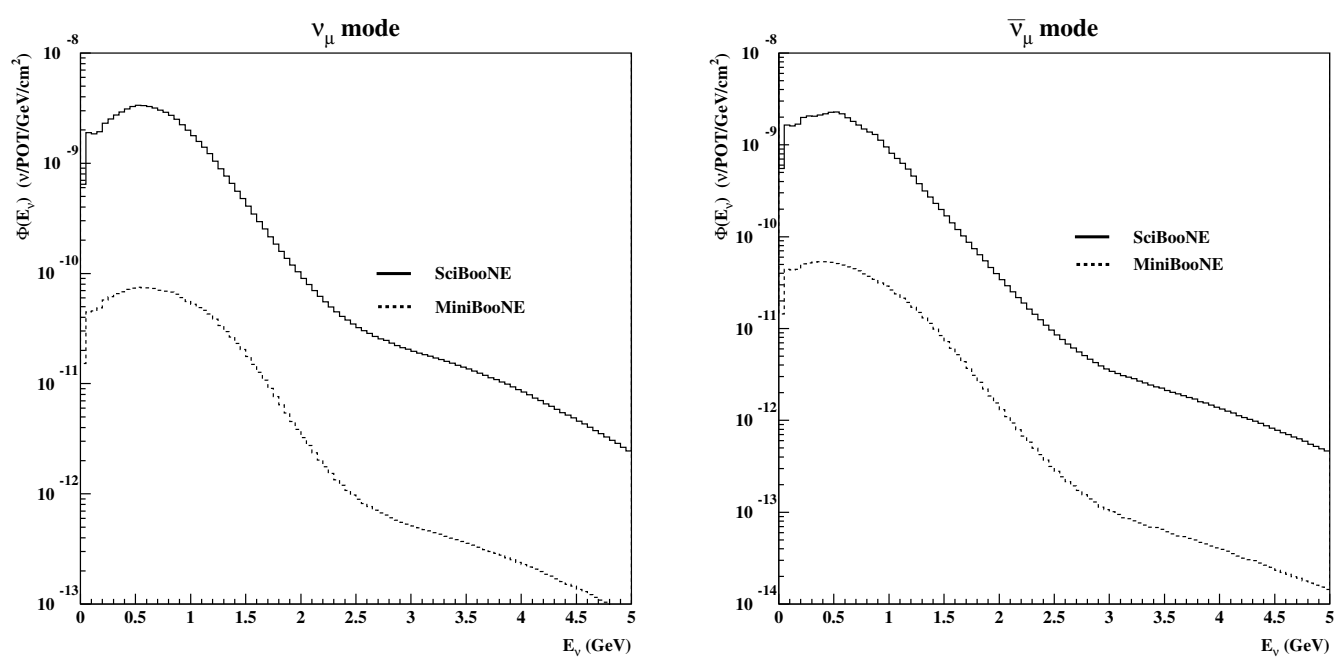

Figure 4.15: Predicted flux at SciBooNE (212 cm sphere at $100 \mathrm{~m}$ from the target) and MiniBooNE (610 cm sphere at $541 \mathrm{~m}$ from the target) vs. neutrino energy $(\mathrm{GeV})$. Left: Total neutrino mode flux at SciBooNE (black) shown with MiniBooNE (dash). Right: Total antineutrino mode flux at SciBooNE (black) shown with MiniBooNE (dash).

\subsection{Neutrino Interactions}

The total neutrino charged current (CC) cross section at the BNB flux energy (200 $\mathrm{MeV}$ to $2 \mathrm{GeV}$ ) is shown in Fig. 4.16. Below $\sim 1 \mathrm{GeV}$, neutrinos interact via charged current quasi-elastic (CCQE) scattering. As the neutrinos increase in energy, neutrino interactions have a chance to produce additional particles, like charged current single pion $(\mathrm{CC} \pi)$ production, and eventually multiple particles via deep inelastic scattering (DIS). The antineutrino total CC cross section is shown in Fig. 4.17.

In MiniBooNE and SciBooNE, approximately $42 \%$ of all events are due to CCQE interactions, $22 \%$ are due to $\mathrm{CC} \pi^{+/-}$interactions and $4 \%$ are due to $\mathrm{CC} \pi^{0}$ interactions. Neutral current processes account for the rest of the interactions, while DIS and muliti-pion production are less than $\sim 1 \%$ of all interactions.

In both experiments, the NUANCE [81] neutrino interaction generator is used with the defaults mentioned in the text. 


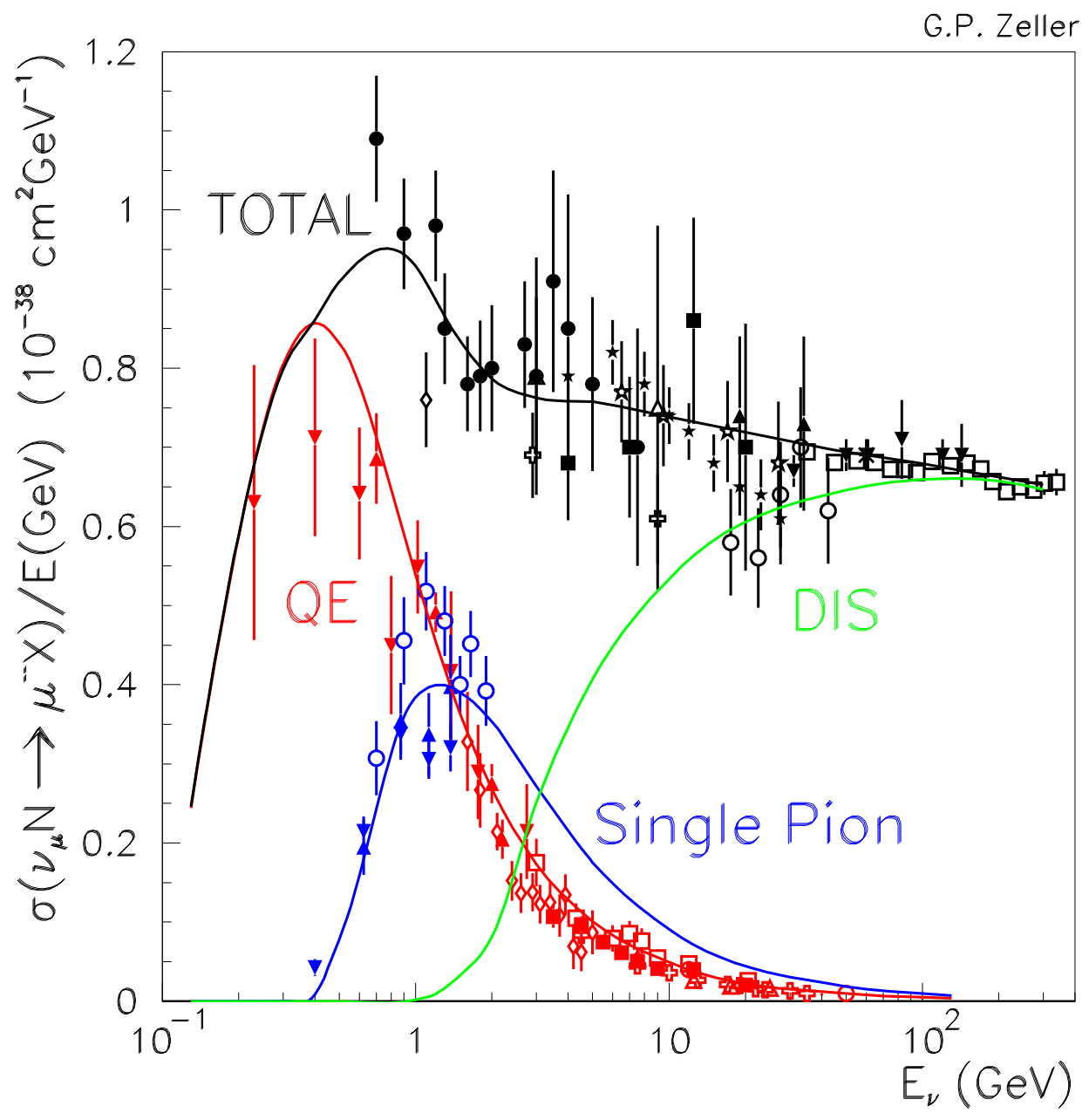

Figure 4.16: World's existing published data $[61,62,63,64,65,66,67,68,69,70,71,72$, $73,74,75,76,77]$ on neutrino CC cross sections. Plotted is the CC cross section divided by neutrino energy, as a function of neutrino energy. CCQE is shown in red, CC $\pi$ in blue and DIS in green. Also plotted is the prediction (solid line with corresponding color). 


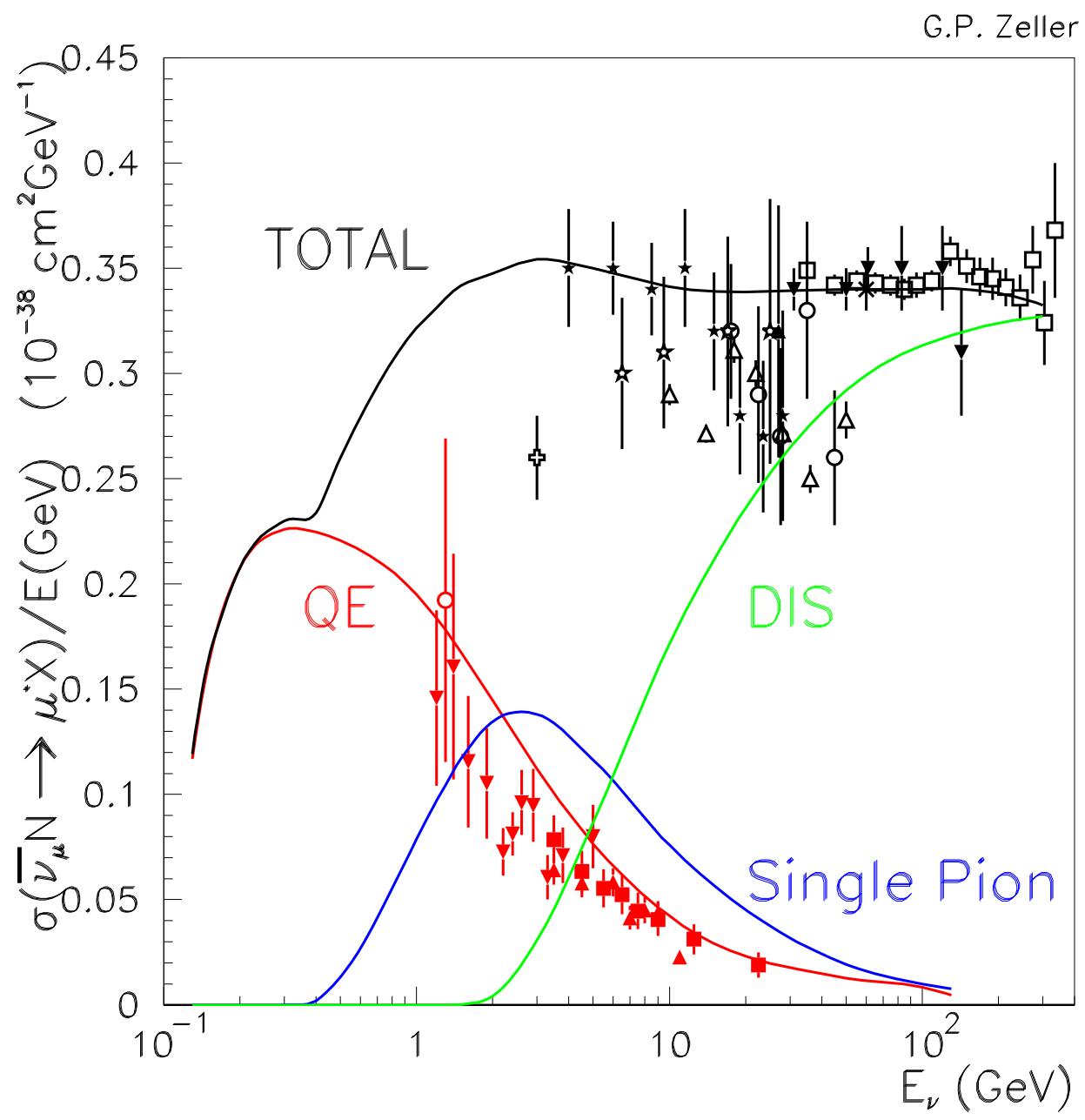

Figure 4.17: World's existing published data $[70,71,72,73,74,75,76,77,78,79,80]$ on antineutrino CC cross sections. Plotted is the CC cross section divided by neutrino energy as a function of neutrino energy. CCQE is shown in red, CC $\pi$ in blue and DIS in green. Also plotted is the prediction (solid line with corresponding color). 
neutrino-bound nucleon scattering

(1) Lab frame

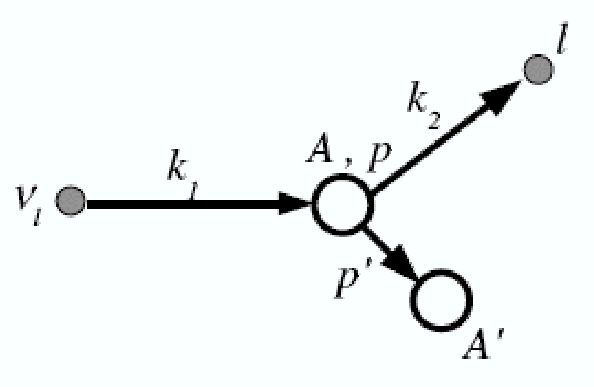

(2) Feynman diagram

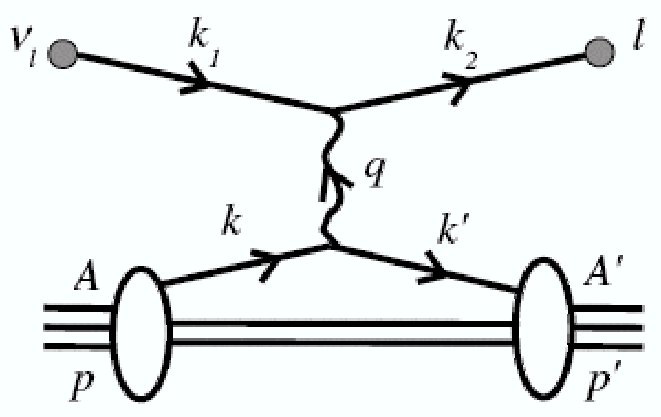

Figure 4.18: The CCQE interaction in the lab frame and the Feynman diagram for the interaction. $k_{1}$ is the incident neutrino 4 -vector, $k_{2}$ is the outgoing lepton's 4 -vector, and $q=k_{1}-k_{2} . A$ is carbon for both MiniBooNE and SciBooNE, and $p$ denotes the nucleon involved.

\subsubsection{CCQE Scattering}

CCQE scattering with neutrinos $\left(\nu^{l}+n \rightarrow l^{-}+p\right)$ or antineutrinos $\left(\bar{\nu}^{l}+p \rightarrow l^{+}+n\right)$ is the process used to search for oscillations at $\sim 1 \mathrm{GeV}$ because of its simple final state. It is the most abundant process at $\sim 1 \mathrm{GeV}$, and, as shown later in Section 5 , it is relatively easy to get a high purity CCQE sample ( 70\%).

The CCQE cross section was first described by Llwellyn-Smith [82]:

$$
\frac{d^{2} \sigma(C C Q E \nu / \bar{\nu})}{d Q^{2}}=\frac{M^{2} G_{F}^{2} \cos ^{2}\left(\theta_{c}\right)}{8 \pi E_{\nu}^{2}}\left[A\left(Q^{2}\right) \pm B\left(Q^{2}\right) \frac{(s-u)}{M^{2}}+C\left(Q^{2}\right) \frac{(s-u)^{2}}{M^{4}}\right]
$$

where $M$ is the nucleon mass, $G_{F}$ is the Fermi coupling (strength of the weak force), $\theta_{c}$ is the Cabbibo angle, $s$ and $u$ are Mandelstam variables with $s-u=4 M E_{\nu}-Q^{2}-m_{l}$, $Q^{2}$ is the 4-momentum transfered between the neutrino and the outgoing lepton. The derivation can be found under Ref. [5]. The sign difference between neutrino (+) and antineutrino (-) is because of the axial-vector current interference term $\left(\mathrm{B}\left(Q^{2}\right)\right)$ discussed below. This is a consequence of the V-A nature of the weak interactions. 
$\mathrm{A}, \mathrm{B}$, and $\mathrm{C}$ are functions of $Q^{2}$ defined in the following way:

$$
\begin{gathered}
A\left(Q^{2}\right)=\frac{m^{2}+Q^{2}}{M^{2}}\left[(1+\tau) F_{A}^{2}-(1-\tau) F_{1}^{2}+\tau(1-\tau) F_{2}^{2}+4 \tau F_{1} F_{2}\right. \\
\left.-\frac{m^{2}}{4 M^{2}}\left(F_{1}+F_{2}\right)^{2}+\left(F_{A}+2 F_{p}\right)^{2}-4 F_{p}^{2}(1+\tau)\right] \\
B\left(Q^{2}\right)=\frac{Q^{2}}{M^{2}} F_{A}\left(F_{1}+F_{2}\right) \\
C\left(Q^{2}\right)=\frac{1}{4}\left(F_{A}^{2}+F_{1}^{2}+\tau F_{2}^{2}\right)
\end{gathered}
$$

where $\tau=\frac{Q^{2}}{4 M^{2}}, F_{1,2}$ are the vector form factors, $F_{A}$ is the axial vector form factor and $F_{p}$ is the pseudo scalar form factor.

Assuming conserved vector current (CVC), the vector form factors are defined as:

$$
\begin{gathered}
F_{1}\left(Q^{2}\right)=\frac{1}{(1+\tau)}\left(G_{E}^{p}-G_{E}^{n}-\tau\left(G_{M}^{p}-G_{M}^{n}\right)\right) \\
\left.F_{1}\left(Q^{2}\right)=\frac{1}{1+\tau}\left(G_{M}^{p}-G_{M}^{n}-G_{E}^{p}+G_{E}^{n}\right)\right)
\end{gathered}
$$

The Sachs form factors $\left(G_{E, M}^{p, n}\right)$ are also present in electron scattering. Conventionally, the form factors are assumed to be a "dipole" format:

$$
G_{E, M}^{p, n}\left(Q^{2}\right)=\frac{G_{E, M}^{p, n}(0)}{1+\frac{Q^{2}}{M_{V}^{2}}}
$$

where $G_{E}^{p}(0)=1$, the proton electric charge, $G_{E}^{n}(0)=0$, the neutron electric charge, $G_{M}^{p}(0)=2.793$, the proton magnetic moment, and $G_{M}^{n}(0)=-1.913$, the neutron magnetic moment. The vector mass is $M_{V}^{2}=0.71 \mathrm{GeV}^{2}$.

Recent electron scattering data show deviations from the dipole form at high $Q^{2}$, and so these form factors are parameterized as functions of $Q^{2}$ from fits [83] to electron scattering data $[84,85,86,87]^{1}$

The axial form factor is assumed to be a dipole as well:

$$
F_{A}\left(Q^{2}\right)=\frac{-1.267}{1+\frac{Q^{2}}{M_{A}^{2}}}
$$

where $M_{A}$ is the axial mass for the CCQE interaction.

The pseudo scalar form factor is:

$$
F_{p}\left(Q^{2}\right)=\frac{2 M^{2}}{m_{\pi}^{2}+Q^{2}} F_{A}\left(Q^{2}\right)
$$

\footnotetext{
${ }^{1}$ While I was working on $\mathrm{K} 2 \mathrm{~K}$, we examined the effect of switching from a dipole form to a parameterization for the vector form factors. The effect on the CCQE cross section was about $2 \%$, lower at low $Q^{2}$ and higher at high $Q^{2}[88]$.
} 
where $m_{\pi}$ is the mass of the pion. For a discussion of the relationship between $F_{A}$ and $F_{p}$, see Ref. [5].

\subsubsection{Nuclear Effects}

In reality, the neutrino does not just interact on a proton or neutron, but instead it hits a nuclear target. In the case of both MiniBooNE and SciBar, the target is carbon. This was first derived by Smith and Moniz [89], with the derivation given under Ref. [5]. The formula includes hadronic nuclei functions which depend on the form factors mentioned above, and also the distribution $f$ of the nuclei in momentum space $\left(\int d \vec{k} f(\vec{k}, \vec{q}, \omega)\right)$.

If the target is assumed to be a relativistic Fermi Gas (RFG) characterized by Fermi momentum $p_{F}$ and nucleon binding energy $E_{B}$, then the integrals are analytically solvable:

$$
\int d \vec{k} f(\vec{k}, \vec{q}, \omega) \sim \int_{E_{l o}}^{E_{h i}} E d E
$$

where $E_{l o}=\kappa \sqrt{p_{F}^{2}+M_{n}^{2}}$ and $E_{h i}=\sqrt{p_{F}^{2}+M_{p}^{2}}-\omega+E_{B}$. The factor $\kappa$ represents a scale factor to increase or decrease the allowed momentum space, and therefore increase or decrease the amount of Pauli blocking. $E_{B}=34 \pm 9 \mathrm{MeV}$ and $p_{F}=220 \pm 30 \mathrm{MeV} / \mathrm{c}$ are measured from electron scattering data on carbon.

Using a different model than RFG of the nucleus, such as the spectral function model [90], reduces the CCQE cross by 10\%, independant of neutrino energy; this is included in the uncertainties on the CCQE cross section.

The only free parameters in the CCQE cross section are $M_{A}$ and $\kappa$. The world's data on neutrino interactions on deuterium gives $M_{A}=1.015 \mathrm{GeV}$ [91], however recent results from the K2K experiment on carbon [92] and oxygen [93] suggest a higher effective value of $M_{A}=1.2 \mathrm{GeV}$ for nuclear targets. In a shape-only fit [94] in $Q^{2}$, MiniBooNE data also favors a higher value of $M_{A}=1.23 \mathrm{GeV}$. The parameter $\kappa$ was introduced to correct for additional differences at low $Q^{2}$. Previous measurements did not fit below $Q^{2}<0.2[92,93]$. At energies higher than MiniBooNE and $\mathrm{K} 2 \mathrm{~K}$, the Nomad experiment measures $M_{A}=1.05 \pm 0.06 \mathrm{GeV}$ on carbon [95]. 


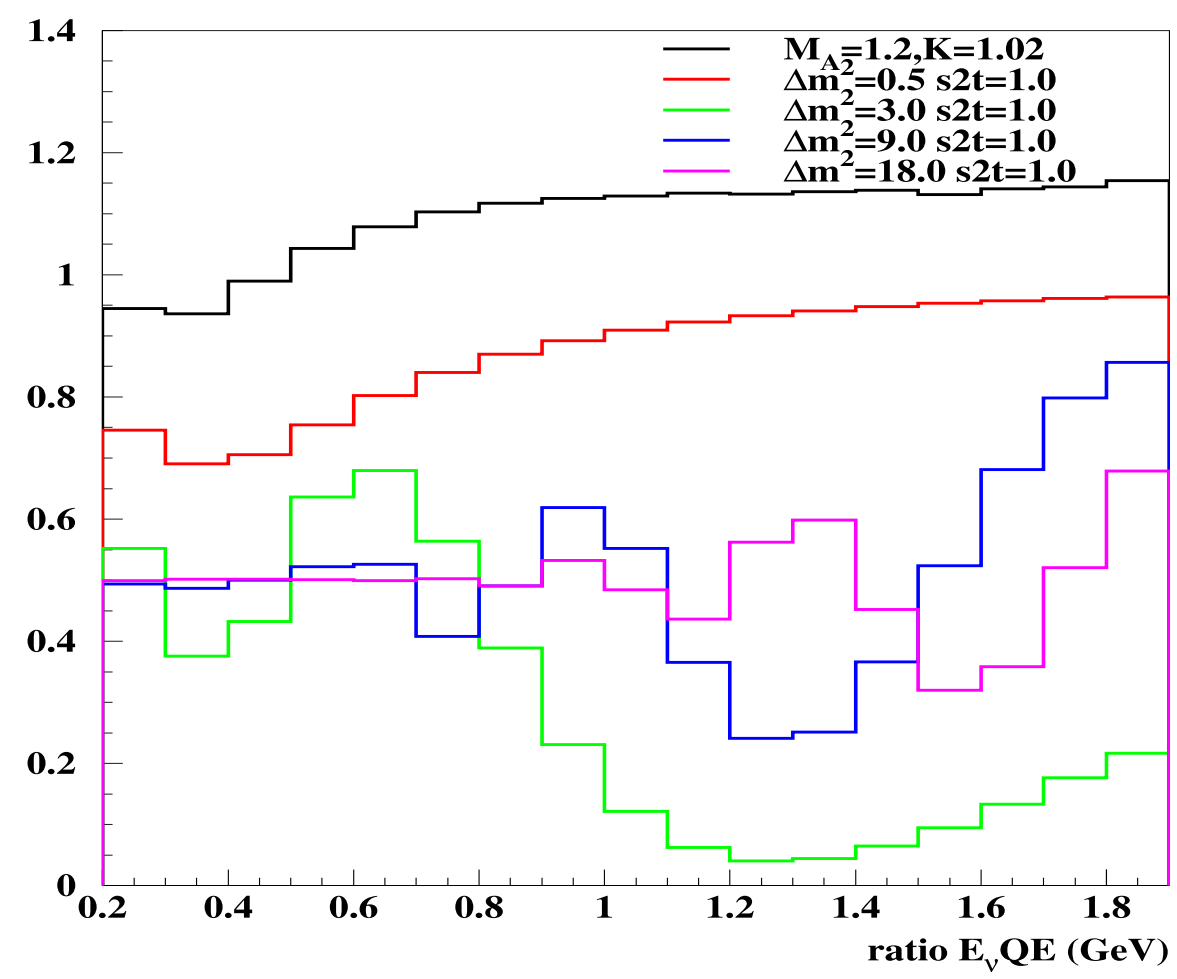

Figure 4.19: Ratio of a prediction $M_{A}=1.23 \mathrm{GeV}, \kappa=1.022$ to $M_{A}=1.00 \mathrm{GeV}$ and $\kappa=$ 1.0000 vs. reconstructed neutrino energy $\left(E_{\nu}^{Q E}\right)$ shown in black for the MiniBooNE CCQE sample. Ratio of prediction assuming oscillation scenarios to the oscillated spectrum with $M_{A}=1.00 \mathrm{GeV}$ and $\kappa=1.0000$ shown in color for $\sin ^{2} \theta=1.0, \Delta m^{2}=0.5 \mathrm{eV}^{2}$ (red), $\Delta m^{2}=3.0 \mathrm{eV}^{2}$ (green), $\Delta m^{2}=9.0 \mathrm{eV}^{2}$ (blue), and $\Delta m^{2}=18.0 \mathrm{eV}^{2}$ (pink). 


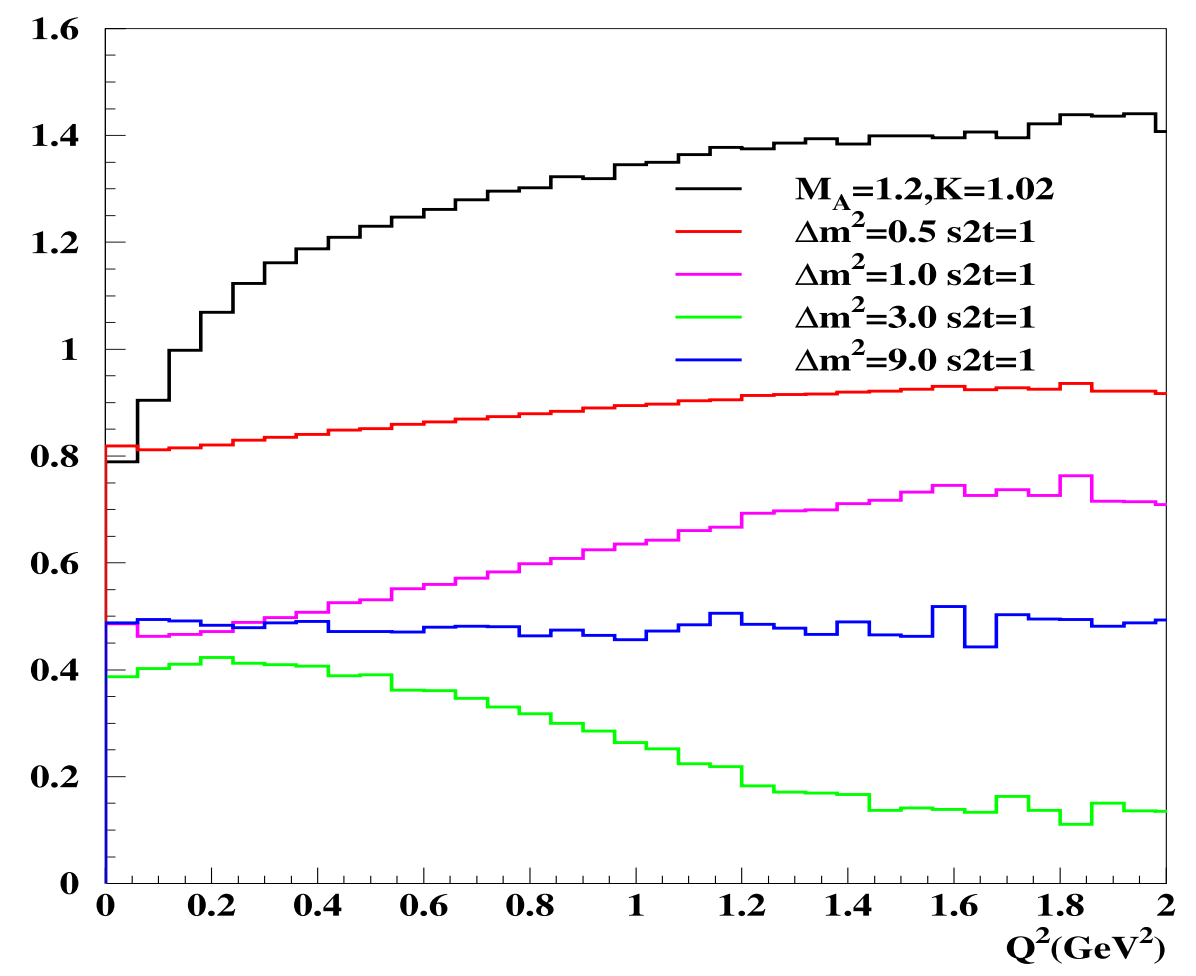

Figure 4.20: Ratio of a prediction $M_{A}=1.23 \mathrm{GeV}, \kappa=1.022$ to $M_{A}=1.00 \mathrm{GeV}$ and $\kappa=1.0000$ vs. reconstructed $Q^{2}\left(\mathrm{GeV}^{2}\right.$ for the MiniBooNE CCQE sample shown in black. Ratio of prediction assuming oscillation scenarios to the unoscillated spectrum with $M_{A}=$ $1.00 \mathrm{GeV}$ and $\kappa=1.0000$ shown in color for $\sin ^{2} \theta=1.0, \Delta m^{2}=0.5 \mathrm{eV}^{2}$ (red), $\Delta m^{2}=$ $3.0 \mathrm{eV}^{2}$ (green), $\Delta m^{2}=9.0 \mathrm{eV}^{2}$ (blue), and $\Delta m^{2}=18.0 \mathrm{eV}^{2}$ (pink) 
The effect on the shape of the neutrino energy spectrum for $M_{A}=1.23, \kappa=1.0220$ is shown in Fig. 4.19. If the cross section model is different, then there is a relative deficit of events at low energy between data and prediction. If there is no change to the cross section model, but instead there is underlying oscillations, the neutrino energy spectrum distorts based on the value of $\Delta m^{2}$. The closest shape distortion to the cross section model corresponds to oscillations at low $\Delta m^{2}=0.5 \mathrm{eV}^{2}$. A change to the cross section model can look similar to oscillation at low energy.

Figure 4.20 shows the effect on the shape of the $Q^{2}$ distribution for both oscillations and changes to the cross section model. The effect of $M_{A}$ and $\kappa$ on $Q^{2}$ is pronounced, as is to be expected since the formalism of the CCQE cross section depends on $Q^{2}$. Oscillations provide relatively little $Q^{2}$ distortion; in particular, oscillations corresponding to $\Delta m^{2}=$ $0.5 \mathrm{eV}^{2}$ result in almost no shape change in $Q^{2}$. Therefore, changing the cross section model will not mask any underlying disappearance in the neutrino or antineutrino mode samples.

For the disappearance analysis, $M_{A}$ is set to the world's data default value on deuterium, $M_{A}=1.015 \mathrm{GeV}, \kappa=1.0000$. The uncertainty on $M_{A}$ and $\kappa$ is set to span the difference between the deuterium and nuclear target results $\left(M_{A}=1.015 \pm 0.23 \mathrm{GeV}\right.$, $\kappa=1.000 \pm 0.019)$. Similarly, for all interactions on hydrogen, $M_{A}=1.015 \pm 0.23 \mathrm{GeV}$. Note that there is no associated $\kappa$ for hydrogen, as there are no nuclear effects on hydrogen. As a result, the cross section uncertainties are slightly lower for antineutrino events.

From Fig. 4.19, the lower energy region is sensitive to the choice of cross section, therefore, large errors on the CCQE cross section will degrade the sensitivity to low $\Delta m^{2} \sim$ $0.5 \mathrm{eV}^{2}$ oscillations as the oscillation effects for low $\Delta m^{2}$ are mainly at low energy. One might improve the sensitivity to disappearance by better constraining the cross section, and such an improved analysis might fit both $Q^{2}$ and $E_{\nu}$ for disappearance. As a near detector constrains both flux and cross section simultaneously, and flux is also a substantial error, the natural improvement to the analysis was to incorporate SciBooNE data as a constraint. 


\subsubsection{CC $\pi$ Production}

Neutrino interactions of sufficient energy can produce pions. There are two channels for this process, resonance production and coherent production of a single pion.

Resonance single pion production occurs when a $\Delta$ is excited and decays:

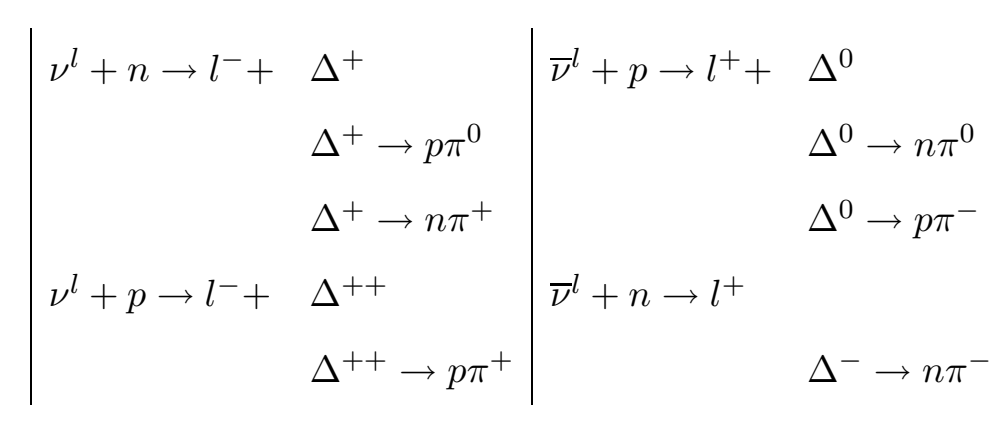

The majority of single pion production is charged pion production at these energies. Resonant production of pions in NUANCE uses the Rein and Seghal formalism [96] which includes the correct pion angular distribution due to the spin structure of the resonances. The dominant resonance at this energy scale is the $\Delta(1232)$ resonance.

Much like the CCQE interaction, a (different) axial form factor $\left(M_{A}^{1 \pi}\right)$ is the single tunable parameter. For CC $\pi^{+}$events, the default is $M_{A}^{1 \pi}=1.10 \pm 0.275 \mathrm{GeV} / c^{2}$ with the uncertainty based on external data of this channel (shown in Fig. 4.16).

$\mathrm{CC} \pi^{+/-}$events are the dominant background in the CCQE sample; if the pion is unobserved, the final state is identical to CCQE events. The MiniBooNE CC $\pi^{+}$data sample indicates a shape difference vs. $Q^{2}$ similar but not identical in nature to the difference observed in the CCQE channel. For the MiniBooNE only analysis, an additional shape error was included to allow the CC $\pi$ background in the CCQE sample to vary in a similar way as observed in the MiniBooNE CC $\pi^{+}$sample. Recent ongoing work indicates that improvements to the underlying model in NUANCE resolve these remaining differences in the MiniBooNE CC $\pi^{+}$sample [97].

Coherent production of pions $\nu^{l}+X \rightarrow l^{-}+\pi^{+}+X^{\prime}$ (or $\bar{\nu}^{l}+X \rightarrow l^{+}+\pi^{-}+X^{\prime}$ ) can also occur when a pion is produced from a coherent scattering of the neutrino off the nuclei. Less than $1 \%$ of all events in the MiniBooNE sample, $4 \%$ of the SciBooNE sample, are expected to be due to coherent production. 


\subsubsection{Final State Internuclear Interactions (FSI)}

Neutrino interactions are modeled as being instantaneous. After the neutrino interaction, any particles produced from the neutrino interaction (e.g. $p, n, \pi)$ can also interact with the nucleus. In NUANCE, the probability for the particle to interact is calculated as the particle is moved in $0.3 \mathrm{fm}$ increments until the particle exits the $2.5 \mathrm{fm}$ radius of the carbon nucleus.

The most important final state interactions for the CCQE analysis occur for CC $\pi^{+/-}$ events:

1. pion absorption $\left(\pi^{+/-}+X \rightarrow X^{\prime}\right)$

2. pion charge exchange $\left(\pi^{+}+X \rightarrow \pi^{0} X^{\prime}\right)$

3. pionless $\Delta$ decay $\left(\Delta^{++} \rightarrow \Delta^{-}, \Delta^{+} \rightarrow \Delta\right)$

as each of these result a final state with no pion. In the first two cases, there is no outgoing pion, so the event looks identical to a CCQE event (CCQE-like). CCQE-like events are an irreducible background as the pion interaction has taken place within nucleus. In the third case, if the photons from the $\pi^{0}$ decay are not identified, the event also appears CCQE-like.

The rate of each of these processes directly affects the CCQE sample in MiniBooNE (Section 5.2.4); increasing pion absorption will increase the number of CCQE-like background events in the CCQE sample as events with a charged pion are eliminated. The SciBooNE sample is CC-inclusive (both CCQE and CC $\pi$ ) and does not depend on a charged pion final state, as discussed in Section 5.1.3, so variations in absorption do not affect the selection.

The default model in NUANCE is shown with the relevant data on pion absorption and charge exchange in Fig. 4.21. Within the nucleus, pion absorption is allowed to vary by $25 \%$, and pion charge exchange $\left(\pi^{+} \rightarrow \pi^{0}\right)$ by $30 \%$. Pionless $\Delta$ decay processes $\left(\Delta^{++} \rightarrow\right.$ $\Delta^{-}, \Delta^{+} \rightarrow \Delta$ ) assume a $100 \%$ uncertainty, and are turned off. 

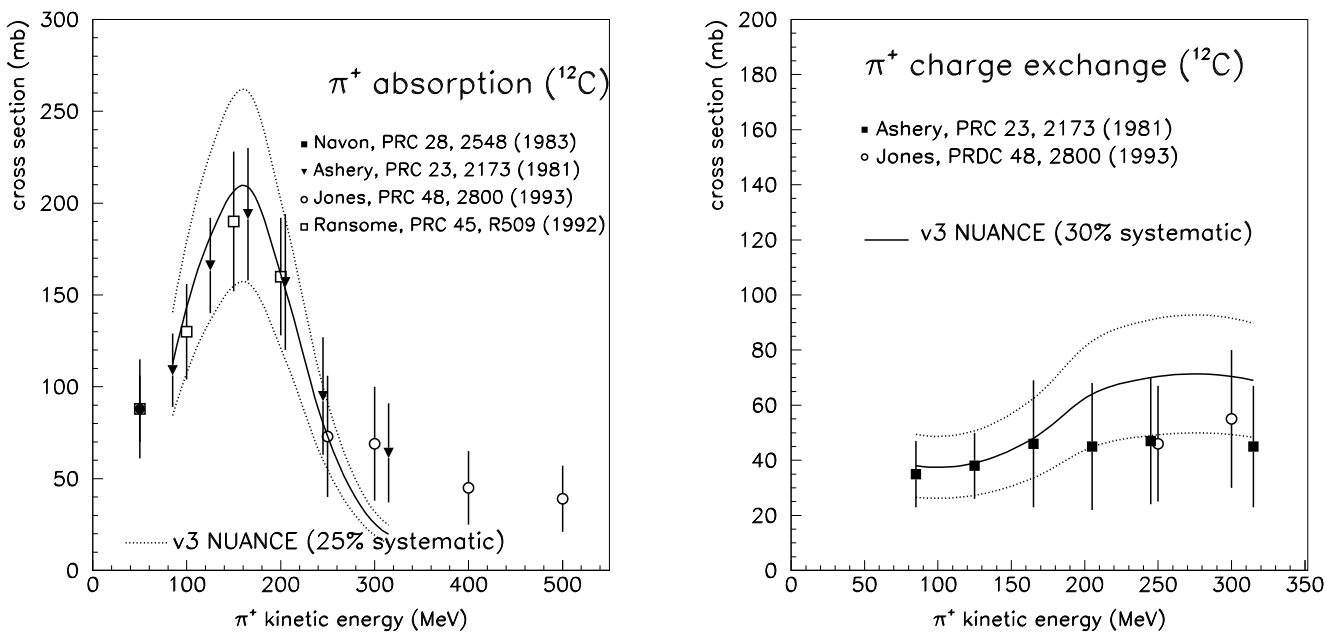

Figure 4.21: Left: Charged pion absorption cross section as a function of pion kinetic energy (MeV). Default cross section (NUANCE) model is shown (black line) with a 25\% systematic (dotted line) and avalible data [98, 99, 100, 101]. Right: Charged pion charge exchange cross section as a function of pion kinetic energy $(\mathrm{MeV})$. Default cross section (NUANCE) model is shown (black line) with a 30\% systematic (dotted line) and avalible data $[99,100]$ 


\subsection{Detector Model}

Though MiniBooNE and SciBooNE share neutrinos, they are inherently different detectors. The simulation of each detector is discussed below.

\subsubsection{SciBooNE Detector Model}

SciBooNE uses a GEANT4 [45] simulation of the detector. SciBar, EC and MRD are modeled with the appropriate material, and in positions based on survey data. The simulation models the experimental hall surrounded by concrete and dirt. The relative position of the SciBar planes to the first layer is set in the simulation based on survey data and cross checked with cosmic ray muon events [35]. The center of the beam axis is modeled as being in the center of SciBar, however, neutrino data indicates a small offset from center $\left(\delta_{x}=-0.11 \mathrm{~cm} \delta_{y}=-3.14 \mathrm{~cm}\right)$ which is included in the positioning of the neutrino interaction.

The detector response in each detector is characterized by data whenever possible. In particular, measurements which serve as input to the simulation of SciBar include:

- Birk's constant (scintillator quenching in the bars follows Birk's law)

- Attenuation length of the WLS fiber

- Crosstalk between MA-PMT channels

- Energy to pe conversion for each channel

The energy loss of a MIP in scintillator is simulated using GEANT4 but tuned using cosmic ray data. Single pe resolution of the MA-PMT, multiple hits and light propagation delays in the WLS are all simulated. In the simulation of the EC, the energy to pe conversion for each channel is based on cosmic ray data, and the attenuation length of the fibers is based on measurement. The MRD simulation includes attenuation in the counters, individual thickness of the iron planes, and small gaps between counters. Figure 4.22 shows the hit efficiency of a typical layer of the MRD; the small drops in efficiency correspond 


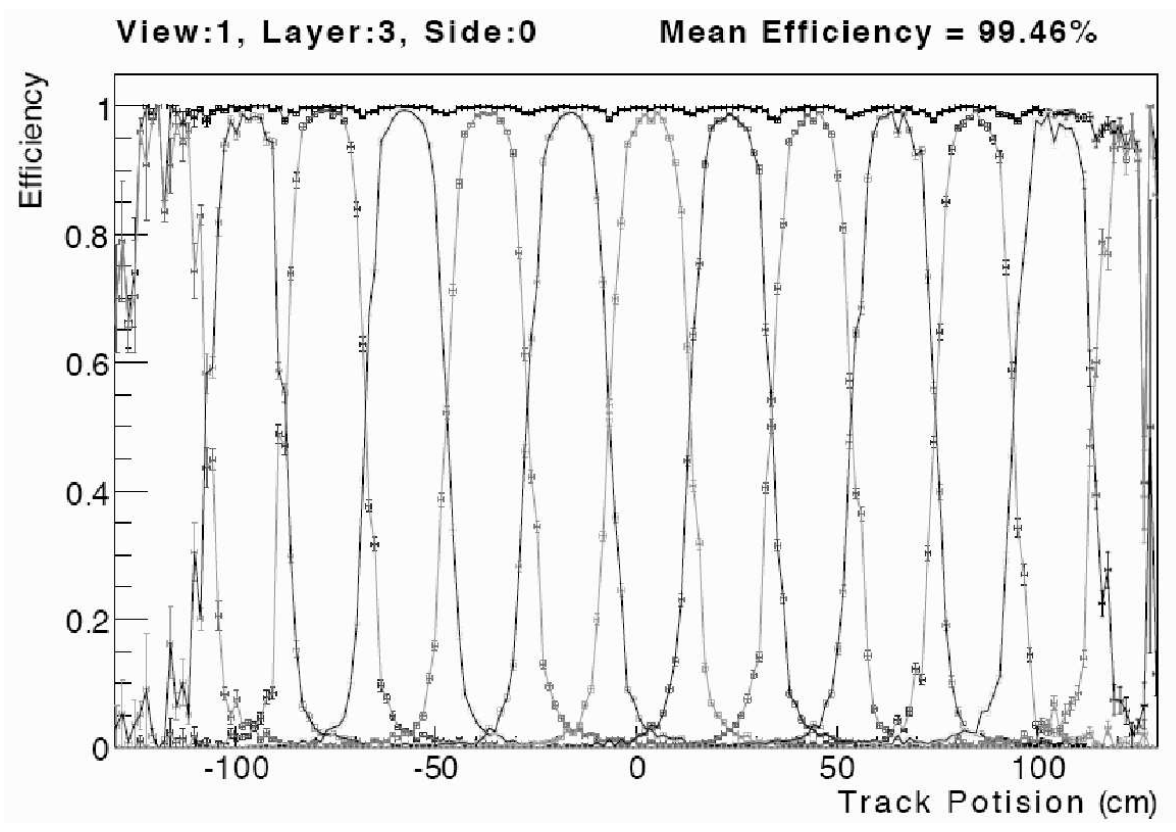

Figure 4.22: Hit efficiency in a typical MRD plane (3rd downstream of EC). The individual counter efficiency (observed hit/expected hit) is shown, along with the total efficiency vs. y position on the plane. The mean efficiency is $99.46 \%$. Slight decreases in the efficiency are due to air gaps between counters.

to gaps between counters. The energy loss in the counters is based on cosmic ray data. Electronics noise and threshold effects are included in the simulation of each detector.

GEANT4 employs the Bertini cascade model to describe hadronic interactions in the detectors. For the SciBooNE detector simulation, this model was scaled to reproduce the data on relevant pion interaction data, as shown in Fig. 4.23. As mentioned later in Section 5.1.3, the SciBooNE selection method does not depend on a pion in the final state, so no additional uncertainty is assumed for this process in SciBooNE.

\subsubsection{MiniBooNE Detector Model}

MiniBooNE uses a GEANT3 [104] simulation of the detector. The detector is assumed to be a sphere with small "top hat" at the top, filled with mineral oil of the appropriate density based on measurement (Section 3.4). The detector sits inside a concrete, cylindrical vault filled with air, which is surrounded by "dirt" $\left(\rho=2.15 \mathrm{~g} / \mathrm{cm}^{3}\right)$ on all sides and 


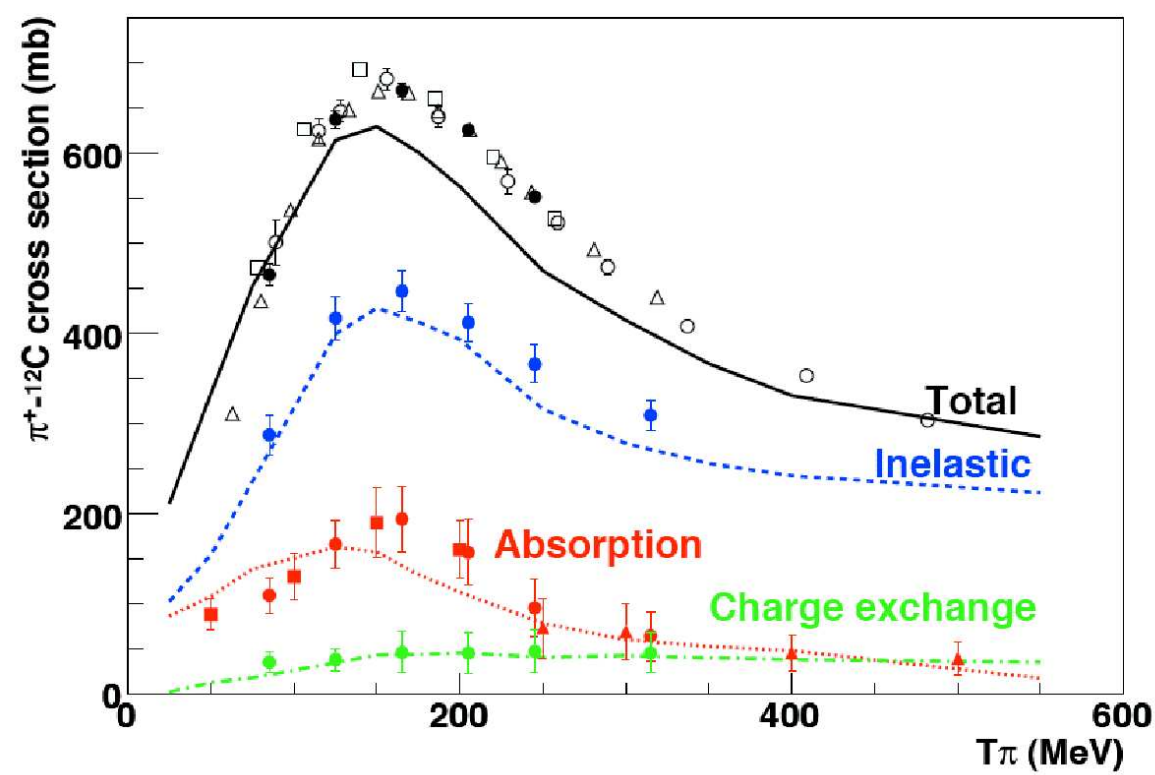

Figure 4.23: Total interaction cross section for pions on carbon vs. the pion kinetic energy $(\mathrm{MeV})$ in the SciBooNE detector model (black line) and available data ( solid o [99], open $\circ$ [102], solid $\square$ [101], open $\square$ [103], solid $\triangle$ [100], open $\triangle$ [50]). The total cross section (black), inelastic (blue), absorption (red) and charge exchange (green) are shown. 

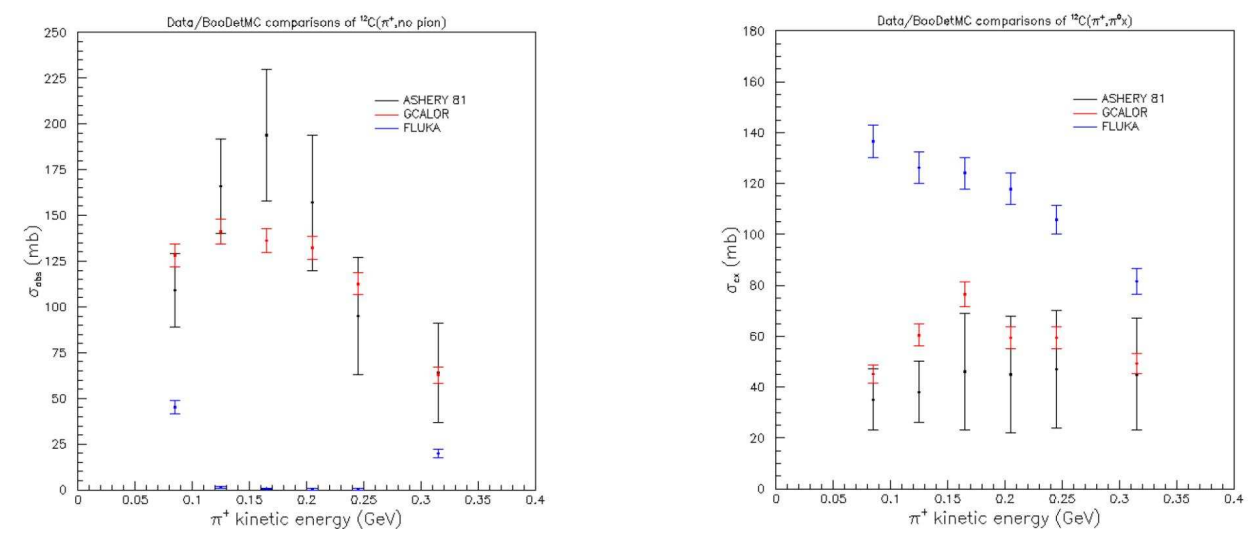

Figure 4.24: Left: Charged pion absorption cross section as a function of pion kinetic energy (MeV). Detector model (GCALOR) model is shown (red points) with available data [99] (black points) and the FLUKA model (blue). Right: Charged pion charge exchange cross section as a function of pion kinetic energy $(\mathrm{MeV})$. Detector model (GCALOR) model is shown (red points) with available data [99] (black points) and the FLUKA model (blue).

above the detector. The scintillator cubes and muon tracker are also simulated. The PMTs are modeled based on their tube type and measured response. Scattering and reflections in the tank and veto are also included.

The GEANT3 default simulation is used to model most particle and light propagation in the tank. Absorption, fluorescence, and Cherenkov light components are all simulated. The two notable exceptions are the muon capture model (custom) and the final state hadronic model (GCALOR [106]). Approximately 7\% of $\mu^{-}$capture on mineral oil. Initially, the default simulation caused $\mu^{-}$to vanish entirely; now the simulation correctly models the low energy photons and neutrons produced upon capture. More information can be found under Ref. [105].

The default hadronic model, FLUKA, has no charged pion absorption in it, as shown in Fig. 4.24. As pion absorption does occur at these energies, the model was changed to GCALOR. The uncertainty on pion absorption in the MiniBooNE model is 35\%, and is $50 \%$ on pion charge exchange. 


\section{Chapter 5}

\section{Event Selection and Reconstruction}

To search for $\nu_{\mu}$ disappearance, we need a sample of pure $\nu_{\mu}$ events where the neutrino energy is known, as oscillations are characterized based on the distortion of the neutrino energy spectrum. The CCQE interaction $\left(\nu_{\mu}+n \rightarrow \mu^{-}+p\right.$ or $\left.\bar{\nu}_{\mu}+p \rightarrow \mu^{+}+n\right)$ is a simple reaction and the most abundant at neutrino energies of a few $\mathrm{GeV}$. The energy and direction of the outgoing muon are sufficient to reconstruct the incoming neutrino energy (Section 5.2.6). Therefore, selecting muons selects $\nu_{\mu}$ and provides energy information about the neutrino.

This section details how neutrino events are selected in SciBooNE and MiniBooNE. After neutrino events are selected, additional cuts create a CC inclusive or a CCQE sample in each detector. The properties of each detector sample are discussed along with the method of reconstructing the incident neutrino energy.

At the end of this section, the two samples are compared to each other in terms of the neutrinos that they measure.

\subsection{SciBooNE}

SciBooNE is a tracking detector; CC interactions will produce a muon which reaches the MRD in most cases, so selecting events which reach the MRD selects $\nu_{\mu}$ and $\bar{\nu}_{\mu}$. First, tracks are reconstructed in SciBar, and then matched to tracks or hits in the MRD. 


\subsubsection{SciBar Tracks}

A hit in SciBar corresponds to more than 2 pe $(0.2 \mathrm{MeV})$ in a scintillator strip. If a hit in the first layer of SciBar is associated with further hits which form a track, the event is rejected. This first layer cut acts as a veto from events produced outside the detector, as SciBooNE has no dedicated veto system.

A two dimensional track (2D) is a set of associated hits in either $x-z$ or $y-z$ planes. If more than three layers are hit $(8 \mathrm{~cm})$, a $2 \mathrm{D}$ track is reconstructible. Hits are combined into a reconstructed track using a cellular automaton algorithm developed on K2K. First, a correction for cross talk is applied to both data and prediction before track reconstruction. Then, remaining hits are categorized into "clusters", where a cluster is one or more hits in adjacent scintillator strips. Segments are formed which connect individual clusters no more than one scintillator strip apart. Any segments which each share a cluster are then also connected, provided the $\chi^{2}$ of a least squares linear fit remains acceptable. More details can be found under Refs. [35, 107]; the efficiency for finding at least one track in a CC event is estimated to be $96 \%$.

Three dimensional tracks are formed from 2D tracks assuming the timing between the two dimensional tracks is within $50 \mathrm{~ns}$, and the start and end point in the $\mathrm{z}$ direction is within $6.6 \mathrm{~cm}$.

\subsubsection{SciBar-MRD Matched Tracks}

The SciBar track is then associated to an MRD track or hits in the MRD. To reconstruct a track in the MRD, at least two layers need to be hit on both the $x$ and $y$ planes. This requires muons of energy greater than $350 \mathrm{MeV}$, as the muon must travel through a minimum of three iron planes. If a track is being matched, the MRD track must originate on the first or second layer of the MRD. The angle of the MRD track with respect to the beam direction, $\theta_{M R D}$, must be consistent with the angle of the SciBar track, $\theta_{S B}$ where $\left|\theta_{M R D}-\theta_{S B}\right|<\theta_{\max }$. The value of $\theta_{\max }$ depends on the length of the MRD track and ranges from 0.4-1.1 radians. Both tracks are extrapolated to the first layer of the MRD, and the difference between the two tracks on the first layer must be $30 \mathrm{~cm}$. Finally, the 
time difference between the SciBar track and the MRD track must be within $100 \mathrm{~ns}$.

If MRD hits are being matched to a SciBar track, the hits in the MRD must be within an cone of \pm 0.5 radians of the end of the SciBar track, and must be no more than $10 \mathrm{~cm}$ away from the end of the SciBar track on the first plane. Timing must also be consistent.

\subsubsection{Beam Quality Selection}

Beam quality cuts are applied to all SciBooNE (and MiniBooNE) data to remove events without:

- sufficient beam intensity $\left(>0.1 \times 10^{11}\right.$ protons per spill)

- agreement between the two proton intensity monitors (Toroid $\_860$ and Toroid $\_75$ within $10 \%$ )

- appropriate horn current for the run mode $\left(>170 \mathrm{kA}\right.$ for $\left.\nu_{\mu}\right)<-170 \mathrm{kA}$ for $\left.\bar{\nu}_{\mu}\right)$

- beam on target (targeting efficiency $>90 \%$ )

This removes less than $2 \%$ of SciBooNE data.

\subsubsection{CC inclusive $\nu_{\mu}$ selection in SciBooNE}

The following cuts select a CC inclusive sample in SciBooNE:

- A SciBar-MRD matched track

- Particle stopped in MRD (no hits in last layer of MRD)

- Particle did not exit the MRD on the side (track endpoint must be contained $|x|<$ $130 \mathrm{~cm}$ and $|y|<110 \mathrm{~cm}$ ).

- Mean time of the track $(t)$ is within the beam time window $(0<t<2 \mu \mathrm{s})$

- SciBar track vertex is within the fiducial volume $(-130 \mathrm{~cm}<x<130 \mathrm{~cm},-130 \mathrm{~cm}<$ $y<130 \mathrm{~cm}, 2.62 \mathrm{~cm}<z<157.2 \mathrm{~cm})$ 
The fiducial volume requirements ensure that the muon energy is correct, and the timing cut ensures that the cosmic contamination is reduced to $0.5 \%$.

The selection cuts are approximately $28 \%$ efficient at selecting CC events within the fiducial volume; the source of the inefficiency stems from acceptance in the detector volume and the effective low energy cut by requiring events to reach the MRD.

According to the prediction, the CC $\nu_{\mu}$ sample is $54 \% \mathrm{CCQE}, 31 \% \mathrm{CC} \pi^{+}$and $6 \% \mathrm{CC} \pi^{0}$ and $2 \%$ deep inelastic scattering (DIS) The remaining $7 \%$ are predominantly multi-pion events. The selection just tags a single muon like particle, and is independant of other final state particles, such as pions, resulting in the 54\% CCQE purity.

The sample is predominantly $\nu_{\mu}$; less than a percent come from $\nu_{e}$ and $1.9 \%$ are $\bar{\nu}_{\mu}$. $90.3 \%$ of the neutrinos in the sample come from $\pi^{+} \rightarrow \mu^{+}+\nu_{\mu}$, and 7.5\% come from $K^{+} \rightarrow \mu^{+}+\nu_{\mu}$. The $p-\theta$ range for neutrino parents is shown in Fig. 5.1.

The energy of the neutrino is reconstructible from only the outgoing muon's energy and angle, assuming the target nucleon is at rest and the interaction was quasi-elastic:

$$
E_{\nu}^{Q E}=\frac{2\left(\left(M_{n}-E_{B}\right) E_{\mu}-\left(E_{B}^{2}-2 M_{n} E_{B}+M_{\mu}^{2}+\left(M_{n}^{2}-M_{p}^{2}\right)\right.\right.}{2\left[\left(M_{n}-E_{B}\right)-E_{\mu}+p_{\mu} \cos \theta_{\mu}\right]}
$$

where $E_{B}$ is the binding energy of the nucleon, $M_{x}$ is the mass of $x$, where $x$ indicates muon, proton or neutron, $E_{\mu}\left(p_{\mu}\right)$ is the reconstructed muon energy (momentum) and $\theta_{\mu}$ is the reconstructed muon scattering angle. The choice of binding energy here is $25 \mathrm{MeV}$; the difference between this and the value used in NUANCE of $34 \mathrm{MeV}$ is negligible.

Figure 5.1.3 shows the $E_{\nu}^{Q E}$ distribution compared to $E_{\nu}$ for events in SciBooNE. While the reconstructed energy is closely related to the true energy for CCQE events, in the case of $\mathrm{CC} \pi^{+}$background events in the neutrino sample, the events are misreconstructed to a lower $E_{\nu}^{Q E}$ as compared to the true value of $E_{\nu}$. This is due to the assumption that all events in the CCQE sample are quasi-elastic and the neutrino energy reconstructed accordingly. The energy resolution for CCQE events is shown in Fig. 5.3; it is approximately $10 \%$ except for events with large true neutrino energy. 


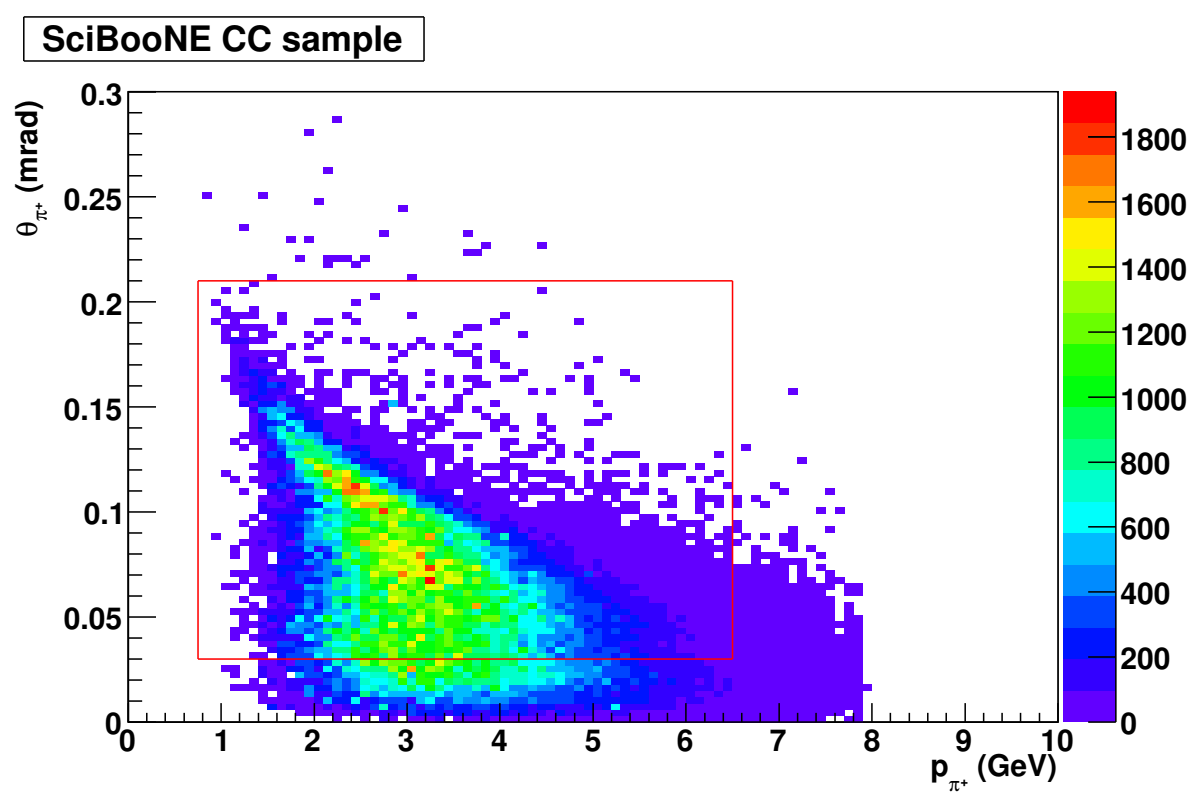

SciBooNE CC sample

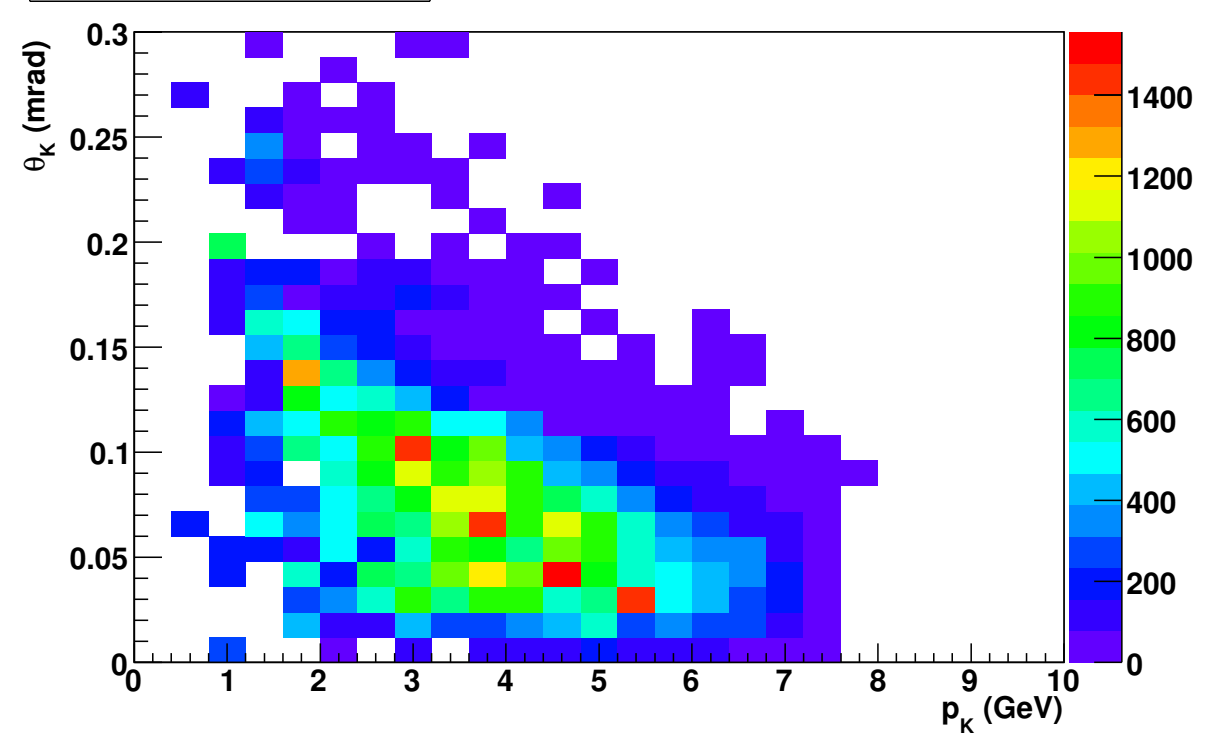

Figure 5.1: 2D histogram of parent momentum $(\mathrm{GeV} / \mathrm{c})$ versus parent angle (rad) for events in the SciBooNE CC neutrino sample. Left: $\pi^{+} \rightarrow \mu^{+}+\nu_{\mu}$ events. The HARP measurement region is shown as a red box. Right: $K^{+} \rightarrow \mu^{+}+\nu_{\mu}$ events. 

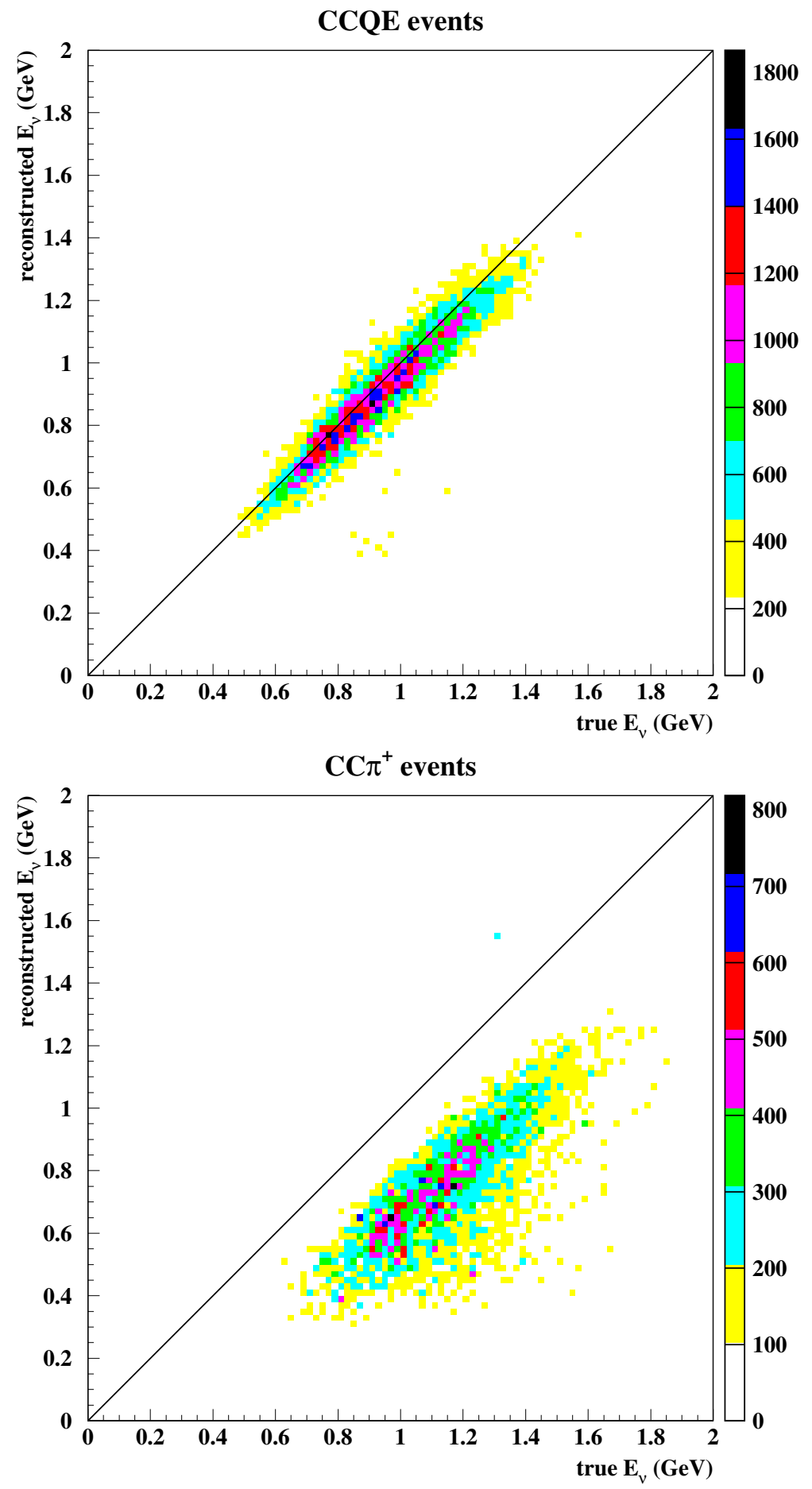

Figure 5.2: $2 \mathrm{D}$ histogram of reconstructed $E_{\nu}^{Q E}$ vs. generated $E_{\nu}$ for CCQE (top) and $\mathrm{CC} \pi^{+}$(bottom) events in SciBooNE; the black line is at $\mathrm{y}=\mathrm{x}$. 


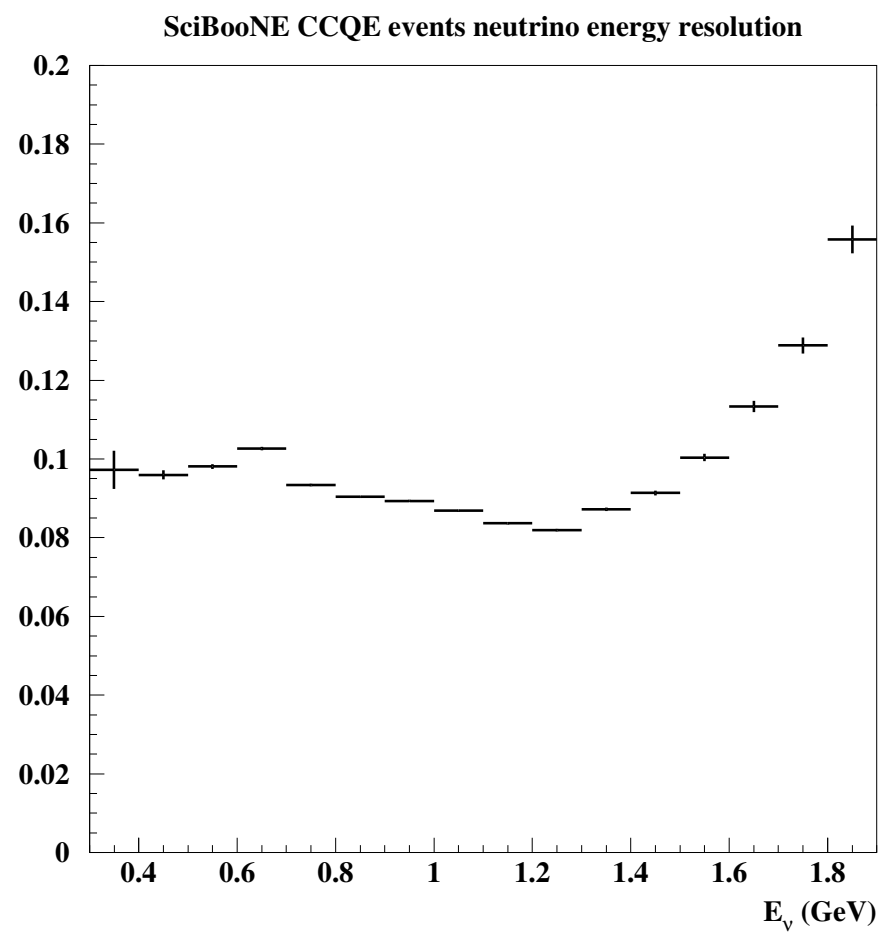

Figure 5.3: Neutrino energy resolution (reconstructed $E_{\nu}^{Q E}$-generated $E_{\nu}$ )/ generated $E_{\nu}$, for CCQE events in the SciBooNE CC inclusive sample as a function of generated $E_{\nu}$ in $100 \mathrm{MeV}$ bins. 

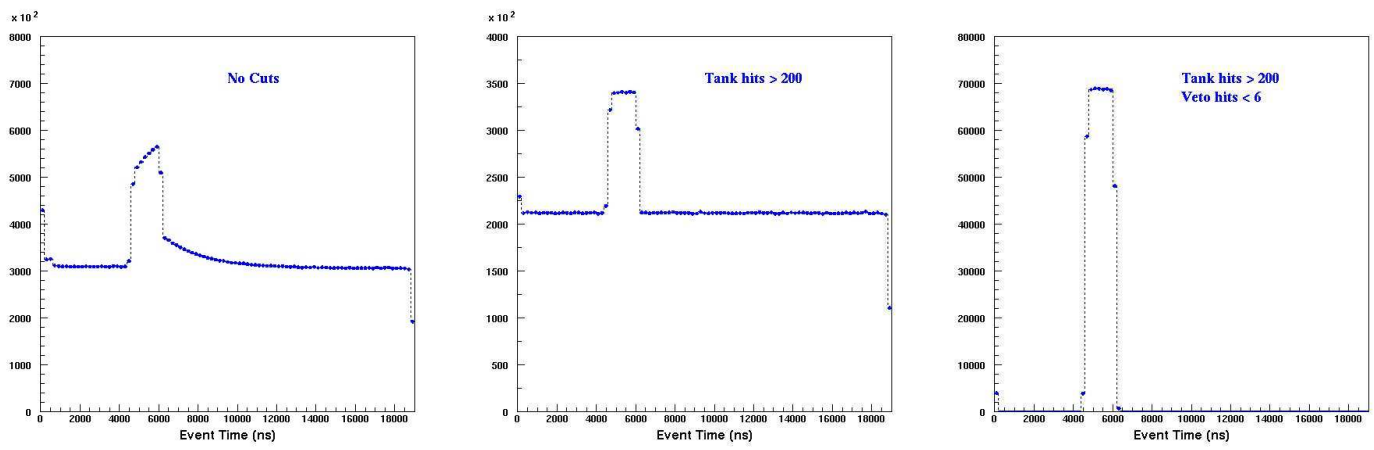

Figure 5.4: Average time (ns) of the first subevent for all events in MiniBooNE. Left: No selection is applied. Middle: A minimal energy threshold is required (main tank hits $>200$ ). Right: Containment is also required with minimal activity in the veto (veto hits $<6)$.

\subsection{MiniBooNE}

\subsubsection{Selection of Beam-Induced Events}

An event is defined on MiniBooNE to be any collection of PMT activity across the entire DAQ time window of $19.2 \mu \mathrm{s}$. Each event can have one or more "subevents", i.e. clusters of hits separated in time. Specifically, more than 10 PMT hits need to occur with no more than $10 \mathrm{~ns}$ between consecutive hits to be categorized as a subevent.

Fig. 5.4 shows the time window for all events in MiniBooNE. Outside the beam window centered on $\sim 5000 \mathrm{~ns}$ is a flat rate of cosmic induced events. The additional exponential decay structure within the beam window and trailing the beam window is caused by electrons from muon decay. To remove the decay electron events, a minimal energy is required, as electrons from muon decay all have energy less than $52.3 \mathrm{MeV}$. By requiring enough tank hits (> 200 main tubes fired) and the exponential structure (and the electrons) vanish. If additionally the events are required to be contained with minimal activity in the veto ( $<6$ veto tubes fired), then rightmost plot in Fig. 5.4 shows a flat distribution of neutrino candidate events in only the beam window.

These "precuts" (main tank hits $<200$ and veto hits $<6$ ) select only neutrino induced 
interactions caused by the beam, and are applied for all further discussions of selection.

\subsubsection{Reconstruction of Muon Events}

To reconstruct events in MiniBooNE, then, one must convert the patterns on the inside of the tank and charge deposited (PMT information) into information about the particle (momentum, position).

Each event is characterized by a hits $q_{i}, t_{i}$ across all PMTs, for some underlying set of parameters $\vec{\alpha}$ which characterize the particle's trajectory. The parameters to uniquely describe a given track in the tank are: position $(x, y, z)$, direction $\left(u_{x}, u_{y}, u_{z}\right)$ and kinetic en$\operatorname{ergy}(\mathrm{E}) ; \vec{\alpha}=\left(x, y, z, u_{x}, u_{y}, u_{z}, E\right)$. The likelihood that we observe $q_{i}, t_{i}$ for the underlying parameters is given by the likelihood function, $L$ :

$$
L=\prod_{i=P M T}^{\text {all }} \prod_{q}^{P M T s} L_{q}\left(q_{i}, \vec{\alpha}\right) L_{t}\left(t_{i}, \vec{\alpha}\right)
$$

where $L_{q(t)}$ is the probability of measuring a charge (time) $q$ at PMT $i$ due to $\vec{\alpha}$. Maximizing the product of the $L_{q}$ and $L_{t}$ gives us the optimal parameters for the event.

The simplest model assumes a point-like source of light in the tank. The reconstructed $\vec{\alpha}$ is calculated in a three step process. First, the charge averaged position $R$ and charged averaged time $T$ of the event is calculated from the time and position of the PMTs $\left(t_{i}, r_{i}\right)$ which registered hits:

$$
\begin{aligned}
& R=\frac{1}{Q} \sum_{i}^{\text {all hits }} q_{i} r_{i} \\
& Q=\sum_{i}^{\text {all hits }} q_{i} \\
& T=\frac{1}{Q} \sum_{i}^{\text {all hits }} q_{i}\left(t_{i}-\left|r_{i}-R\right|\right) n / c
\end{aligned}
$$

Second, a simple time likelihood is formed assuming a Gaussian Cherenkov component and scintillation component with a exponential decay. This is used to calculate a first estimate the direction, $\vec{U}$, of the event: 


$$
\vec{U}=\frac{1}{Q} \sum_{i}^{\text {prompthits }} q_{i}\left(\overrightarrow{r_{i}}-\vec{R}\right)
$$

and the energy, $E=Q / F(r)$, where $F(r)$ is an empirical function based on calibration data.

The third step uses the initial seeds for direction and energy, and uses a time and charge likelihood together to produce an estimate of $\vec{\alpha}$. For the time likelihood, Cherenkov light is modeled with an energy dependent angular distribution, the quantum efficiency of the tubes and the total charge expected given the solid angle seen by the tube are all included. The charge likelihood (probability that a tube sees a charge given an underlying true charge) is formed from the prediction using inputs from calibration data. More details can be found under Refs.[111, 112, 113].

Fig. 5.5 compares true muon variables to reconstructed ones in a series of profile histograms. While the simple point source model is not strictly true for an extended source of light, like a muon, this reconstruction method models muons acceptably and behaves similarly for data and prediction. This method has since been improved[108], but is not used for the work in this thesis.

\subsubsection{Data Quality Selection and Time Dependent Effects}

For all reconstruction in MiniBooNE, any PMTs which fail over the course of the data run are not used for any reconstruction during reprocessing for all time periods. If a tube was live earlier in the run and failed later, it would not be used for reconstruction even for data taken in the earlier time. This is to ensure that cuts on tank hits and reconstructed quantities do not change with time.

The same beam quality cuts (Section 5.1.2.1) are applied to MiniBooNE as SciBooNE, and remove the same fraction of POT. In addition, a list of "bad" runs was compiled by looking at the log in the control room. Runs deemed suspect by people taking MiniBooNE shift were removed from the data stream. This removes another $2 \%$ of data over the initial MiniBooNE data taking run. 

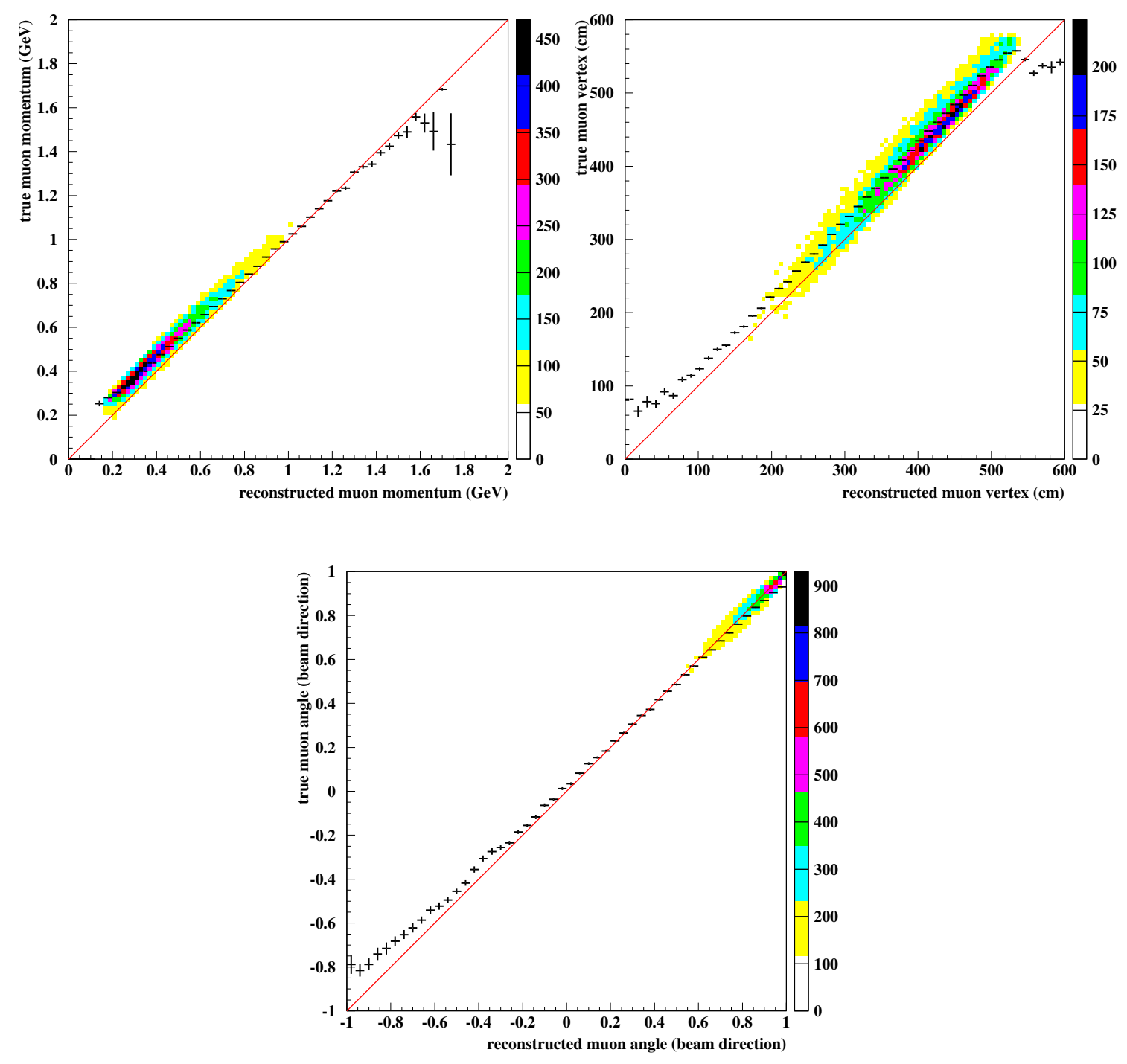

Figure 5.5: Reconstructed versus true muon variables. The black crosses indicate the spread of the distribution (profile histogram) while the color scale is a 2D plot. Left: Muon momentum (GeV) Middle: Distance from center of the tank to the vertex (radius) Right: $\cos \left(\theta_{\text {beam }}\right)$, the angle of the muon with respect to the beam direction. 


\subsubsection{CCQE $\nu_{\mu}$ selection in MiniBooNE}

An unique handle to select CCQE events in MiniBooNE is the number of subevents. If the muon from the neutrino interaction decays to an electron, this produces two separate subevents in the detector, one prompt subevent consistent with a muon, and one delayed subevent consistent with an electron. A CC $\pi^{+}$event will produce three subevents:the muon, the muon's decay electron, and the pion's decay muon's decay electron.

The CCQE event sample is selected by identifying a single muon in the detector and its associated decay electron, with the same criteria as were used for the measurement of quasi-elastic scattering on carbon [94]. Exactly two subevents are required (the muon and the decay electron). To reject cosmic ray interactions, both subevents are required to have less than 6 veto-PMT hits. The first subevent must be in coincidence with a beam pulse, have a reconstructed track center less than $500 \mathrm{~cm}$, and greater than 200 inner tank PMT hits to eliminate electrons from cosmic ray muon decays. The second subevent must have less than 200 inner PMT hits to be below the decay electron energy endpoint.

The last selection is a check that the second subevent (electron) is associated with the first (muon). The distance between the electron vertex and the muon track endpoint must be less than $100 \mathrm{~cm}$, ensuring that the electron decayed from the muon.. The muon track endpoint is calculated assuming the first subevent is a MIP, and using the energy and

direction to calculate the endpoint. This cut preferentially eliminates $\mathrm{CC} \pi^{+}$events, as the decay electron from the pion can be far from the muon's endpoint.

The selection cuts are $35 \%$ efficient within the fiducial volume of $500 \mathrm{~cm}$, which is the product of:

- $50 \%$ probability to contain (muon) events within the inner tank.

- $83 \%$ probability of tagging a muon with a decay electron $\left(\sim 7 \%\right.$ of $\mu^{-}$capture on carbon[114])

- $85 \%$ probability that the electron of the second subevent is close to the endpoint of the muon (first subevent)

According to the prediction, the $\nu_{\mu}$ sample is $74 \% \mathrm{CCQE}, 21 \% \mathrm{CC} \pi^{+}$and $4 \% \mathrm{CC} \pi^{0}$. 


\begin{tabular}{|c|c|c|c|c|c|}
\hline parent & $\pi^{+}$ & $\pi^{-}$ & $K^{+}$ & $K^{0}$ & $K^{-}$ \\
\hline fraction of total (\%) & 22.6 & 71.4 & 2.2 & 0.2 & 0.2 \\
\hline
\end{tabular}

Table 5.1: Meson parents of neutrinos or antineutrinos in the CCQE sample, fractionally, for antineutrino running

The remaining $1 \%$ are multi-pion events or neutral current pion production events.

The primary background $(\sim 75 \%)$ to the CCQE selection are $\mathrm{CC} \pi^{+}$events. A CC $\pi$ event in MiniBooNE produces three subevents: primary muon, primary muon decay electron, and decay electron from pion decay. Selecting on two subevents naturally removes many $C C \pi$ events in MiniBooNE. For a CC $\pi$ to become background, the outgoing pion must be unobserved, and thus produce no third subevent. This can happen when the pion is absorbed in the nucleus, decays along the muon track, or when the pion's decay electron does not produce enough hits to create a third subevent.

Almost all (99\%) of the events in the neutrino sample come from $\pi^{+} \rightarrow \mu^{+}+\nu_{\mu}$, and the remainder are from $K^{+} \rightarrow \mu^{+}+\nu_{\mu} .88 \%$ of the sample is contained within HARP's measurement region $\left(0.75<p_{\pi}<6.5 \mathrm{GeV}, 0.03<\theta_{\pi}<0.210 \mathrm{mrad}\right)$, and $99 \%$ is contained within HARP and low pion angles $\left(\theta_{\pi}<0.03 \mathrm{mrad}\right)$, as shown in Fig. 5.6.

\subsection{5 $\operatorname{CCQE} \bar{\nu}_{\mu}$ Selection}

All selection cuts are applied in the exact same way for antineutrino data, because the final state nucleon is not observed, and the detector does not distinguish muon charge.

For antineutrino mode, the beam is not pure $\bar{\nu}_{\mu}$ but contains a substantial contribution of $\nu_{\mu}\left(25 \%\right.$ of the sample) due to the higher $\pi^{+}$production at the target and the higher $\nu_{\mu}$ cross section. According to the simulation, the antineutrino sample is $69.8 \%$ CCQE, $5.5 \% \mathrm{CC} \pi^{+}, 16.6 \% \mathrm{CC} \pi^{-}$, with the remainder being multi-pion channels. Parentage of the sample is shown in Table 5.1. The momentum, angle of the parent $\pi^{+}$and $\pi^{-}$are shown in Fig. 5.7; while the antineutrinos come from $\pi^{-}$similar to the $\pi^{+}$in neutrino mode (Fig. 5.6), the neutrino contamination is almost entirely due to events which are not defocused by the horn (small angles), and sits outside the HARP measurement region. 


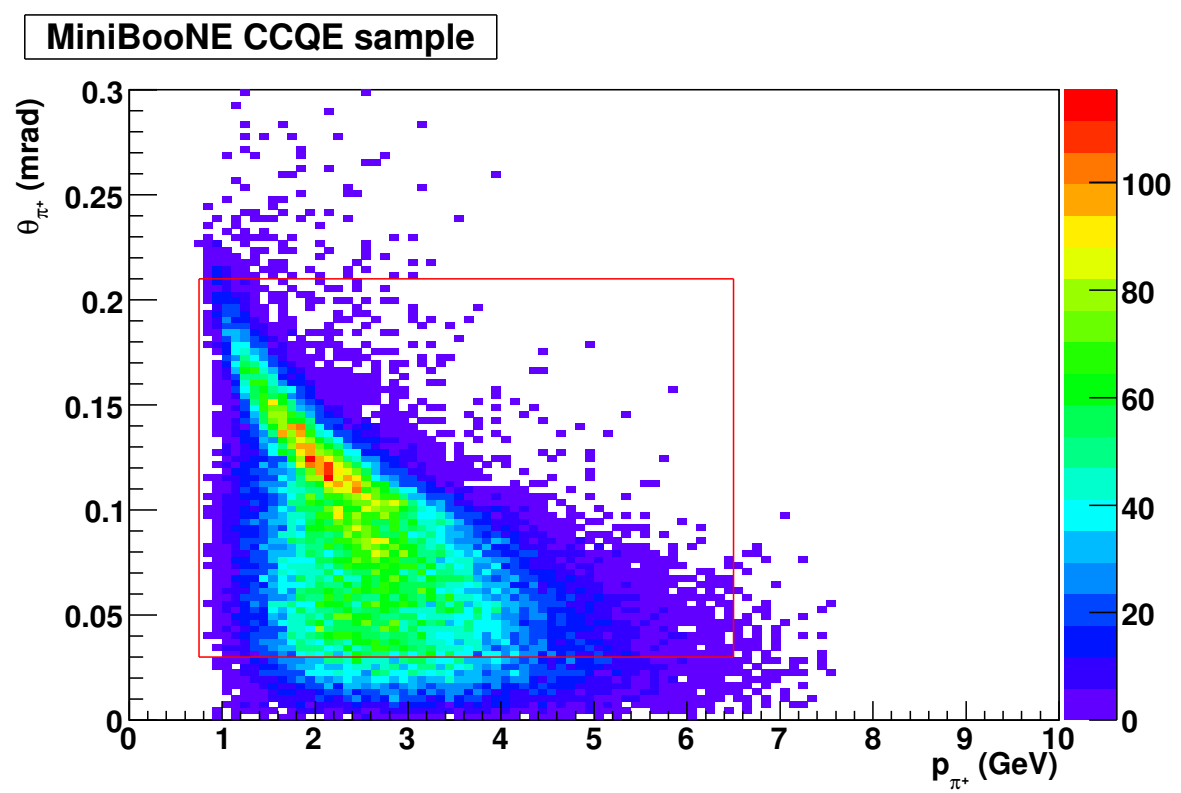

\section{MiniBooNE CC sample}

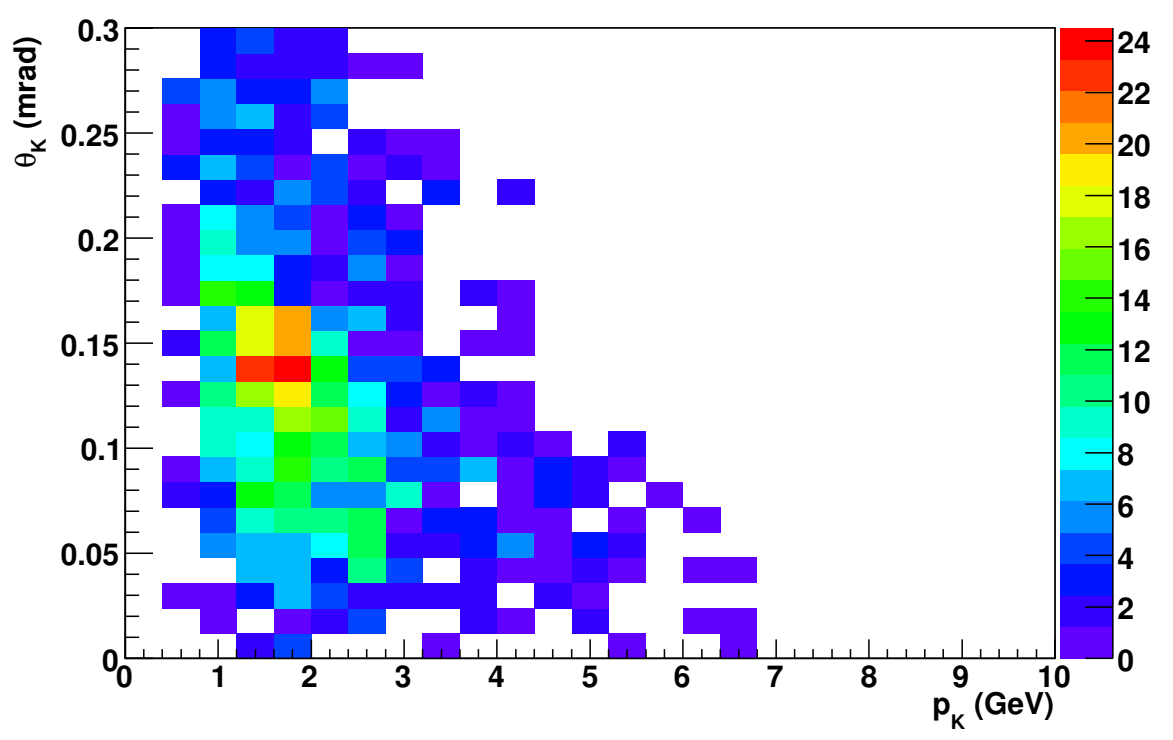

Figure 5.6: 2D histogram of parent momentum $(\mathrm{GeV})$ versus parent angle (rad) for events in the MiniBooNE CCQE neutrino sample. Left: $\pi^{+} \rightarrow \mu^{+}+\nu_{\mu}$ events. The HARP measurement region is shown as a red box. Right: $K^{+} \rightarrow \mu^{+}+\nu_{\mu}$ events. 

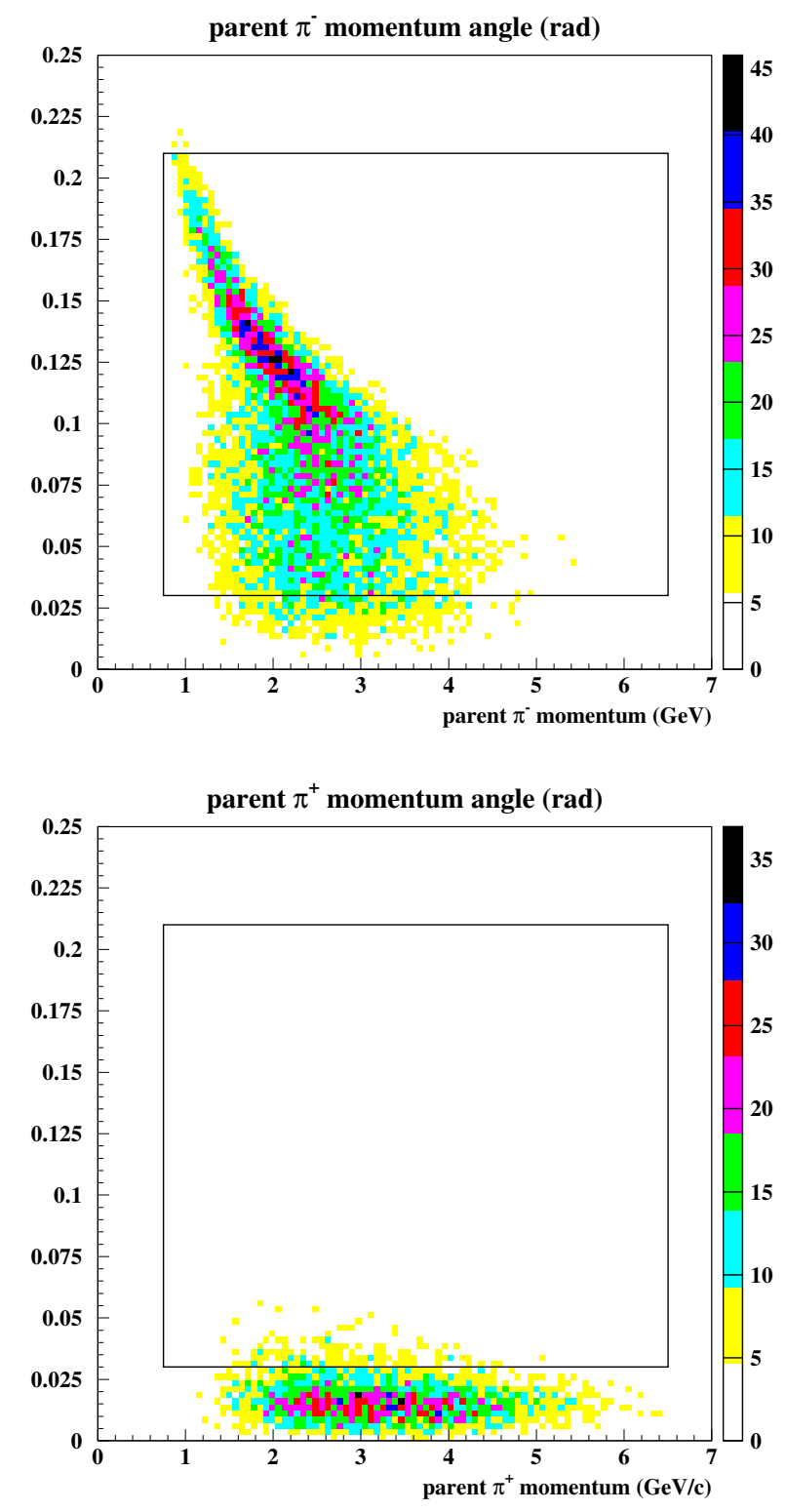

Figure 5.7: 2D histogram of parent momentum $(\mathrm{GeV})$ versus parent angle (rad) for events in the MiniBooNE CCQE neutrino sample. Left: $\pi^{-} \rightarrow \mu^{-}+\bar{\nu}_{\mu}$ events. The HARP measurement region is shown as a red box. Right: $\pi^{+} \rightarrow \mu^{+}+\nu_{\mu}$ events. The HARP measurement region is shown as a red box. 


\subsubsection{Neutrino Energy Calculation}

This section draws heavily from Refs. [109] and [110].

Equation 5.1 is also used to calculate the reconstructed neutrino energy on MiniBooNE. An additional set of corrections is applied in both data and prediction samples to create a better estimate of the neutrino energy. There are three corrections, further discussed in Appendix A:

1. A correction as a function of the muon energy.

2. A correction to reconstructed neutrino energy as a function of $Q^{2}$.

3. A final correction as a function of the reconstructed neutrino energy.

Fig. 5.2.6 shows the corrected $E_{\nu}^{Q E}$ distribution as compared to $E_{\nu}$ for events in the MiniBooNE sample. Like SciBooNE, the CCQE events reconstruct $E_{\nu}^{Q E}$ close to $E_{\nu}$, but $\mathrm{CC} \pi^{+}$background events reconstruct at the wrong value of $E_{\nu}^{Q E}$ due to the assumption that they are quasi-elastic.

What is relevant for the disappearance analysis are any data/prediction differences which could mask or create an oscillation signal. This correction is applied for both data and prediction. If we compare the "original" $E_{\nu}^{Q E}$, calculated using Eq. 5.1 with uncorrected muon energy, to the final $E_{\nu}^{Q E}$ with all corrections applied, we see only differences which are covered by the size of the detector prediction uncertainties, discussed in Section 6.3.3. Fig. 5.9 shows the ratio of data to prediction for the two different calculations of neutrino energy. The remaining difference is comparable to the size of the detector systematics, as discussed in Section 7.1, which vary between 2 and 5\% across all energies. As a result any fits using the uncorrected and corrected energy distributions are consistent with each other.

The energy resolution is shown in Fig. 5.10 as a function of true neutrino energy for CCQE events in the MiniBooNE sample.

As the selection is the same for neutrinos as antineutrinos, the $E_{\nu}^{Q E}$ calculation is also the same (this ignores the small $(\sim 1.3 \mathrm{MeV})$ proton to neutron mass difference). 

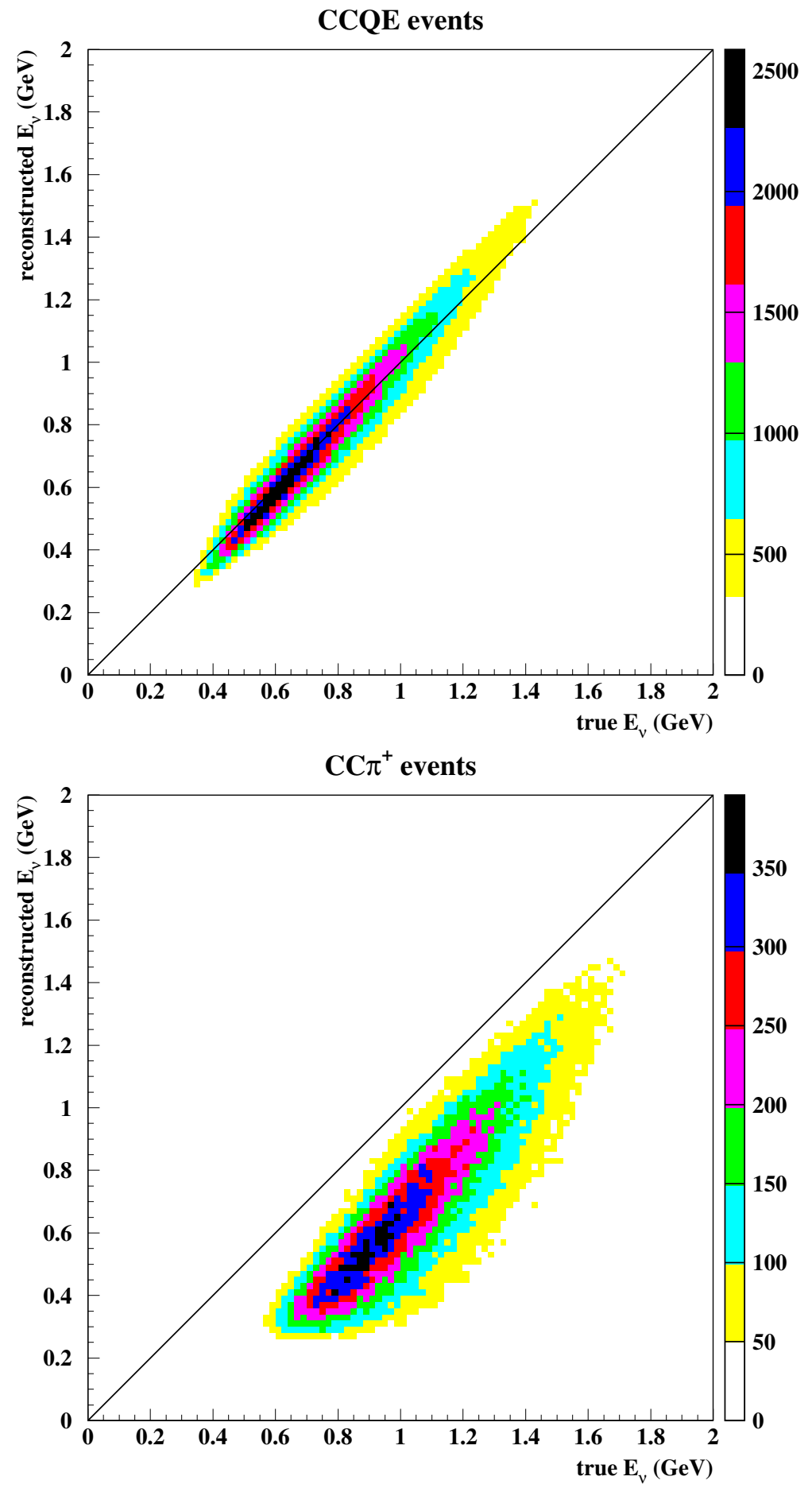

Figure 5.8: $2 \mathrm{D}$ histogram of reconstructed $E_{\nu}^{Q E}$ vs. generated $E_{\nu}$ for CCQE (top) and $\mathrm{CC} \pi^{+}$(bottom) events in MiniBooNE; the black line is at $\mathrm{y}=\mathrm{x}$. 


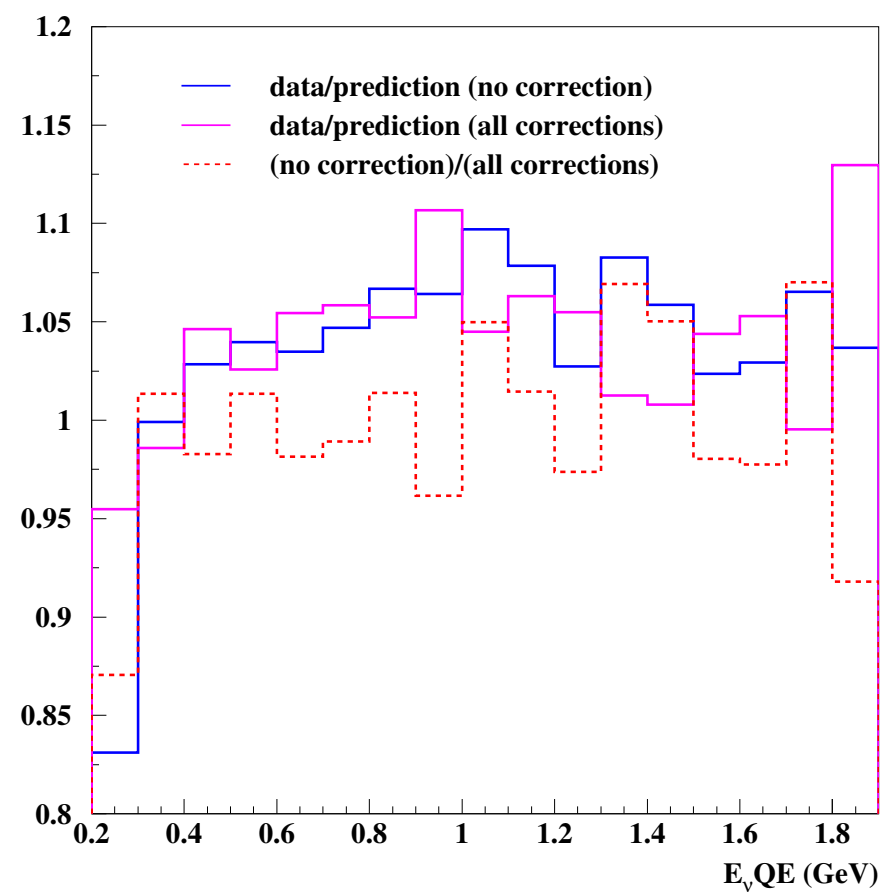

Figure 5.9: Ratio of data to prediction vs reconstructed $E_{\nu}^{Q E}$ calculated with no corrections (blue) and using all corrections (purple). The double ratio of data/prediction for no corrections to all corrections is also shown (red dash). 


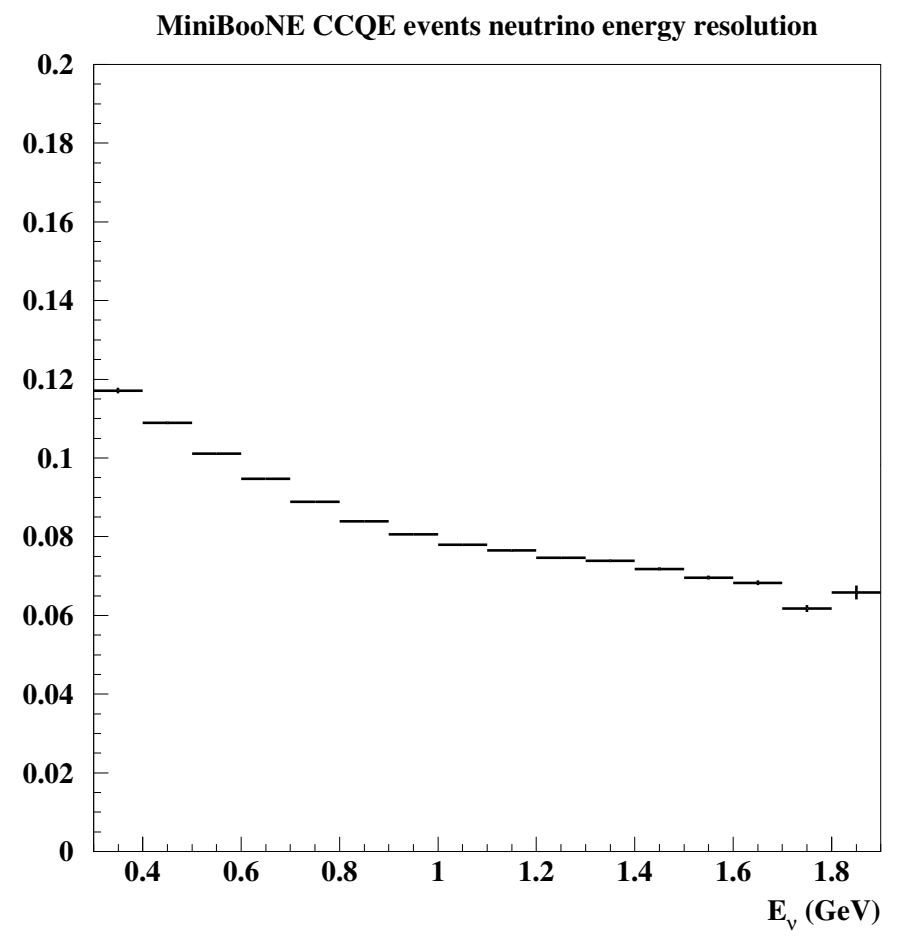

Figure 5.10: Neutrino energy resolution (reconstructed $E_{\nu}^{Q E}$-generated $E_{\nu}$ )/ generated $E_{\nu}$, for CCQE events in the MiniBooNE CCQE sample, as a function of generated $E_{\nu}$ in $100 \mathrm{MeV}$ bins. 
Figure 5.2.6 shows the corrected $E_{\nu}^{Q E}$ distribution compared to $E_{\nu}$ for events in the MiniBooNE antineutrino sample.

\subsection{Comparison between MiniBooNE and SciBooNE samples}

The SciBooNE sample covers the same $\pi^{+}$kinematic region but populates it differently. Figure 5.12 show SciBooNE and MiniBooNE neutrino parent pion kinematic regions, normalized to the same $\left(1 \times 10^{20}\right)$ POT. While the SciBooNE sample covers the entire $\pi^{+}$momentum region of MiniBooNE, the MRD requirement increases the mean energy of the parent $\pi^{+}$substantially. SciBooNE also covers a smaller range in angles; the lowest momentum pions have larger angles and so do not enter into the SciBooNE sam-

ple. Figure 5.13 shows the SciBooNE and MiniBooNE neutrino parent kaon kinematic regions, normalized to the same (1e20) POT. The SciBooNE sample has approximately twice the kaon content as the MiniBooNE sample, and covers a broader range in kaon momentum, despite the MRD containment requirement.

The relative fraction of CCQE-CC $\pi^{+}$events are different between SciBooNE and MiniBooNE, but the coverage in $q^{2}$ is similar between the two samples for CCQE and CC $\pi^{+}$ events, as shown in Fig. 5.14.

Figure 5.15 shows the overlap in generated neutrino energy for the two samples. The behavior in neutrino energy follows the behavior seen in parent pion momentum (Fig. 5.12), the addition of a SciBooNE sample using events which do not reach the MRD would populate the lower neutrino energy region. 

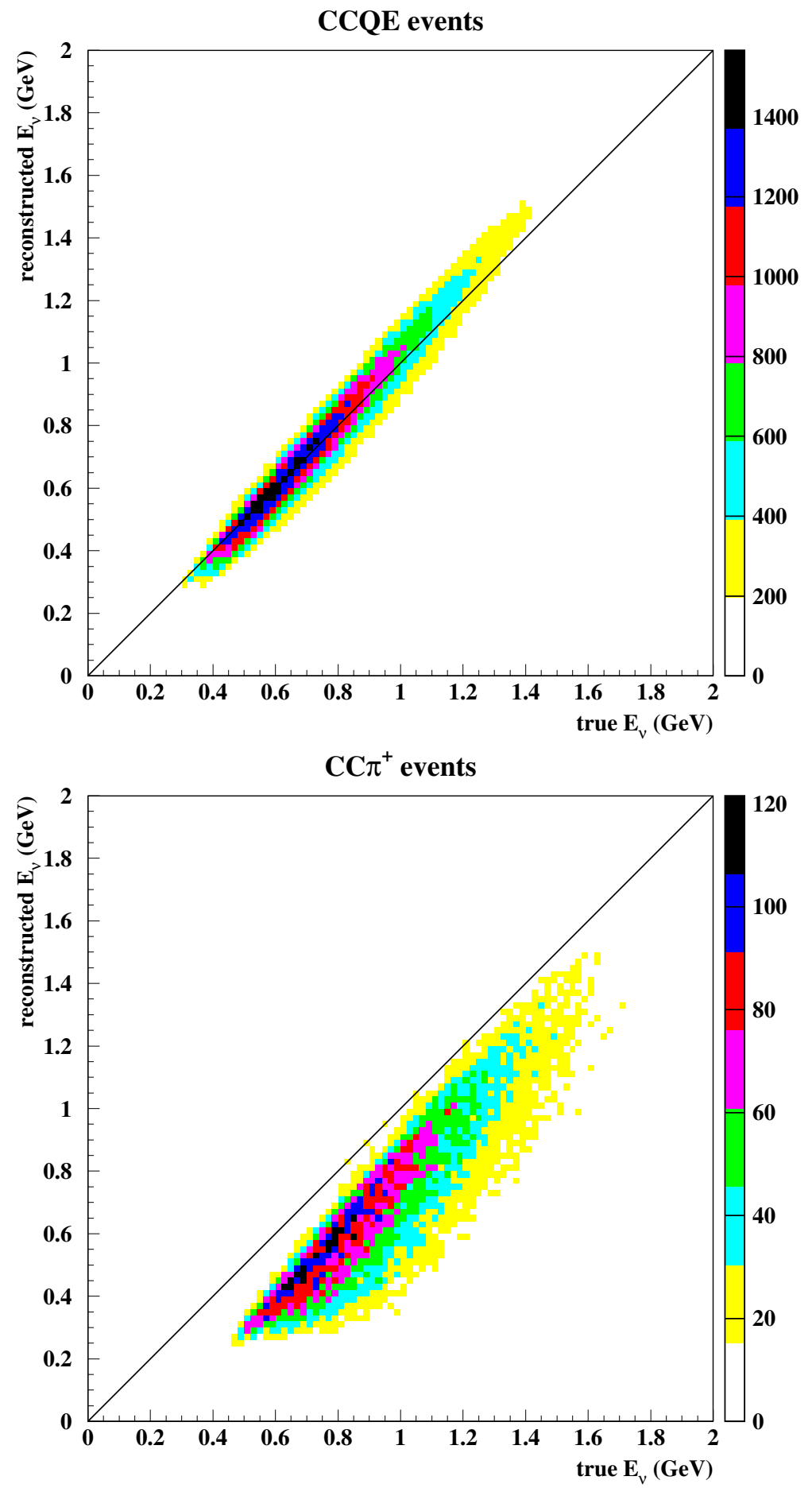

Figure 5.11: 2D histogram of reconstructed $E_{\nu}^{Q E}$ vs. generated $E_{\nu}$ for CCQE (top) and $\mathrm{CC} \pi^{+}$(bottom) events in the MiniBooNE antineutrino sample; the black line is at $\mathrm{y}=\mathrm{x}$. 

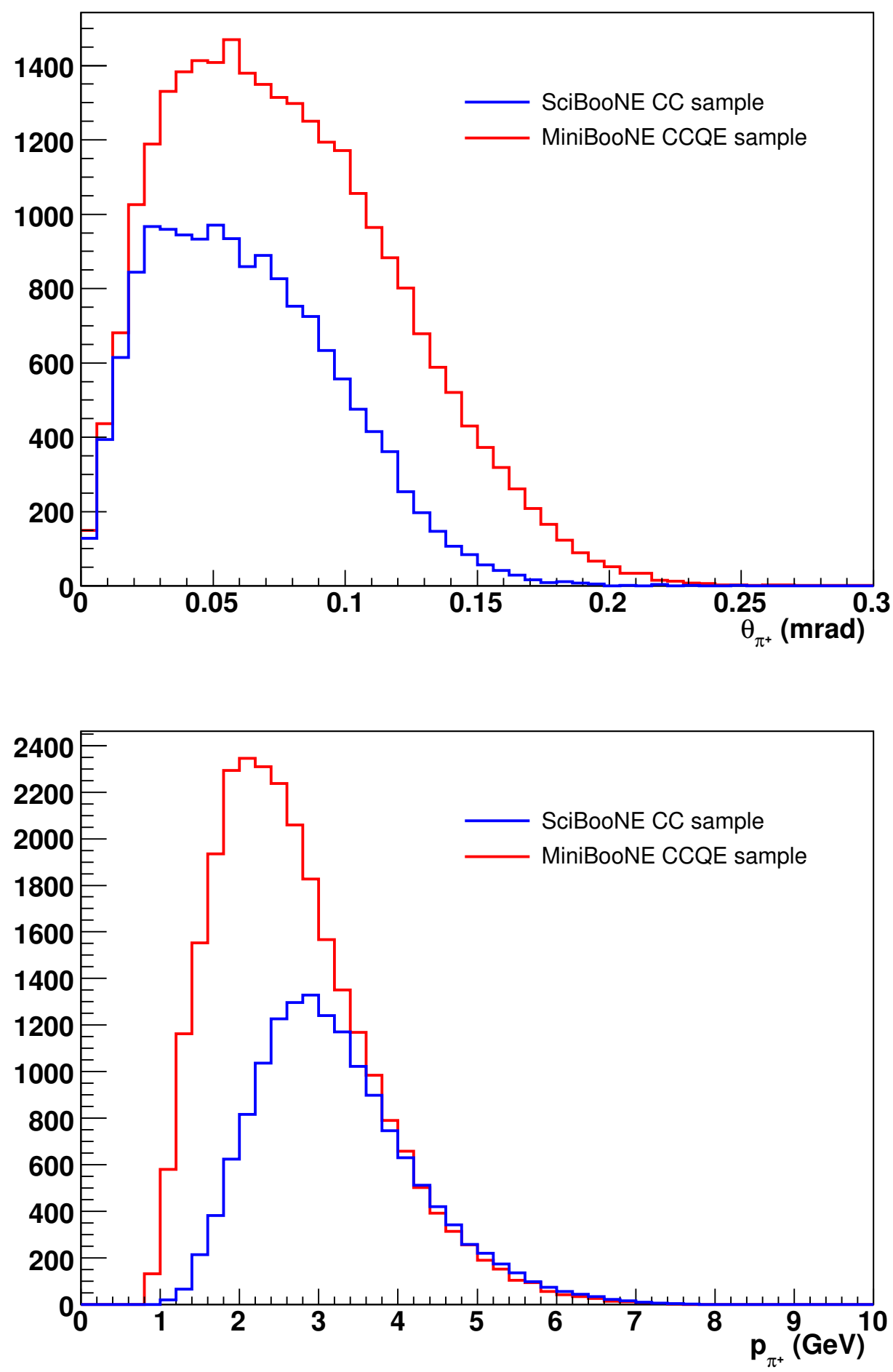

Figure 5.12: Pion parent momentum (left) and angle (right) in the MiniBooNE CCQE sample (red) with the SciBooNE CC sample (blue). The two samples are normalized to equal POT. 

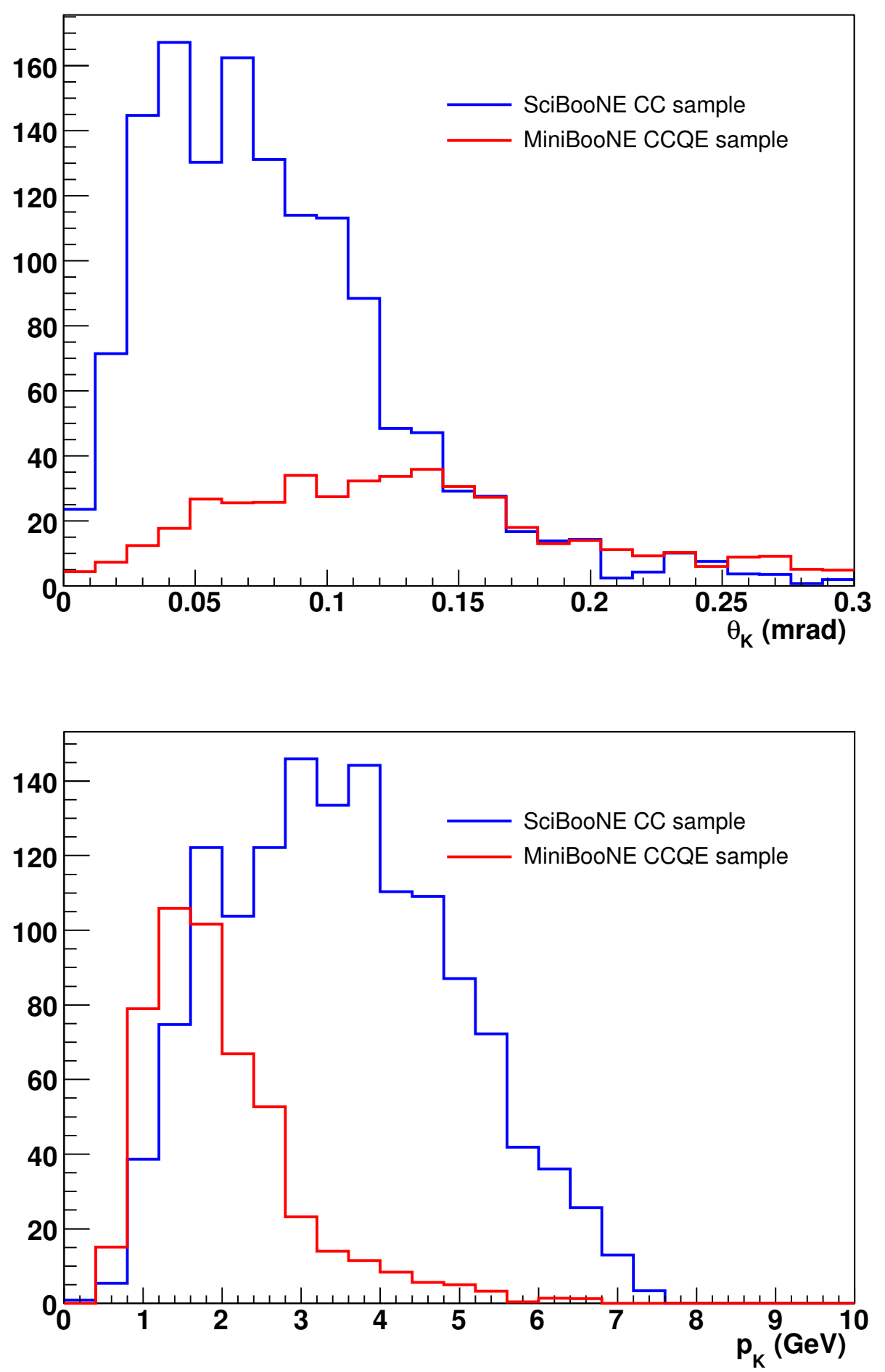

Figure 5.13: Kaon parent momentum (left) and angle (right) in the MiniBooNE CCQE sample (red) with the SciBooNE CC sample (blue). The two samples are normalized to equal POT. 


\section{CCQE events}

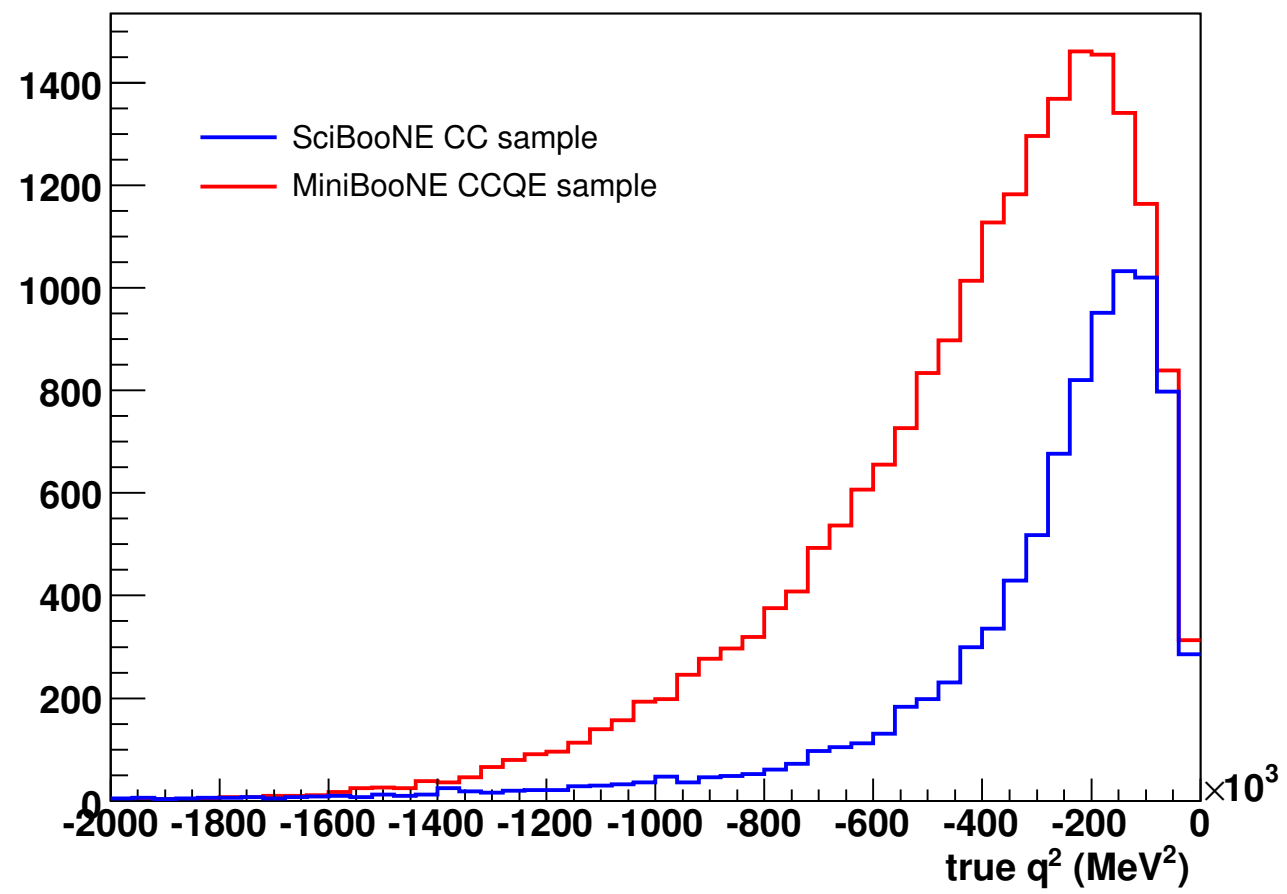

\section{CC $\pi^{+}$events}

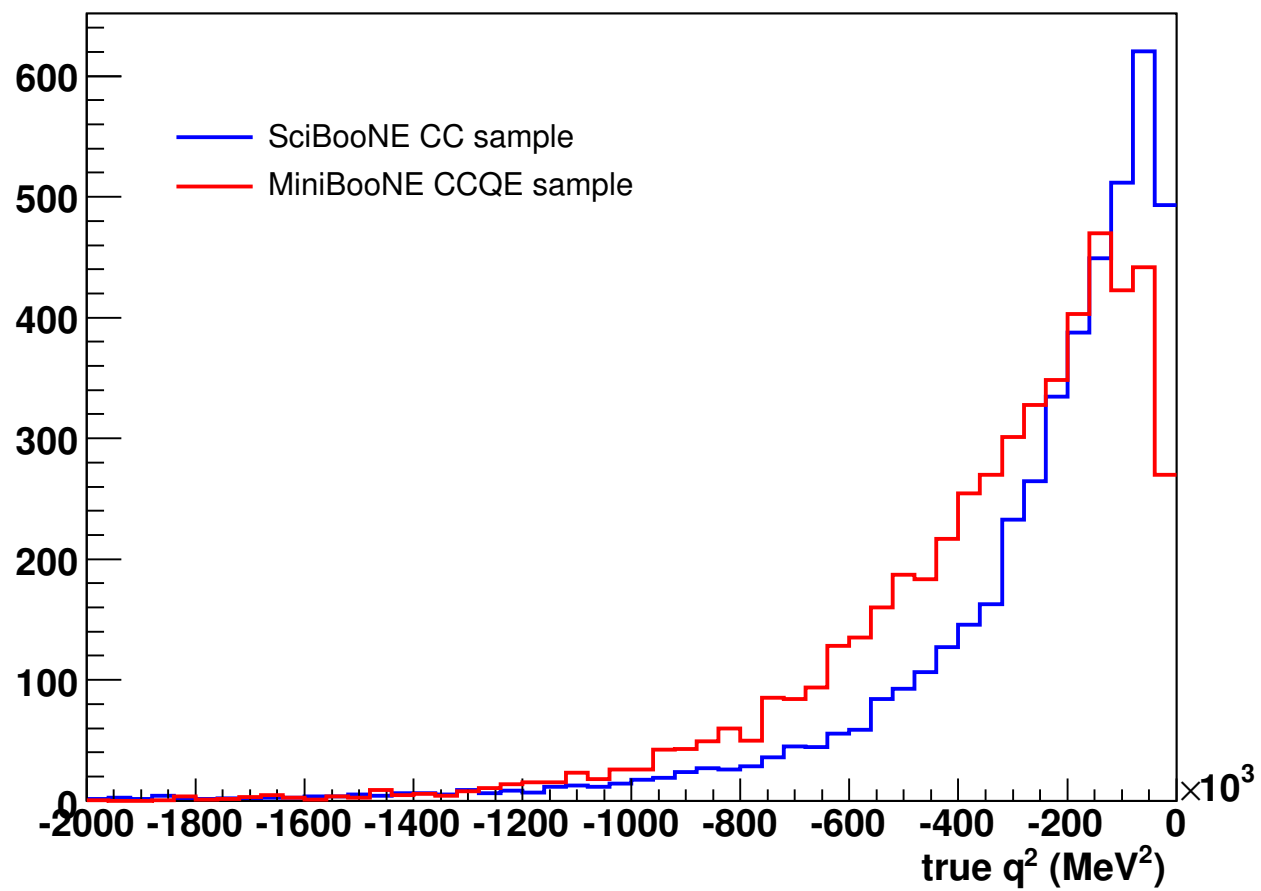

Figure 5.14: CCQE events (left) and CC $\pi^{+}$events (right) in the MiniBooNE CCQE sample (red) with the SciBooNE CC sample (blue). The two samples are normalized to equal POT. 


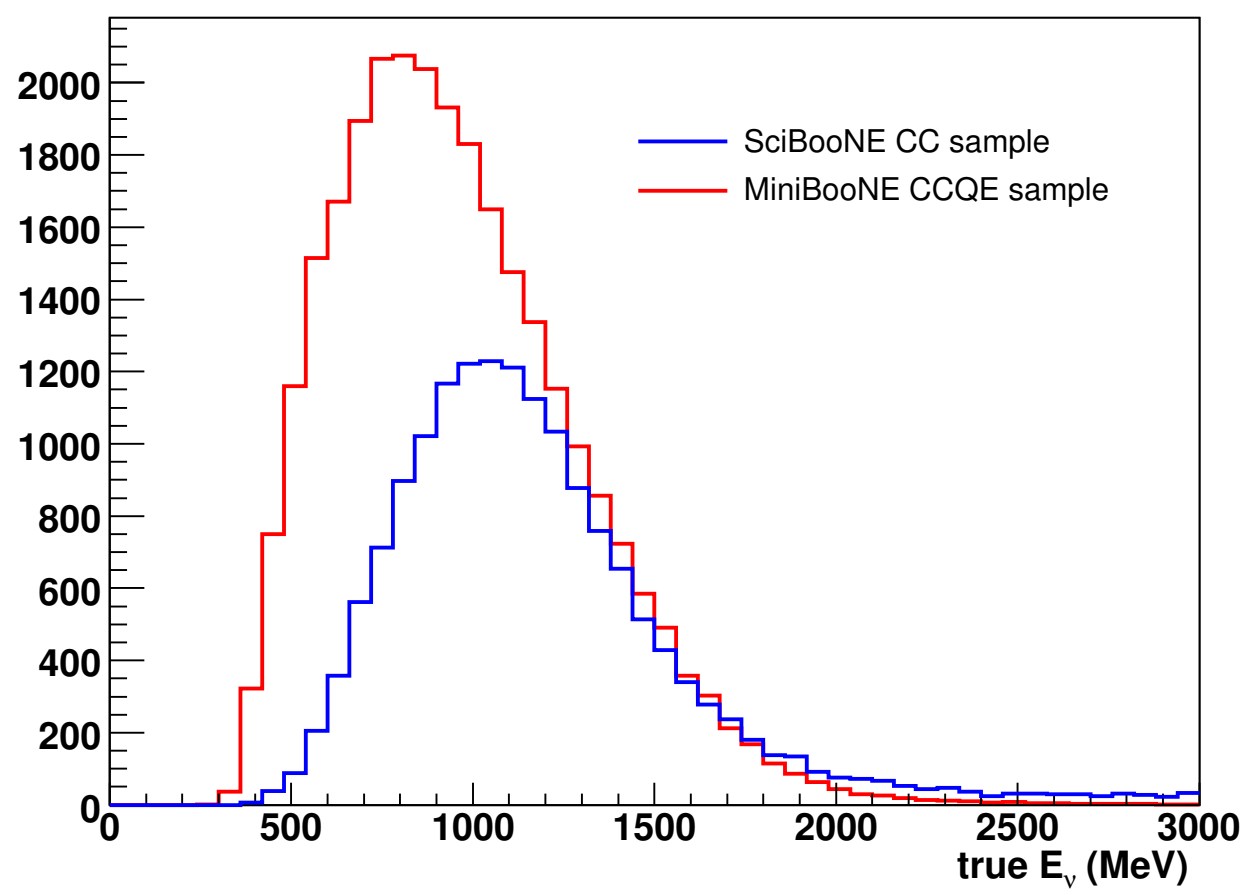

Figure 5.15: Generated neutrino energy $(\mathrm{MeV})$ in the MiniBooNE CCQE sample (red) with the SciBooNE CC sample (blue). The two samples are normalized to equal POT. 


\section{Chapter 6}

\section{$\nu_{\mu}$ Disappearance with MiniBooNE}

\subsection{Disappearance Search Method}

For a detector, such as MiniBooNE, located a fixed distance from a neutrino source, $\nu_{\mu}$ disappearance due to oscillations has a distinct signature in neutrino energy, because neutrinos with different energies oscillate with different probabilities for the same distance traveled.

The probability for disappearance (Section 2.2.1, Eq. 2.4) assumes two-flavor mixing $\left(\nu_{\mu} \rightarrow \nu_{x}\right)$ characterized with a single mass splitting $\Delta m^{2}$ between a heavy mass state and the three light neutrino mass states. Each neutrino is weighted with this probability based on the distance, $\mathrm{L}$, and energy, $\mathrm{E}$, of the neutrino. $\mathrm{L}$ is calculated from the decay point of the parent particles and the interaction point in the tank.

Figure 6.1 shows the ratio of the unoscillated MiniBooNE neutrino energy spectrum to the oscillated spectrum for various choices of $\Delta m^{2}$. Disappearance reduces the total number of events, proportional to $\sin ^{2}(2 \theta)$. In addition, different energies disappear differently, and so distinct shapes emerge as a function of neutrino energy depending on the value of $\Delta m^{2}$. Roughly, low $\Delta m^{2}<1 \mathrm{eV}^{2}$ creates disappearance at low $E_{\nu}$, and high $\Delta m^{2}>10 \mathrm{eV}^{2}$ create oscillations at high $E_{\nu}>1 \mathrm{GeV}$.

For CCQE events, the reconstructed energy of CCQE events is close to the true en- 
ergy, so oscillations over reconstructed energy bins (black dash) are similar to the true neutrino energy (red), with some smearing of the oscillation modes as events are placed in the wrong energy bin. The effect of adding in background $\mathrm{CC} \pi^{+}$events (blue) further reduces the magnitude of the oscillation. $\mathrm{CC} \pi^{+}$events also come from $\nu_{\mu}$ interactions, and therefore oscillate, but such events reconstruct at a lower energy than the generated energy (Fig. 5.2.6). As a result, the background $\mathrm{CC} \pi^{+}$are predominantly at low $E_{\nu}^{Q E}$, which reduces the ability to resolve disappearance at $\Delta m^{2} \sim 1 \mathrm{eV}^{2}$.

Disappearance would be observable either from a deficit of events (normalization) or, alternatively, from a distortion of the neutrino energy spectrum (shape) or both (shape + normalization). With the choice of CCQE cross section parameter $M_{A}=1.015 \mathrm{GeV}$, the ratio of detected events to predicted events in MiniBooNE for neutrinos is $1.31 \pm 0.26$ $(1.18 \pm 0.18$ for antineutrinos) which shows agreement within the uncertainties discussed later in this section. The absolute normalization uncertainties in a single detector experiment such as MiniBooNE are large, hence a shape-only disappearance fit is performed for the MiniBooNE only analysis. The joint MiniBooNE/SciBooNE analysis includes both normalization and shape information.

To test for disappearance, one compares the observed neutrino energy spectrum in MiniBooNE data to various oscillation predictions to evaluate how consistent the data is with a given oscillation prediction. Data and prediction are binned into $16 E_{\nu}^{Q E}$ bins, with the bin delimiters given in Table 6.1. The binning was chosen based on the $100 \mathrm{MeV}$ energy resolution in MiniBooNE.

One forms a $\chi^{2}$ between data in $E_{\nu}^{Q E}$ bin $i\left(d_{i}\right)$ to a prediction in the same energy bin $p_{i}$. The prediction includes underlying $\left(\Delta m^{2}, \sin ^{2}(2 \theta)\right)$ oscillation, where each $\nu_{\mu}$ event is weighted assuming an oscillation probability given in Eq. 2.4 for the events true neutrino energy and distance traveled.

$$
\chi^{2}=\sum_{i, j}^{16 b i n s}\left(d_{i}-N p_{i}\right) M_{i j}^{-1}\left(d_{j}-N p_{j}\right)
$$

where $N=\frac{\sum_{i} d_{i}}{\sum_{i} p_{i}}$ renormalizes the prediction to the total number of data events in the case of a shape-only fit and $N=1$ for a shape+normalization fit. The error matrix, $M_{i j}$, can either include shape and normalization information, or $M$ can be the shape only part 

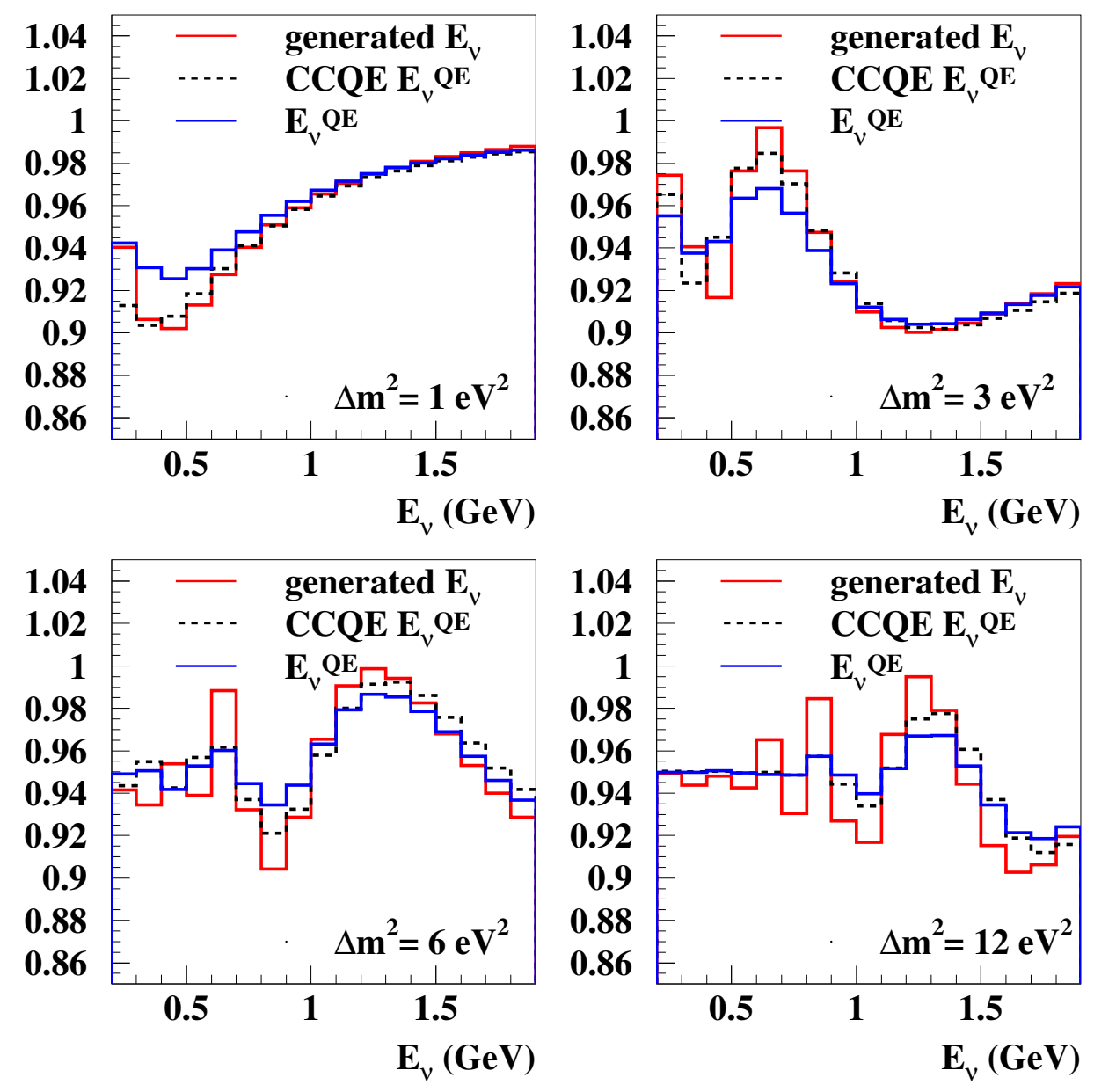

Figure 6.1: Ratio of disappearance scenarios to the unoscillated spectrum as a function of neutrino energy. Oscillations vs. generated neutrino energy (red), reconstructed neutrino energy for CCQE events (black dashed) and reconstructed neutrino energy for CCQE and $\mathrm{CC} \pi^{+}$events (blue) are shown. Top left: Disappearance corresponding to $\sin ^{2}(2 \theta)=0.1$ and $\Delta m^{2}=1.0 \mathrm{eV}^{2}$. Top right: Disappearance corresponding to $\sin ^{2}(2 \theta)=0.1$ and $\Delta m^{2}=3.0 \mathrm{eV}^{2}$. Bottom left: Disappearance corresponding to $\sin ^{2}(2 \theta)=0.1$ and $\Delta m^{2}=6.0 \mathrm{eV}^{2}$. Bottom right: Disappearance corresponding to $\sin ^{2}(2 \theta)=0.1$ and $\Delta m^{2}=12.0 \mathrm{eV}^{2}$. 


\begin{tabular}{|c|c|c|c|c|c|c|c|c|c|c|c|c|c|c|c|c|}
\hline 1 & 2 & 3 & 4 & 5 & 6 & 7 & 8 & 9 & 10 & 11 & 12 & 13 & 14 & 15 & 16 & 17 \\
\hline 0. & 0.4 & 0.5 & 0.6 & 0.7 & 0.8 & 0.9 & 1.0 & 1.1 & 1.2 & 1.3 & 1.4 & 1.5 & 1.6 & 1.7 & 1.8 & 1.9 \\
\hline
\end{tabular}

Table 6.1: Bin delimiters for the fit code, used for both $\nu_{\mu}$ and $\bar{\nu}_{\mu}$ analyses, in GeV.

of the error matrix for a shape fit.

First, the formulation of the error matrix is discussed in Section 6.2, and how it can be separated into shape and normalization components. Second, how the $\chi^{2}$ is used to perform fits is discussed in Section 6.4.

\subsection{Producing Error Matrices}

The error matrix encodes the error in each energy bin, as well as correlations between energy bins. Each entry is the size of the error in terms of number of events. A fractional error matrix is a percentage change squared in each bin, and is multiplied up by the number of events in each bin to form the "full" error matrix. For the analyses here, the predicted number of events in reconstructed neutrino energy bins, $p_{i}$, is used to form the systematic error matrix: $M^{\text {full }}=M_{i, j}^{f r a c} p_{i} p_{j}$.

Some examples of error matrices:

An uncorrelated fractional error (10\%) error in two bins:

$$
M_{i, j}^{f r a c}=\left[\begin{array}{cc}
0.01 & 0 \\
0 & 0.01
\end{array}\right]
$$

A fully correlated normalization error of $10 \%$ :

$$
M_{i, j}^{f r a c}=\left[\begin{array}{cc}
0.01 & 0.01 \\
0.01 & 0.01
\end{array}\right]
$$

Eq. 6.3 is an example of a normalization error. If bin 1 increases by 10\%, the error matrix relating bin 1 to bin 2 says that bin 2 is expected to increase by the same amount, $10 \%$.

The power of correlations shows up in the $\chi^{2}$. Using a statistics only error matrix with 
A events in bin 1 , and $B$ in bin 2:

$$
M^{\text {stat }}=\left[\begin{array}{cc}
A & 0 \\
0 & B
\end{array}\right]
$$

and adding in the normalization part:

$$
M_{i, j}^{\text {norm }+ \text { stat }}=\left[\begin{array}{cc}
A+N & N \\
N & B+N
\end{array}\right]
$$

Inversion yields:

$$
M^{-1}=\frac{1}{(A+N)(B+N)-N^{2}}\left[\begin{array}{cc}
B+N & -N \\
-N & A+N
\end{array}\right]
$$

(Note: one cannot invert a fully correlated matrix such as Eq. 6.3, but one can invert the combination of a fully correlated matrix with statistical errors, such as Eq. 6.5.)

Assuming $\Delta_{i}=d_{i}-p_{i}$, the $\chi^{2}$ is then:

$$
\chi^{2}=f\left[\Delta_{1}(B+N)-2 N \Delta_{1} \Delta_{2}+\Delta_{2}(A+N)\right]=f\left[\Delta_{1}^{2}(B+A)\right]
$$

where $f=1 /(A B+N B+A N)$. A normalization error "expects" that a variation in bin a between data and prediction $\left(\Delta_{1}\right)$ should be the same as a variation in bin $\mathrm{b}\left(\Delta_{1}\right)$. If $\Delta_{1}=\Delta_{2}$, there is no contribution to the $\chi^{2}$ due to the normalization error, N. If the $\chi^{2}$ was calculated with just statistical errors, the result would be: $\chi^{2}=\frac{1}{A B}\left[\Delta_{1}^{2}(B+A)\right]$; the only difference is the factor in front is smaller for the normalization case. This is because there is extra information, i.e. that the two bins should be similar. Correlations behave like pull terms in the $\chi^{2}$, and reduce the $\chi^{2}$ for normalization shifts. However, any residual difference which is not similar between bin 1 and bin 2 will increase the $\chi^{2}$.

A "shape" error matrix also has power to reduce the $\chi^{2}$. Here, the term "shape" means an error which is conserved across energy bins. A fluctuation allowed upwards in one bin must be compensated for in other bins. This can be like a resolution or energy scale error, where events migrate into neighboring bins but the total number of events is conserved. This is an anti-correlated error matrix.

A simple shape error matrix moves $10 \%$ of events from one bin into another bin:

$$
M_{\text {shape }}=\left[\begin{array}{cc}
0.01 & -0.01 \\
-0.01 & 0.01
\end{array}\right]
$$


This matrix behaves like a normalization error in the $\chi^{2}$. The $\chi^{2}$ will be small if the number of extra events in bin 1 is compensated by a lack of events of the same size in bin 2 .

\subsubsection{Shape and Normalization Error Matrices}

One can separate the shape and normalization components from any given error matrix $M_{i, j}$. The normalization part, $M_{i, j}^{n o r m}$, is the component of the error matrix which is the same for each element, and is is equivalent to the error on a one bin sample:

$$
M_{i, j}^{n o r m}=p_{i} p_{j} \frac{\sum_{l=1}^{n b i n} \sum_{k=1}^{n b i n} M_{k l}}{p_{t}^{2}}
$$

where $p_{t}=\sum_{i}^{n b i n} p_{i}$, is the prediction summed over all bins. The total number of bins is denoted nbin.

The shape part of the error matrix, $M_{i, j}^{\text {shape }}$, are allowed fluctuations where the total number of events is kept fixed across all bins.

$$
M_{i, j}^{\text {shape }}=M_{i j}-\frac{p_{j}}{p_{t}} \sum_{k=1}^{n b i n} M_{i k}-\frac{p_{i}}{p_{t}} \sum_{k=1}^{n b i n} M_{k j}+\frac{p_{i} p_{j}}{p_{t}^{2}} \sum_{k, l=1}^{n b i n} M_{k l}
$$

Appendix B provides checks of $M^{\text {shape }}$ as a representation of shape error.

\subsubsection{Creating An Error Matrix}

Assume there is a single source of uncertainty in parameter $\alpha \pm \delta_{\alpha}$ (e.g. $\alpha$ represents a measurement of attenuation length or a change to the POT normalization). To understand the effect $\alpha$ has on the energy spectrum, create two predictions, one with the default value of $\alpha, p_{i}^{\alpha}$, and one with $\alpha^{\prime}=\alpha+\delta_{\alpha}$, denoted $p_{i}^{\delta}$. The $p_{i}^{\alpha}\left(p_{i}^{\delta}\right)$ values are the correlated set of predicted number of events in each of the energy bins.

The error matrix which represents the correlated error between energy bins $i$ and $j$ due to $\alpha \pm \delta_{\alpha}$ is:

$$
M_{i j}^{\alpha}=\left(p_{i}^{\alpha}-p_{i}^{\delta}\right)\left(p_{j}^{\alpha}-p_{j}^{\delta}\right)
$$

If $\alpha$ is a POT normalization error, then $p_{i}^{\alpha}-p_{i}^{\delta}=n$ is the same for every bin $i$, so $M_{i j}^{\alpha}=$ $n$ for all $i, j$, and is a normalization error. If $\alpha$ has no effect on the energy distribution, then 
$p_{i}^{\alpha}-p_{i}^{\delta}=0$ and the error matrix is 0 . In MiniBooNE jargon, this method of forming an error matrix is called a "unisim", where one parameter is varied at a time in accordance with its uncertainty.

In principle, one could vary each source of systematic uncertainty one by one and rerun the entire prediction with only one change. In practice, it is difficult to change each systematic independent of all other systematics, as systematic uncertainties can be correlated. An example: If the total amount of light is constrained by calibration, then the scintillation light and Cherenkov light cannot both be increased simultaneously. Thus, the scintillation fraction and Cherenkov fraction must be changed together.

To account for correlations, one employs a second method of forming error matrices, called "multisims". Instead of varying one parameter $\alpha$, one varies a set of $\vec{\alpha}=$ $\left(\alpha_{1}, \alpha_{2}, \ldots, \alpha_{N}\right)$ in a correlated way. In the example, instead of varying scintillation and Cherenkov light individually, one would instead draw from an allowed region which relates scintillation light to Cherenkov light with their individual errors while requiring the total amount of light to be fixed. Such a region is like the shape matrix discussed earlier, where an increase in the scintillation light comes at an equal decrease to the Cherenkov light.

Create a prediction $p^{k}$, for each draw $\vec{\alpha}_{k}$ from the allowed parameter region. In the example, one would draw the scintillation fraction $f_{s}$ and the Cherenkov fraction $f_{c}$ together and ensure the total light was constant. Three such draws might look like: $f_{s}=(0.5,0.7,0.2), f_{c}=(0.5,0.3,0.8)$.

The error matrix which describes the effect of variations to $\vec{\alpha}$ within the allowed region:

$$
M_{i j}^{\vec{\alpha}}=\sum_{k}^{N}\left(p_{i}^{c v}-p_{i}^{k}\right)\left(p_{j}^{c v}-p_{j}^{k}\right)
$$

where a total of $N$ draws were used, and $p^{c v}$ represents a "central value" prediction using the default set of $\vec{\alpha}$. For a Gaussian distributed error of $\alpha \pm \delta_{\alpha}$ considered in the unisim case, one draw is sufficient; the advantage to the multisim method is if the source of uncertainty is nonlinear or $\vec{\alpha}$ are correlated. In the case where there is 1 parameter and 1 energy bin, 1000 draws would map out the effect on the energy due to the parameter 
to the $\sim 1 \%$ level. For the analysis used here, 1000 draws were used to form most error matrices. Appendix $C$ discusses the number of draws needed to build an error matrix; 100 draws provides a reasonable estimate of the true error matrix but 1000 draws is preferred when possible.

To run multisims (hundreds or thousands of draws of $\vec{\alpha}$ ), would require substantial computing resources if the flux, cross section and detector simulation were rerun for each draw. To avoid this problem, one uses "reweighting" to calculate a weight for the event, instead of a new event. For all events, important underlying parameter information is stored (e.g. neutrino energy, distance traveled, cross section interaction type and kinematics, neutrino parent type and kinematics, muon kinematics). Now, instead of rerunning the entire simulation, a weight, $w t_{k}$, is applied to each neutrino event based on the change due to a particular systematic uncertainty. The weight is then applied for all events to form $p_{i}^{k}$. The weight is the effect of changing underlying parameters (e.g. the ratio of the cross section with a change to the axial mass, $\left.w t=\frac{\sigma\left(M_{A}=1.23\right)}{\sigma\left(M_{A}=1.00\right)}\right)$. Reweighting is used for the flux and cross section systematics, and some detector systematics.

\subsection{Disappearance Fit Error Matrix}

The total error matrix, $M$, is the sum of the individual systematic error matrices and the statistical error matrix, $M=M^{\text {flux }}+M^{\text {cross section }}+M^{\text {detector }}+M^{\text {statistics }}$. First, the formation of the flux, cross section and detector error matrices are described in Sections 6.3.1-6.3.3. Section 6.3.4 summarizes the systematic error matrix components used for the $\nu_{\mu}$ disappearance fit.

\subsubsection{Flux Uncertainties}

The sources of uncertainty considered in the flux error matrix are:

1. absolute POT normalization (Section 3.2)

2. $\mathrm{p}$-Be hadronic cross sections (Section 4.1.3)

3. Production of pions off the Be target (Section 4.1.4) 
4. Production of kaons off the Be target (Section 4.1.4)

5. Magnetic field produced by the horn (Section 4.1.5)

The dominant uncertainties are (3) pion production off the target and (5) the magnetic field which focuses the pions.

Parent pion uncertainties are calculated using the HARP experiment data and covariance matrix. First, a draw from the HARP covariance matrix provides an alternate HARP universe consistent with HARP. A spline is created from the new HARP dataset to interpolate across relevant $p_{\pi^{+}}$and $\theta_{\pi^{+}}$values. Each neutrino event is weighted the ratio of $w t=\frac{\sigma_{\text {spline }}}{\sigma_{S W}}$, where the new $\pi^{+}$cross section is calculated from the spline, and $\sigma_{S W}$ is the cross section according to the default Sanford-Wang parameterization (Section 4.1.4). The prediction, $p_{i}^{k}$, is formed by applying the weights to all neutrino events and plotting them as a function of reconstructed neutrino energy. For this procedure, 1000 draws are used for 70k events; running over more events does not appreciably change the resulting matrix. These draws are compared to the default prediction which employs the SW parameterization, $p_{i}^{c v}$, and the error matrix formed as detailed in Eq. 6.12. Similarly, to produce an error matrix due to $\pi^{-}$production, the $\pi^{-}$HARP dataset and covariance matrix are used.

The parent kaon uncertainties are formed by taking draws from the covariance matrix shown in Table 4.3. The weight for the neutrino events is $w t=\frac{\sigma_{c_{i}^{k}}}{\sigma_{F S}}$ the ratio of the new cross section with the drawn $c_{i}^{k}$ to the default $c_{i}^{F S}$ values (Table 4.3). The weights are applied to the neutrino spectrum to produce a new histogram prediction $p_{i}^{k}$, and the error matrix calculated using Eq. 6.12. This error matrix is negligible in comparison to the $\pi^{-}$ production error matrix.

The horn magnetic field and hadronic cross section errors are produced by rerunning the beam simulation with appropriate changes. A series of histograms are produced as a function of the generated neutrino energy and neutrino parent type for each simulation. This avoids the problem of running the cross section and detector simulations, as neutrino production uncertainties only depend on the neutrino energy and parent type. The weight applied to form is $p_{i}^{k}$ is the ratio of the histograms, and the uncertainty is calculated using the unisim method (Eq. 6.11). 
The POT uncertainty is a $2 \%$ normalization error matrix, $M_{i j}^{P O T}=(0.02)^{2} p_{i}^{c v} p_{j}^{c v}$

\subsubsection{Cross Section Uncertainties}

The sources of uncertainty considered in the cross sections:

- CCQE cross section: $M_{A}=1.015 \pm 0.23 \mathrm{GeV}, \kappa=1.000 \pm 0.019, E_{B}=34 \pm 9 \mathrm{MeV}$ and $p_{F}=220 \pm 30 \mathrm{MeV} / \mathrm{c}$ (Section 4.2.1)

- CCQE cross section model uncertainty: 10\% (Section 4.2.1)

- $\mathrm{CC} \pi$ cross section: $M_{A}^{1 \pi}=1.10 \pm 0.275 \mathrm{GeV}$ (Section 4.2.2)

- FSI effects: $\pi$ absorption, $\pi$ charge exchange, pionless $\Delta$ decay in the nucleus (Section 4.2.3)

The NUANCE cross section parametrization can be used in the MiniBooNE analysis framework, so for any cross section variation, a new cross section with a parameter change can be calculated. $M_{A}, \kappa, E_{B}, p_{F}, M_{A}^{1 \pi}$ are all fluctuated to form a weight of the ratio of the new cross section to the old. For a variation to $M_{A}$, the weight would be $w t=\frac{\sigma\left(M_{A}=1.23\right)}{\sigma\left(M_{A}=1.00\right)}$. The error matrix is then formed using Eq. 6.12.

The only uncertainty this method does not work for are the FSI interactions in the nucleus. For example, the absorption of a pion can cause a $\mathrm{CC} \pi^{+}$event to pass CCQE selection cuts. To properly account for the migration of these events, the nuclear effect changes were randomly drawn in the detector simulation uncertainty draws (discussed in Section 6.3.3). As a result, the detector uncertainties can be thought of as inclusive detector and cross section final state uncertainties together.

\subsubsection{Detector Uncertainties}

There are three sources of detector uncertainties:

- Optical model: light propagation, scattering, reflection and PMT effects (Section 3.4)

- Electronics model (charge/time slewing) (Section 3.4) 
- Nuclear effects in the detector: $\pi$ absorption, $\pi$ charge exchange in the oil (Section 4.3.2)

The optical model of MiniBooNE uses 39 parameters, which describe the various optical properties in the detector, including attenuation length, scattering (Rayleigh and Raman), PMT efficiencies, reflections, and multiple scintillation and fluorescence components of the oil.

The correlations between individual optical parameters is described by a covariance matrix, developed iteratively. An initial covariance matrix for the optical model, $O$, with mean $\bar{o}$, was based off initial measurements of each parameter $\left(o_{i}\right)$ (discussed in Section 3.4). Then, draws from $O$ produce a set of $o_{i}^{k}$, where $k=1,3000$. For each draw, a set of electrons from muon decay are generated in the detector simulation. This can be thought of as 3000 universes with slightly different detectors, each consistent with the initial estimate of mineral oil, PMT and detector properties. A high statistics sample of electrons from muon decay is collected from cosmic ray events, and a $\chi^{2}$ is formed between the data and the prediction, $p_{i}^{k}$, for energy and other reconstructed quantities. A weight, $\eta^{k}$, for each parameter set draw, $\vec{o}^{k}$, is calculated based on the $\chi^{2}$ of the prediction compared to data (statistical errors only) and the likelihood to draw the set $\vec{o}^{k}$ from $O$. A new covariance matrix, $O^{\prime}$ and mean, $\bar{o}^{\prime}$, is formed by applying the weight:

$$
O^{\prime}{ }_{i j}=\frac{\sum_{k}^{3000} \eta^{k}\left(o_{i}^{k}-\bar{o}_{i}^{\prime}\right)\left(o_{j}^{k}-\bar{o}_{j}{ }_{j}\right)}{\sum_{k}^{3000} \eta^{k}}
$$

The same procedure is applied to $O^{\prime}$ as was applied to $O$, until the agreement between data and the draws ceases to improve. The final covariance matrix for the optical parameters is produced after approximately 20 iterations. More details can be found under Ref. [108].

From the final optical parameter covariance matrix, 80 parameter sets are drawn and the full detector simulation run. In addition, the cross section FSI uncertainties are also drawn. Each simulation corresponds to approximately data-size statistics. The error matrix for the detector uncertainties (and FSI) is then produced using Eq. 6.12, by comparing the default prediction to the predictions assuming different optical model parameters. 
The error matrix due to electronics and DAQ model variations is calculated using the unisim method. Predictions are run with changes to the underlying electronics model and DAQ and high $(\sim 7 \times)$ data statistics and compared to the default to form the error matrix.

The uncertainty on nuclear effects in the detector is calculated using reweighting. Events are tagged in the detector simulation as having undergone $\pi$ absorption or charge exchange. Only the tagged events are weighted to form a prediction $(+35 \%$ for absorption, $+50 \%$ for charge exchange) which is compared to the default to form the error matrix.

\subsubsection{Summary of Uncertainties}

Three qualities will be used to understand the flux, cross section and detector error matrices:

- The normalization of the error matrix (Eq. 6.9)

- The diagonal elements of the shape-only error matrix (Eq. 6.10)

- The correlations of the shape-only error matrix $\left(\rho_{i j}=\frac{M_{i j}^{\text {shape }}}{\sqrt{M_{i i} M_{j j}}}\right)$

A summary of the normalization error for each source of uncertainty is in Table 6.2. The dominant normalization uncertainty is the cross section uncertainty, specifically uncertainties on the CCQE cross section.

The (fractional) size of the diagonal elements of the shape-only error matrix (total, flux, cross section and detector) is shown in Fig. 6.2. While the diagonal elements are large, the correlations between energy bins is also large, as shown in Fig. 6.3. While these describe the shape-only error matrix, the measure of each error is its effect on the sensitivity to oscillation, which is discussed in the next section.

\subsection{Fit for $\nu_{\mu}$ Disappearance and Confidence Regions}

The search for neutrino oscillation in the disappearance channels uses a $\chi^{2}$ statistic to describe the agreement between data and prediction. Confidence level regions are determined by using frequentist methods to study the probability distribution for this $\chi^{2}$ statistic. 


\begin{tabular}{|c|c|c|}
\hline uncertainty type & source of uncertainty & normalization uncertainty \\
\hline \multirow[t]{6}{*}{ flux } & & $6.2 \%$ \\
\hline & $\pi^{+}$production & $4.0 \%$ \\
\hline & $K^{+}$production & $0.2 \%$ \\
\hline & horn magnetic field & $4.1 \%$ \\
\hline & p-Be hadronic cross sections & $1.0 \%$ \\
\hline & POT normalization & $2.0 \%$ \\
\hline \multirow[t]{5}{*}{ cross section } & & $19.1 \%$ \\
\hline & CCQE cross section $\left(M_{A}, \kappa, E_{B}, p_{F}\right)$ & $16.6 \%$ \\
\hline & CCQE cross section model dependence & $7.4 \%$ \\
\hline & CC $\pi$ cross section: $M_{A}^{1 \pi}$ & $5.6 \%$ \\
\hline & $\mathrm{CC} \pi$ cross section model dependence & $0.4 \%$ \\
\hline \multirow[t]{4}{*}{ detector } & & $3.4 \%$ \\
\hline & Optical model + FSI effects & $2.7 \%$ \\
\hline & Electronics model & $2.6 \%$ \\
\hline & Hadronic interactions in detector medium & $0.2 \%$ \\
\hline total & & $20.4 \%$ \\
\hline
\end{tabular}

Table 6.2: Summary of systematic uncertainties considered in the MiniBooNE disappearance fit. The third column indicates the normalization uncertainty due to each source of systematic error (Eq. 6.9). 


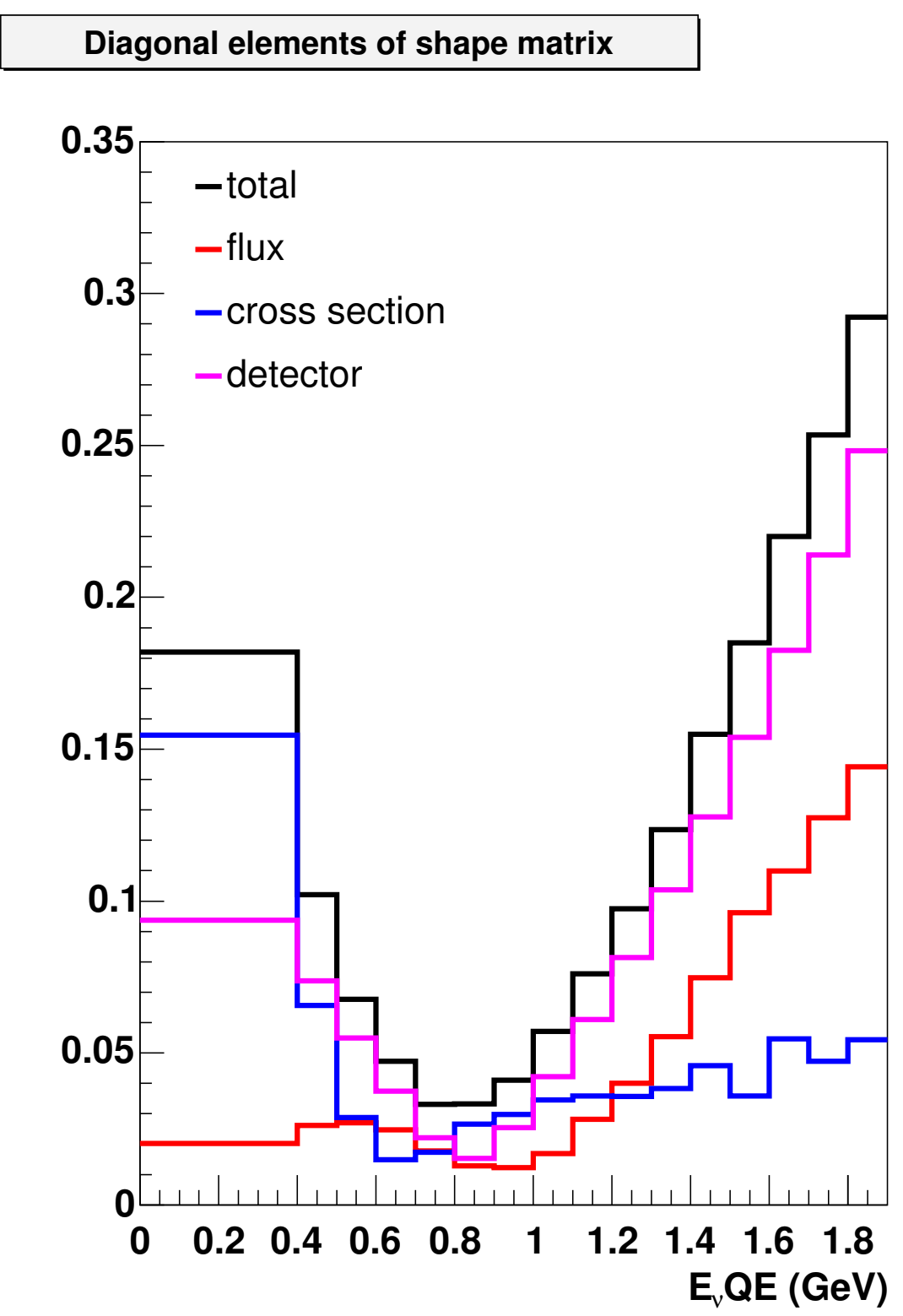

Figure 6.2: Diagonal elements of the shape-only error matrix $\left(\sqrt{M_{i i}^{\text {shape }}} / p_{i i}\right)$ vs. $E_{\nu}^{Q E}$. The shape-only error matrix is calculated using Eq. 6.10. The total shape-only error matrix (black) is the sum of the flux (red), cross section (blue) and detector (pink) error matrices. 


\section{Correlations of the shape-only error matrix}

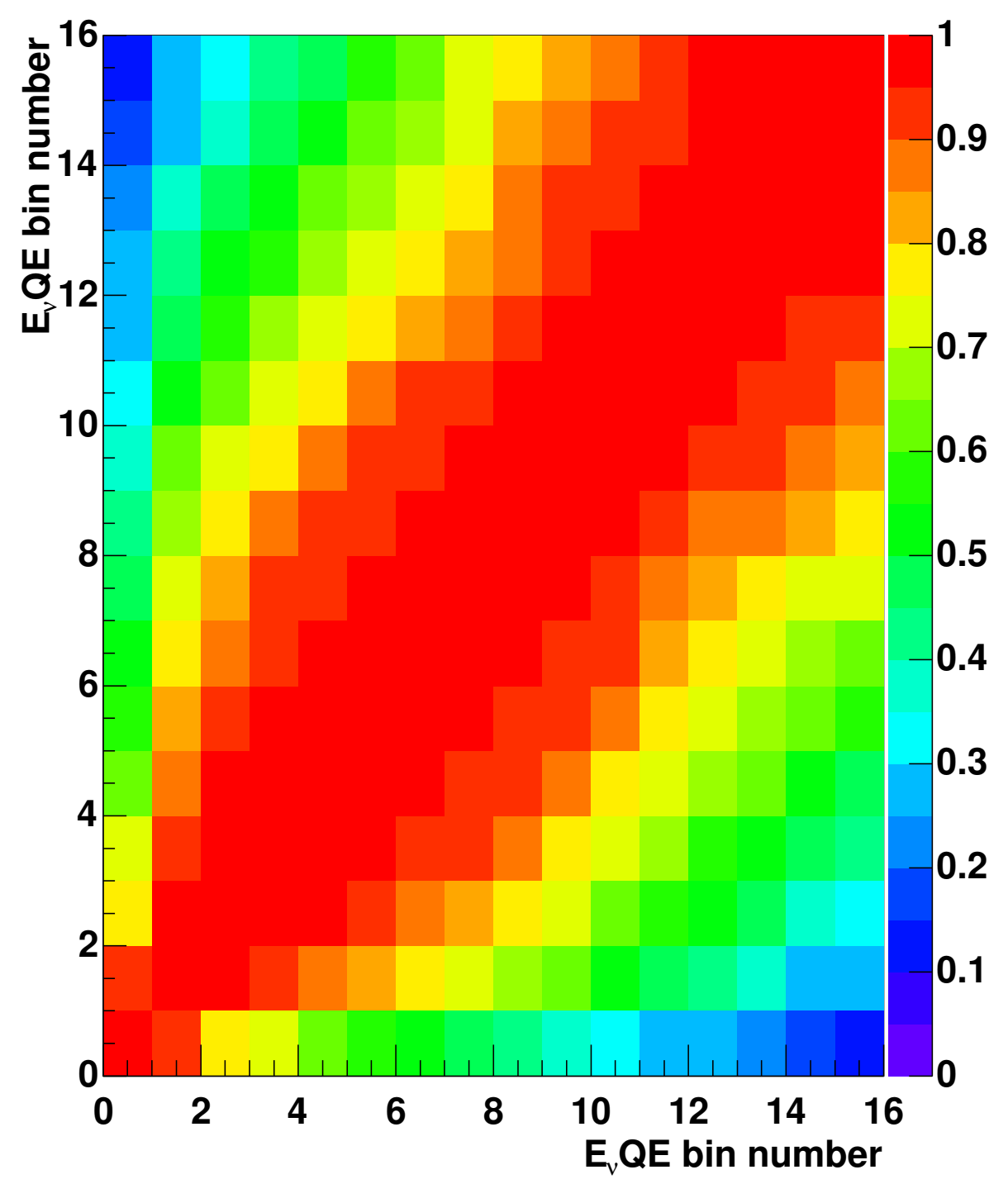

Figure 6.3: The correlation $\rho_{i j}=M_{i j}^{\text {shape }} / \sqrt{\left(M_{i i}^{\text {shape }} M_{j j}^{\text {shape }}\right)}$ of the total error matrix vs. $E_{\nu}^{Q E}$ bins. The shape-only error matrix is calculated using Eq. 6.10. 
The $\chi^{2}$ distribution for a series of "fake data" determines the regions of appropriate probability, or confidence level (CL) regions.

Fake data is generated with the following steps:

- Fluctuate the prediction using the total systematic error matrix

- Add statistical jitter in each bin based on the number of events there.

- Use the prediction to add an oscillation signal, if applicable.

For each fake data, the $\chi^{2}$ is calculated at $120 \times 120 \Delta m^{2}-\sin ^{2}(2 \theta)$ points between $0.1<\Delta m^{2}<100 \mathrm{eV}^{2}$ and $0.01<\sin ^{2}(2 \theta)<1.0$. The $\chi^{2}$ can either be calculated with the "true" matrix, i.e. the matrix generated with a prediction oscillated according to that point, or with the "best fit" matrix, the matrix at the point of minimum $\chi^{2}$ across the whole grid. The best fit point is found using the "true matrix".

Figure 6.4 shows the $\chi^{2}$ calculated at a point with negligible oscillation $\left(\Delta m^{2}=\right.$ $\left.0.2 \mathrm{eV}^{2}, \sin ^{2}(2 \theta)=0.014\right)$ for 50 fake data sets and the shape-only total error matrix. The $\chi^{2}$ fits well to a $\chi^{2}$ distribution of 16 degrees of freedom (DOF), corresponding to the 16 bins; the mean is consistent with 16 as well (15.2 \pm 0.8$)$. This implies that out of $50, \sim 5$ data sets should have $\chi^{2}>\chi^{2}(90 \% \mathrm{CL}, 16 \mathrm{DOF})=23.5$, which is the case.

Table 6.3 shows the fitted value of DOF to $\chi^{2}$ distributions formed with 50 fake data sets drawn and fit, according to a shape-only matrix generated at various $\Delta m^{2}-\sin ^{2}(2 \theta)$ points. If the draws are from a shape+normalization matrix but fit using a shape-only matrix, the results are similar. Across the $\Delta m^{2}-\sin ^{2}(2 \theta)$ surface, the $\chi^{2}$ DOF is constant and consistent with 16 DOF.

\subsubsection{Pearson's $\chi^{2}$ Method}

From these fake data studies, it is reasonable to use the $\chi^{2}$ as the statistics to form confidence regions. This procedure is called the Pearson's $\chi^{2}$ test. Specifically, to the procedure to create for allowed regions of disappearance with a fit to a dataset is given by: 


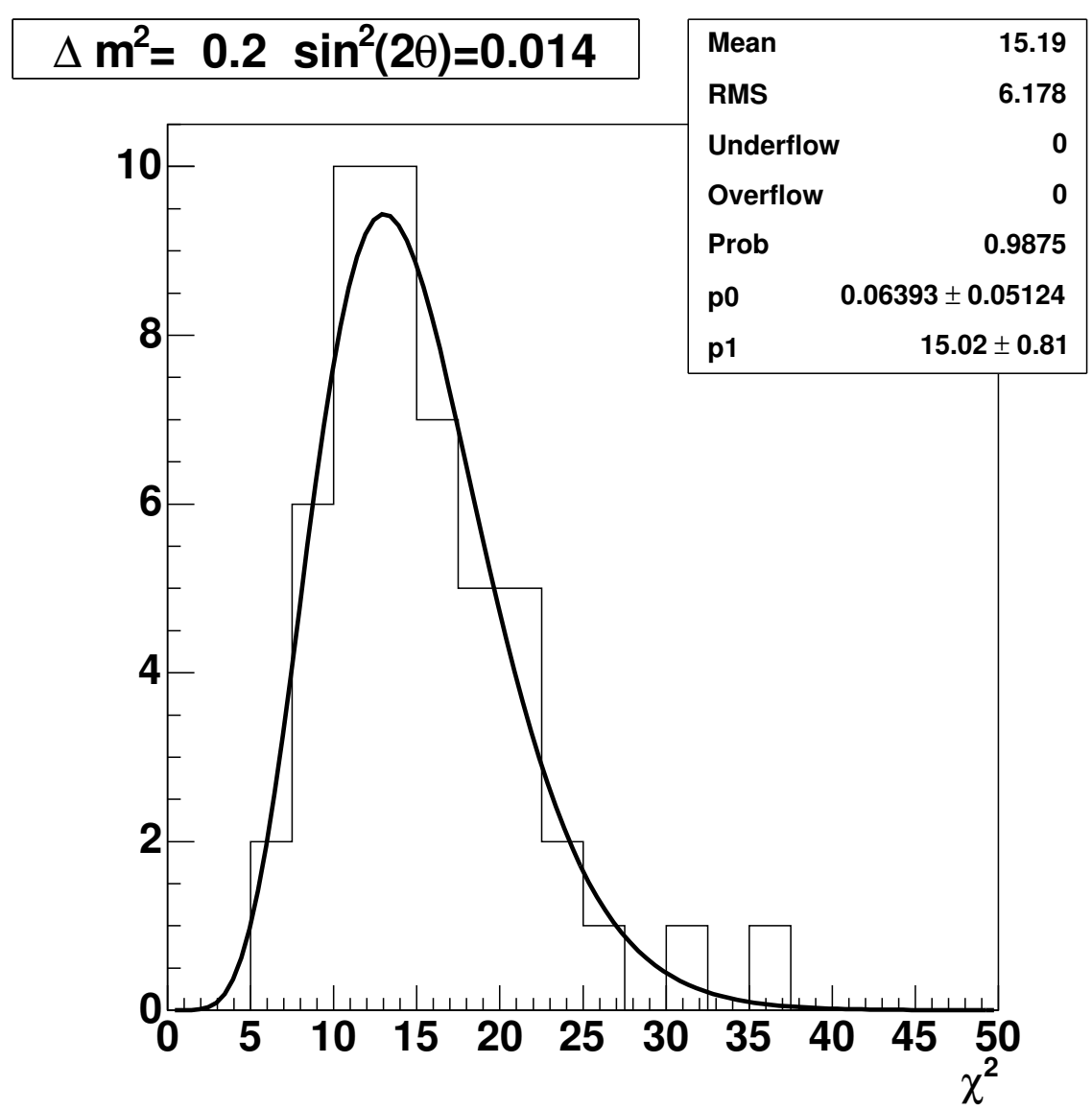

Figure 6.4: The $\chi^{2}$ distribution of 50 fake data experiments with negligible oscillation $\left(\Delta m^{2}=0.2 \mathrm{eV}^{2}, \sin ^{2}(2 \theta)=0.014\right)$. The shape-only error matrix is used to calculate the $\chi^{2}$. The black line shows a fit to a $\chi^{2}$ distribution with $15.02 \pm 0.81 \mathrm{DOF}$, and the mean is $15.2 \pm 0.8$. 


\begin{tabular}{|c||c|c|c|}
\hline$\chi^{2}$ DOF & $\sin ^{2}(2 \theta)=0.014$ & $\sin ^{2}(2 \theta)=0.098$ & $\sin ^{2}(2 \theta)=0.679$ \\
\hline \hline$\Delta m^{2}=56.0 \mathrm{eV}^{2}$ & $17.8 \pm 1.1$ & $17.7 \pm 1.3$ & $16.6 \pm 0.9$ \\
\hline$\Delta m^{2}=13.1 \mathrm{eV}^{2}$ & $15.0 \pm 1.2$ & $15.6 \pm 1.1$ & $16.8 \pm 1.1$ \\
\hline$\Delta m^{2}=3.1 \mathrm{eV}^{2}$ & $15.9 \pm 1.5$ & $16.2 \pm 1.2$ & $16.2 \pm 0.9$ \\
\hline$\Delta m^{2}=0.2 \mathrm{eV}^{2}$ & $15.2 \pm 0.8$ & $14.2 \pm 0.8$ & $16.5 \pm 0.9$ \\
\hline
\end{tabular}

Table 6.3: Fitted value of DOF to $\chi^{2}$ distributions formed with 50 fake data sets drawn and fit, according to a shape-only matrix generated at various $\Delta m^{2}-\sin ^{2}(2 \theta)$ points.

- Calculate the $\chi^{2}$ at each point in $\Delta m^{2}-\sin ^{2}(2 \theta)$ using prediction at that point to form the (shape-only) error matrix.

- For $\Delta m^{2}-\sin ^{2}(2 \theta)$ points where $\chi^{2}\left(\Delta m^{2}-\sin ^{2}(2 \theta)\right)>\chi^{2}(90 \% C L)$, that point is excluded at $90 \%(C L)$. The CL is determined acording to a $\chi^{2}$ statistic of $16 \mathrm{DOF}$.

For a $\chi^{2}$ distribution with $16 \mathrm{DOF}, 90 \%$ of the curve is contained within $\chi^{2}<23.5$, so $\chi^{2}\left(\Delta m^{2}-\sin ^{2}(2 \theta)\right)<23.5$ forms the $90 \%$ CL contour. The $3 \sigma$ contour corresponds to $\chi^{2}<36.2$, and $5 \sigma$ corresponds to $\chi^{2}<53.7$.

The sensitivity of an experiment can be estimated by a fit to "data" which exactly agrees with prediction and uses an error matrix that includes all statistical and systematic uncertainty. The $90 \%, 3 \sigma$ and $5 \sigma$ CL sensitivity contours for MiniBooNE are shown in Fig. 6.5 along with previous 90\% CL limits from the CDHS and CCFR experiments. Removing each source of error individually indicates which error has the most impact on the sensitivity most, and is shown in Fig. 6.6. The two largest errors are the flux $\left(\pi^{+}\right.$ prodiction) and cross section (CCQE cross section) uncertainties.

\subsection{2 $\Delta \chi^{2}$ Method}

A second statistic that can be used to evaluate CL regions is $\Delta \chi^{2}=\chi_{\text {true }}^{2}-\chi_{\text {bestfit }}^{2}$, where one compares the $\chi^{2}$ at the true point to the best fit across the entire $\Delta m^{2}-\sin ^{2}(2 \theta)$ surface. Figure 6.7 shows the $\chi^{2}$ distribution of best fit points for the 50 fake data sets with negligible oscillation ( $\Delta m^{2}=0.2 \mathrm{eV}^{2}, \sin ^{2}(2 \theta)=0.014$ ). The matrix used to calculate the $\chi_{\text {bestfit }}^{2}$ is the same as used to calculate the $\chi_{\text {true }}^{2}$ distribution shown in Fig. 6.4. Fig. 6.8 


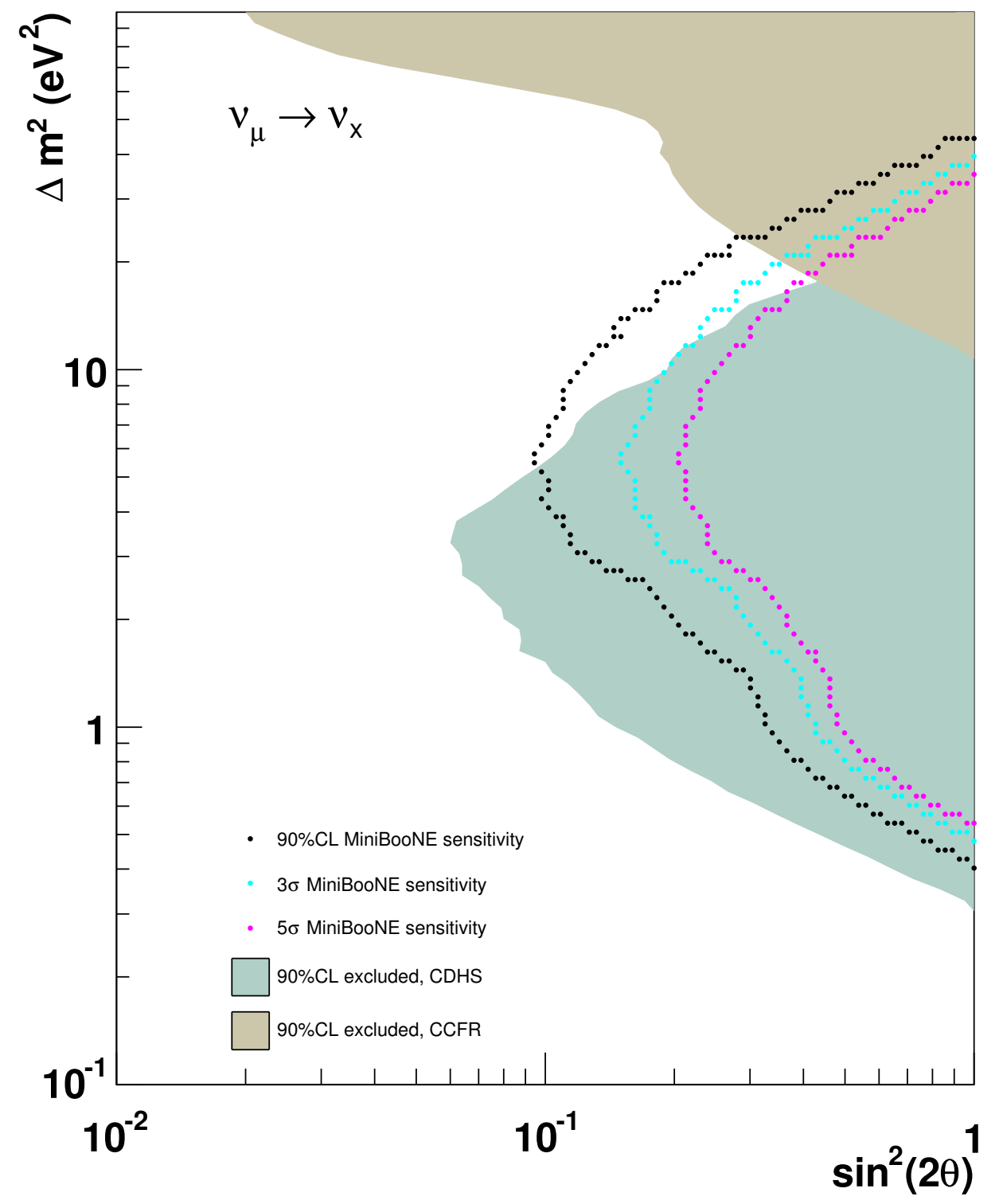

Figure 6.5: Pearson $\chi^{2}$ method 90\% CL sensitivity (black) $3 \sigma$ sensitivity (cyan) and $5 \sigma$ sensitivity (pink) for 5.579e20 POT. The 90\% CL exclusion regions for CCFR (light brown) and CDHS (light blue) are shown. 


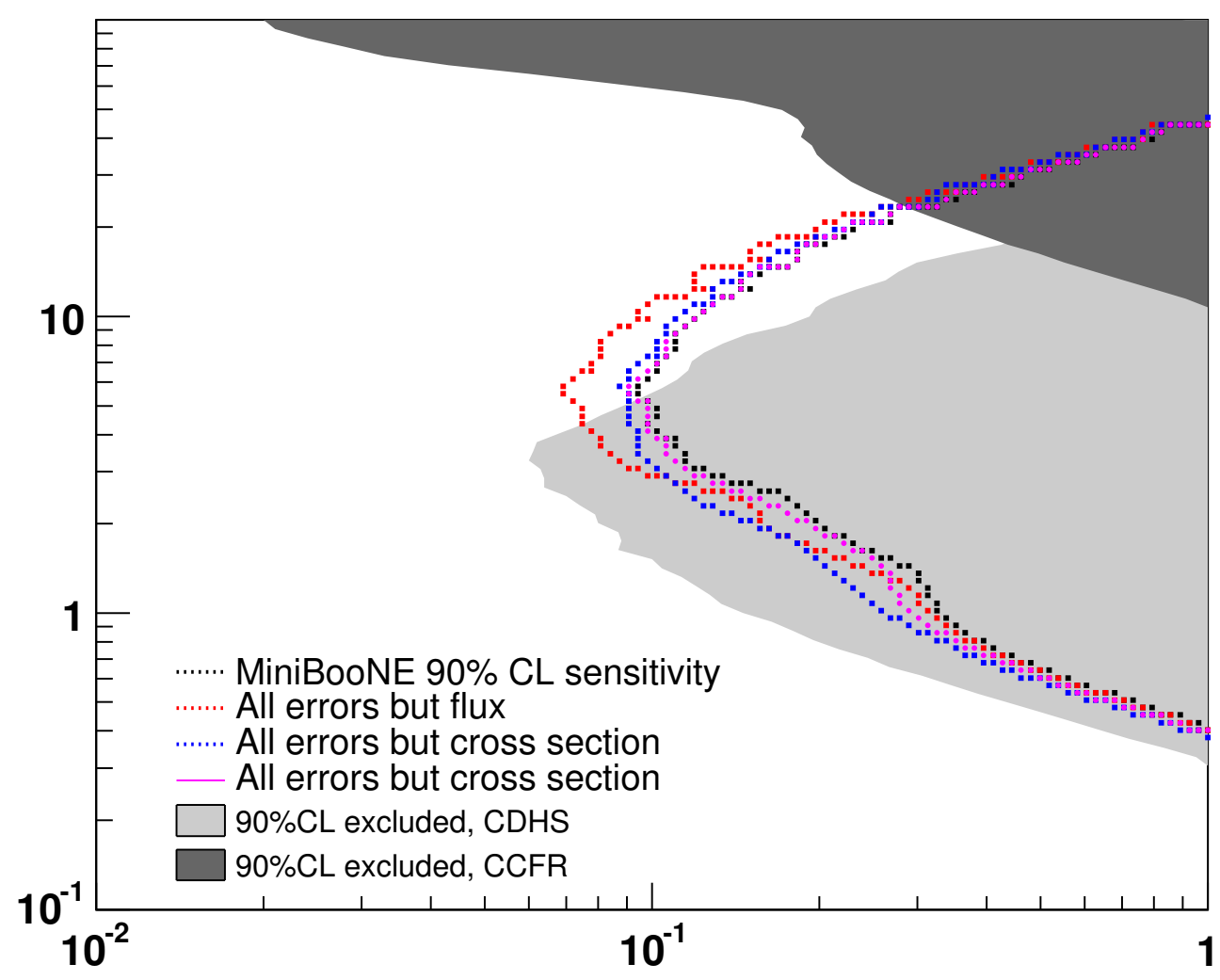

Figure 6.6: Pearson $\chi^{2}$ method 90\% CL sensitivities. All errors included is the $90 \%$ CL sensitivty (black). Each error contribution is removed from the total and replotted to demonstrate the effect of the error. All but flux uncertainties (red), cross sections (blue), and detector model (pink) are shown. The 90\% CL exclusion regions for CCFR (dark grey) and CDHS (light grey) are also shown. 


\begin{tabular}{|c||c|c|c|}
\hline$\Delta \chi^{2} \mathrm{DOF}$ & $\sin ^{2}(2 \theta)=0.014$ & $\sin ^{2}(2 \theta)=0.098$ & $\sin ^{2}(2 \theta)=0.679$ \\
\hline \hline$\Delta m^{2}=56.0 \mathrm{eV}^{2}$ & $5.4 \pm 0.8$ & $5.0 \pm 0.5$ & $4.2 \pm 0.4$ \\
\hline$\Delta m^{2}=13.1 \mathrm{eV}^{2}$ & $4.3 \pm 0.4$ & $4.3 \pm 0.7$ & 0 \\
\hline$\Delta m^{2}=3.1 \mathrm{eV}^{2}$ & $5.3 \pm 0.5$ & $2.8 \pm 0.8$ & 0 \\
\hline$\Delta m^{2}=0.2 \mathrm{eV}^{2}$ & $4.5 \pm 0.4$ & $4.4 \pm 0.8$ & $4.4 \pm 0.7$ \\
\hline
\end{tabular}

Table 6.4: Fitted value of DOF to $\Delta \chi^{2}$ distributions formed with 50 fake data sets drawn and fit, according to a shape-only matrix generated at various $\Delta m^{2}-\sin ^{2}(2 \theta)$ points.

shows the $\Delta \chi^{2}$ distribution for $\Delta m^{2}=0.2 \mathrm{eV}^{2}, \sin ^{2}(2 \theta)=0.014$.

For a fit to two parameters $\left(\Delta m^{2}\right.$ and $\left.\sin ^{2}(2 \theta)\right)$, the $\Delta \chi^{2}$ surface should be flat, and follow a $\chi^{2}$ distribution corresponding to 2 DOF (4.61 for $90 \%$ CL). However, the $\Delta \chi^{2}$ distribution appears to correspond to a $\chi^{2}$ of $\sim 4$ DOF. Table 6.4 gives the $\Delta \chi^{2}$ fitted DOF across $\Delta m^{2}-\sin ^{2}(2 \theta)$ surface; the $\Delta \chi^{2}$ value varies between 3-5 DOF. Within the sensitive region, the $\chi^{2}$ at the best fit finds the true point (the $\chi^{2}$ of nearby points is worse) so the $\Delta \chi^{2}=0$.

The reason that the $\Delta \chi^{2}$ does not correspond to expectation is because of the $\chi^{2}$ at the best fit. The location of the best fit point tends to deviate from the true point and cluster near the edges of the sensitive region, as shown in Fig. 6.9. Figure 6.10 shows a particular fake data set compared to the null prediction, with statistical errors shown. The shape-only $\chi^{2}$ between the fake data set and prediction with no oscillation is acceptable $\left(\chi^{2}=12.3\right)$. The oscillations are able to match statistical fluctuations in the fake data, and reduce the $\chi^{2}$; the $\chi^{2}$ at the best fit of $\Delta m^{2}=15.6 \mathrm{eV}^{2}, \sin ^{2}(2 \theta)=0.124$ is 8.6. As a result, the best fit point has little relationship to the point which the fake data was generated at.

The two methods are compared for a particular fake data fit in Figure 6.11. The $\Delta \chi^{2}$ method to fit to a data set is:

- For each value of $\Delta m^{2}, 10$ points equally spaced values of $\sin ^{2}(2 \theta)$ are chosen (6 values of $\Delta m^{2}$ shown in Fig. 6.11, 60 points total).

- At each $\Delta m^{2}-\sin ^{2}(2 \theta)$ point, 50 fake data sets used to form a $\Delta \chi^{2}$ distribution.

- The $\Delta \chi^{2}$ distribution of 50 fake experiments is used to calculate $\Delta \chi^{2}(90 \% \mathrm{CL})$, the 


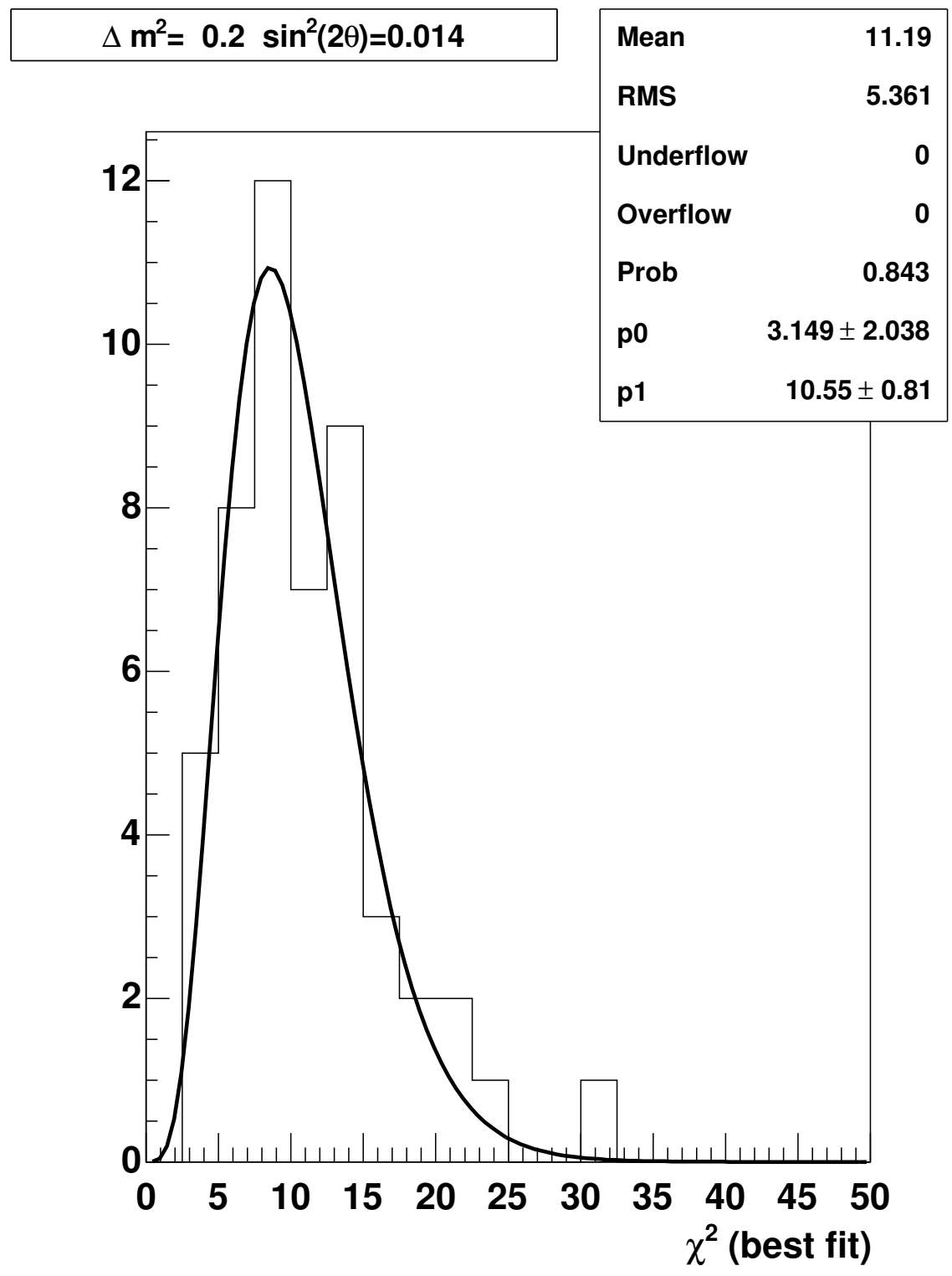

Figure 6.7: The best fit $\chi^{2}$ distribution of 50 fake data experiments with negligible oscillation $\left(\Delta m^{2}=0.2 \mathrm{eV}^{2}, \sin ^{2}(2 \theta)=0.014\right)$. The shape-only error matrix is used to calculate the $\chi^{2}$. The black line shows a fit to a $\chi^{2}$ distribution with $10.6 \pm 0.8 \mathrm{DOF}$, and the mean is $11.2 \pm 0.8$. 


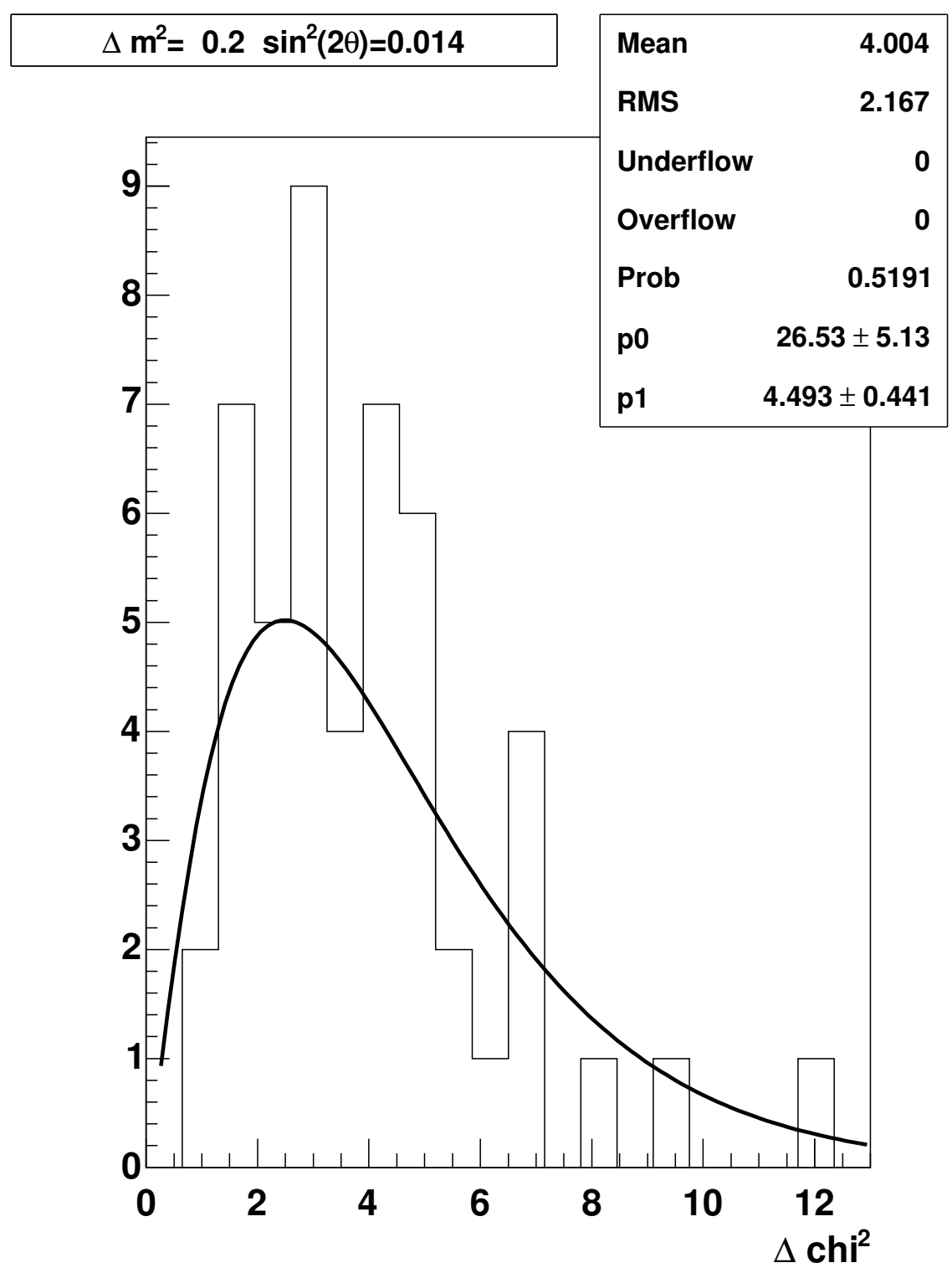

Figure 6.8: The $\Delta \chi^{2}$ distribution of 50 fake data experiments with negligible oscillation $\left(\Delta m^{2}=0.2 \mathrm{eV}^{2}, \sin ^{2}(2 \theta)=0.014\right)$. The shape-only error matrix is used to calculate the $\chi^{2}$. The black line shows a fit to a $\chi^{2}$ distribution with $4.5 \pm 0.4 \mathrm{DOF}$, and the mean is $4.0 \pm 0.3$. 

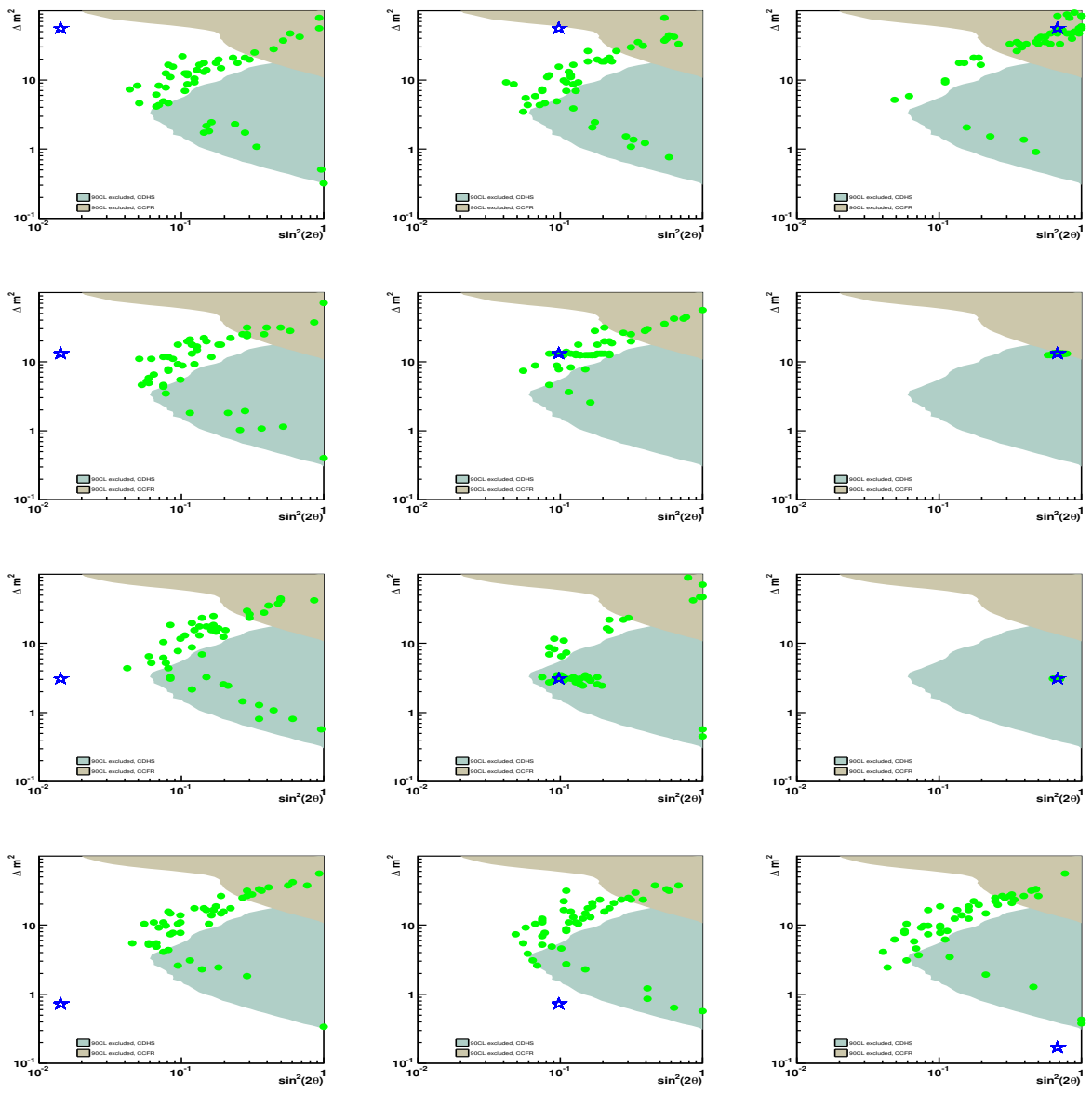

Figure 6.9: The true point (blue star) and the 50 fake experiments best fit (green) points. Top left: True point $=\Delta m^{2}=56 \mathrm{eV}^{2}$, and $\sin ^{2}(2 \theta)=0.014$. Bottom right: True point $=$ $\Delta m^{2}=0.2 \mathrm{eV}^{2}$, and $\sin ^{2}(2 \theta)=0.014$. 


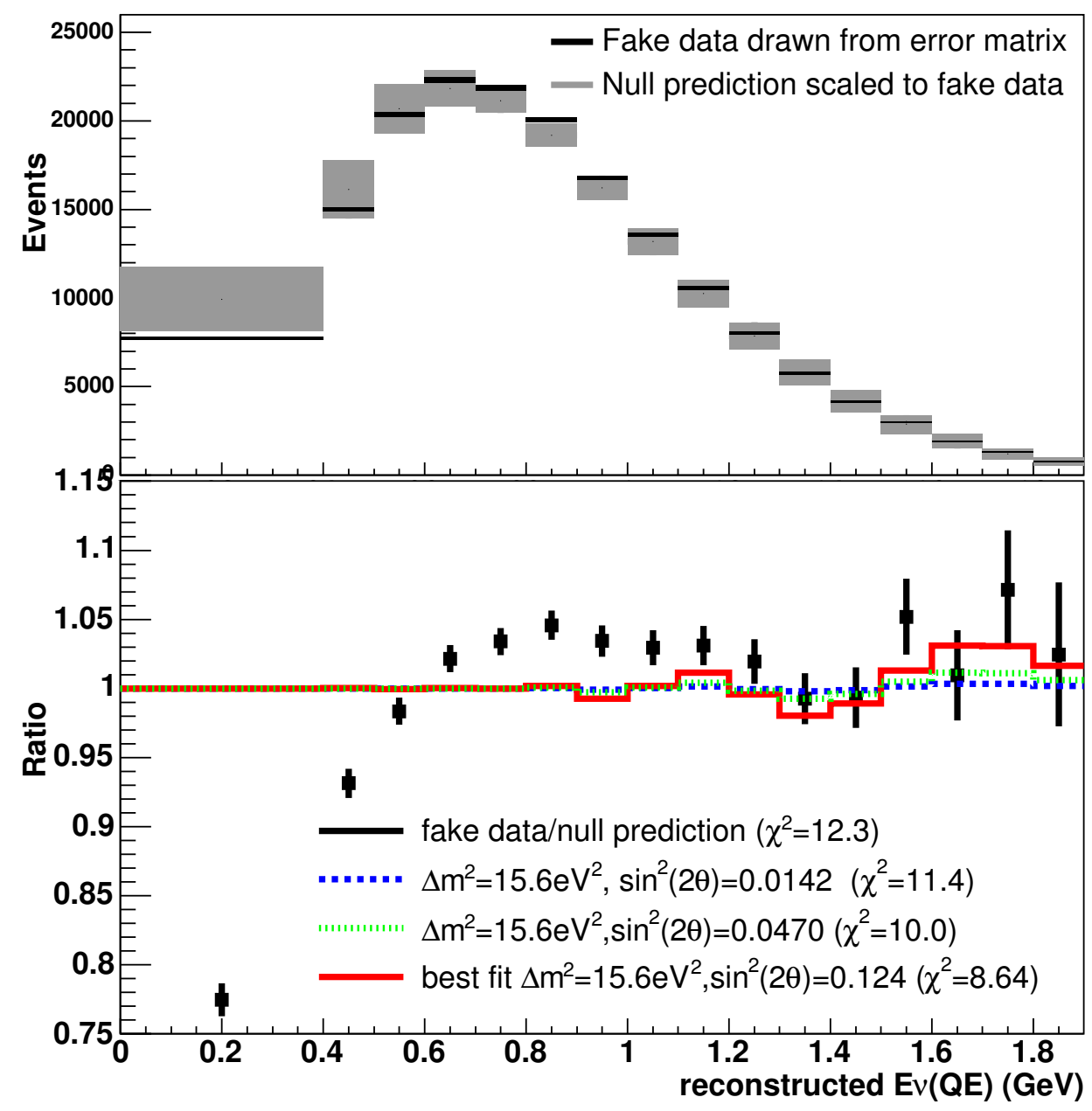

Figure 6.10: Top: A particular fake data drawn with negligible oscillation $\left(\Delta m^{2}=\right.$ $\left.0.2 \mathrm{eV}^{2}, \sin ^{2}(2 \theta)=0.014\right)$ compared to the prediction with no oscillation. The diagonal elements of the shape-only error matrix are shown with the prediction, and the statistical error shown with the fake data. Bottom: The ratio of fake data to null prediction with statistical error bars only. The oscillations corresponding to the best fit point of $\Delta m^{2}=15.6 \mathrm{eV}^{2}, \sin ^{2}(2 \theta)=0.124$ are shown vs. energy bin (red) with $\chi^{2}=8.6$. Two intermediate oscillation scenarios are also shown: $\Delta m^{2}=15.6 \mathrm{eV}^{2}, \sin ^{2}(2 \theta)=0.0142$ (blue) with $\chi^{2}=11.4 \Delta m^{2}=15.6 \mathrm{eV}^{2}, \sin ^{2}(2 \theta)=0.0472$ (green) with $\chi^{2}=10.0$. 
value of $\Delta \chi^{2}$ where $90 \%$ of the fake experiments have $\Delta \chi^{2}<\Delta \chi^{2}(90 \% \mathrm{CL})$.

- The data set is fit to form $\Delta \chi^{2}$ (data).

- The first $\Delta m^{2}-\sin ^{2}(2 \theta)$ point where $\Delta \chi^{2}($ data $)>\Delta \chi^{2}(90 \%$ CL) denotes the allowed 90\%CL region.

The $\Delta \chi^{2}$ method appears to give results consistent with and slightly better than Pearson $\chi^{2}$ method. It mimics the behavior of the sudden changes to the limit (discussed in Section 6.4.3). However, it is computationally untenable. Each point takes approximately 50 minutes on a computing cluster, which is prohibitive except as a cross check. The Pearson $\chi^{2}$ method runs in $\sim 1$ minute for the entire fit.

\subsubsection{Large Variations in Sensitivity Across $\Delta m^{2}$ aka "Wiggles"}

The rapid change in limit curves as a function of $\Delta m^{2}$ for a fixed $\sin ^{2}(2 \theta)$ ("wiggles") are an interesting property of the oscillations in this region. This structure appears in all fake data studies, and is present to a lesser degree in the sensitivity. The source of the wiggles is the coupling between the error envelope and the very different behavior vs. $E_{\nu}^{Q E}$ for $\Delta m^{2}$ close in value. Figure 6.12 shows the fractional difference between oscillation and no oscillation as a function of $E_{\nu}^{Q E}$ for various $\Delta m^{2}$ choices (unoscillated MC-oscillated $\mathrm{MC}) /\left(\right.$ unoscillated MC). As an example of this effect, note that because $\Delta m^{2}=3 \mathrm{eV}^{2}$ induces a larger fractional excursion, and sits outside the error envelope, it gains more $\chi^{2}$, but the nearby value of $\Delta m^{2}=2 \mathrm{eV}^{2}$ sits mostly within the error envelope and has a correspondingly lower $\chi^{2}$. The flat cut on $\chi^{2}>23.5$ for $90 \% \mathrm{CL}$ will then produce $\Delta m^{2}$ regions within the $\chi^{2}$ cut for a fixed $\sin ^{2}(2 \theta)$, and regions of $\Delta m^{2}$ outside of it, i.e. the wiggle structure observed. Figure 6.13 shows the $\chi^{2}(90 \%)$ cut value and the $\chi^{2}$ vs. $\Delta m^{2}$ for two statistically independant fake data throws. The statistical fluctuations change the period and amplitude of the wiggle slightly, so limit curves will appear different based on the statistical fluctuation of the sample. The "frequency" of the wiggles persists despite shifts in binning and changing the number of bins, as shown in Figure 6.14.

The wiggle structure in limit curves also appears in previous disappearance experiments, even with multiple detectors. For example, the limit set by the Bugey experi- 


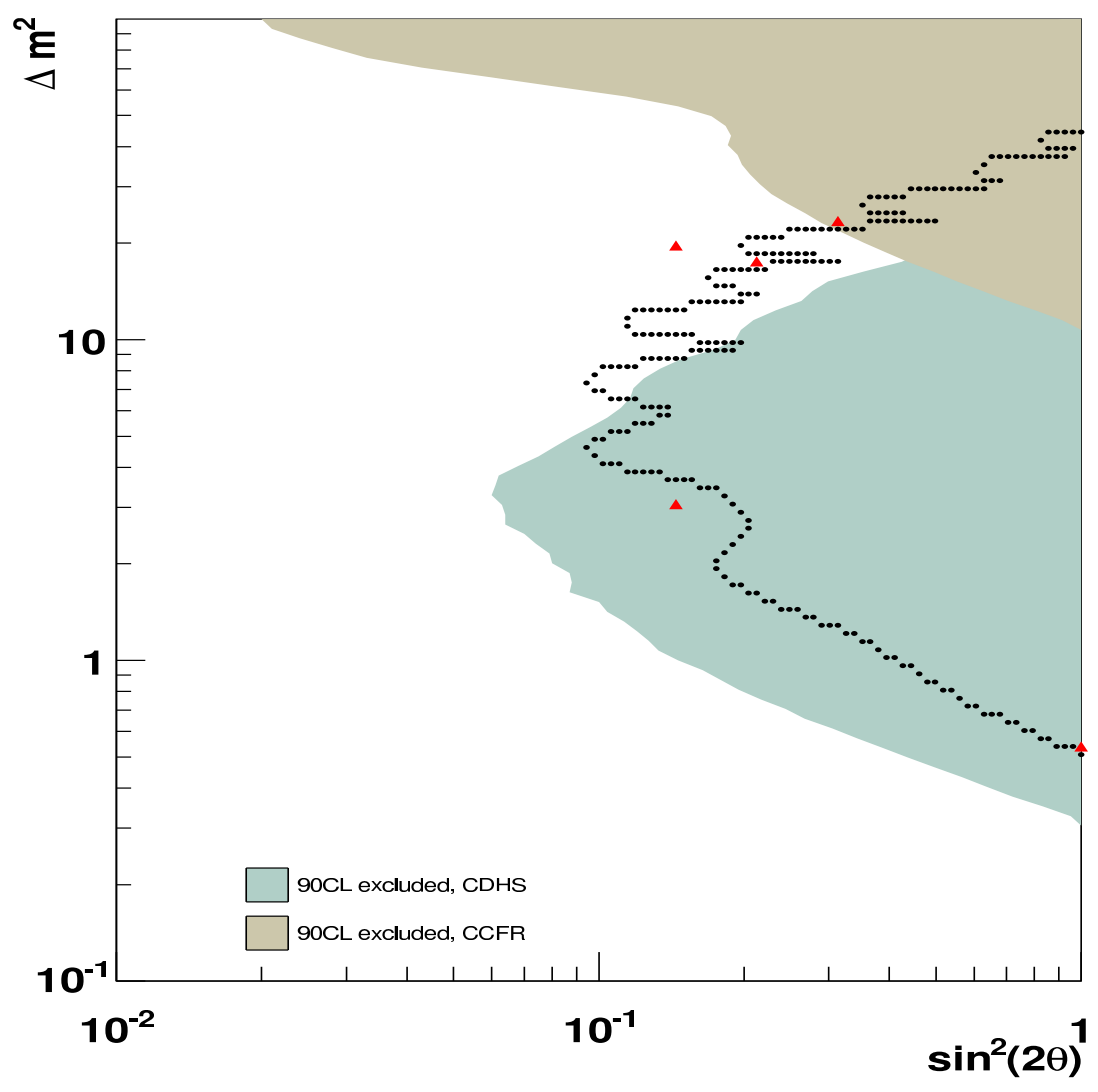

Figure 6.11: Comparison of $90 \%$ CL limits to a fake data set using the $\Delta \chi^{2}$ method (red triangles) and Pearson $\chi^{2}$ method (black circles) with shape-only error matrices. Six $\Delta m^{2}$ points were used in the $\Delta \chi^{2}$ method. 


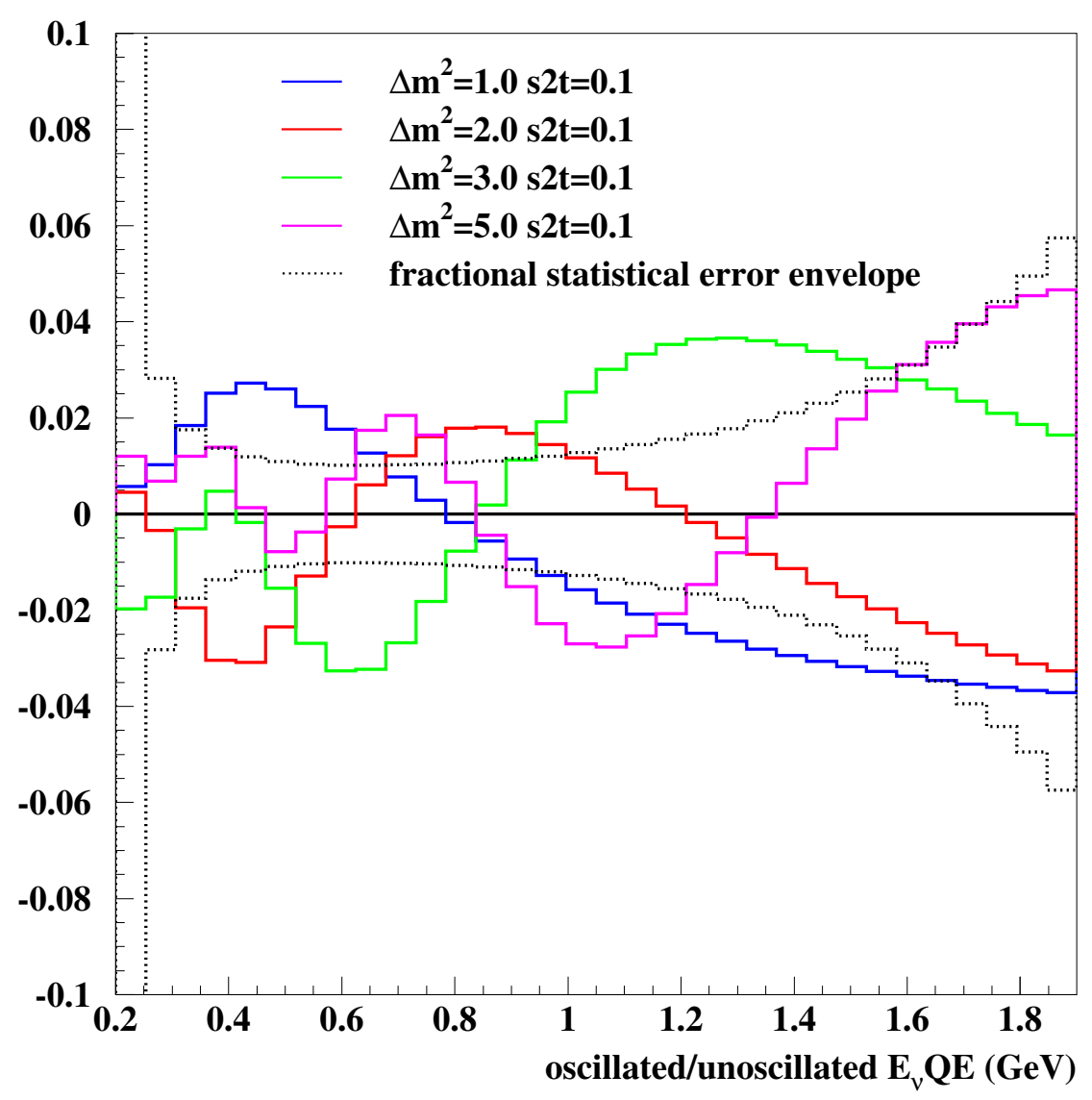

Figure 6.12: Fractional difference between unoscillated prediction (no signal) and oscillated prediction vs. $E_{\nu}^{Q E}$. Oscillation scenarios correspond to $\sin ^{2}(2 \theta)=0.1$, and $\Delta m^{2}=1 \mathrm{eV}^{2}$ (blue), $\Delta m^{2}=2 \mathrm{eV}^{2}$ (red), $\Delta m^{2}=3 \mathrm{eV}^{2}$ (green) and $\Delta m^{2}=5 \mathrm{eV}^{2}$ (pink). The black dashed lines denote the fractional size of the statistical error across bins. 


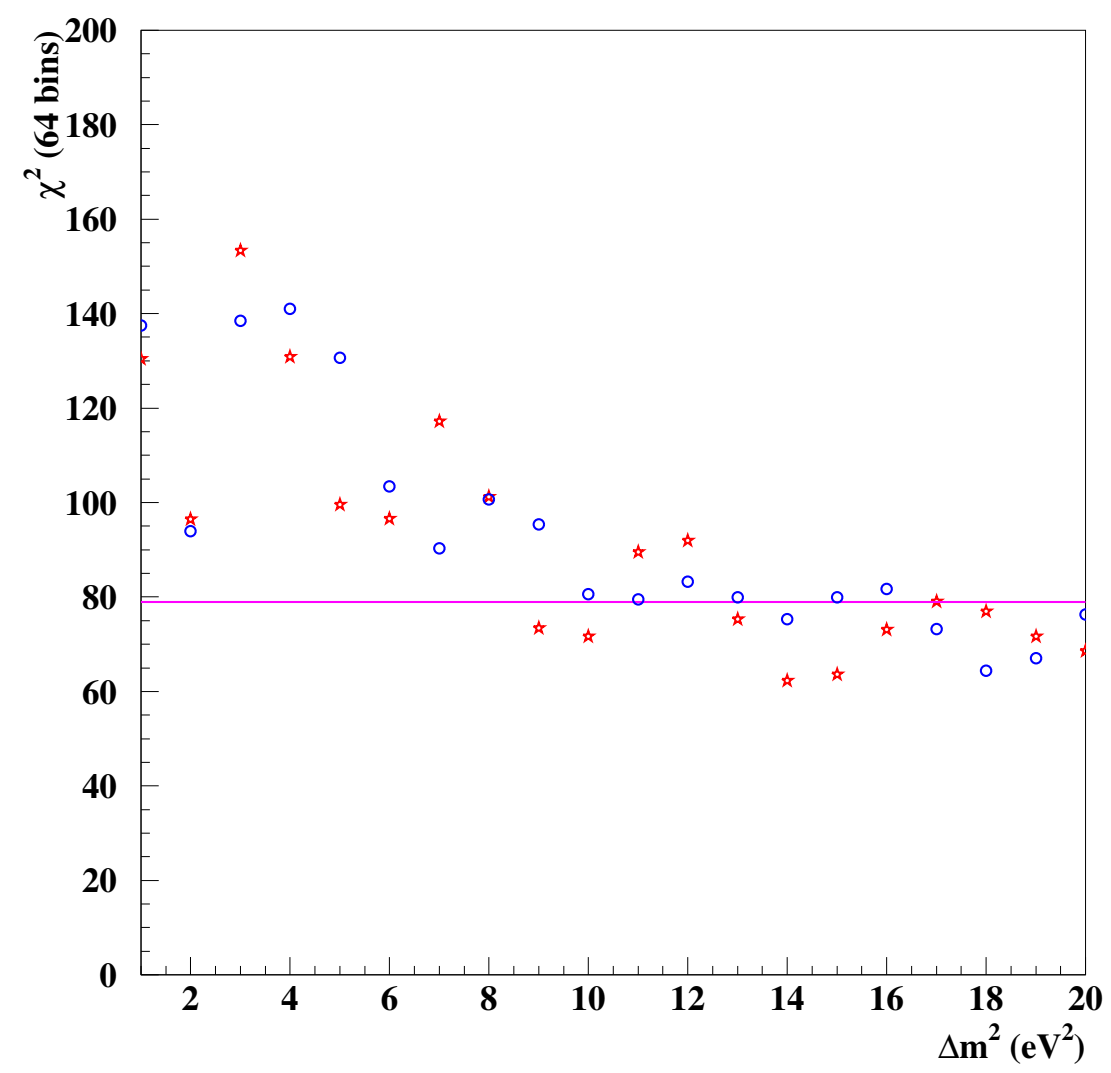

Figure 6.13: $\chi^{2}$ (64 bins), statistical errors only, between fake data and oscillated prediction with $\sin ^{2}(2 \theta)=0.1$ vs. $\Delta m^{2}\left(\mathrm{eV}^{2}\right)$. Two statistically independent fake data drawn from the unoscillated prediction (red star and blue circle) are compared to the $\chi^{2}(90 \% \mathrm{CL})=78.9$ for 64 bins (pink line) 


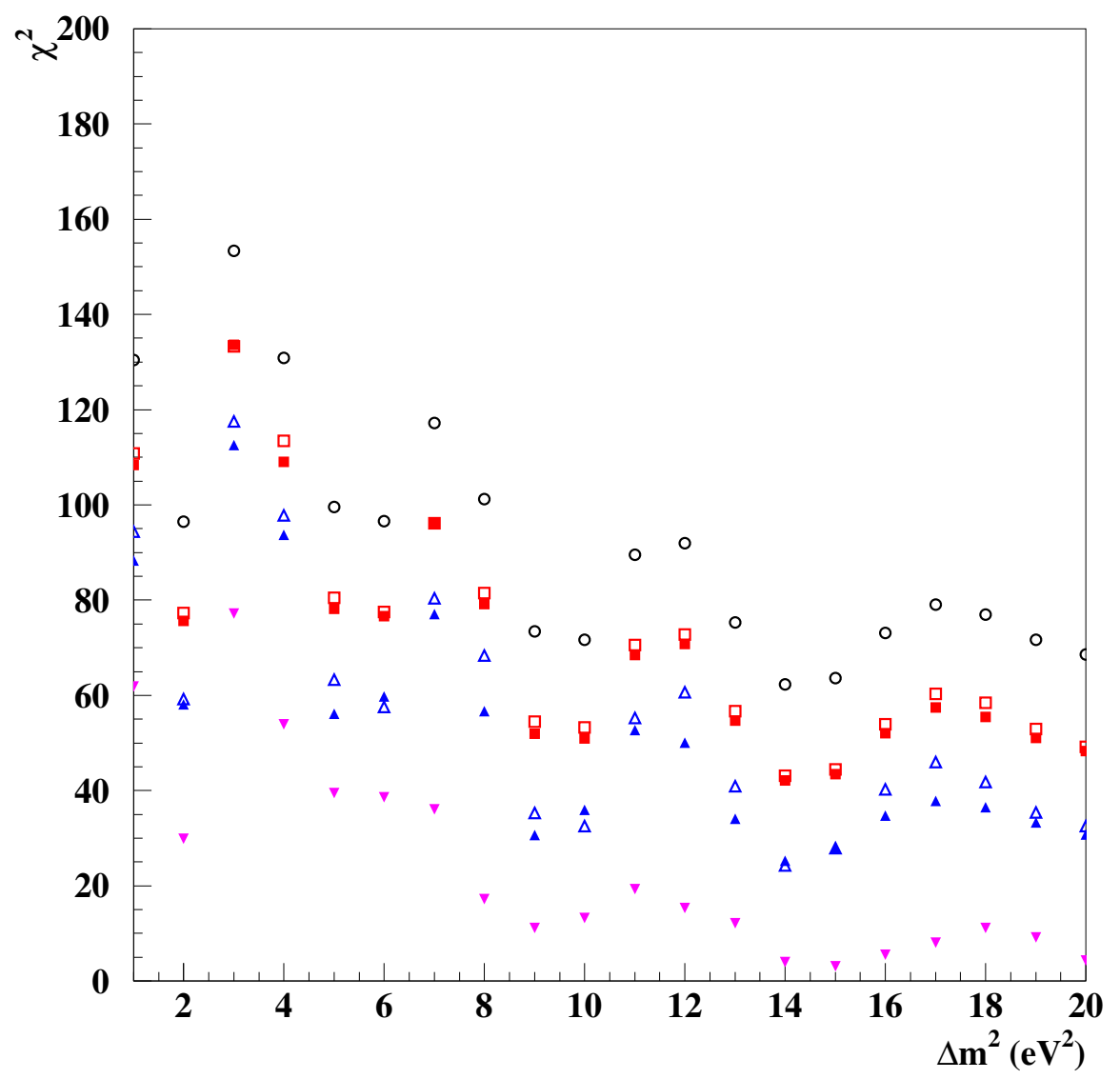

Figure 6.14: $\chi^{2}$, statistical errors only, between data and oscillated MC with $\sin ^{2}(2 \theta)=0.1$ vs. $\Delta m^{2}$ for different binning hypothesis and a particular statistical fake data throw. Open circles 64 bins, open squares are 32 bins, open triangles are 16 bins, and inverted triangles are 8 bins. Shifting the binning by 164 bin and pairing up bins subsequently creates a 33 bin sample, shown in solid squares, and shifting by 364 bins and pairing 4 bins creates a 17 bin sample shown in solid triangles. 


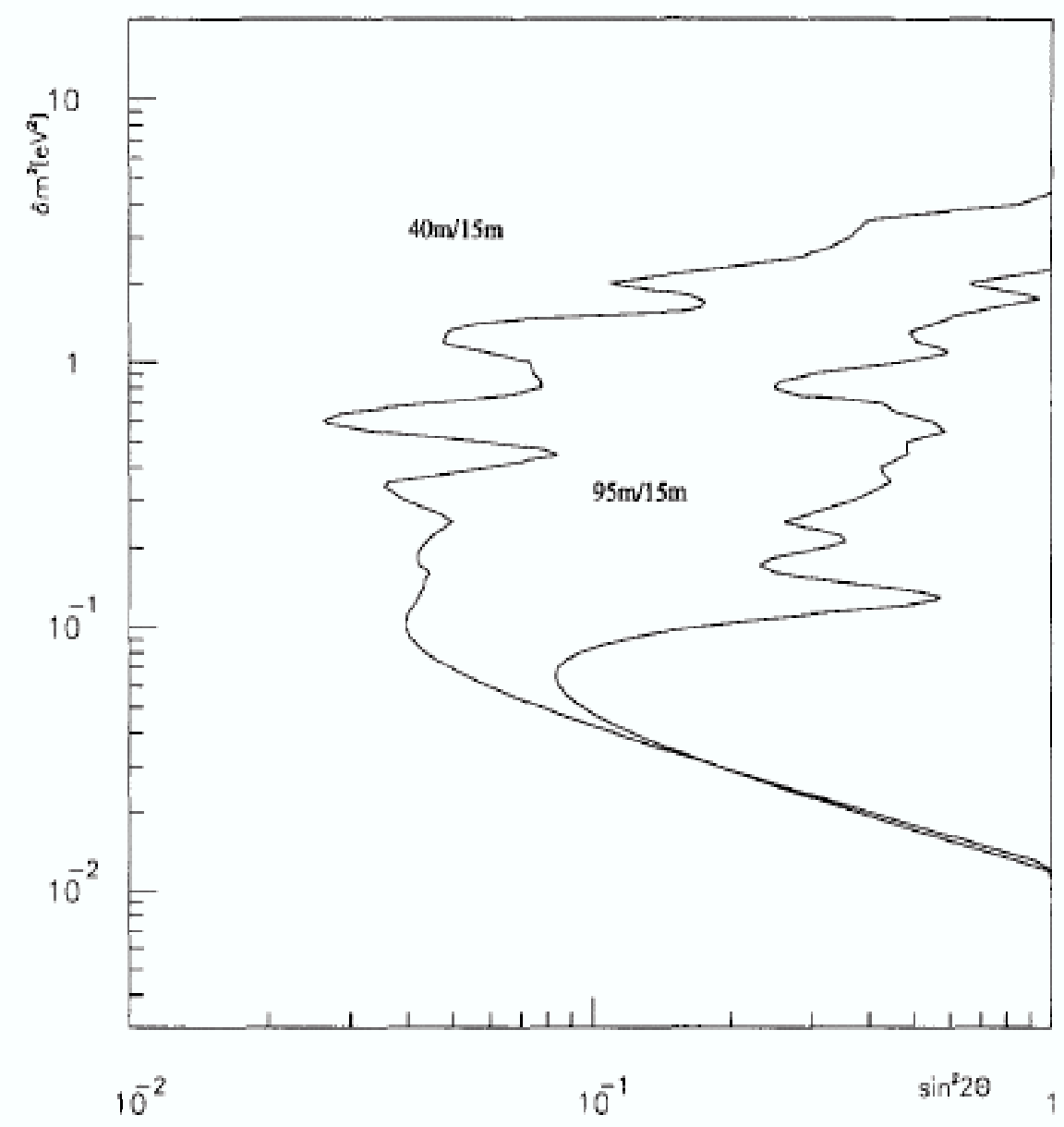

Figure 6.15: $90 \% \mathrm{CL}$ exclusion of $\bar{\nu}_{e}$ disappearance by the Bugey experiment.

ment [116] for $\bar{\nu}_{e}$ disappearance varies substantially over $\Delta m^{2}$ for fixed $\sin ^{2}(2 \theta)$.

\subsection{Results}

The number of events for data (for $\nu$ run Ia with 5.579e20 POT, described in Section 3.5) and prediction (5.579e20) are listed in Table 6.5 and Table 6.6, with the bin delimiters in Table 6.1. The addition of the second two runs of neutrino data( $\nu$ run $\mathrm{Ib}$ and II with $0.882 \mathrm{e} 20$ POT) produces a negligible improvement to the sensitivity, so the $5.579 \mathrm{e} 20$ POT data set is fit for consistency with previous MiniBooNE publications [19], [94].

The top plot of Fig. 6.16 shows the $E_{\nu}^{Q E}$ distribution after selection cuts for the neu- 


\begin{tabular}{|c|c|c|c|c|c|c|c|c|}
\hline bin boundaries & $0 .-0.4$ & $0.4-0.5$ & $0.5-0.6$ & $0.6-0.7$ & $0.7-0.8$ & $0.8-0.9$ & $0.9-1.0$ & $1.0-1.1$ \\
\hline data $\left(5.579 \times 10^{20}\right)$ & 8852. & 16237. & 22150. & 24367. & 23827. & 21895. & 19142. & 14996 \\
\hline $\mathrm{CV}\left(5.579 \times 10^{20}\right)$ & 8329.8 & 13523.3 & 17326.6 & 18288.8 & 17714.1 & 16082.2 & 13603.7 & 11049.2 \\
\hline
\end{tabular}

Table 6.5: MiniBooNE CCQE $\nu_{\mu}$ data $\left(5.579 \times 10^{20}\right)$ and prediction (CV) $\left(5.579 \times 10^{20}\right)$ in energy bins from $0 .-1.1 \mathrm{GeV}$. The prediction is unoscillated and scaled to $5.579 \times 10^{20}$.

\begin{tabular}{|c|c|c|c|c|c|c|c|c|}
\hline bin boundaries & $1.1-1.2$ & $1.2-1.3$ & $1.3-1.4$ & $1.4-1.5$ & $1.5-1.6$ & $1.6-1.7$ & $1.7-1.8$ & $1.8-1.9$ \\
\hline data $\left(5.579 \times 10^{20}\right)$ & 11673. & 8963. & 6348. & 4669. & 3094. & 2070. & 1286. & 885. \\
\hline $\mathrm{CV}\left(5.579 \times 10^{20}\right)$ & 8596.6 & 6587.8 & 4854.6 & 3495.5 & 2403.0 & 1595.5 & 1006.6 & 639.7 \\
\hline
\end{tabular}

Table 6.6: MiniBooNE CCQE $\nu_{\mu}$ data $\left(5.579 \times 10^{20}\right)$ and prediction (CV) for $5.579 \times 10^{20}$ in energy bins from 1.1-1.9GeV. The prediction is unoscillated and scaled to $5.579 \times 10^{20}$.

trino data and the prediction assuming no oscillation (null hypothesis) with diagonal elements of the error matrix. The prediction is relatively normalized to data by a factor of 1.31. The bottom plot shows the ratio of data to null prediction for various oscillation scenarios.

The $\chi^{2}$ between the data and the null hypothesis is 17.78 (16 DOF, 34\% probability) for the neutrino mode sample which is consistent with no oscillations at 90\%CL. The 90\%CL limit set by the MiniBooNE shape-only analysis is shown in Fig. 6.17. The minimum $\chi^{2}=$ $12.72\left(13 \mathrm{DOF}, 47 \%\right.$ probability) at $\Delta m^{2}=17.5 \mathrm{eV}^{2}, \sin ^{2}(2 \theta)=0.16$, where the number of degrees of freedom is estimated from frequentist $\Delta \chi^{2}$ studies mentioned earlier.

\subsubsection{3+2 Oscillation Models}

The region around $\Delta m^{2}=20 \mathrm{eV}^{2}$ where MiniBooNE sets a competitive limit is the favored region of 3+2 models. The LSND experiment oscillation requires a mass splitting of $\Delta m^{2} \sim 1-10 \mathrm{eV}^{2}$, but the CDHS and CCFR experiments exclude much of that region. Where the experimental constraints are weakest, $\Delta m^{2}=20 \mathrm{eV}^{2}$, large values of $\sin ^{2}(2 \theta)$ are still possible, so the allowed region of $3+2$ models populates the region probed by MiniBooNE.

Two particular 3+2 sterile neutrino models are tested against the MiniBooNE energy 


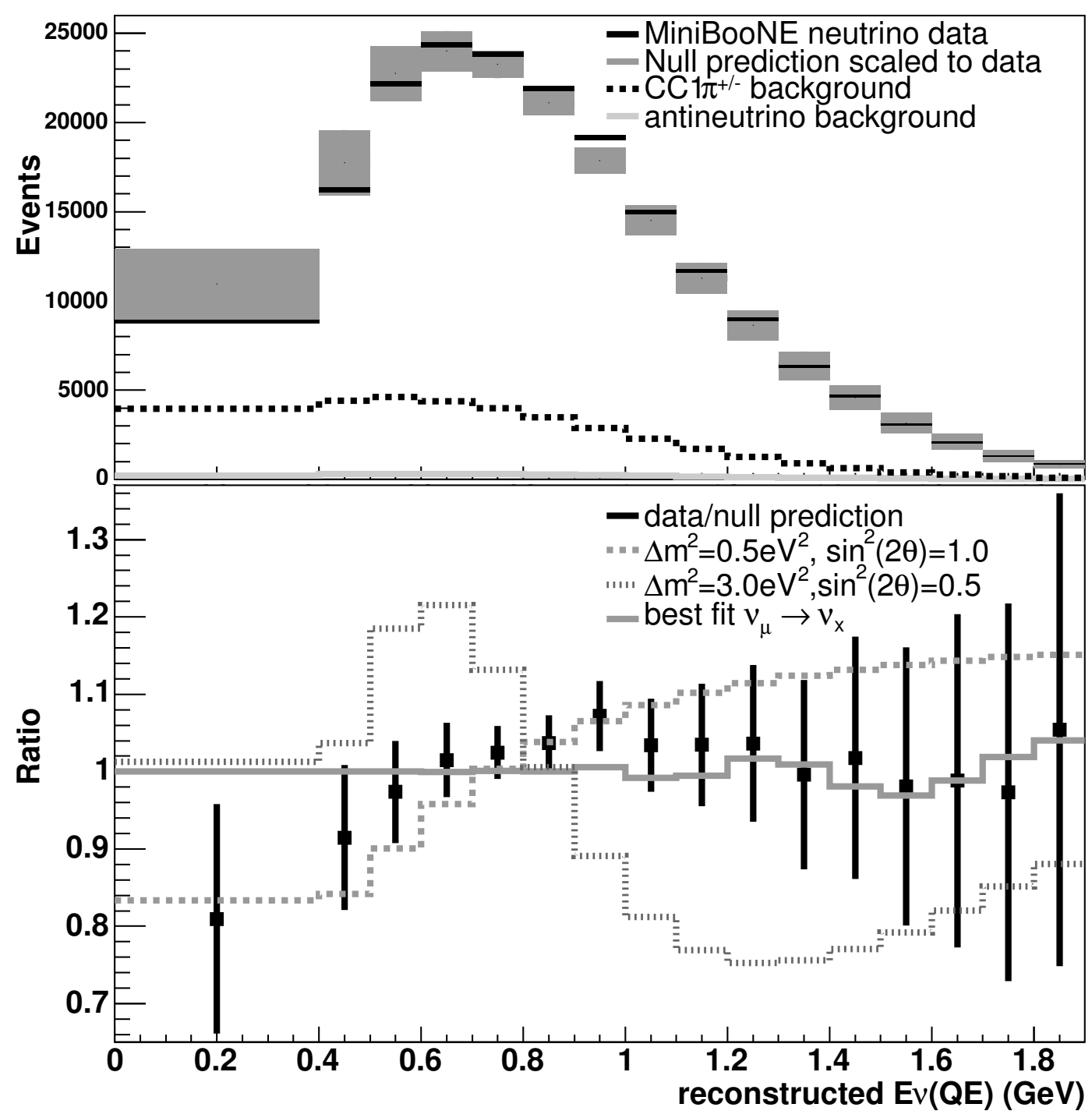

Figure 6.16: The top plot shows the $E_{\nu}^{Q E}$ distribution for neutrino data (black) with statistical error rectangles (thickness of line indicates size of statistical error), and prediction assuming no oscillation (grey). Attached to the prediction are the diagonal elements of the shape error matrix. The predicted CC1 $\pi$ background (dash) and background antineutrino (solid) events are also shown. The bottom plot shows the ratio of data to no oscillation (black), and the ratio of no oscillation to: $\Delta m^{2}=0.5 \mathrm{eV}^{2}, \sin ^{2}(2 \theta)=1.0$ disappearance (dashed line), $\Delta m^{2}=3.0 \mathrm{eV}^{2}, \sin ^{2}(2 \theta)=0.5$ disappearance (dotted line) and for the minimum $\chi^{2}=12.72(13 \mathrm{DOF})$ at $\Delta m^{2}=17.5 \mathrm{eV}^{2}, \sin ^{2}(2 \theta)=0.16$ (solid line). 


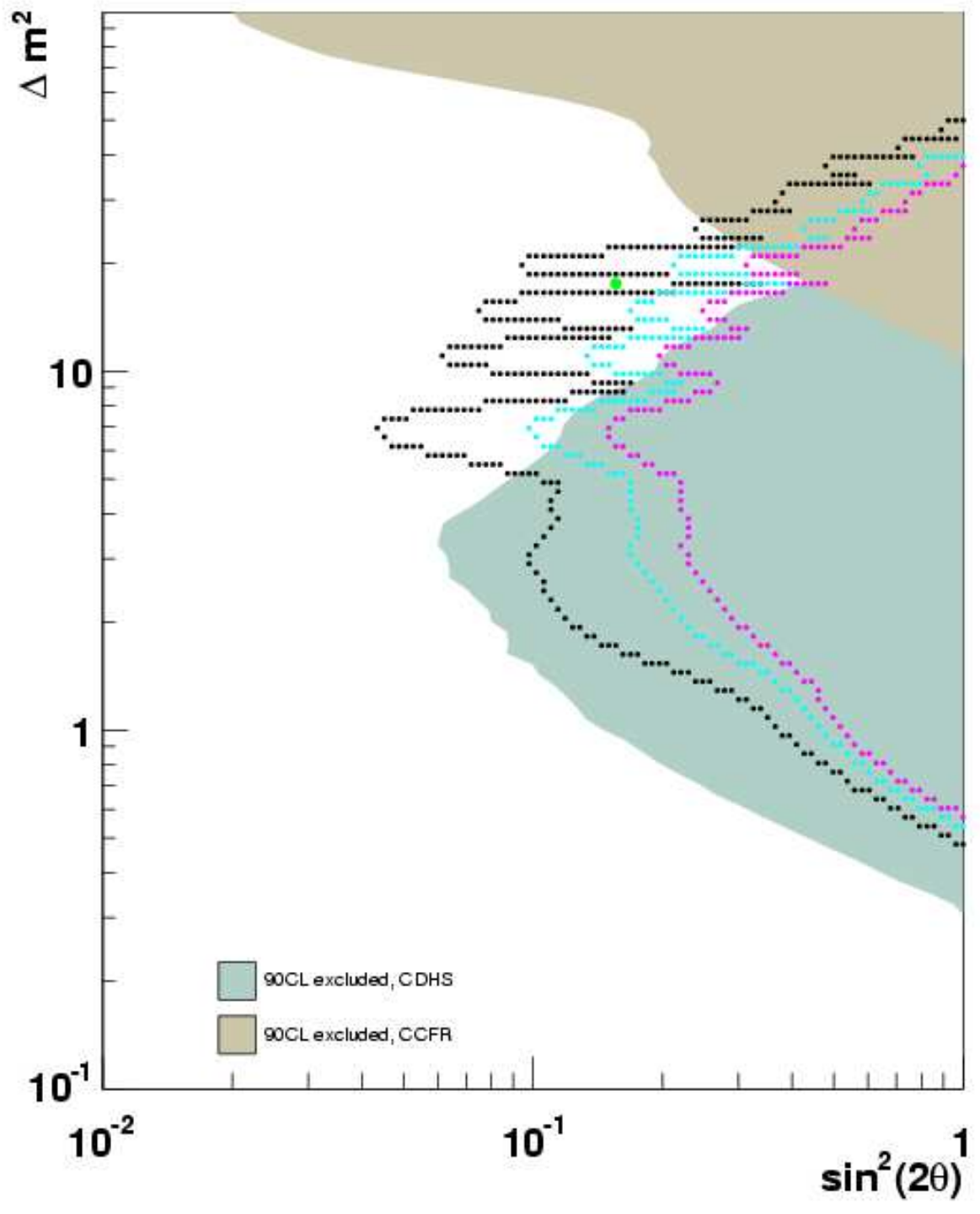

Figure 6.17: Pearson $\chi^{2}$ fit for disappearance using the $5.579 \times 10^{20}$ POT MiniBooNE CCQE $\nu_{\mu}$ sample. A shape-only error matrix is used. The 90\% CL (black) $3 \sigma$ (cyan) and $5 \sigma$ (pink) limits are shown, along with CDHS (light blue) and CCFR (brown) 


\begin{tabular}{|c|c|c|c|c|c|c|}
\hline 3+2 model source & $\Delta m_{41}^{2}\left(\mathrm{eV}^{2}\right)$ & $\Delta m_{51}^{2}\left(\mathrm{eV}^{2}\right)$ & $\left|U_{\mu 4}\right|$ & $\left|U_{\mu 5}\right|$ & $\chi^{2}\left(\nu_{\mu}\right)$ & $\chi^{2}\left(\bar{\nu}_{\mu}\right)$ \\
Ref. [23] & 0.92 & 24. & 0.58 & 0.159 & 18.0 & 10.7 \\
Ref. [25] & 0.89 & 6.49 & 0.16 & 0.12 & 24.7 & 11.4 \\
\hline
\end{tabular}

Table 6.7: The values for $3+2$ sterile neutrino disappearance parameters $\Delta m_{41}^{2}, \Delta m_{51}^{2},\left|U_{\mu 4}\right|^{2}$ and $\left|U_{\mu 5}\right|^{2}$ are listed along with the $\chi^{2}$ (16 DOF) between MiniBooNE $\nu_{\mu}$ and $\bar{\nu}_{\mu}$ data and the model's disappearance prediction in a shape-only fit.

spectrum, as summarized in Table 6.7. The $\chi^{2}$ is calculated between data and prediction with the shape-only error matrix, assuming the values of $3+2$ oscillation described at the best fit to existing data; models where the $\chi^{2}$ between data and prediction is larger than 23.5 are excluded at 90\%CL for 16 bins. The 3+2 sterile neutrino model in Ref. [23] is consistent with the MiniBooNE $\nu_{\mu}$ (and $\bar{\nu}_{\mu}$ data, discussed in the next chapter). The MiniBooNE $\nu_{\mu}$ data rules out Ref. [25] at 90\% CL with $\chi^{2}=24.7(16$ DOF).

MiniBooNE plans to make available the data and prediction points shown in Fig. 6.16 (and Fig. 7.13) so that phenomenologists can incorporate the MiniBooNE disappearance results in their neutrino oscillation studies and fits. 


\section{Chapter 7}

\section{$\bar{\nu}_{\mu}$ Disappearance with MiniBooNE}

The antineutrino analysis uses the same in method and execution as the $\nu_{\mu}$ disappearance analysis. The primary difference between the CCQE $\nu_{\mu}$ sample and CCQE $\bar{\nu}_{\mu}$ analysis is that the latter has a substantial $\nu_{\mu}$ background, as discussed in Section 5.2.5. The result of the $\nu_{\mu}$ analysis, discussed in Chapter 6 , concludes that the $\nu_{\mu}$ do not oscillate for the region of parameter space accessible to MiniBooNE. Consequently, for the $\bar{\nu}_{\mu}$ disappearance search, only $\bar{\nu}_{\mu}$ can oscillate in the disappearance fit, and $\nu_{\mu}$ events will remain fixed.

\subsection{Systematic Uncertainties}

The sources of the systematic uncertainties are the same as considered for the $\nu_{\mu}$ disappearance analysis. The method for producing those uncertainties is also the same. Table 7.1 lists the normalization error for the antineutrino sample, and Figure 7.1 shows the fractional error along the diagonals of the error matrix for each source of error. There are differences at low energy, due to the fact that CCQE $\bar{\nu}_{\mu}$ events interact on hydrogen, and so nuclear effect uncertainties are reduced. In addition, the neutrino events produced by $\pi^{+}$come from low angles not covered by the HARP experiment (Section 5.2.5), and so the flux error is correspondingly larger for those events. The correlations of the shape-only matrix are shown in Fig. 7.2 and look similar to the $\nu_{\mu}$ disappearance analysis matrix. 


\begin{tabular}{|c|c|c|}
\hline uncertainty type & source of uncertainty & normalization uncertainty \\
\hline \multirow[t]{8}{*}{ flux } & & $6.0 \%$ \\
\hline & $\pi^{-}$production & $3.4 \%$ \\
\hline & $\pi^{+}$production & $3.2 \%$ \\
\hline & $K^{+}$production & $0.6 \%$ \\
\hline & $K^{-}$production & $0.1 \%$ \\
\hline & horn magnetic field & $3.0 \%$ \\
\hline & p-Be hadronic cross sections & $1.0 \%$ \\
\hline & POT normalization & $2.0 \%$ \\
\hline \multirow[t]{5}{*}{ cross section } & & $14.0 \%$ \\
\hline & CCQE cross section $\left(M_{A}, \kappa, E_{B}, p_{F}\right)$ & $10.6 \%$ \\
\hline & CCQE cross section model dependence & $6.9 \%$ \\
\hline & CC $\pi$ cross section: $M_{A}^{1 \pi}$ & $5.6 \%$ \\
\hline & $\mathrm{CC} \pi$ cross section model dependence & $2.4 \%$ \\
\hline \multirow[t]{4}{*}{ detector } & & $3.1 \%$ \\
\hline & Optical model + FSI effects & $1.6 \%$ \\
\hline & Electronics model & $2.6 \%$ \\
\hline & Hadronic interactions in detector medium & $0.2 \%$ \\
\hline total & & $15.5 \%$ \\
\hline
\end{tabular}

Table 7.1: Summary of systematic uncertainties considered in the MiniBooNE $\bar{\nu}_{\mu}$ disappearance fit. The third column indicates the normalization uncertainty due to each source of systematic error (Eq.6.9). 


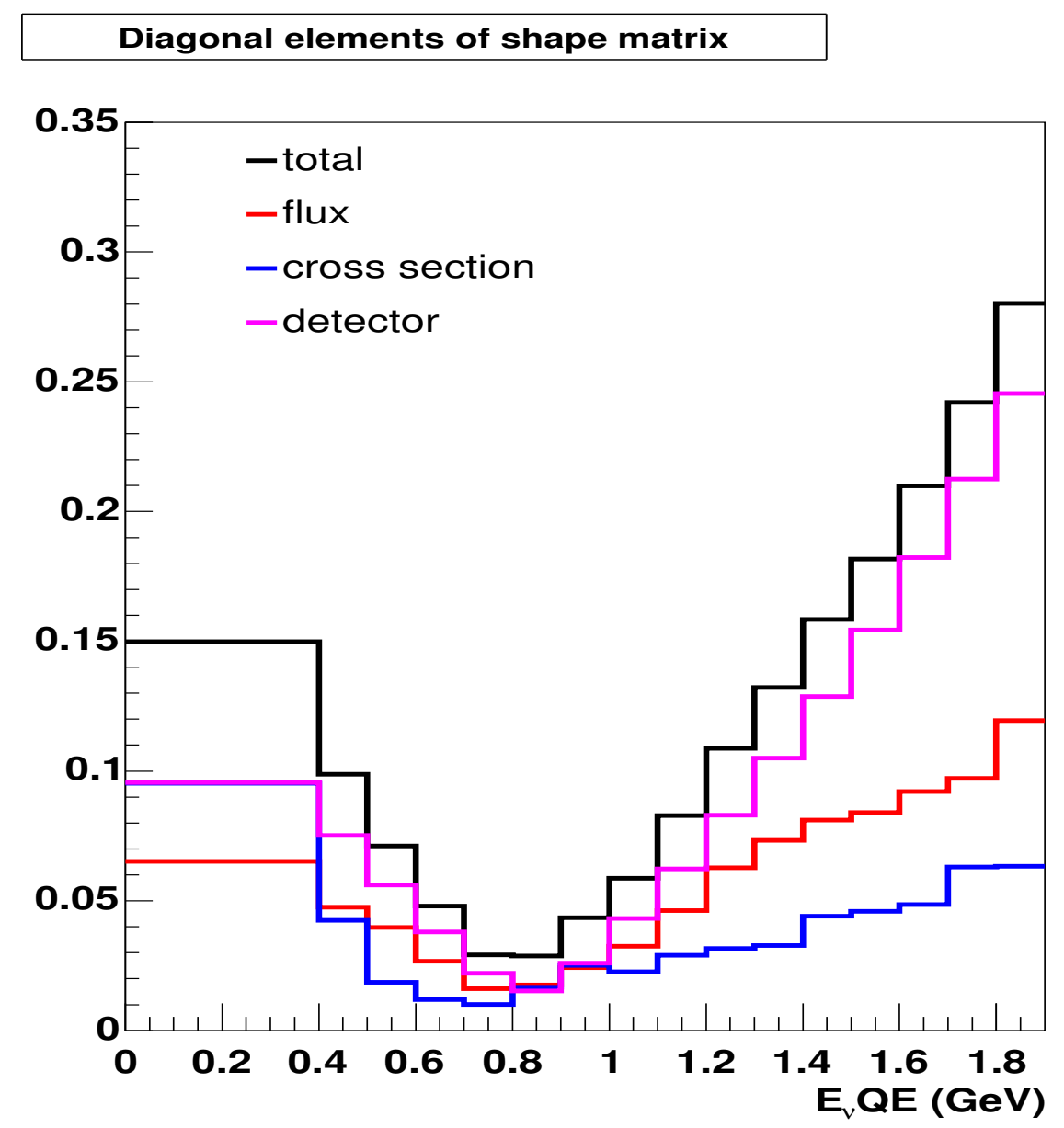

Figure 7.1: Diagonal elements of the shape-only error matrix $\left(\sqrt{M_{i i}^{\text {shape }}} / p_{i i}\right)$ vs. $E_{\nu}^{Q E}$ . The shape-only error matrix is calculated using Eq. 6.10. The total shape-only error matrix (black) is the sum of the flux (red), cross section (blue) and detector (pink) error matrices. 


\section{Correlations of the shape-only error matrix}

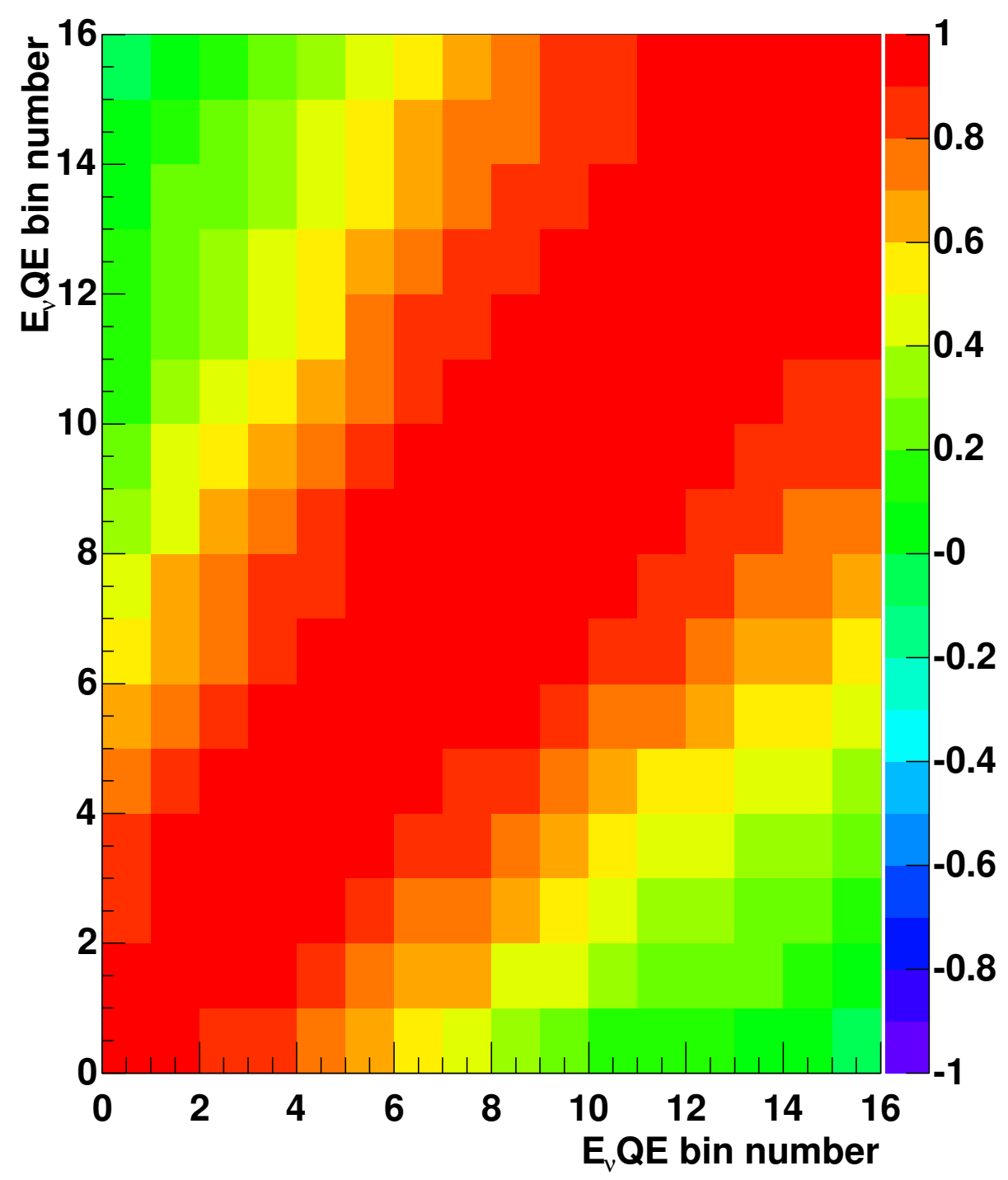

Figure 7.2: The correlation $\rho$ of the total error matrix vs. $E_{\nu}^{Q E}$ bins $(\rho=$ $\left.M_{i j}^{\text {shape }} / \sqrt{\left(M_{i i}^{\text {shape }} M_{j j}^{\text {shape }}\right)}\right)$ for the CCQE $\bar{\nu}_{\mu}$ analysis. The shape-only error matrix is calculated using Eq. 6.10. 

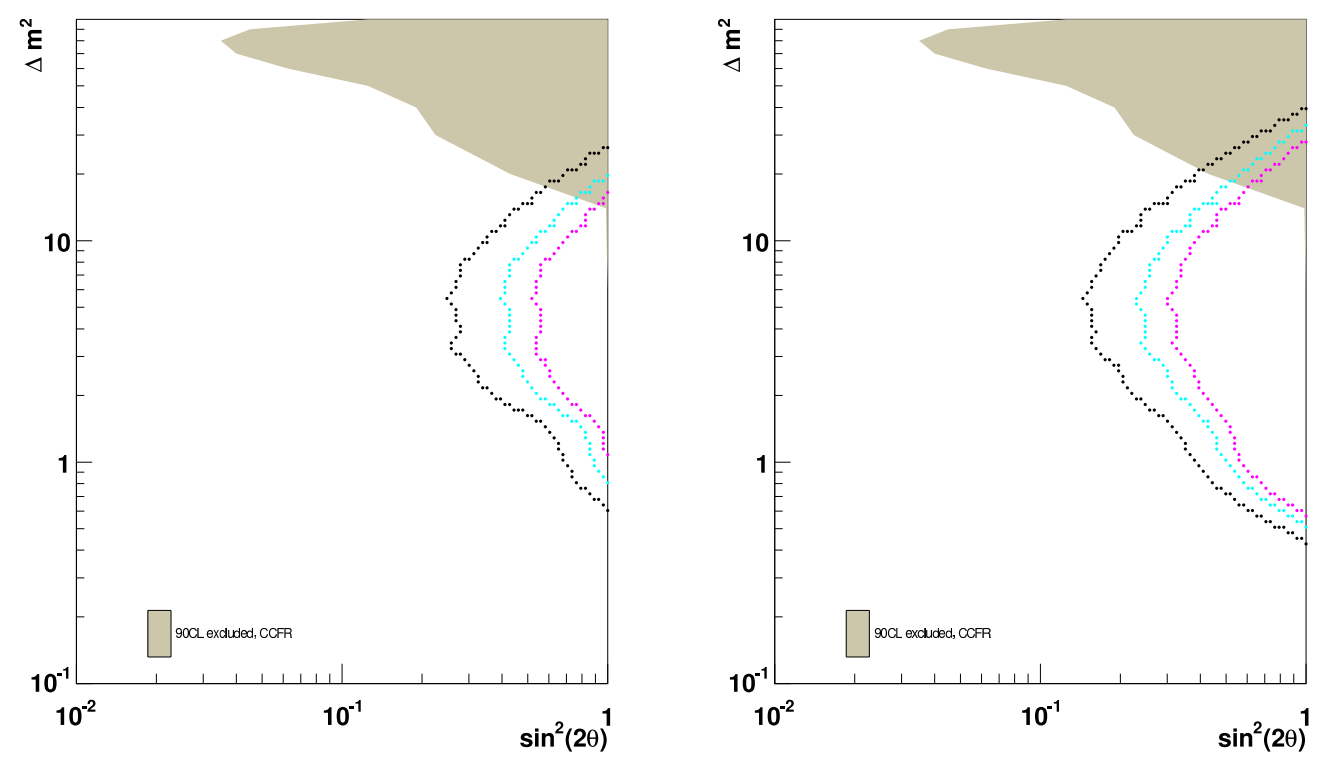

Figure 7.3: Pearson $\chi^{2}$ method 90\% CL sensitivity (black) $3 \sigma$ CL sensitivity (cyan) and $5 \sigma$ CL sensitivity (magenta) for the CCQE $\bar{\nu}_{\mu}$ disappearance analysis, where only antineutrinos are allowed to oscillate (neutrinos are fixed). The 90\% CL exclusion regions for CCFR (light brown) is also shown. Left: Only antineutrinos are allowed to oscillate, and neutrino events are fixed. Right: Both neutrino and antineutrinos oscillate with the same probability (not used in this analysis).

\subsection{Fit Method}

Like the $\nu_{\mu}$ disappearance analysis, a Pearson's $\chi^{2}$ fit is performed. As the $\nu_{\mu}$ disappearance analysis excludes the region which the $\bar{\nu}_{\mu}$ disappearance covers, the neutrino events are held fixed and only the antineutrino events can oscillate. This method determines the limit on a model where the $\bar{\nu}_{\mu}$ can oscillate but the $\nu_{\mu}$ cannot. The sensitivity to antineutrino disappearance for MiniBooNE is shown in Fig. 7.3. If the neutrino events can also oscillate, the MiniBooNE $\bar{\nu}_{\mu}$ disappearance analysis sensitivity is comparable with an appropriately POT scaled limit of the $\nu_{\mu}$ disappearance, as shown in Fig. 7.3.

The effect of removing each error is shown in Fig. 7.4. The two most dominant er- 


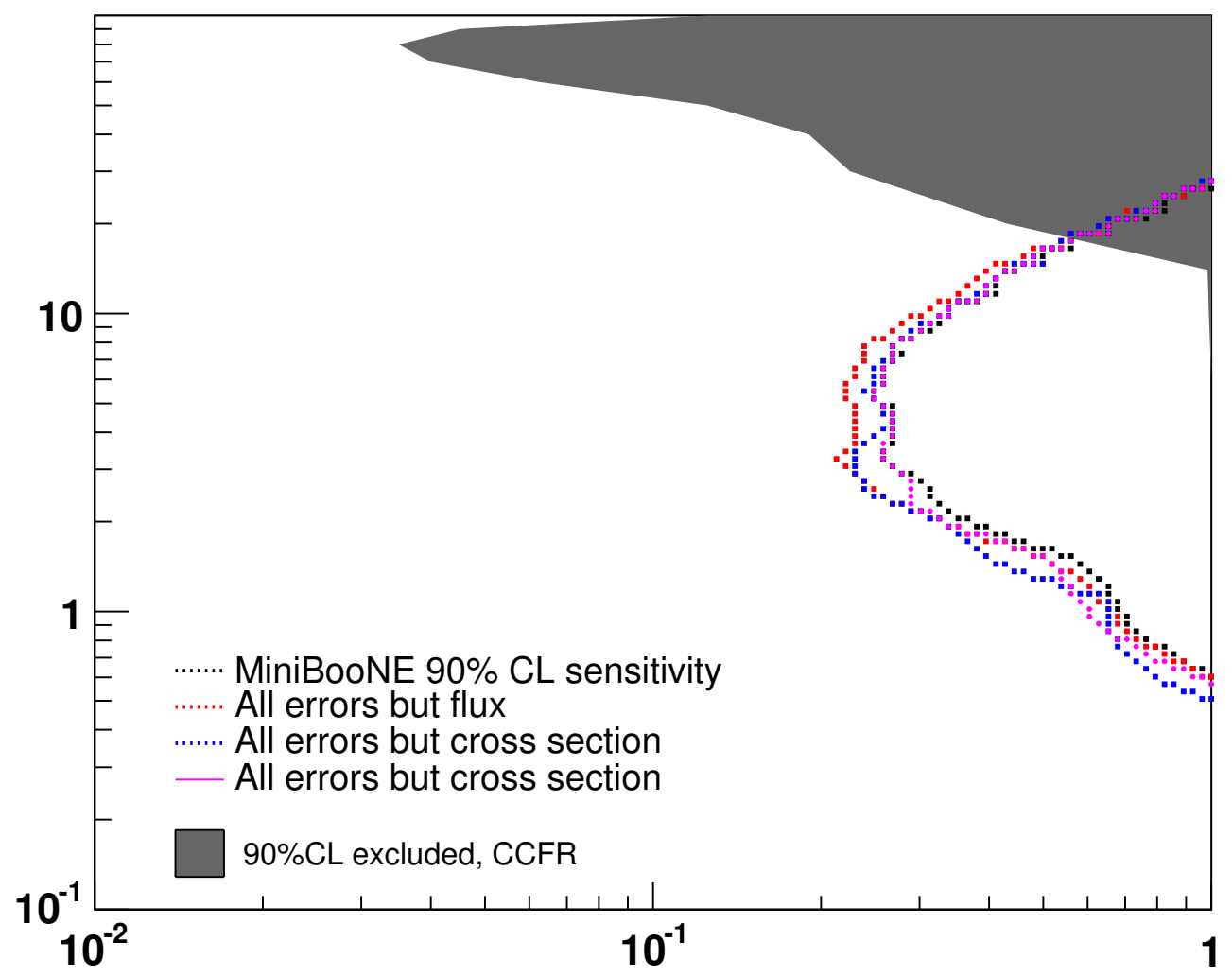

Figure 7.4: Pearson $\chi^{2}$ method $90 \%$ CL sensitivities for the MiniBooNE $\bar{\nu}_{\mu}$ analysis. All errors included is the $90 \%$ CL sensitivty (black). Each error contribution is removed from the total and replotted to demonstrate the effect of the error. All but flux uncertainties (red), cross sections (blue), and detector model (pink) are shown. The 90\% CL exclusion regions for CCFR (dark grey) is also shown. 
rors, like the neutrino disappearance analysis, are flux (parent pion production) and cross section uncertainties.

As this fit is a shape-only fit, the effect of a relative normalization difference between neutrino and antineutrino events must be considered. Let the normalization for events from a $\pi^{+}$(neutrinos), be $\mathrm{N} \pi^{+}$, and normalization from events from a $\pi^{-}$(antineutrinos) be $\mathrm{N} \pi^{-}$. The effect of varying $\mathrm{N} \pi^{+}$and $\mathrm{N} \pi^{-}$is shown in Figure 7.5. The shape change across $E_{\nu}^{Q E}$ is small. To test whether the fit is sensitive to such a change, Fig. 7.6 shows the $\bar{\nu}_{\mu} 90 \%$ CL sensitivity compared to a fit to fake data corresponding to $\mathrm{N} \pi^{+}=0.9, \mathrm{~N} \pi^{-}=1.22$, scaled to the same total number of events, to have as close to the same statistical error as possible. The different $\mathrm{N} \pi$ values have no large effect on the sensitivity or the $\chi^{2}$ surface because the variation is spanned by the current uncertainty on the flux for $\pi^{+}$and $\pi^{-}$ production.

Over the period of $\bar{\nu}_{\mu}$ data-taking, two absorber plates fell into the beamline (see Section 3.2). The effect of these plates is accounted for in the prediction by using three beam simulations, each with the appropriate number of of absorber plates deployed, and so no additional systematic uncertainty is included. The absorber plates change the energy spectrum, as shown in Fig. 7.7, reducing the rate of higher energy events.

\subsection{Results}

For the antinuetrino data set, $3.386 \times 10^{20}$ POT has been processed with three different configurations of absorber planes. Table 7.2 and Table 7.3 give the number of events in data for each absorber configuration, along with the prediction for no absorbers and all absorbers, with the bin delimiters in Table 6.1. Figures 7.9, 7.10 and 7.11 show the relatively normalized data with the prediction under the appropriate absorber reweighting. Figure 7.12 shows the ratio of data to appropriate prediction for each of the different absorbers. The data collected with no absorber in the beamline is consistent between $\bar{\nu}$ run Ia, $\bar{\nu}$ run II, and $\bar{\nu}$ run III, defined in Section 3.5 as shown in Figure 7.8.

The top plot of Fig. 7.13 shows $E_{\nu}^{Q E}$ after selection cuts for the antineutrino data and the prediction assuming no oscillation (null hypothesis) with diagonal elements of the 


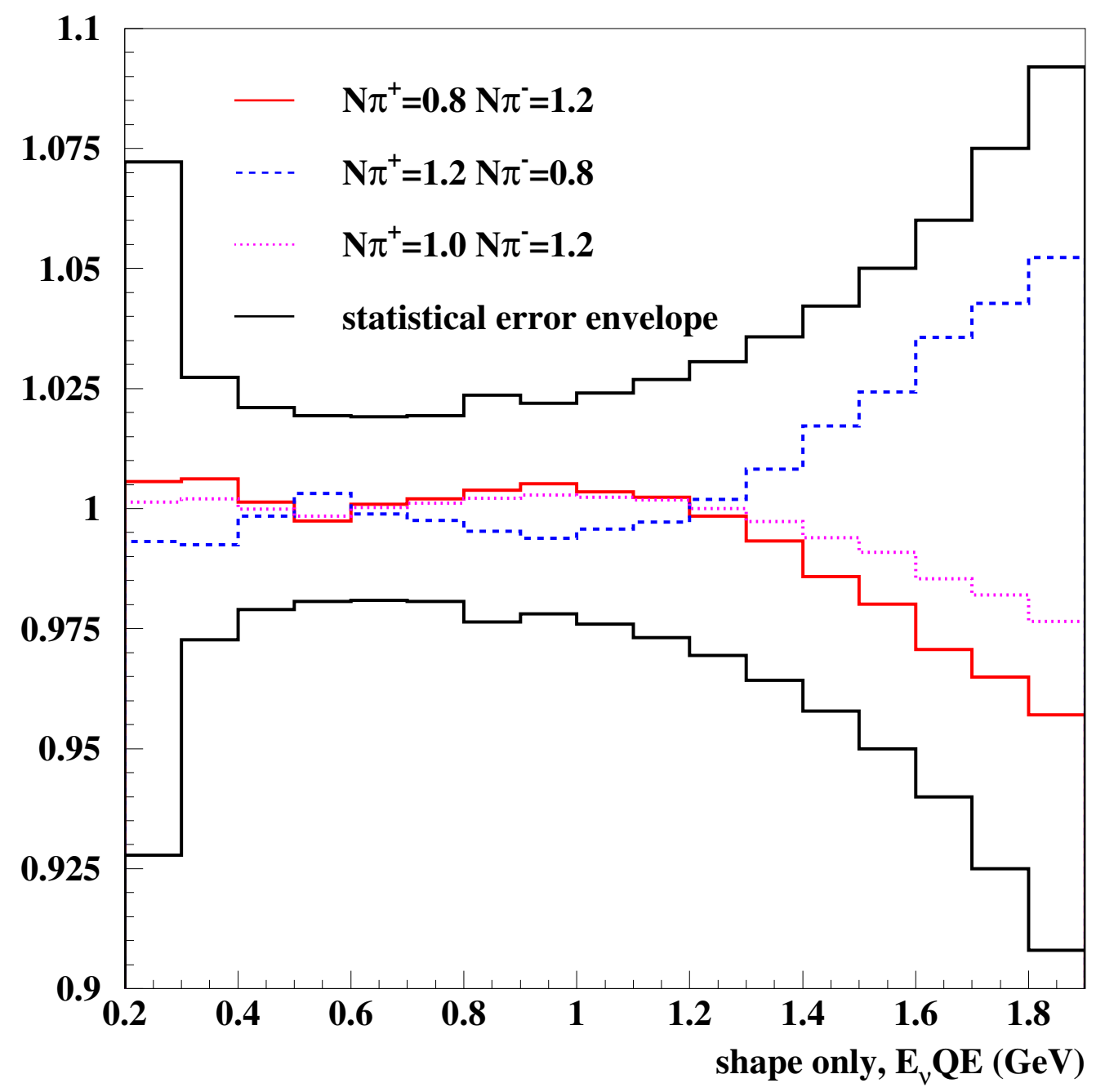

Figure 7.5: The ratio of changed $\mathrm{N} \pi^{+/-}$to unoscillated $\mathrm{N} \pi^{+/-}=1.0$ prediction vs. $E_{\nu}^{Q E}$. The prediction after $N \pi^{+/-}$scaling is applied is renormalized to the total $\mathrm{N} \pi^{+/-}=1.0$ prediction to produce only shape variations. The black solid curves indicate the size of the statistical error on the CCQE $\bar{\nu}_{\mu}$ sample. $\mathrm{N} \pi^{+}=0.8, \mathrm{~N} \pi^{-}=1.2$ (red), $\mathrm{N} \pi^{+}=1.2$, $\mathrm{N} \pi^{-}=0.8$ (blue dash), and $\mathrm{N} \pi^{+}=1.0, \mathrm{~N} \pi^{-}=1.2$ (pink dot) are compared to $\mathrm{N} \pi+/-=$ 1.0. 


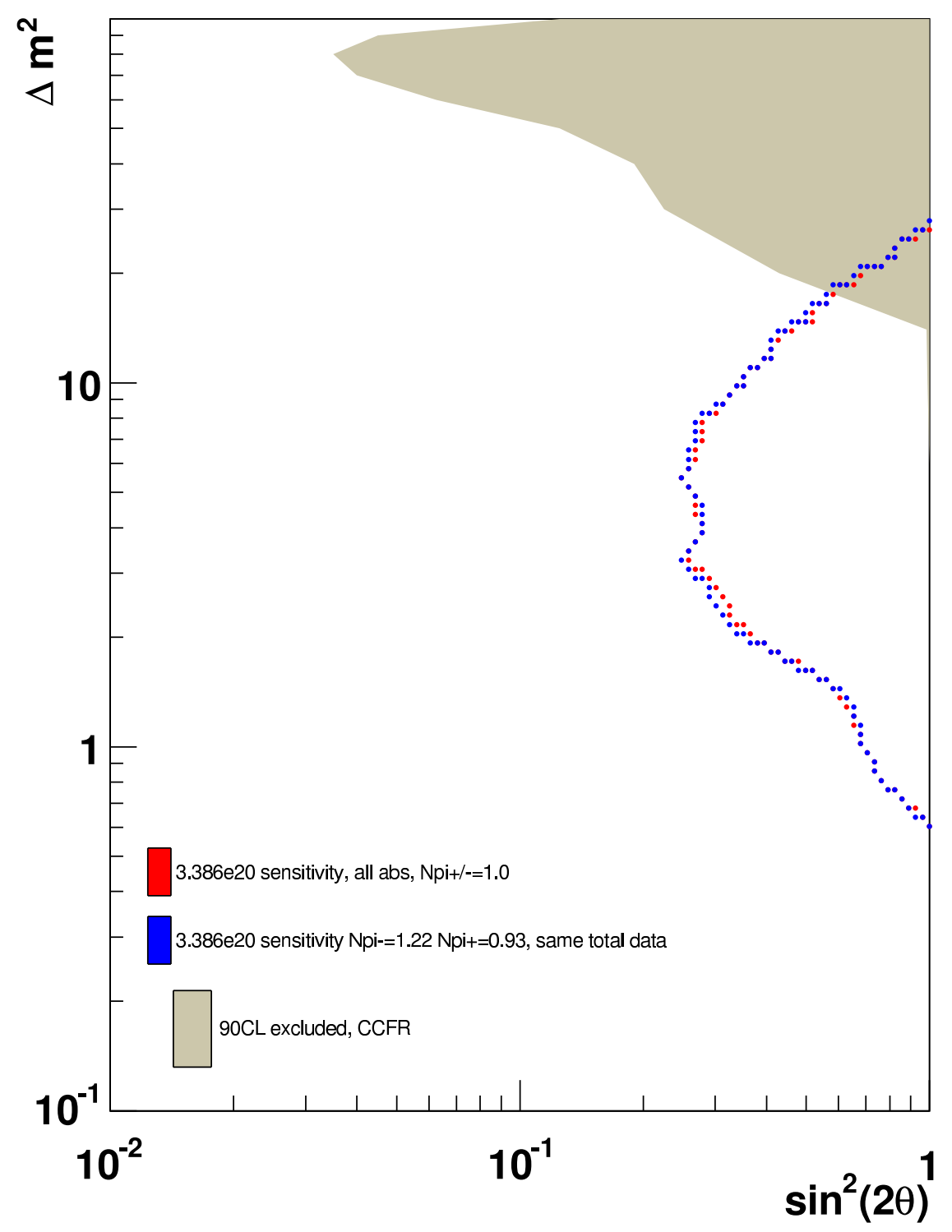

Figure 7.6: Pearson $\chi^{2}$ method 90\% CL fit for the CCQE $\bar{\nu}_{\mu}$ (red) analysis with $\mathrm{N} \pi^{+/-}=1.0$ (fit to prediction with the same underlying prediction) compared to a fit to a prediction with $\mathrm{N} \pi^{+}=0.9, \mathrm{~N} \pi^{-}=1.22$, scaled to same total data as the $\mathrm{N} \pi^{+/-}=1.0$ fit (blue). Only $\bar{\nu}_{\mu}$ are allowed to oscillate. The 90\% CL exclusion regions for CCFR (light brown) are shown. 


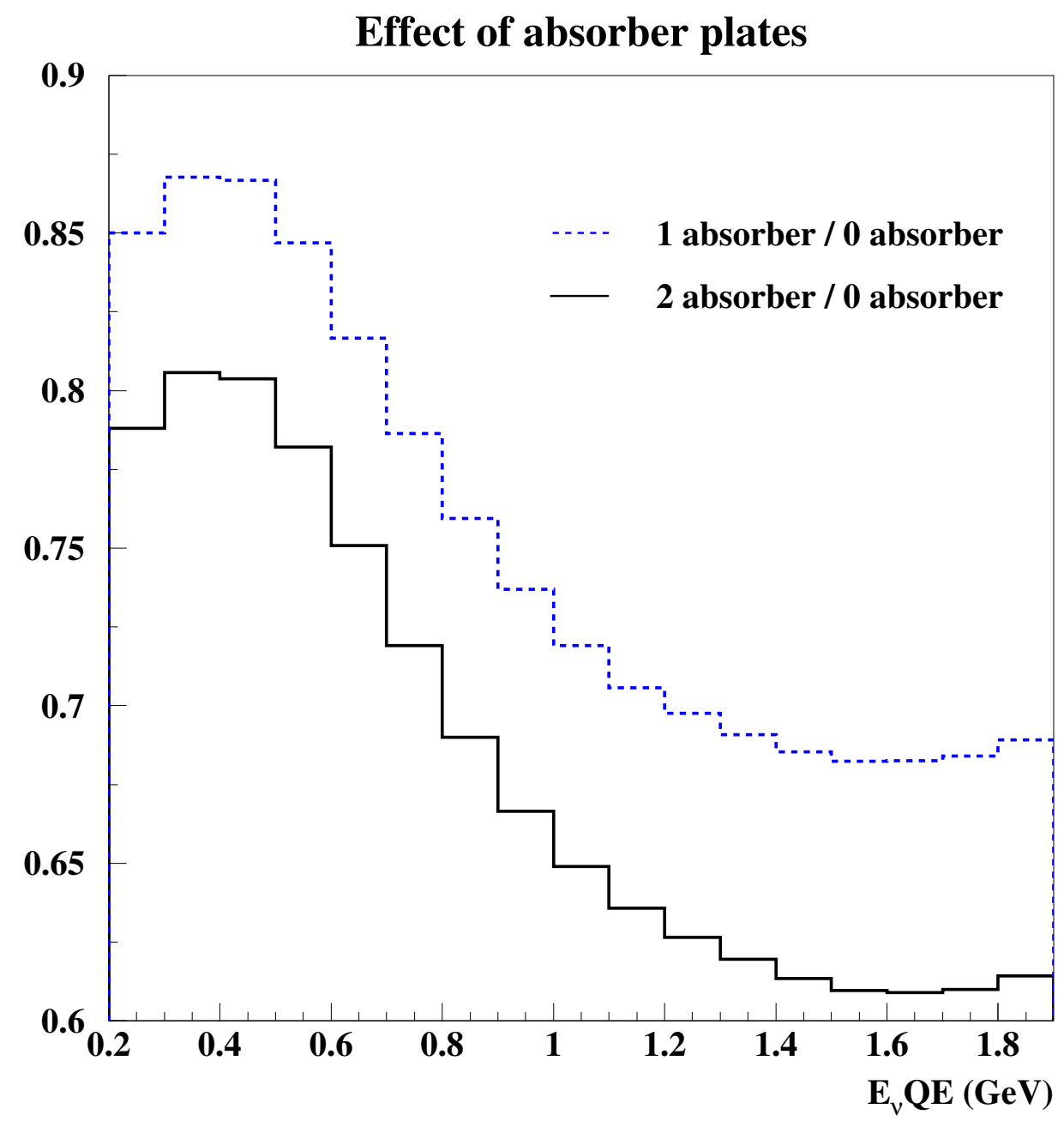

Figure 7.7: Effect of absorber plates on $E_{\nu}^{Q E}$. The ratio of one (two) absorber to no absorbers is shown with a blue dashed line (solid black line) as a function of $E_{\nu}^{Q E}$. 


\section{CCQE $\bar{v}$ sample for 0 absorber data}

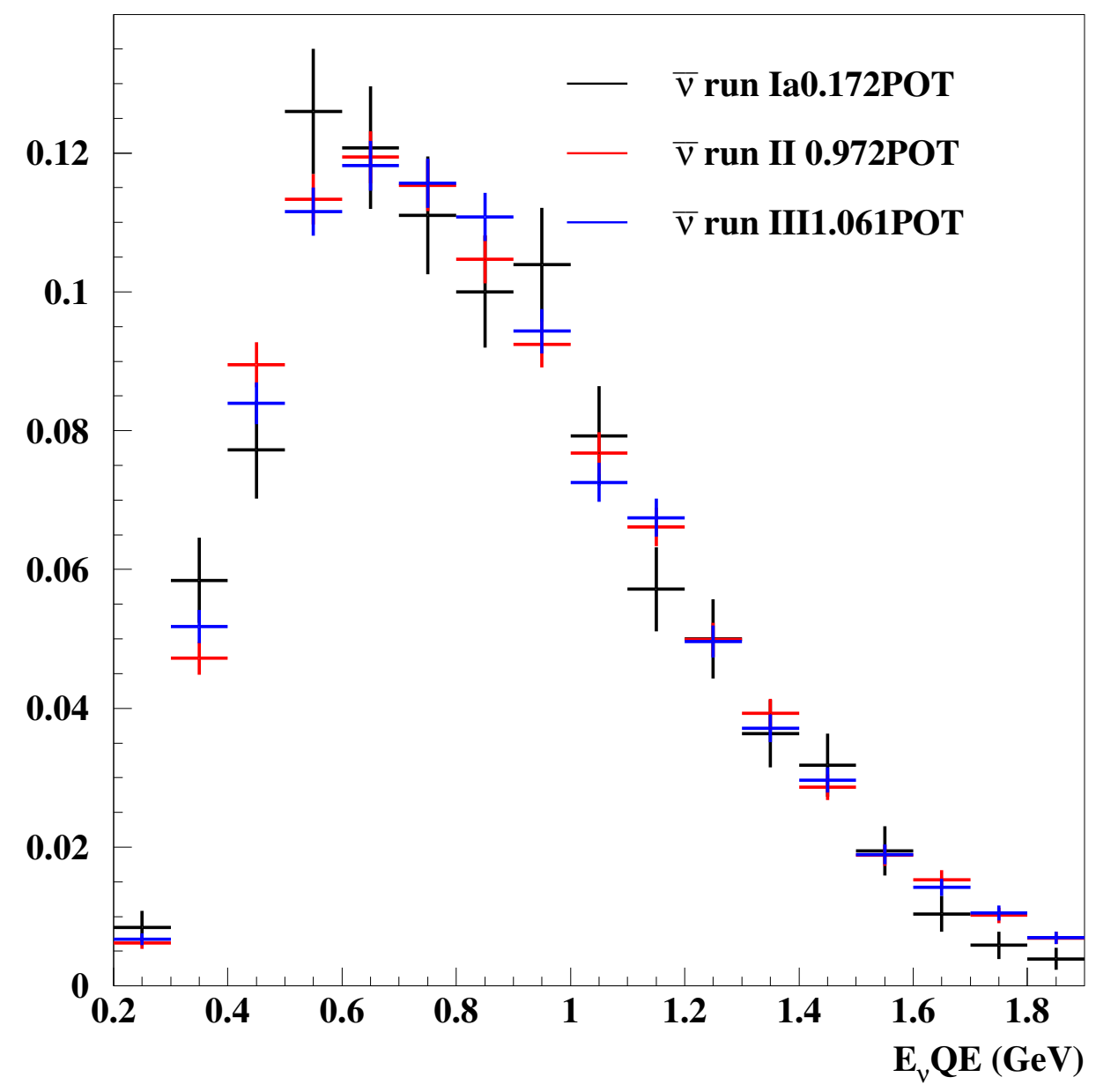

Figure 7.8: Data vs. $E_{\nu}^{Q E}$ for three periods in time, $\bar{\nu}$ run Ia, $\bar{\nu}$ run II, and $\bar{\nu}$ run III, defined in Section 3.5, when no absorber plates were deployed in the beamline. All distributions are scaled to unit area to better compare shape, and are plotted with statistical errors only. The first period of 0 absorber running is shown in black and corresponds to $0.172 \times 10^{20}$ POT. The second period of 0 absorber running is shown in red and corresponds to $0.972 \times 10^{20} \mathrm{POT}$. The third period of 0 absorber running is shown in blue and corresponds to $1.061 \times 10^{20} \mathrm{POT}$. 


\section{data (w/stat error) and MC, 0absorber 2.201POT}

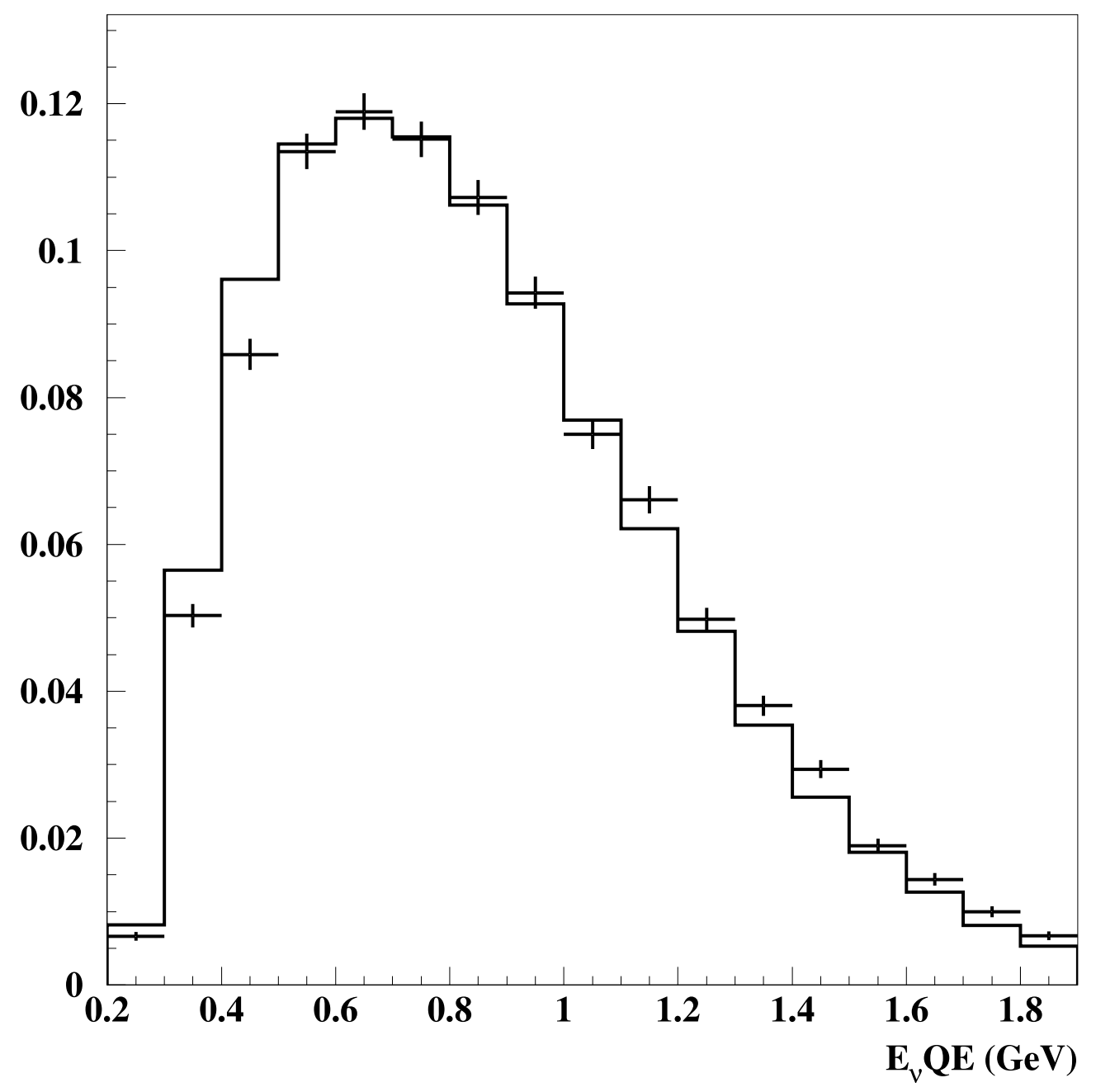

Figure 7.9: Data (error bars) and unoscillated prediction $E_{\nu}^{Q E}$ for the 0 absorber configuration, with unit normalization to better compare shape, and statistical errors on the data only. 


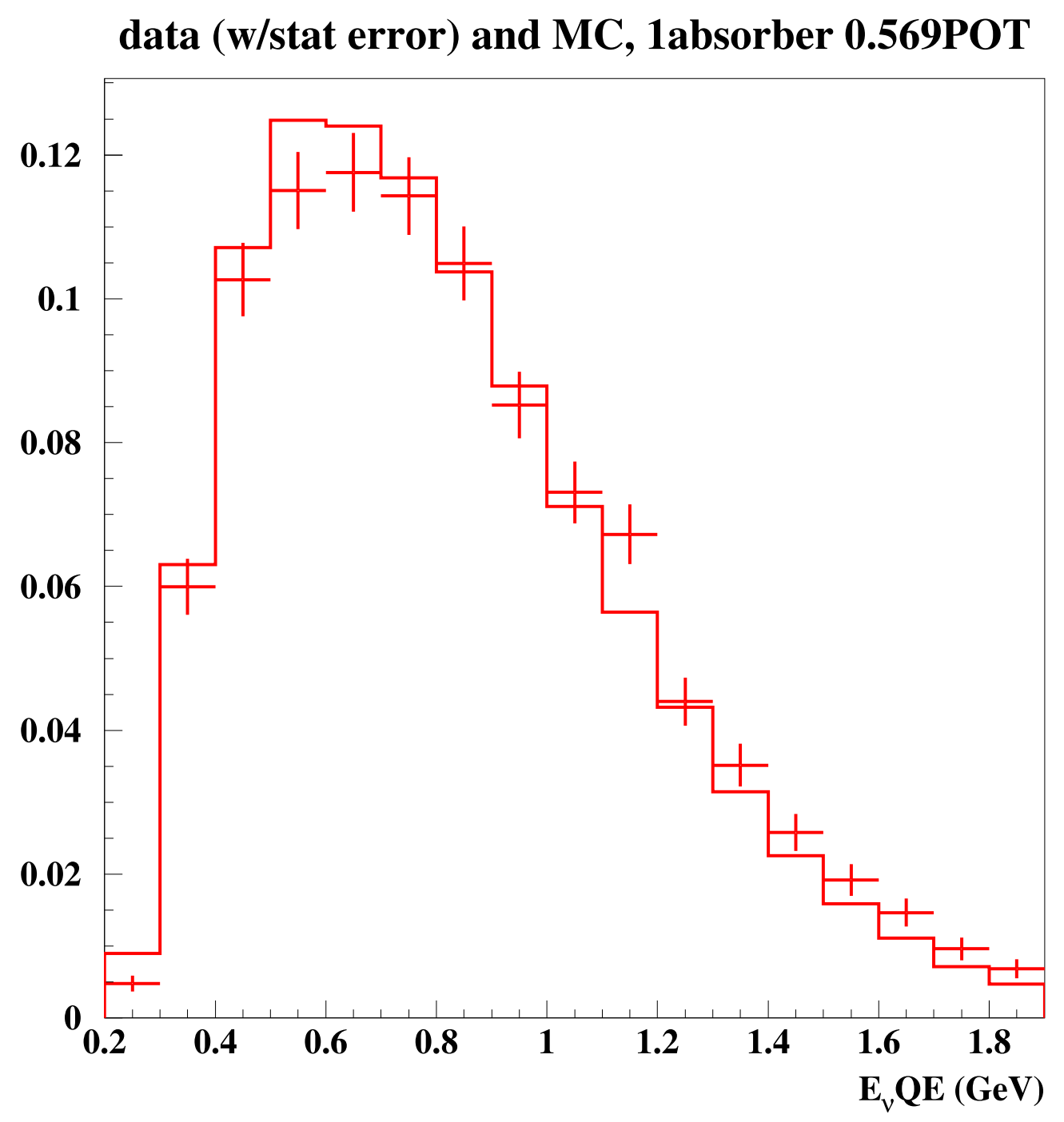

Figure 7.10: Data and unoscillated prediction vs. $E_{\nu}^{Q E}$ for the 1 absorber configuration, with unit normalization to better compare shape, and statistical errors on the data only. 


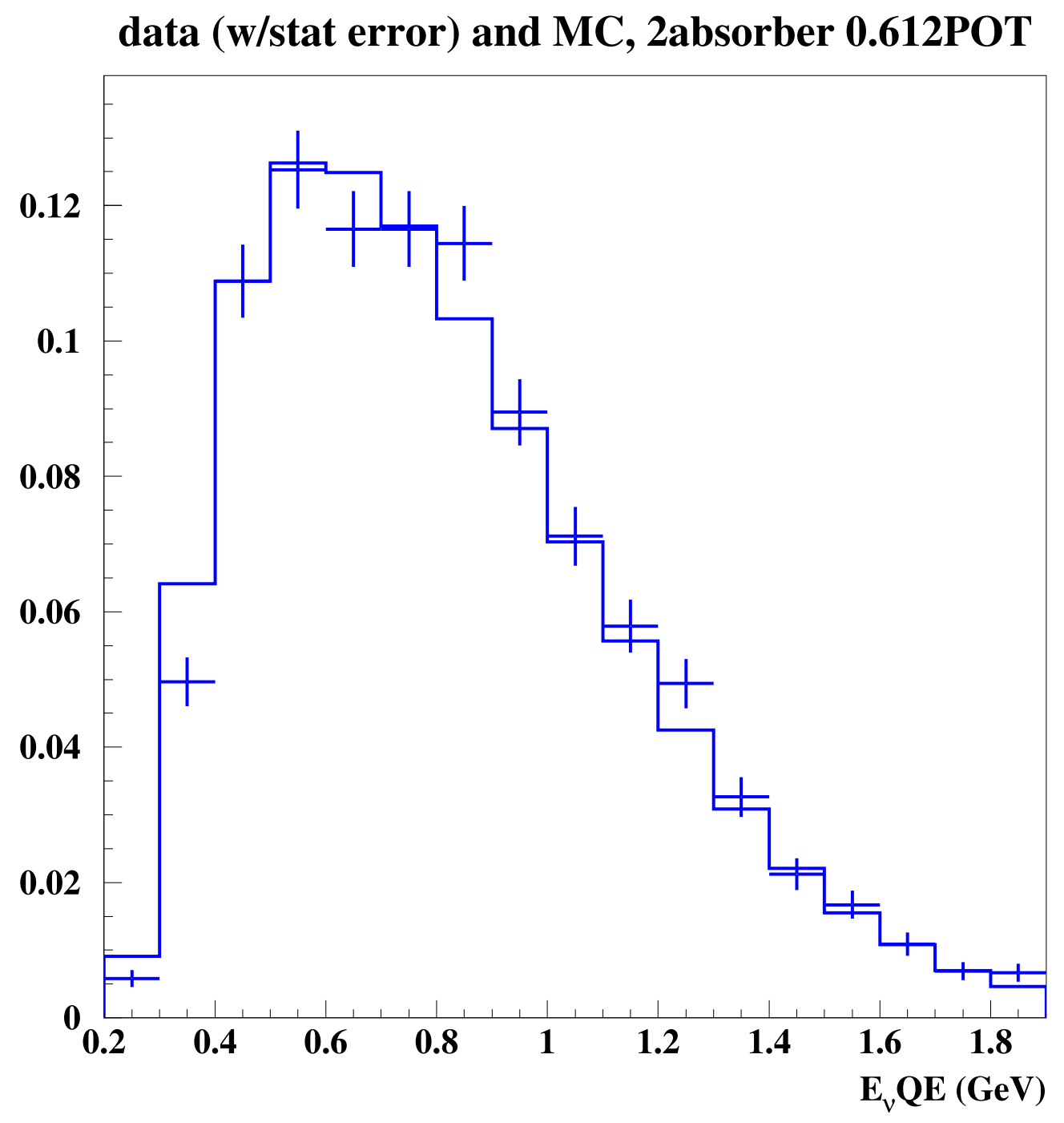

Figure 7.11: Data and $\mathrm{MC} E_{\nu}^{Q E}$ for the 2absorber configuration, with unit normalization to better compare shape, and statistical errors on the data only. 


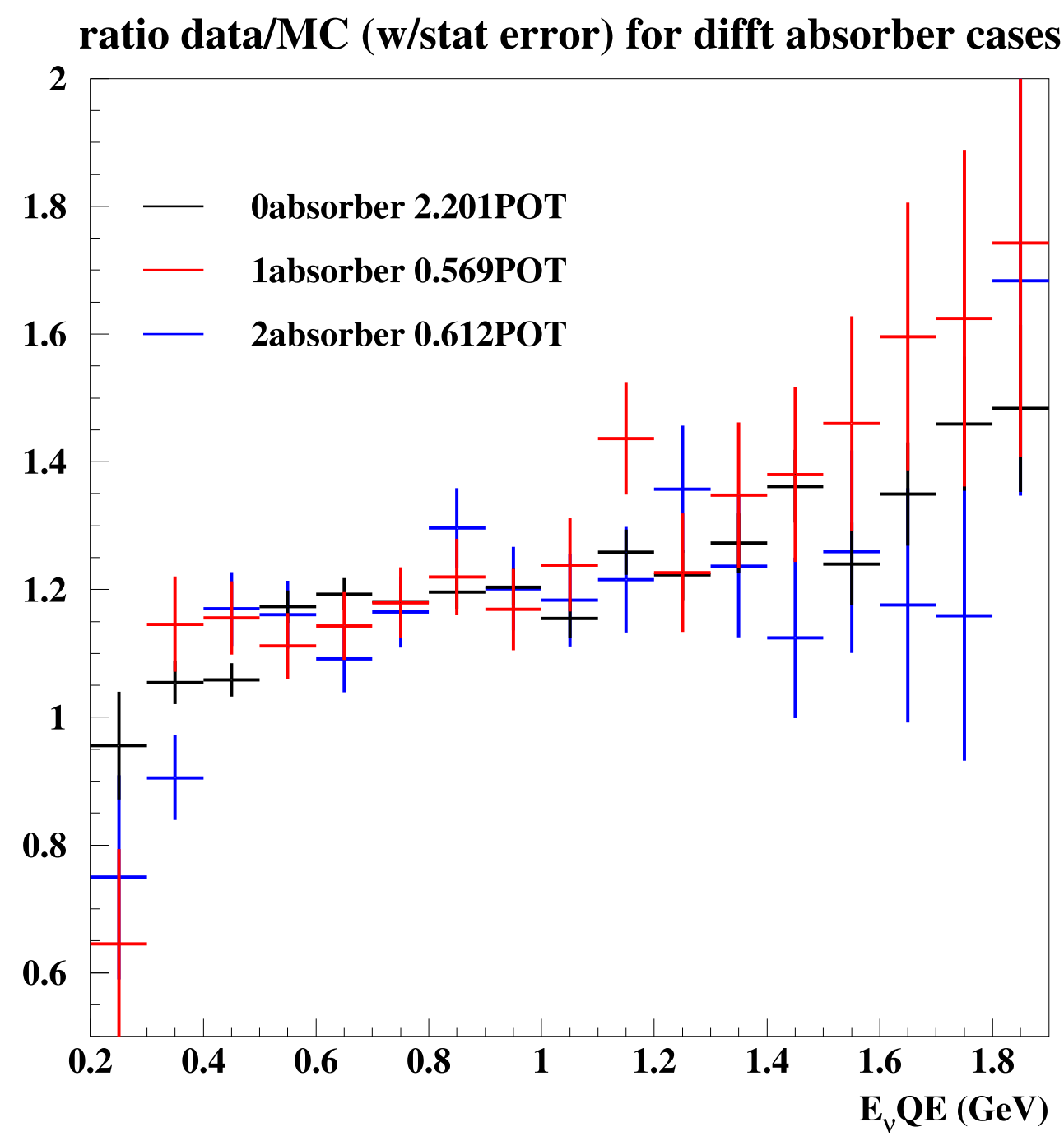

Figure 7.12: Ratio of data to prediction (POT normalized) $E_{\nu}^{Q E}$ for the three different absorber configurations, with statistical errors on the data only. $\mathrm{O}$ absorber is shown in black and corresponds to $2.201 \times 10^{20}$ POT. 1 absorber is shown in red and corresponds to $0.569 \times 10^{20}$ POT. 2 absorber is shown in blue and corresponds to $0.0612 \times 10^{20}$ POT. 


\begin{tabular}{|c|c|c|c|c|c|c|c|c|}
\hline bin boundaries & $0 .-0.4$ & $0.4-0.5$ & $0.5-0.6$ & $0.6-0.7$ & $0.7-0.8$ & $0.8-0.9$ & $0.9-1.0$ & $1.0-1.1$ \\
\hline prediction (total) & 1530.6 & 2274.8 & 2690.3 & 2742.6 & 2649.0 & 2413.5 & 2088.6 & 1719.3 \\
prediction (0 absorber) & 1058.0 & 1571.3 & 1873.6 & 1930.5 & 1888.6 & 1736.7 & 1516.5 & 1257.5 \\
data (0 absorber) & 1100.0 & 1660.0 & 2194.0 & 2299.0 & 2226.0 & 2073.0 & 1822.0 & 1449.0 \\
data (1 absorber) & 256.0 & 406.0 & 455.0 & 465.0 & 452.0 & 415.0 & 337.0 & 289.0 \\
data (2 absorher) & 209.0 & 410.0 & 472.0 & 439.0 & 439.0 & 431.0 & 337.0 & 268.0 \\
data total & 1565.0 & 2476.0 & 3121.0 & 3203.0 & 3117.0 & 2919.0 & 2496.0 & 2006.0 \\
\hline
\end{tabular}

Table 7.2: Total data $\left(3.386 \times 10^{20} \mathrm{POT}\right)$ and individual $0 \quad\left(2.205 \times 10^{20} \mathrm{POT}\right), \quad 1$ $\left(0.569 \times 10^{20} \mathrm{POT}\right)$ and 2 absorber $\left(0.612 \times 10^{20} \mathrm{POT}\right)$ configurations are shown with prediction, scaled to appropriate POT and including absorber configurations but unosccilated in energy bins from 0.-1.1 GeV.

\begin{tabular}{|c|c|c|c|c|c|c|c|c|}
\hline bin boundaries & $1.1-1.2$ & $1.2-1.3$ & $1.3-1.4$ & $1.4-1.5$ & $1.5-1.6$ & $1.6-1.7$ & $1.7-1.8$ & $1.8-1.9$ \\
\hline prediction (total) & 1384.6 & 1067.2 & 781.1 & 563.0 & 398.3 & 278.5 & 178.9 & 118.3 \\
prediction (0 absorber) & 1016.6 & 788.1 & 578.6 & 417.9 & 295.6 & 206.3 & 132.5 & 87.1 \\
data (0 absorber) & 1277.0 & 962.0 & 735.0 & 568.0 & 366.0 & 278.0 & 193.0 & 129.0 \\
data (1 absorber) & 266.0 & 174.0 & 139.0 & 102.0 & 76.0 & 58.0 & 38.0 & 27.0 \\
data (2 absorber) & 218.0 & 186.0 & 123.0 & 80.0 & 63.0 & 41.0 & 26.0 & 25.0 \\
data (total) & 1761.0 & 1322.0 & 997.0 & 750.0 & 505.0 & 377.0 & 257.0 & 181.0 \\
\hline
\end{tabular}

Table 7.3: Total data $\left(3.386 \times 10^{20}\right.$ POT $)$ and individual $0 \quad\left(2.205 \times 10^{20}\right.$ POT $), 1$ $\left(0.569 \times 10^{20} \mathrm{POT}\right)$ and 2 absorber $\left(0.612 \times 10^{20} \mathrm{POT}\right)$ configurations are shown with prediction, scaled to appropriate POT and including absorber configurations but unosccilated in energy bins from 1.1-1.9 GeV. 
error matrix. The prediction is relatively normalized to data by a factor of 1.18 . The $\chi^{2}$ of the null hypothesis is $13.7,8.2,15.2,10.29$ (16 DOF) for the zero, one, and two absorber plate and total data respectively, which is consistent with no oscillation at 90\%CL.

The limit to the full $\bar{\nu}_{\mu}$ dataset is shown in Fig. 7.14, where only $\bar{\nu}_{\mu}$ can oscillate. No $\bar{\nu}_{\mu}$ disappearance is observed at $90 \% \mathrm{CL}$, and this is the first measurement of $\bar{\nu}_{\mu}$ disappearance in the region of $\Delta m^{2}=0.1-10 \mathrm{eV}^{2}$, as only CCFR made a measurement of $\bar{\nu}_{\mu}$ disappearance in this region. No indication of CPT violation is observed with MiniBooNE data.

The minimum $\chi^{2}=5.43(11 \mathrm{DOF})$ is at $\Delta m^{2}=31.3 \mathrm{eV}^{2}, \sin ^{2}(2 \theta)=0.96$, where the number of degrees of freedom is estimated from frequentist $\Delta \chi^{2}$ studies. The $\chi^{2}$ at the minimum is "too good" with a probability of $90.8 \%$, but this is due to the inclusion of the 1 absorber data, which has a $\chi^{2}$ with no oscillation of $8.2(16 \mathrm{DOF}, 94.3 \%)$. The fit to the 0 absorber data only gives a reasonable $\chi^{2}$ at the minimum of $\chi^{2}=8.2(11 \mathrm{DOF}, 79.1 \%)$.

Provided as a cross check, the individual limit curves for the different absorber configuration data sets are also shown in Fig. 7.15, 7.16, 7.17 for zero, one, and two absorbers respectively. The fits to individual absorber data sets are consistent with the fit to the entire data set. 


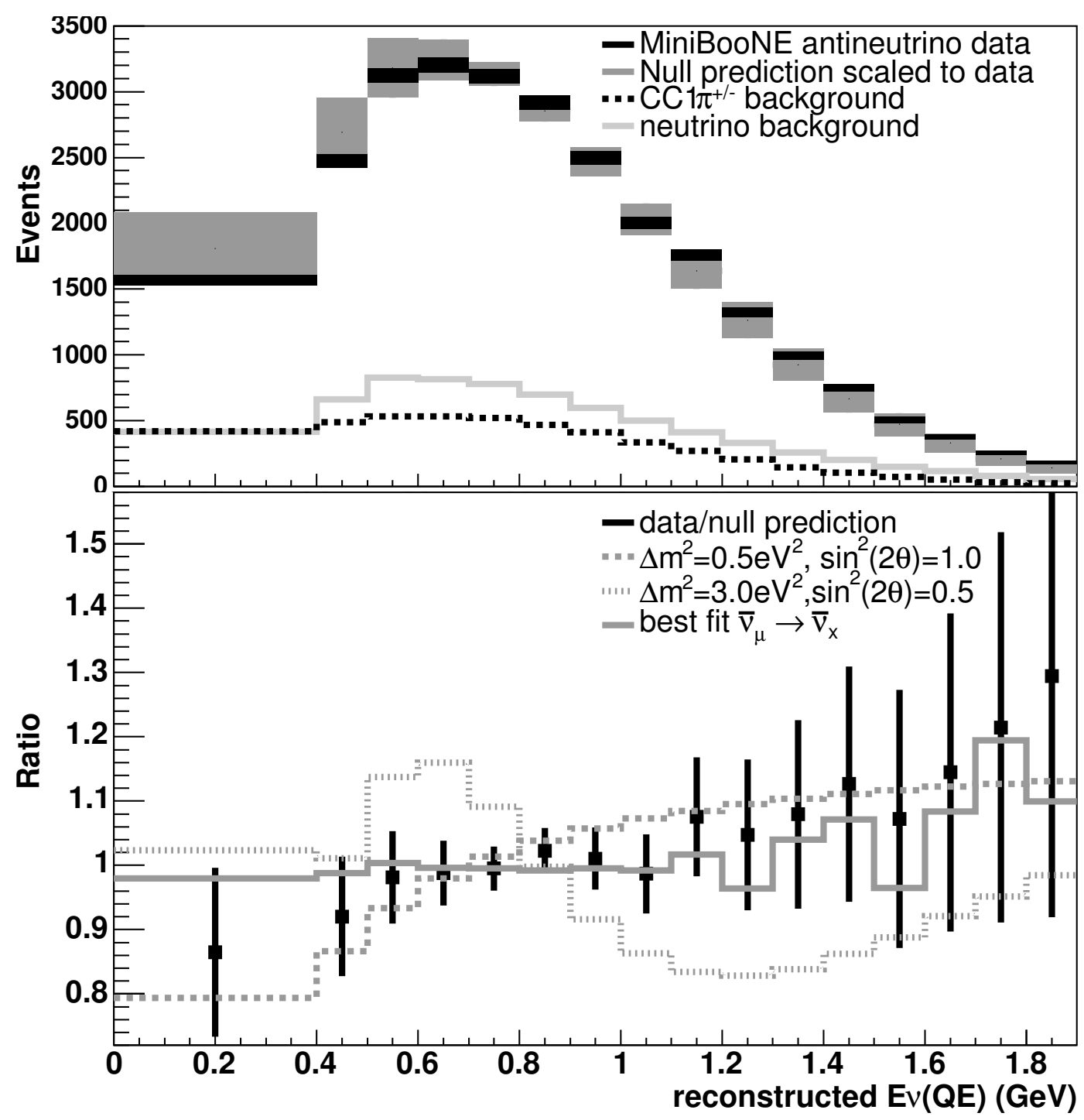

Figure 7.13: The top plot shows the $E_{\nu}^{Q E}$ distribution for antineutrino data (black) with statistical error rectangles (thickness of line indicates size of statistical error), and prediction assuming no oscillation (grey). Attached to the prediction are the diagonal elements of the shape error matrix. The predicted $\mathrm{CC} 1 \pi$ background (dash) and background neutrino (solid) events are also shown. The bottom plot shows the ratio of data to no oscillation (black), and the ratio of no oscillation to: $\Delta m^{2}=0.5 \mathrm{eV}^{2}, \sin ^{2}(2 \theta)=1.0$ disappearance (dashed line), $\Delta m^{2}=3.0 \mathrm{eV}^{2}, \sin ^{2}(2 \theta)=0.5$ disappearance (dotted line) and for the minimum $\chi^{2}=5.43(11 \mathrm{DOF})$ is at $\Delta m^{2}=31.3 \mathrm{eV}^{2}, \sin ^{2}(2 \theta)=0.96$. Only antineutrino events are oscillated. 


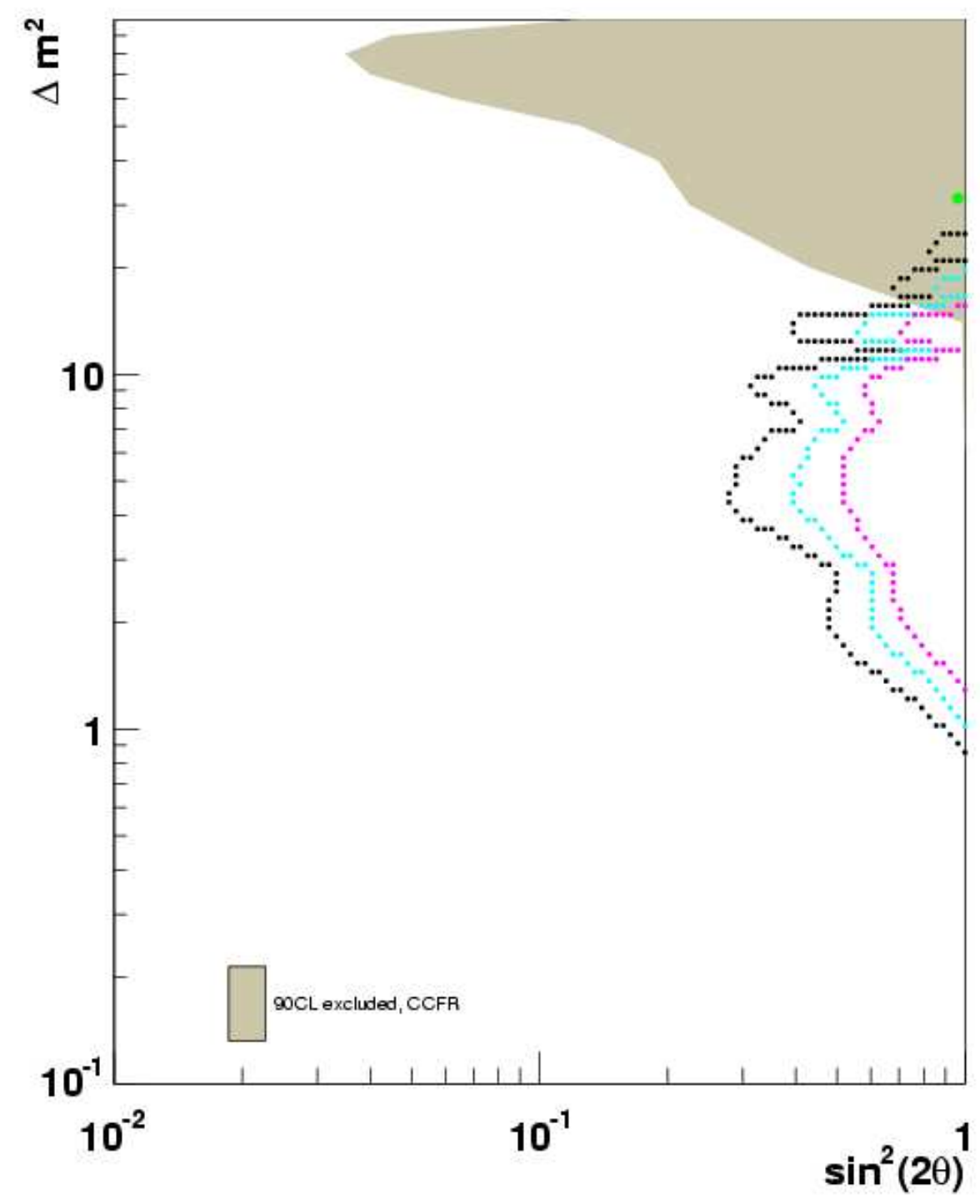

Figure 7.14: Shape only Pearson $\chi^{2}$ method 90\% CL (black) $3 \sigma$ (cyan) and $5 \sigma$ (magenta) limit curves for the CCQE $\bar{\nu}_{\mu}$ disappearance analysis fit to data $\left(3.386 \times 10^{20}\right)$ where only antineutrinos are allowed to oscillate (neutrinos remain fixed). The 90\% CL exclusion regions for CCFR (light brown) is also shown. $\chi^{2}$ (null)=10.29 (16 DOF probability: 85.1\%) and $\chi^{2}$ (minimum) $=5.42(11 \mathrm{DOF}$, probability: $96 \%)$ at $\Delta m^{2}=31.32 \mathrm{eV}^{2}, \sin ^{2}(2 \theta)=0.96$. 


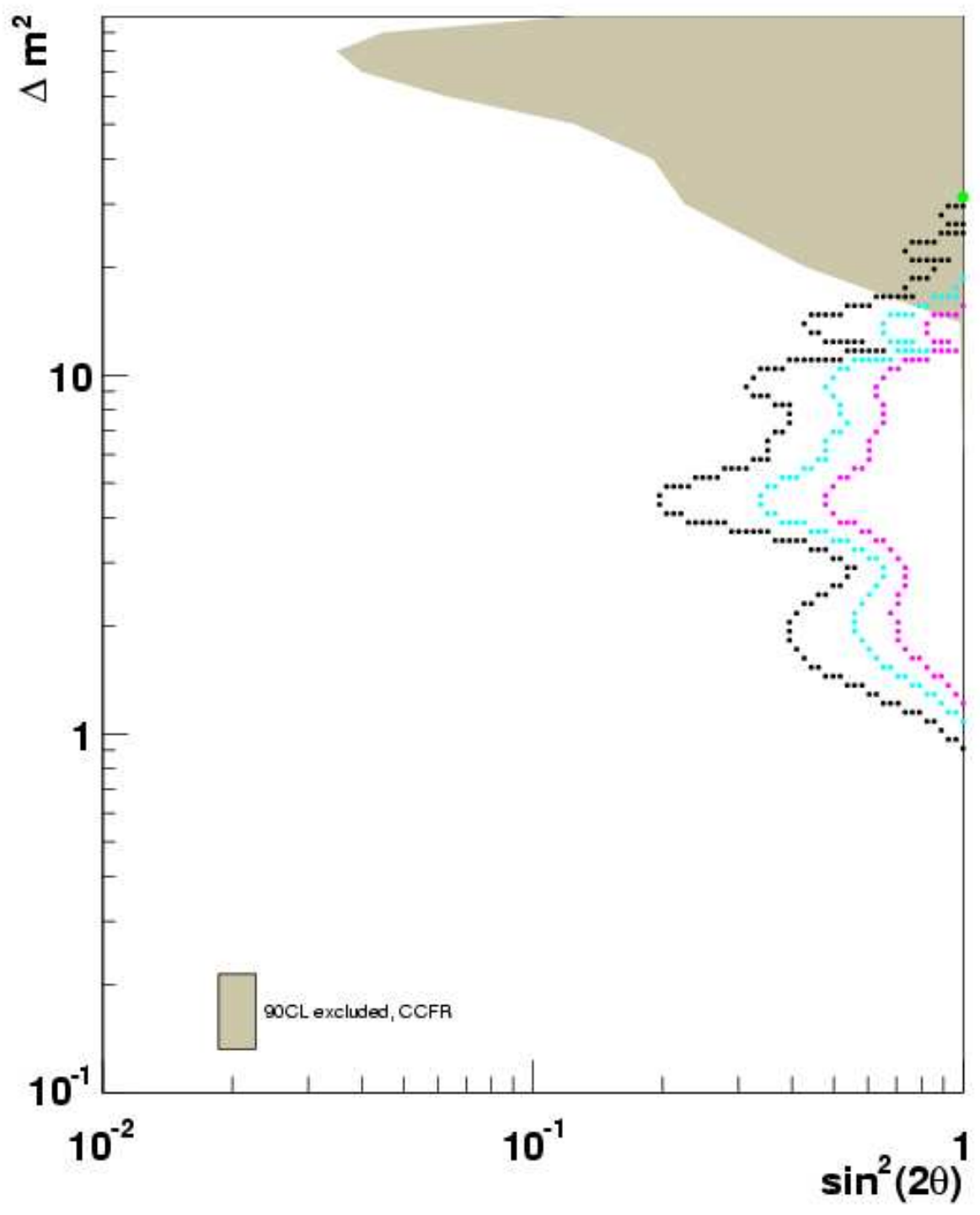

Figure 7.15: Shape only Pearson $\chi^{2}$ method 90\% CL (black) $3 \sigma$ (cyan) and $5 \sigma$ (magenta) limit curves for the CCQE $\bar{\nu}_{\mu}$ disappearance analysis fit to 0 absorber data $\left(2.2 \times 10^{20}\right)$ where only antineutrinos are allowed to oscillate (neutrinos remain fixed). The $90 \%$ CL exclusion regions for CCFR (light brown) is also shown. $\chi^{2}$ (null)=13.74 (16 DOF probability: $61.8 \%$ ) and $\chi^{2}$ (minimum) $=7.07$ at $\Delta m^{2}=31.32 \mathrm{eV}^{2}, \sin ^{2}(2 \theta)=1.0$ 


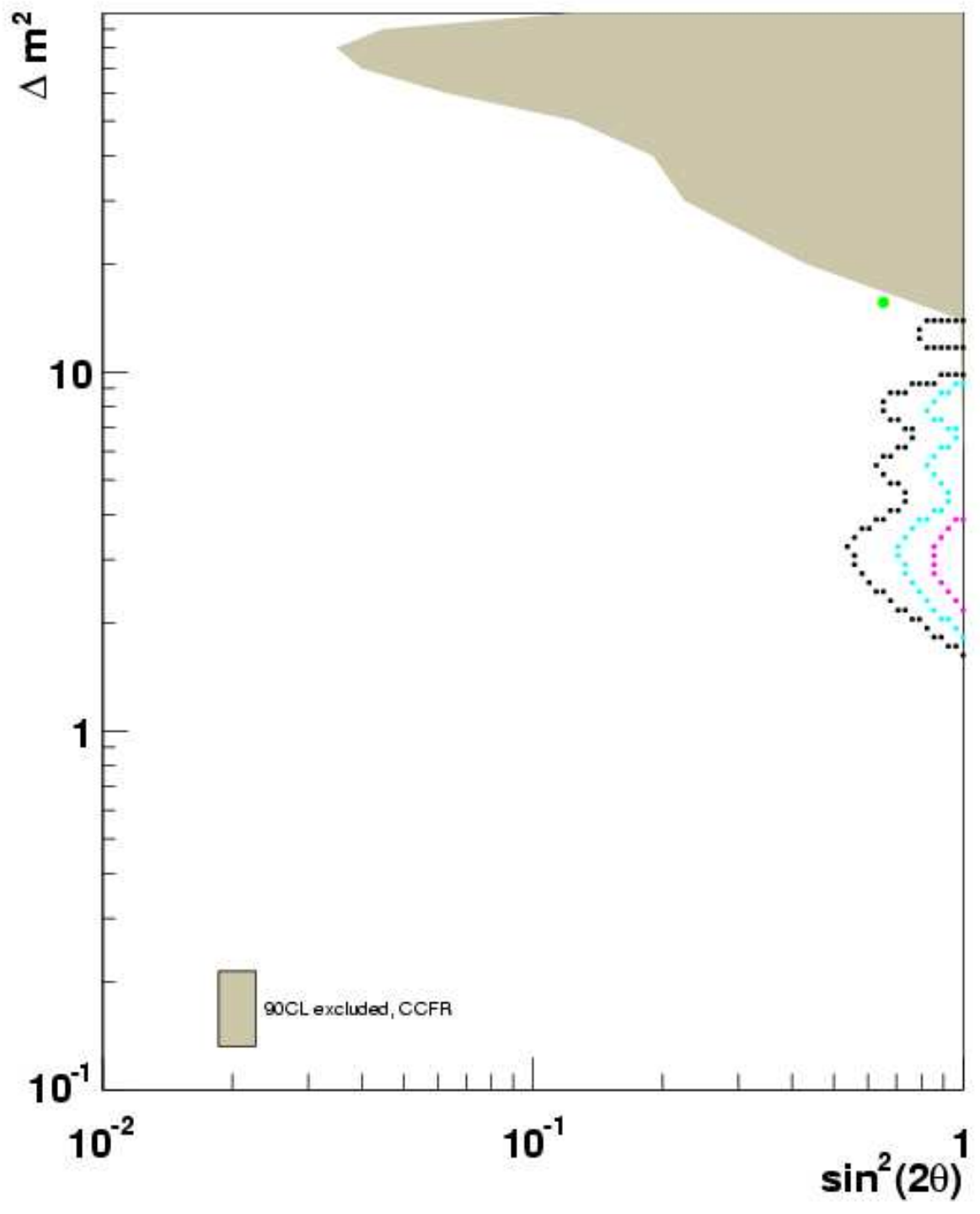

Figure 7.16: Shape only Pearson $\chi^{2}$ method 90\% CL (black) $3 \sigma$ (cyan) and $5 \sigma$ (magenta) limit curves for the CCQE $\bar{\nu}_{\mu}$ disappearance analysis fit to 1 absorber data $\left(0.5 \times 10^{20}\right)$ where only antineutrinos are allowed to oscillate (neutrinos remain fixed). The $90 \%$ CL exclusion regions for CCFR (light brown) is also shown. $\chi^{2}$ (null)=8.17 (16 DOF probability: $94.4 \%$ ) and $\chi^{2}$ (minimum) $=4.64$ at $\Delta m^{2}=15.6 \mathrm{eV}^{2}, \sin ^{2}(2 \theta)=0.65$. 


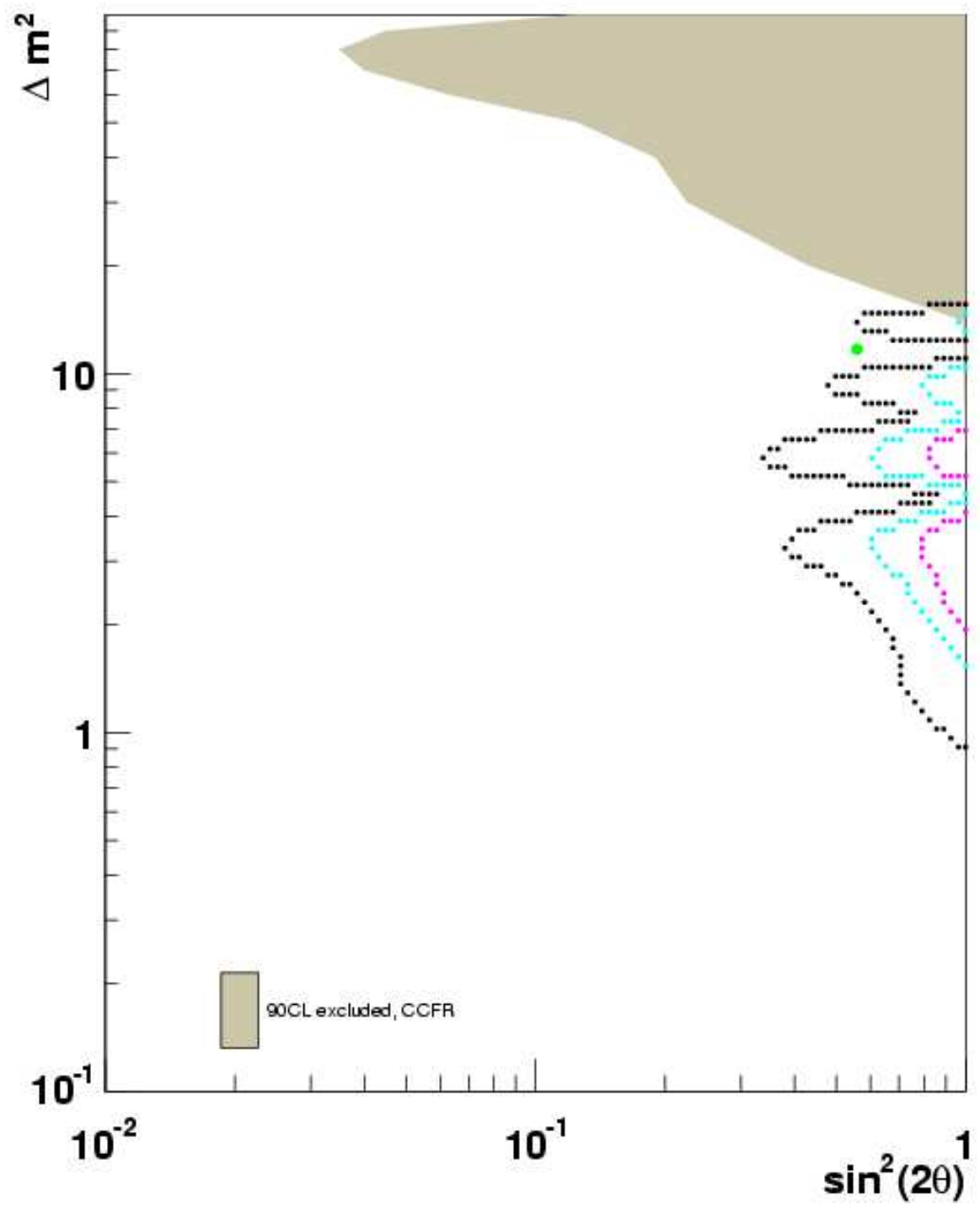

Figure 7.17: Shape only Pearson $\chi^{2}$ method 90\% CL (black) $3 \sigma$ (cyan) and $5 \sigma$ (magenta) limit curves for the CCQE $\bar{\nu}_{\mu}$ disappearance analysis fit to 2 absorber data $\left(0.6 \times 10^{20}\right)$ where only antineutrinos are allowed to oscillate (neutrinos remain fixed). The $90 \%$ CL exclusion regions for CCFR (light brown) is also shown. $\chi^{2}$ (null)=15.21 (probability: $50.9 \%$ ) and $\chi^{2}($ minimum $)=12.03$ at $\Delta m^{2}=11.67 \mathrm{eV}^{2}, \sin ^{2}(2 \theta)=0.56$. 


\section{Chapter 8}

\section{$\nu_{\mu}$ disappearance with MiniBooNE and SciBooNE}

Section 6 described a single detector disappearance experiment using MiniBooNE. The MiniBooNE measurement was a shape only measurement, because of the substantial normalization $(\sim 20 \%)$ uncertainties. The two largest errors on the shape measurement, as shown in Figure 6.6, are the flux and the cross section. To make an improved measurement of disappearance with MiniBooNE, these two sources of uncertainty must be better understood.

Historically, disappearance experiments use two detectors, one at the 'far' site, where oscillations are occurring, and one at a 'near' site, where neutrinos have not oscillated yet. The reason to use a near detector is to constrain the unoscillated rate (flux $\times$ cross section). Ideally, one would want to use two identical detectors at near and far sites, more commonly a detector is placed so it observes a similar flux and is constructed to be of the same material (neutrino target) as the far detector. A third type of constraint is to constrain just the cross section using previous measurements on the appropriate neutrino target.

MiniBooNE was designed explicitly to search for short baseline neutrino oscillations at the LSND $\Delta m^{2}$, but SciBooNE is predominantly cross section experiment. The T2K experiment is a long baseline experiment with a peak energy just under $1 \mathrm{GeV}$. Though 


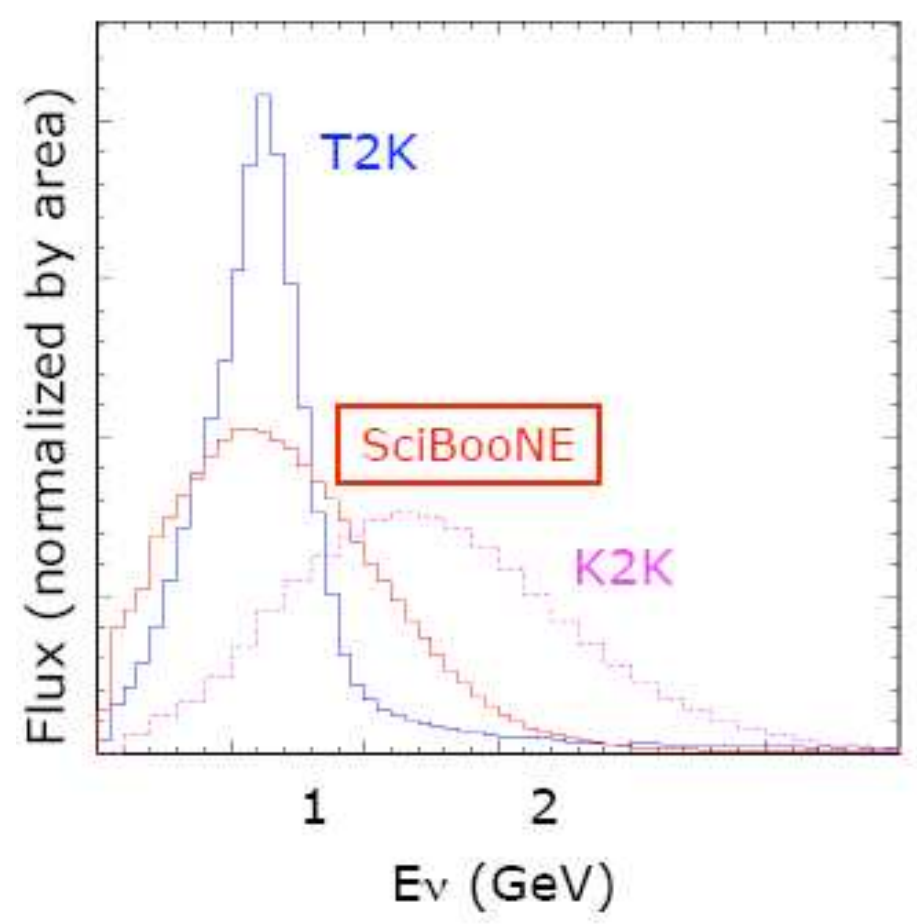

Figure 8.1: The neutrino energy spectrum, normalized by area for three experiments, the T2K experiment (blue), the BNB (SciBooNE/MiniBooNE experiments) (red) and the K2K experiment (pink, dashed line).

T2K will employ a series of near detectors to constrain the rate at the far detector, the uncertainties in the cross section in this neutrino energy range are substantial for neutrinos (Fig. 4.16) and no experiments exist with antineutrinos (Fig. 4.17). A dedicated cross section experiment, SciBooNE, was proposed to make measurements of the processes important in the $\sim 1 \mathrm{GeV}$ region. The neutrino energy spectrum for $\mathrm{T} 2 \mathrm{~K}$ is shown with $\mathrm{K} 2 \mathrm{~K}$ and the BNB in Fig. 8.1. K2K's neutrino beam is too high to help constrain the narrower T2K beam, but the BNB covers the correct region and has the capability to make both neutrino and antineutrino measurements. The location of SciBooNE in the neutrino beamline was based on an optimization between rate and neutrino energy spectrum; it was found to be best to remain directly in the neutrino beamline and close to the neutrino source to get as high a rate as possible.

While SciBooNE was proposed to make cross section measurements, the SciBar detector is the same neutrino target as MiniBooNE, carbon. Therefore, SciBooNE can poten- 
tially become a near detector for MiniBooNE, constraining the flux and cross section and improving the ability to search for $\nu_{\mu}$ and $\bar{\nu}_{\mu}$ disappearance.

This chapter discusses the prospects for a joint SciBooNE-MiniBooNE disappearance analysis. First, we discuss the the power of constraints: how an unoscillated detector reduces the effective error on the far detector. Then we consider an oscillating near detector. We show a preliminary sensitivity combining the a SciBooNE sample with the MiniBooNE sample for neutrino mode. Finally, we discuss improvements to this joint analysis and prospects for antineutrino mode.

\subsection{How Constraints Work}

A near detector provides a measurement of the unoscillated rate (flux $\times$ cross section $\times$ efficiency), which constrains the far detector rate. A simple example with identical detectors illustrates the power of constraints.

\subsubsection{Simple Two-Bin Cases}

There are two ways to use the near detector measurement:

- Case 1: Apply the measurement to the far detector rate, and reduce the far detector rate uncertainties accordingly

- Case 2: Fit both near detector and far detector simultaneously.

In case 1 , the near detector measures the rate $N \pm \sigma_{n}+\delta_{n}$, where $\delta_{n}$ is the systematic uncertainty and $\sigma_{n}=N$ is the statistical uncertainty. The far detector rate $F$, is corrected using $N$ using a prediction relating the far and near detectors ("far to near ratio") $F=\frac{f}{n} N$.

The uncertainty on the corrected prediction $F$ will depend on the uncertainty on the far to near ratio:

$$
\begin{aligned}
& \frac{\delta_{F}^{2}}{F^{2}}=\frac{\delta_{f / n}^{2}}{f / n^{2}}+\frac{\sigma_{n}^{2}}{n^{2}}+\frac{\sigma_{f}^{2}}{f^{2}} \\
& \frac{\delta_{f / n}^{2}}{f / n^{2}}=\frac{\delta_{n}^{2}}{n^{2}}+\frac{\delta_{f}^{2}}{f^{2}}-2 \frac{\rho \delta_{n} \delta_{f}}{n f}
\end{aligned}
$$

If changes to the far detector rate $f$ do not change $n$, then $\rho=0$, and the error on $F$ includes all uncertainties on $n$ and $f$, and there would be no reason to add the near 
detector measurement. One instead would just measure the far detector's disappearance $\Delta=F-f \pm \sigma_{f}+\delta_{f}$. If the changes to the far detector rate $f$ are fully correlated with $n$, then $\rho=1$, and the relative change is the same in both detectors $\left(\frac{\delta_{f}}{f}=\frac{\delta_{n}}{n}\right)$. Then the uncertainty on $F$ is minimized and becomes the size of the statistical error on the near and far detectors added in quadrature, $\delta_{F}=F \sqrt{\frac{\sigma_{n}^{2}}{n^{2}}+\frac{\sigma_{f}^{2}}{f^{2}}}$.

In the second case, consider a simultaneous fit to $N$ and $F$ for disappearance. The $\chi^{2}$ would compare the prediction $p_{1}=n, p_{2}=f$ to the observation $d_{1}=N, d_{2}=F$ with an error matrix $M$ :

$$
\begin{aligned}
\chi^{2} & =\sum_{i, j}^{n, f}\left(d_{i}-p_{i}\right) M_{i j}{ }^{-1}\left(d_{j}-p_{j}\right) \\
M & =\left(\begin{array}{cc}
\sigma_{n}^{2}+\delta_{n}^{2} & \rho \delta_{n} \delta_{f} \\
\rho \delta_{f} \delta_{n} & \sigma_{f}^{2}+\delta_{f}^{2}
\end{array}\right)
\end{aligned}
$$

The value of disappearance observable $\Delta$ occurs when this $\chi^{2}$ is minimized at:

$$
\Delta=(F-f)\left[1-\frac{\rho}{\frac{\sigma_{n}}{\delta_{n}}+1} \frac{\frac{N-n}{\delta_{n}}}{\frac{F-f}{\delta_{f}}}\right]
$$

The uncertainty on a disappearance measurement from minimizing this $\chi^{2}$ is:

$$
\delta_{\Delta}^{2}=\sigma_{f}^{2}+\delta_{f}^{2}\left(1-\frac{\rho^{2}}{\left(\sigma_{n} / \delta_{n}+1\right)}\right)
$$

When $\rho=0$, the near detector does nothing, $\Delta=(F-f) \pm \sigma_{f}^{2}+\delta_{f}^{2}$, and one makes a far detector only measurement. When the far and near detectors are perfectly correlated, then the systematic uncertainty on the far detector is scaled by the ratio of the systematic errors to the statistical errors in the near detector. When the statistical errors are equal to the systematic uncertainties: $\delta_{\Delta}^{2}=\sigma_{f}^{2}+\frac{1}{2} \delta_{f}^{2}$, and when the statistical uncertainties on the near detector are small, then $\delta_{\Delta}^{2}=\sigma_{f}^{2}$ and the systematic uncertainty is removed entirely.

In both methods, we trade the systematic uncertainty on the far detector for the statistical (or uncorrelated) error on the near detector for an improved measurement on the far detector. Case 1 and Case 2 are both being employed for the joint MiniBooNE/SciBooNE analysis, but Case 2 is detailed here. 


\subsection{Including A Near Detector in Disappearance Fits}

To include a near detector in the disappearance analysis is identical to the procedure used to fit MiniBooNE. The $\chi^{2}$ is formed over 32 reconstructed energy bins, including 16 additional bins for the near detector:

$$
\chi^{2}=\sum_{i, j}^{32 b i n s}\left(d_{i}-p_{i}\right) M_{i j}^{-1}\left(d_{j}-p_{j}\right)
$$

Now the error matrix, $M_{i j}$, is a $32 \times 32$ matrix ranging over reconstructed energy bins in the near detector (SciBooNE) and the far detector (MiniBooNE). This fit can be shapeonly or include both shape and normalization information. The $90 \%$ CL value for a $\chi^{2}$ function corresponding to $32 \mathrm{DOF}$ is 42.5 , and is used for all near+far detector fits shown in this chapter.

\subsubsection{Systematic Uncertainties in SciBooNE and MiniBooNE}

The error matrix is again the sum of the flux, cross section and detector error matrices. The flux matrix and cross section error matrices is formed in the same manner as discussed in Section 6.3.1 and Section 6.3.2, but now the prediction, $p_{i}$, is formed using SciBooNE and MiniBooNE events. Each draw is the same for both experiments (e.g. $M_{A}=1.0 \rightarrow$ 1.23), and shared parameters between the two predictions are the same (flux prediction and cross section prediction). The MiniBooNE detector uncertainties are kept the same as in the MiniBooNE-only analysis, but are a 16x16 matrix uncorrelated to SciBooNE. The dominant detector uncertainty on the SciBooNE CC sample is due to the uncertainty in the thickness of the iron plates. Preliminary detector uncertainties used a $2 \%$ uncorrelated error for the SciBooNE.

A summary of the normalization uncertainties on SciBooNE and MiniBooNE is given in Table 8.1. The flux uncertainty is larger for the SciBooNE CC inclusive sample because of larger uncertainties due to the horn magnetic field and a larger background of $\nu_{\mu}$ from $K^{+}$. The SciBooNE cross section uncertainty is lower than MiniBooNE because less events are CCQE; the MiniBooNE cross section uncertainty is lower than for the MiniBooNE-only analysis because the CCQE cross section model dependance was not included. 


\begin{tabular}{|c|l|c|}
\hline uncertainty type & experiment & normalization uncertainty \\
\hline \hline flux & MiniBooNE & $5.8 \%$ \\
\hline & SciBooNE & $8.1 \%$ \\
\hline \hline cross section & MiniBooNE & $14.3 \%$ \\
\hline & SciBooNE & $12.9 \%$ \\
\hline \hline detector & MiniBooNE & $3.4 \%$ \\
\hline & SciBooNE & $2.0 \%$ \\
\hline \hline
\end{tabular}

Table 8.1: Summary of systematic uncertainties considered in the joint SciBooNE/MiniBooNE disappearance fit. The third column indicates the normalization uncertainty due to each source of systematic error (Eq. 6.9).

The (fractional) size of the diagonal elements of the shape-only error matrix (total, flux, cross section and detector) is shown in Fig. 8.2 for the SciBooNE events. The correlations between SciBooNE and MiniBooNE are shown in in Fig. 8.3; the upper right quadrant is the correlation for MiniBooNE bins, which (correctly) looks like Fig. 6.3. The correlations are strong between MiniBooNE and SciBooNE samples, which will be exploited in the joint fit.

The next three sections examine different scenarios, starting with an ideal near detector and ending with the SciBooNE sample discussed in Section 5.1.3.

- A perfect near detector, unoscillated

- A perfect near detector, oscillated

- A realistic near detector (SciBooNE CC inclusive sample), oscillated

\subsubsection{Perfect Near Detector, Unoscillated}

A perfect near detector to MiniBooNE would have the same neutrino energy spectrum with the same flux and cross section. The power of the constraint is demonstrated in Fig. 8.4. A single, "far" detector 90\%CL sensitivity to $\nu_{\mu}$ disappearance is shown for a 16 bin Pearson's $\chi^{2}$ shape+normalization fit using "flux" and detector errors. The flux 


\section{Diagonal elements of shape matrix}

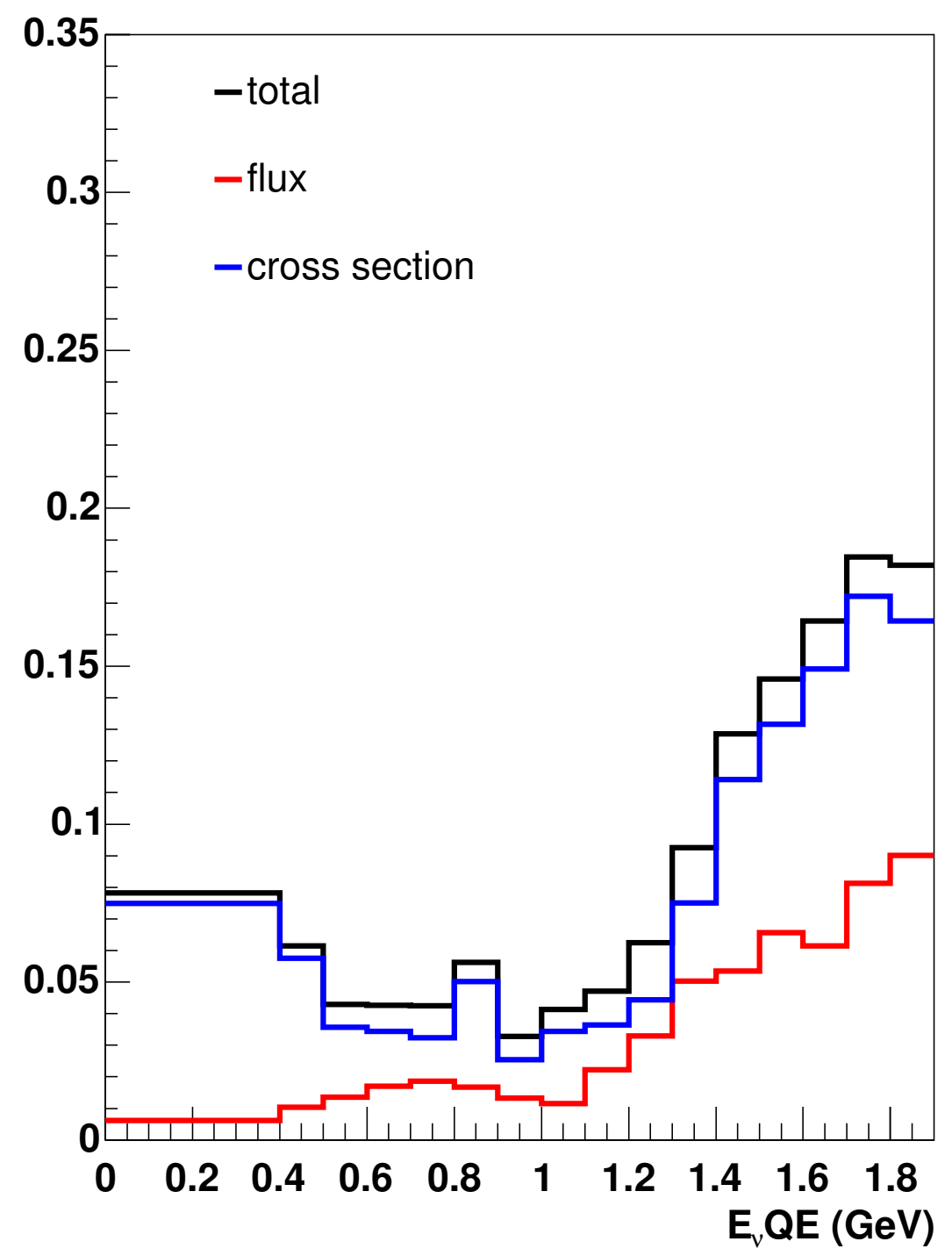

Figure 8.2: Diagonal elements of the shape-only error matrix $\left(\sqrt{M_{i i}^{\text {shape }}} / p_{i i}\right)$ vs. $E_{\nu}^{Q E}$ for the SciBooNE CC inclusive sample. The shape-only error matrix is calculated using Eq. 6.10. The total shape-only error matrix (black) is the sum of the flux (red), cross section (blue) and detector (2\% uncertainty) error matrices. 


\section{Correlations of the shape-only error matrix}

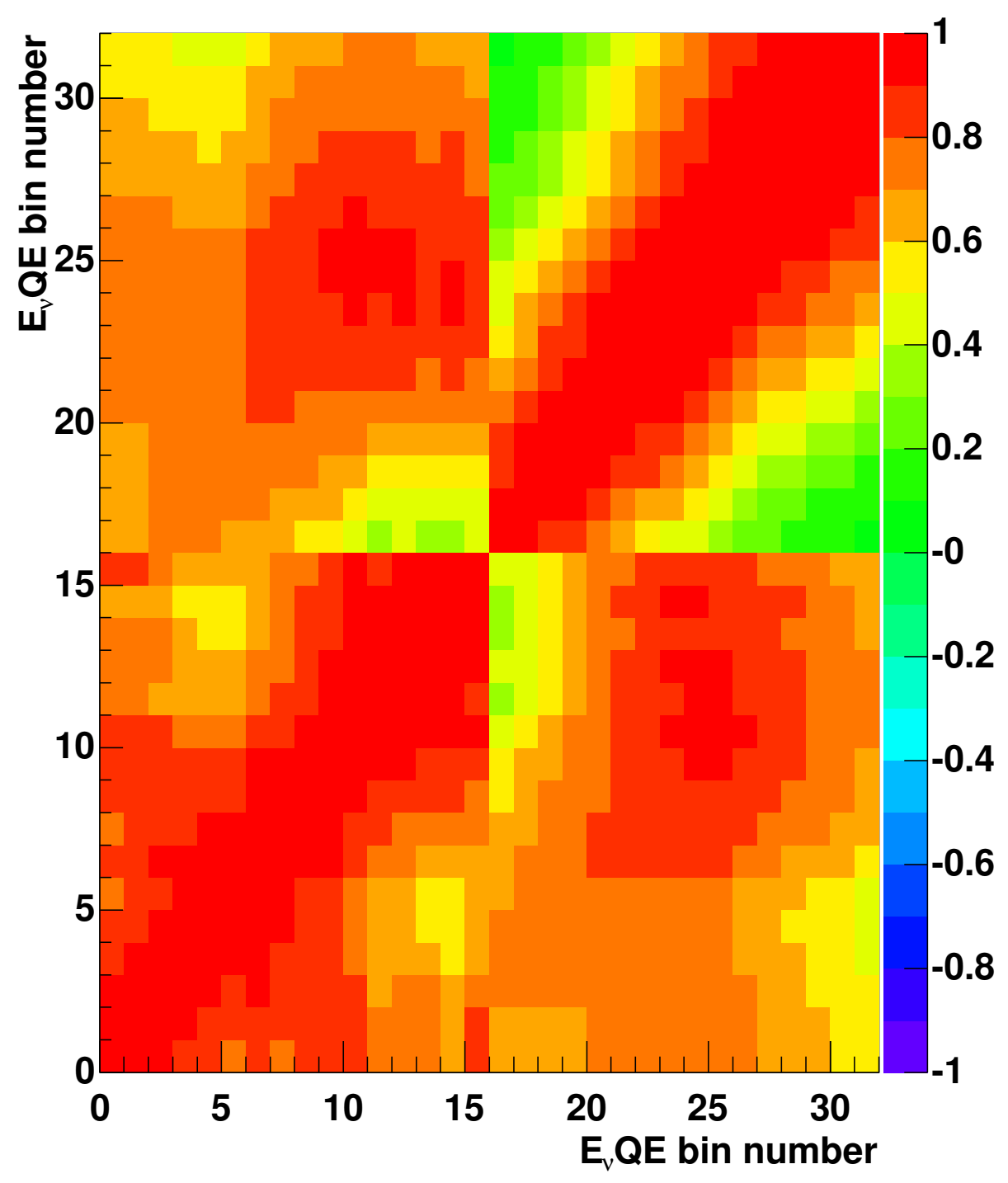

Figure 8.3: The correlation $\rho_{i j}=M_{i j}^{\text {shape }} / \sqrt{\left(M_{i i}^{\text {shape }} M_{j j}^{\text {shape }}\right)}$ of the total error matrix vs. $E_{\nu}^{Q E}$ bins. The shape-only error matrix is calculated using Eq. 6.10. Bins 0-16 are SciBooNE, bins 17-32 are MiniBooNE. 
errors are constructed to be a predominantly normalization uncertainty with some small shape component. The far detector has the same energy spectrum and detector uncertainties as MiniBooNE. A second, unoscillated "near"detector is included, with the identical spectrum as MiniBooNE, and a 32 bin Pearson's $\chi^{2}$ fit is performed. The improvement in the sensitivity is dramatic, by design. At high $\Delta m^{2}$ which corresponds to a normalization change in the far detector, the joint fit to the near detector removes all systematic uncertainty corresponding to the flux to the level of the near detector's statistical error; the fit gives almost the same sensitivity if the flux error was not included at all. At $\Delta m^{2} \sim 1 \mathrm{eV}^{2}$, the near detector constrains the shape of the flux. The difference between the joint fit and the far detector only fit at $\Delta m^{2} \sim 6 \mathrm{eV}^{2}$ arise from 16 bin and 32 bin method; the 32 bin method produces a slightly worse sensitivity than the 16 bin method for regions where there is no constraint from the near detector.

\subsubsection{Perfect Near Detector, Oscillated}

Now, include oscillations in the perfect near detector at 100 n (like SciBooNE). Oscillations at the perfect near detector and the far (MiniBooNE) detector are shown in Fig. 8.5. Below $\Delta m^{2} \sim 1 \mathrm{eV}^{2}$, the near detector at $100 \mathrm{~m}$ does not oscillate, but for larger $\Delta m^{2}$, the near detector also oscillates.

The effect of an oscillating near detector the $90 \%$ sensitivity is shown in Figure 8.6. Below $\Delta m^{2} \sim 1 \mathrm{eV}^{2}$, where the near detector does not oscillate, the constraint is the same as seen in Fig. 8.4. Above $\Delta m^{2} \sim 1 \mathrm{eV}^{2}$, where the near detector oscillates, the sensitivity is comperable to a measurement made with just the near detector. The 16 bin disappearance fit is slightly better than the 32 bin version as mentioned above.

\subsubsection{A Realistic Near Detector (SciBooNE)}

Figure 8.7 shows oscillations in the SciBooNE sample compared to the MiniBooNE sample as a function of reconstructed neutrino energy. The higher relative $\mathrm{CC} \pi^{+}$content in the SciBooNE CC inclusive sample reduces the size of oscillations relative to the perfect near detector. 


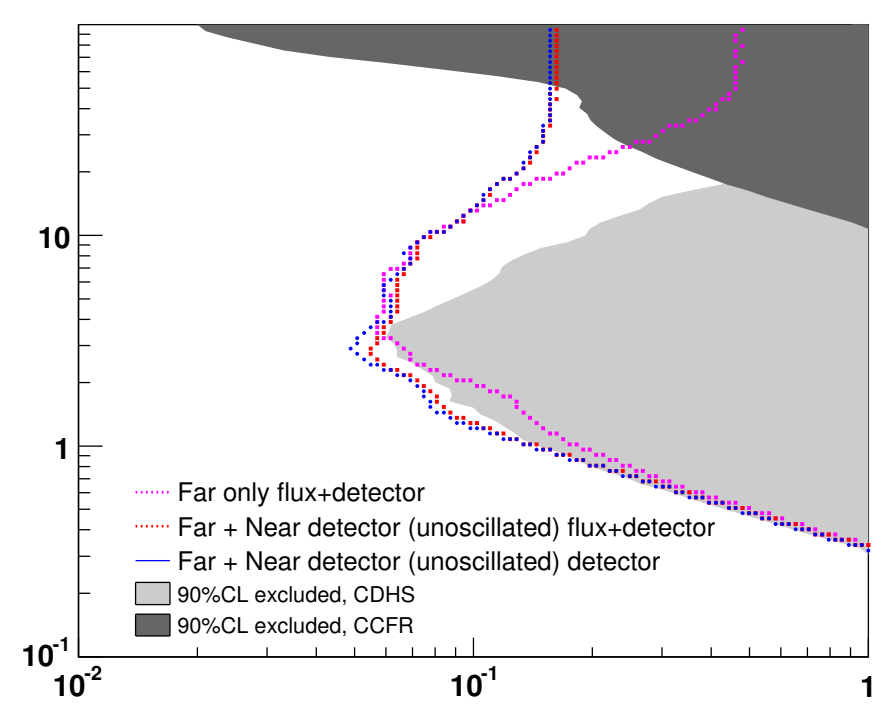

Figure 8.4: The 90\% CL sensitivity for: a single 16 bin far detector fit using flux and detector uncertainties (pink), a joint near+far detector 32 bin fit using flux and detector uncertainties (red), and a joint near+far detector 32 bin fit using detector uncertainties. The near detector does not oscillate. All fits include shape and normalization information. 

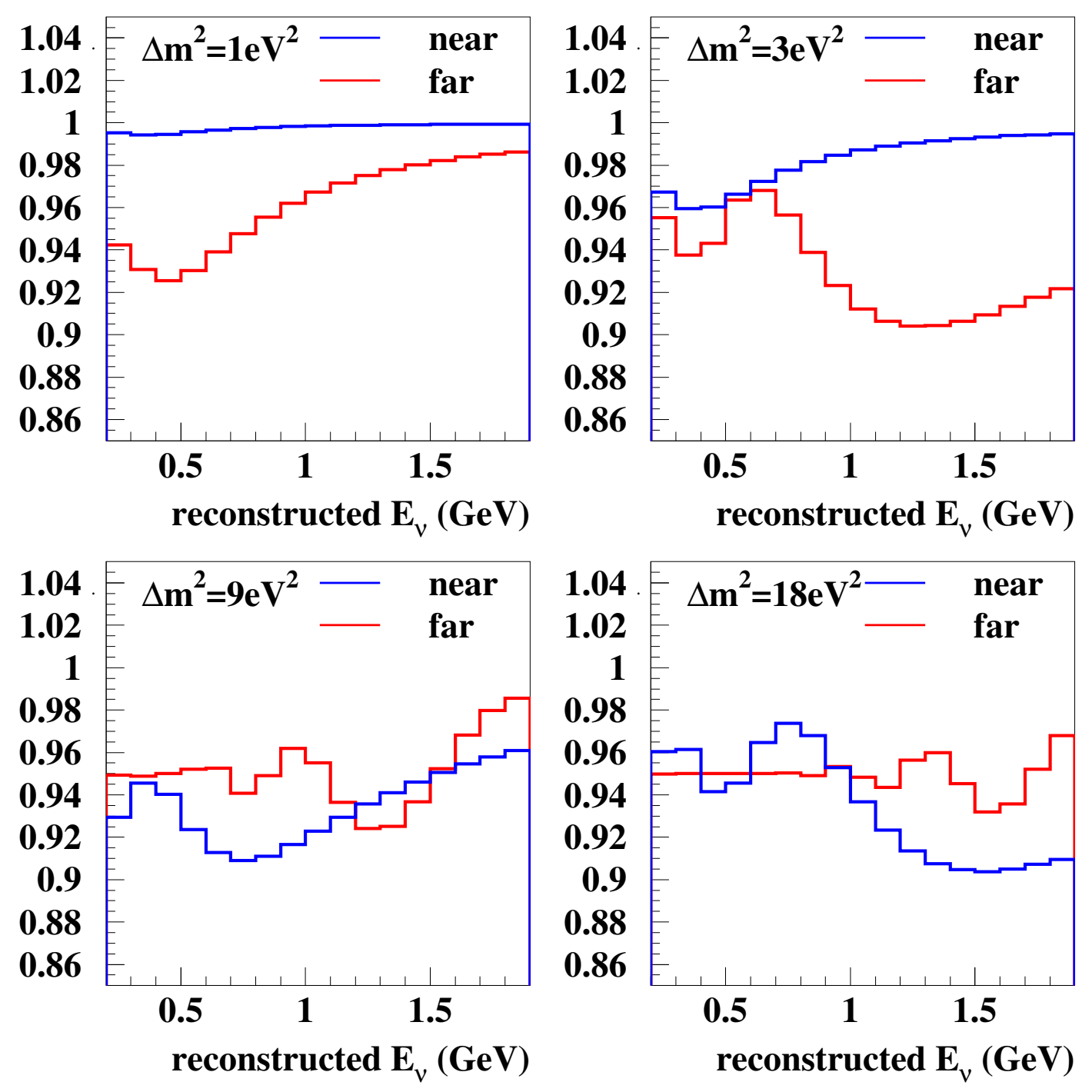

Figure 8.5: The ratio of an oscillated spectrum to an unoscillated spectrum vs reconstructed neutrino energy. Oscillation scenarios shown correspond to $\sin ^{2}(2 \theta)=0.1$, and $\Delta m^{2}=1 \mathrm{eV}^{2}$ (top left), $\Delta m^{2}=3 \mathrm{eV}^{2}$ (top right), $\Delta m^{2}=9 \mathrm{eV}^{2}$ (bottom left) and $\Delta m^{2}=18 \mathrm{eV}^{2}$ (bottom right). The near and far detector both correspond to MiniBooNE, with the near detector at $100 \mathrm{~m}$ (blue) and the far detector at $541 \mathrm{~m}$ (red). 


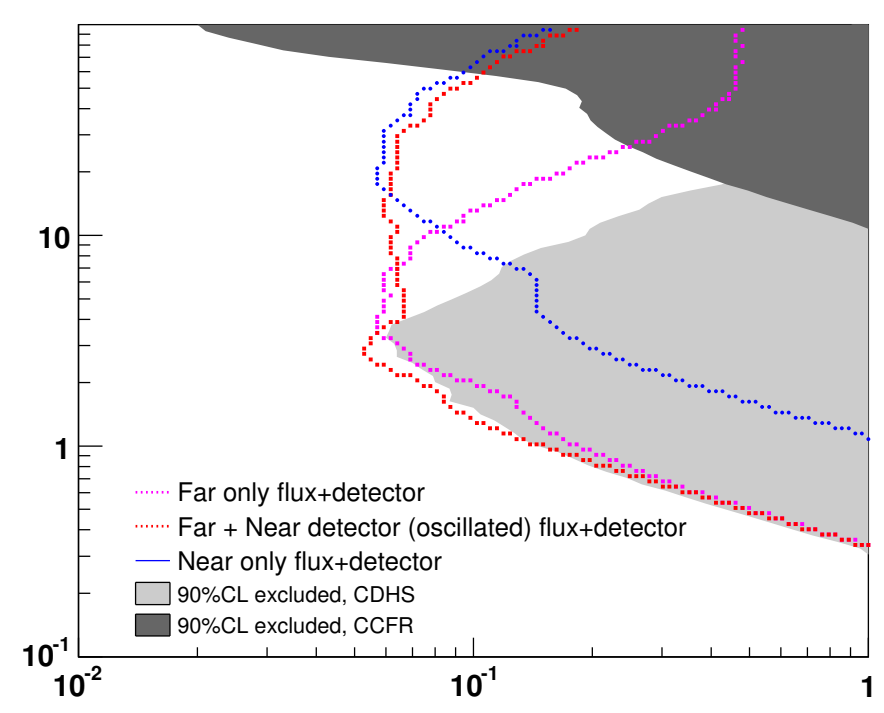

Figure 8.6: The 90\% CL sensitivity for: a single 16 bin far detector fit using flux and detector uncertainties (pink), a joint near+far detector 32 bin fit using flux and detector uncertainties (red), and a single near detector 16 bin fit using flux uncertainties. The near detector is allowed to oscillate. All fits include shape and normalization information. 

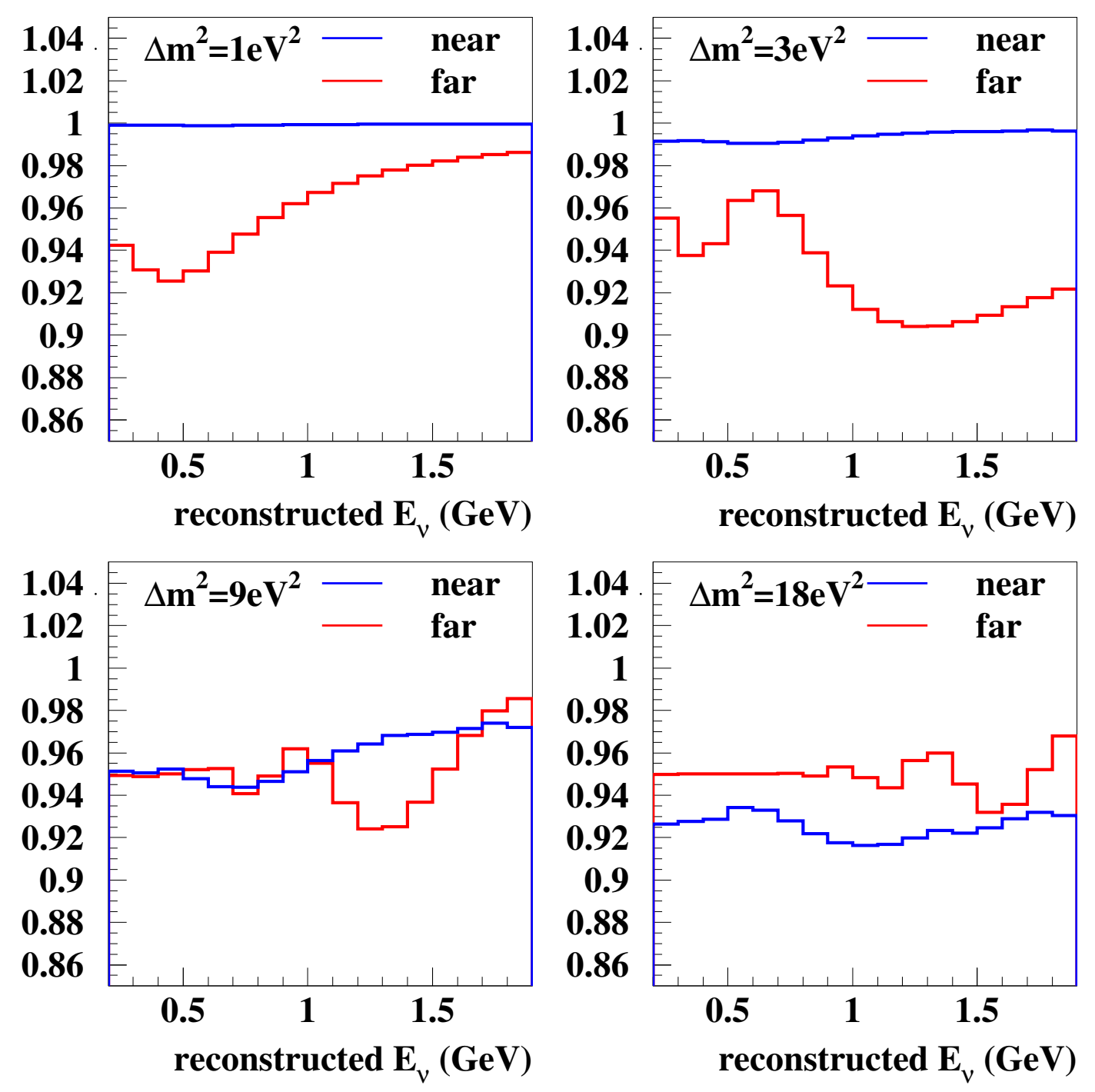

Figure 8.7: The ratio of an oscillated spectrum to an unoscillated spectrum vs reconstructed neutrino energy. Oscillation scenarios shown correspond to $\sin ^{2}(2 \theta)=0.1$, and $\Delta m^{2}=1 \mathrm{eV}^{2}$ (top left), $\Delta m^{2}=3 \mathrm{eV}^{2}$ (top right), $\Delta m^{2}=9 \mathrm{eV}^{2}$ (bottom left) and $\Delta m^{2}=18 \mathrm{eV}^{2}$ (bottom right). The near detector corresponds to the SciBooNE CC inclusive sample neutrino spectrum (blue) and the far detector corresponds to MiniBooNE (red). 


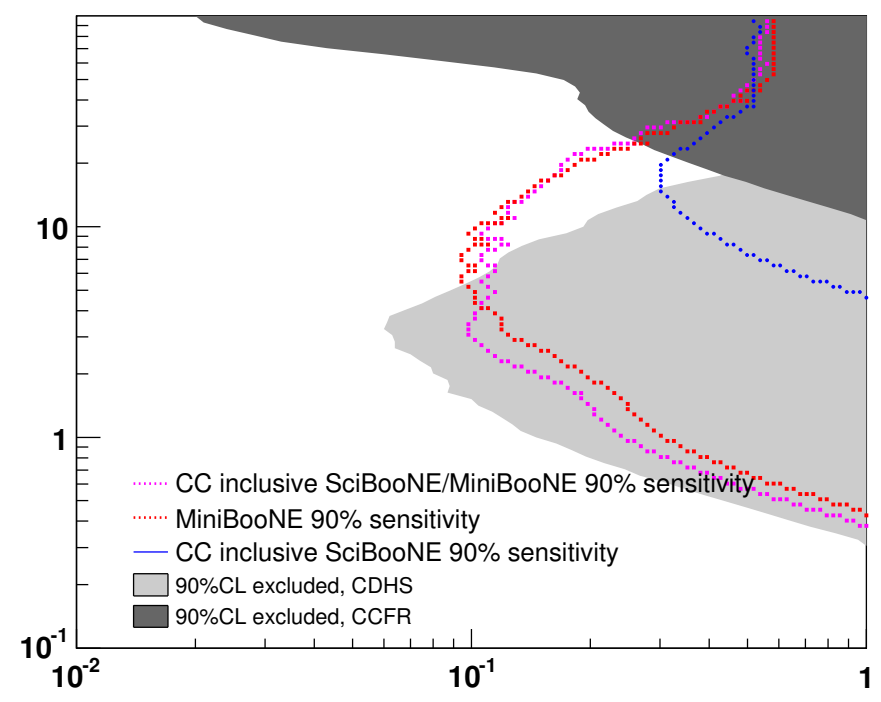

Figure 8.8: The 90\% CL sensitivity for a 32 bin fit to MiniBooNE and SciBooNE CC inclusive sample (MRD stopped events). Flux, cross section, detector and statistical uncertainties are included. MiniBooNE corresponds to 5.579e20, and SciBooNE to 0.98e20. Both samples are allowed to oscillate.

The $90 \%$ CL sensitivity with the SciBooNE CC inclusive sample included is shown in Fig. 8.8. SciBooNE constrains MiniBooNE at low energy, but the joint sensitivity is covered by the exclusion region of CDHS. At high energy, the CC inclusive-only disappearance limit does not improve over the MiniBooNE only measurement.

\subsection{Improvements to the Joint $\nu_{\mu}$ Analysis}

The dominant effect at low $\Delta m^{2}$ in the joint sensitivity is the MiniBooNE detector uncertainties. The MiniBooNE detector uncertainties are difficult to improve further, and so with the CDHS measurement already excluding the region where the SciBooNE constraint is strongest, it is unlikely that the low $\Delta m^{2}$ region will improve with a joint measurement. 
At high $\Delta m^{2}$, the sensitivity is effectively a SciBooNE-only measurement. To improve this sensitivity, one can investigate the addition of a SciBar-only CC inclusive sample and an EC stopped sample. A second improvement might be to increase the CCQE purity in the MRD sample used for this thesis. However, with 1e20 POT taken in neutrino mode, the tradeoff between statistical error and purity must be investigated carefully.

\subsection{Prospects for the Joint $\bar{\nu}_{\mu}$ Analysis}

The addition of SciBooNE samples benefits the analysis most at low $\Delta m^{2}$. As the CDHS experiment did not make a measurement in antineutrino mode, any gains to the antineutrino mode analysis are a direct improvement over the MiniBooNE-only result. In addition, MiniBooNE is currently taking data in antineutrino mode and will have collected an estimated $\sim 5 \times 10^{20}$ POT in antineutrino mode by June 2009 which can also be incorporated in a joint analysis. 


\section{Chapter 9}

\section{Conclusion}

A search for $\nu_{\mu}$ and $\bar{\nu}_{\mu}$ disappearance has been performed in the $\Delta m^{2}$ region of a few $\mathrm{eV}^{2}$ with the MiniBooNE detector. No disappearance is observed in the shape-only fit at $90 \% \mathrm{CL}$, which covers new parameter space not previously measured by CDHS and CCFR, as shown in Fig. 9.1. The data and prediction agree with each other for both neutrino mode (Fig. 6.16) and antineutrino mode (Fig. 7.13). In addition, the results constrain $3+2$ sterile neutrino models, as suggested by the exclusion of a particular $3+2$ model. The $\bar{\nu}_{\mu}$ disappearance measurement is the first ever below $10 \mathrm{eV}^{2}$. Appendix D contains the draft of these results which has been submitted to Physical Review Letters.

The potential for a $\nu_{\mu}$ disappearance analysis using both SciBooNE and MiniBooNE was also explored. Unfortunately, the region of parameter space where the constraint from SciBooNE is the strongest is already excluded by the CDHS experiment in neutrino mode. An antineutrino joint analysis should supersede the MiniBooNE-only $\bar{\nu}_{\mu}$ disappearance result discussed in this thesis, furthering our understanding of CPT violation in the neutrino sector. 


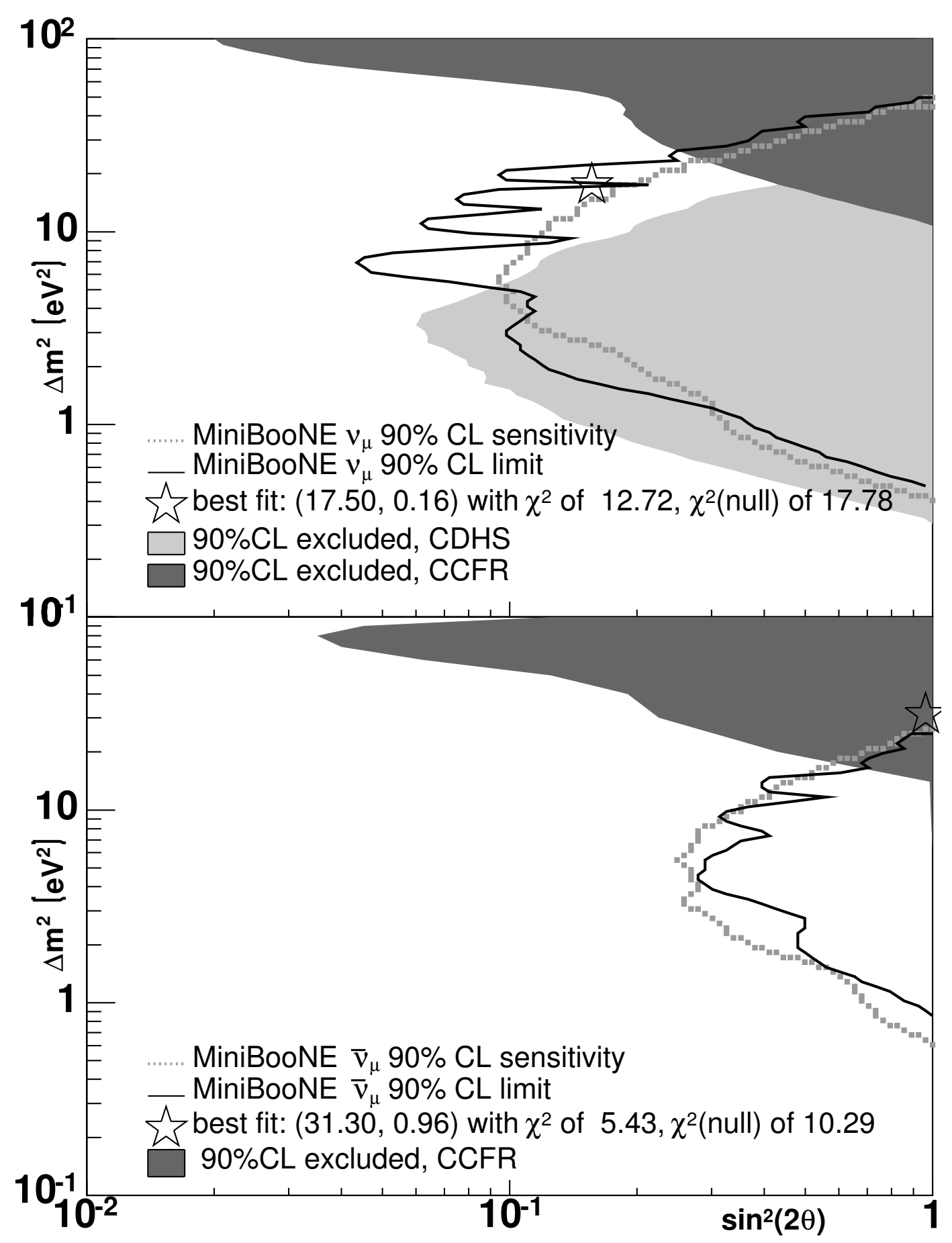

Figure 9.1: The top plot shows the sensitivity (dashed line) and limit (solid line) for 90\% CL for neutrino disappearance in MiniBooNE. Previous limits by CCFR (dark grey) and CDHS (light grey) are also shown. The bottom plot uses the same convention for antineutrino disappearance. 


\section{Appendix A}

\section{MiniBooNE $E_{\nu}^{Q E}$ calculation}

The following discussion uses a selection of contained, CCQE events, veto hits $<6$, main hits $>100,2$ subevents, and reconstructed radius $r<500 \mathrm{~cm}$.

The first correction is applied by fitting a profile histogram of the true muon energy as compared to the reconstructed value. The reconstructed muon kinetic energy, $T_{\mu}^{c o r r}$, is corrected with a linear fit:

$$
T_{\mu}^{c o r r}=a_{\mu} T_{\mu}+b_{m u}
$$

where $T_{\mu}$ is the reconstructed muon energy in $\mathrm{GeV}$, and $\left(a_{\mu}, b_{\mu}\right)=(0.8867,0.0927)$ are the fitted correction. Fig. A.1 shows the result of this correction. No correction is applied to the muon angle, as good linearity is observed (Fig. 5.5).

The reconstructed energy for the neutrino is then calculated using Eq. 5.1 and $E_{\mu}=$ $T_{\mu}^{c o r r}+m_{\mu} \cdot Q^{2}$ is also calculated, according to:

$$
Q^{2}=-m_{\mu}^{2}+2 E_{\nu}^{Q E}\left(E_{\mu}-p_{\mu} \cos \theta_{\mu}\right)
$$

The reconstructed neutrino energy $\left(E_{\nu}^{Q E}\right)$ and its comparison to true neutrino energy $\left(E_{\nu}\right)$ at this stage is plotted in Fig. A.2. Fig. A.3 shows the difference between $E_{\nu}^{Q E}$ and $E_{\nu}$ versus $Q^{2}$ and $E_{\nu}$.

Already there is a linear relationship between $E_{\nu}$ and $E_{\nu}^{Q E}$, however, there is a trend visible in $Q^{2}$, which is due to Fermi smearing. For a given true neutrino energy, the outgoing lepton's energy will distributed according to the state of the nucleon and "smeared" out; for a RFG model, this is expected to be parabolic. 


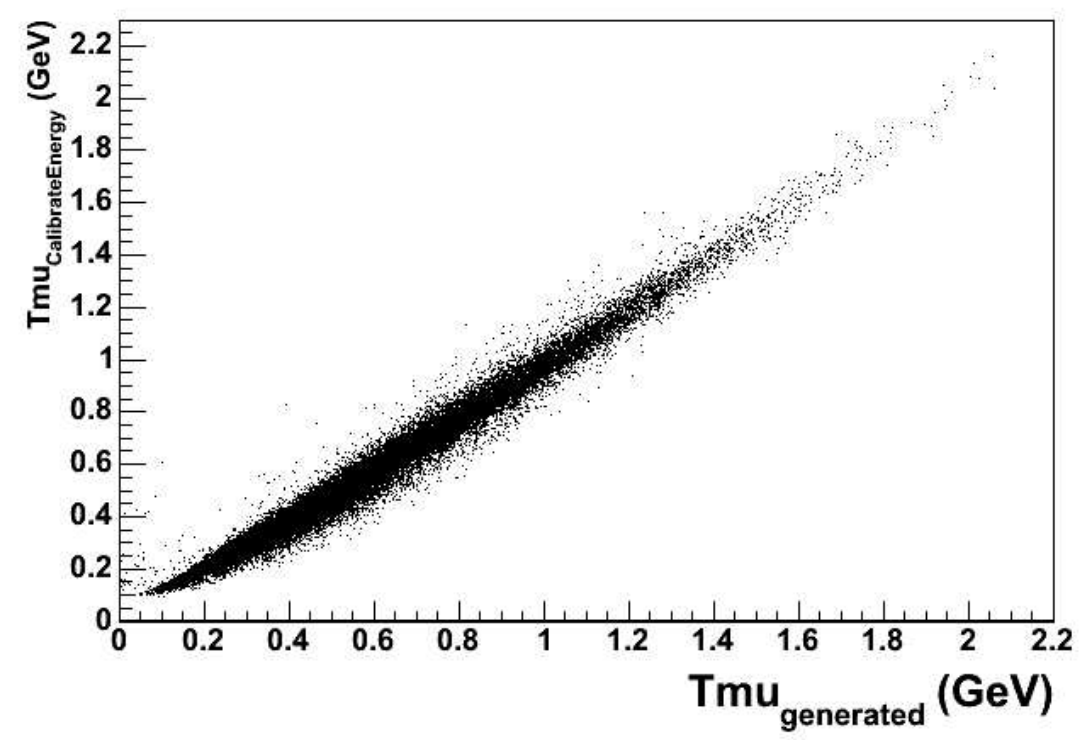

Figure A.1: Reconstructed versus true muon kinetic energy after the first correction is applied.

To correct for the Fermi-smearing, the neutrino energy is fit in $Q^{2}$ with 3rd order polynomial:

$$
E_{\nu}^{Q E}=E_{\nu}^{Q E}-\left[a_{0}+a_{1} Q^{2}+a_{2}\left(Q^{2}\right)^{2}+a_{3}\left(Q^{2}\right)^{3}\right]
$$

with $\left(a_{0}, a_{1}, a_{2}, a_{3}\right)=(-0.0777,0.1189,0.1777,-0.0291)$ as the fitted parameters.

The effect of this correction can be seen in Fig. A.4. A residual difference remains between $E_{\nu}$ and $E_{\nu}^{Q E}$, this is corrected in the final step using a quadratic polynomial between $0.2-2.0 \mathrm{GeV}$, and $E_{\nu}^{Q E}$ is calculated according to:

$$
E_{\nu}^{Q E}=\frac{\sqrt{\left(1+b_{1}\right)^{2}+4 b_{2}\left(E_{\nu}^{Q E}-b_{0}\right)}-\left(1+b_{1}\right)}{2 b_{2}}
$$

where $\left(b_{0}, b_{1}, b_{2}\right)=(0.1986,-0.3144,0.0911)$ are the fit parameters. 

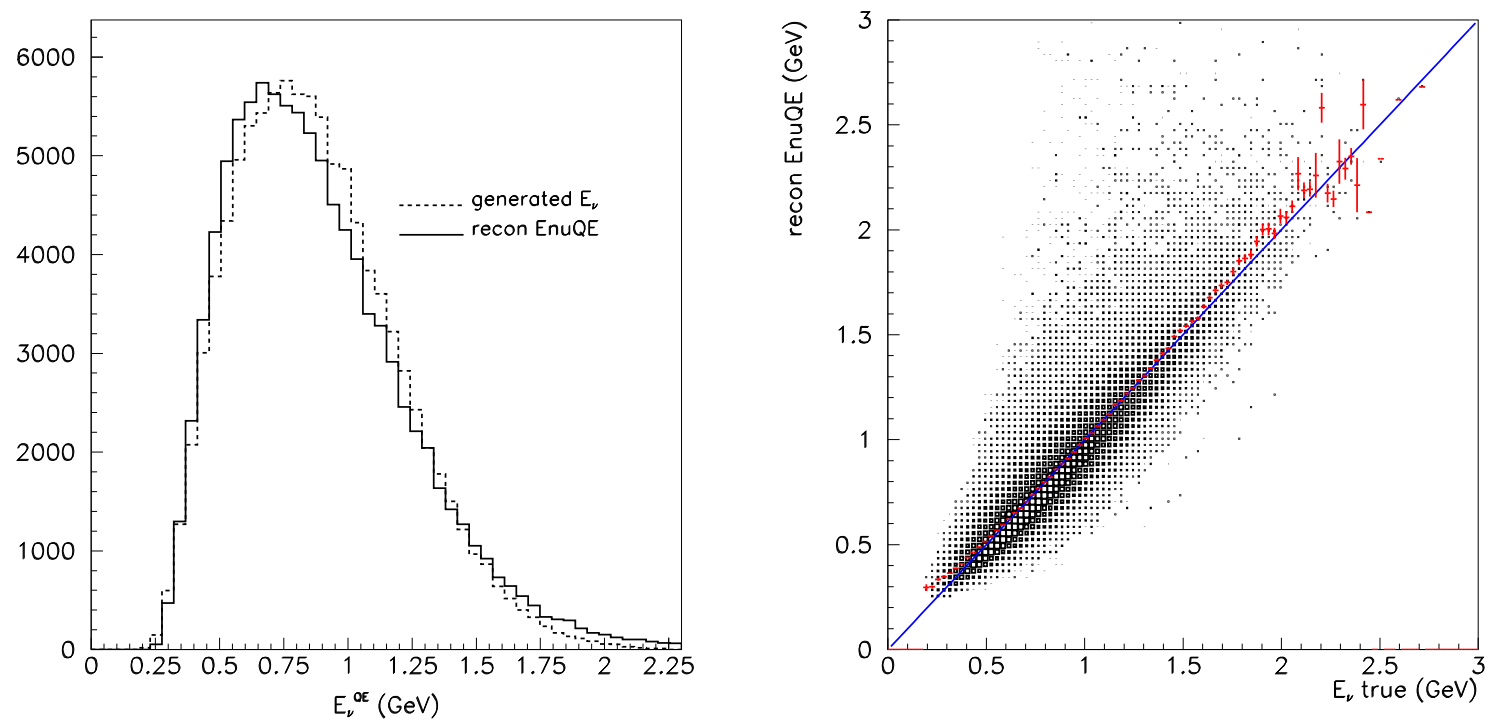

Figure A.2: Left: comparison of reconstructed $E_{\nu}^{Q E}$ (solid) and generated $E_{\nu}$ (dashed). Right: reconstructed $E_{\nu}^{Q E}$ vs. generated $E_{\nu}$. The red points are a profile plot; the blue line is at $y=x$.
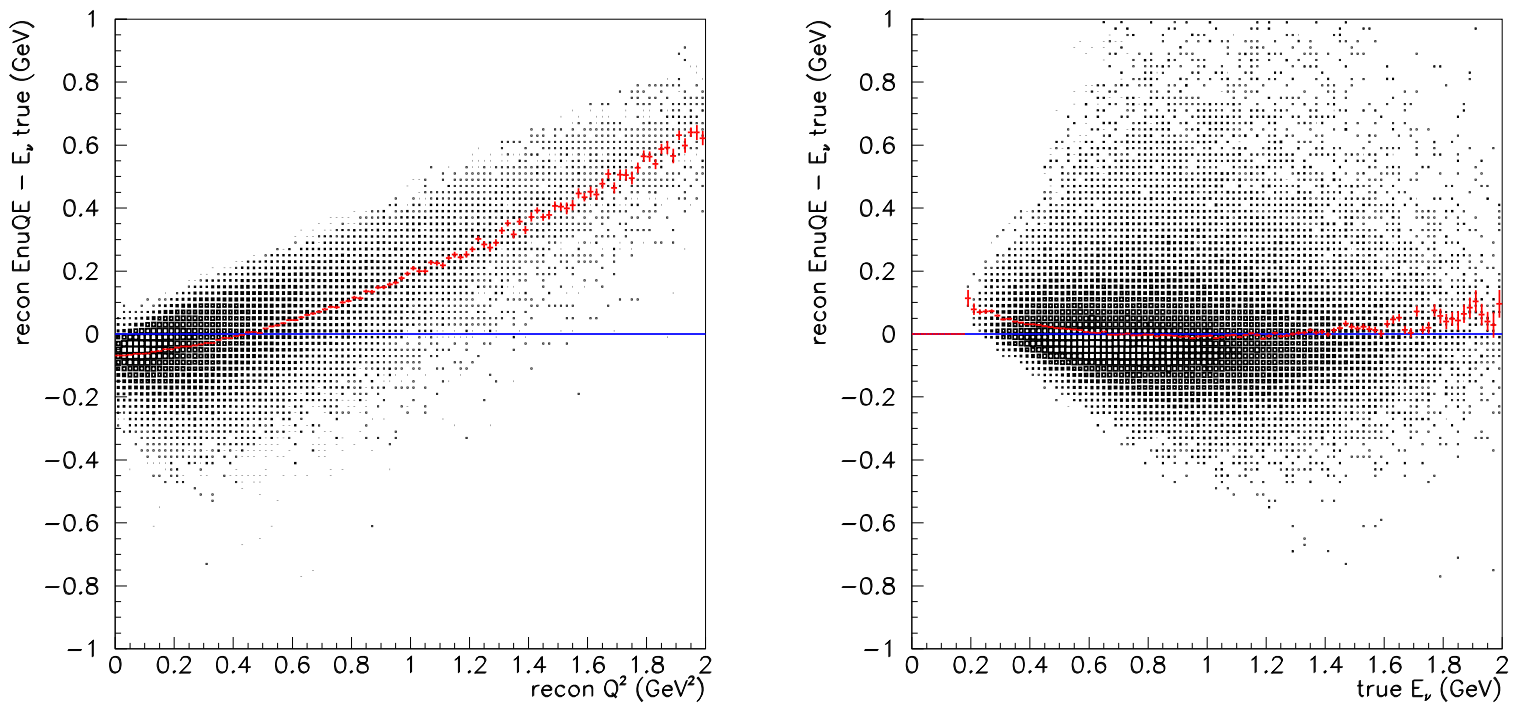

Figure A.3: Left: Difference in reconstructed $E_{\nu}^{Q E}$ minus generated $E_{\nu}$ versus $Q^{2}$ Right: Difference in reconstructed $E_{\nu}^{Q E}$ minus generated $E_{\nu}$ versus generated $E_{\nu}$. The red points are a profile plot. 

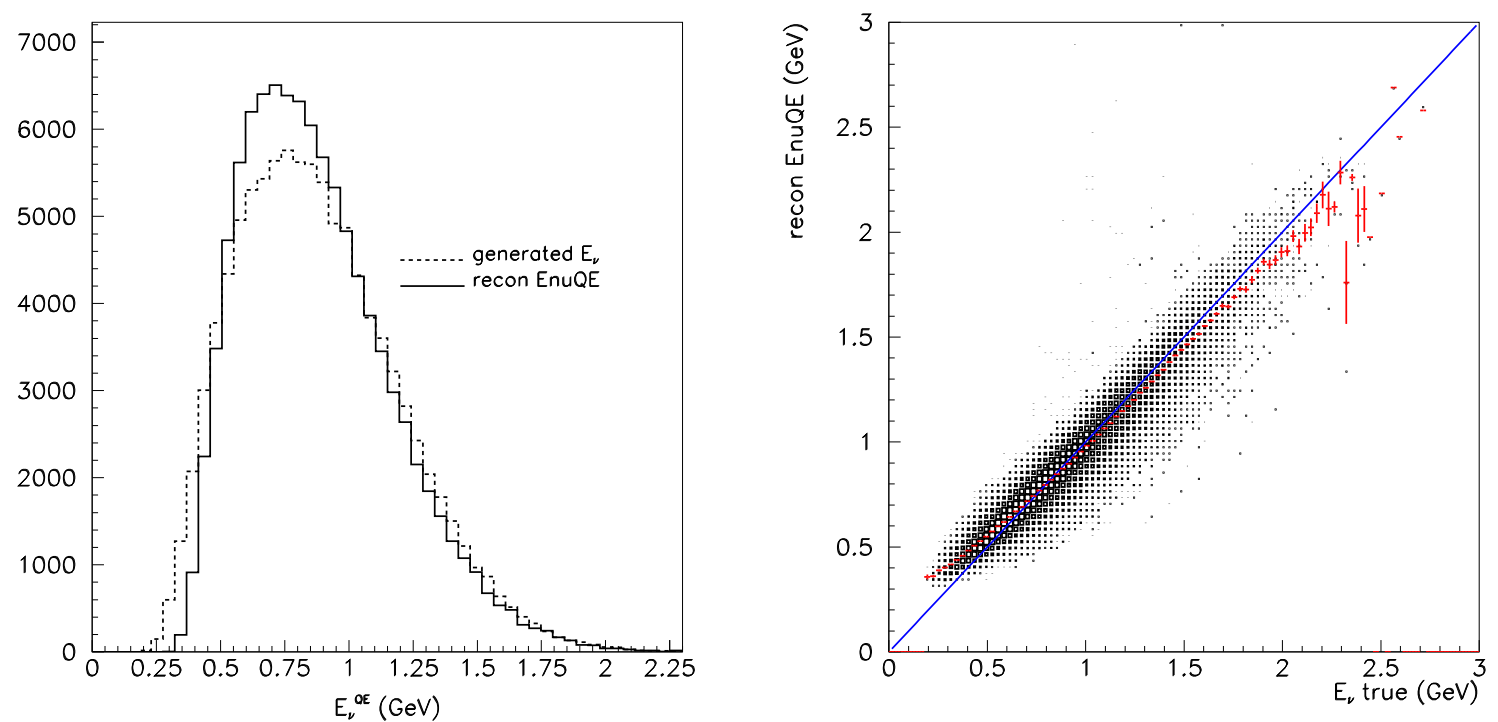

Figure A.4: Left: comparison of reconstructed $E_{\nu}^{Q E}$ (solid) and generated $E_{\nu}$ (dashed). Right: reconstructed $E_{\nu}^{Q E}$ vs. generated $E_{\nu}$. The red points are a profile plot; the blue line is at $y=x$. 


\section{Appendix B}

\section{Shape-only Error Matrices}

This section checks the form for the shape error matrix (Eq. 6.10) on simple examples. In addition, the last example shows that the shape error matrix is equivalent to building an error matrix with the draws normalized to the same value. The shape-only error matrix form is derived in Ref. [118].

\section{B.1 Shape-only Matrix Generation for Simple Cases}

Consider a two bin case: $p_{1}=a, p_{2}=b$, and a fractional error $A$.

\section{B.1.1 Normalization Error Matrix}

A fully correlated normalization error has the same fractional error in every bin:

$$
M_{i, j}^{n o r m}=\left[\begin{array}{cc}
A a^{2} & A a b \\
A a b & A b^{2}
\end{array}\right]
$$

The first element of the shape error matrix generated from a pure normalization error matrix, calculated from Eq. 6.10:

$$
\begin{aligned}
M_{11} & =M_{11}^{\text {norm }}-\frac{a}{a+b}\left(M_{11}^{\text {norm }}+M_{12}^{\text {norm }}\right)-\frac{a}{a+b}\left(M_{11}^{\text {norm }}+M_{21}^{\text {norm }}\right) \\
& +\frac{a^{2}}{(a+b)^{2}}\left(M_{11}^{\text {norm }}+M_{12}^{\text {norm }}+M_{21}^{\text {norm }}+M_{22}^{\text {norm }}\right) \\
M_{11} & =A a^{2}-\frac{2 A a^{2}}{a+b}(a+b)+\frac{a^{2}}{(a+b)^{2}} A\left(a^{2}+b^{2}+2 a b\right) \\
& =0
\end{aligned}
$$


Similar cancellations occur for all other elements, and, as desired, a pure normalization error matrix produces no shape error with Eq. 6.10.

\section{B.1.2 Shape-only Error Matrix}

Now test a pure shape error matrix with $\mathrm{N}$ events shifting between the two bins:

$$
M_{i, j}^{\text {shape }}=\left[\begin{array}{cc}
N & -N \\
-N & N
\end{array}\right]
$$

The first element of the shape error matrix:

$$
\begin{aligned}
M_{11} & =M_{11}^{\text {shape }}-\frac{a}{a+b}\left(M_{11}^{\text {shape }}+M_{12}^{\text {shape }}\right)-\frac{a}{a+b}\left(M_{11}^{\text {shape }}+M_{21}^{\text {shape }}\right) \\
& +\frac{a^{2}}{(a+b)^{2}}\left(M_{11}^{\text {shape }}+M_{12}^{\text {shape }}+M_{21}^{\text {shape }}+M_{22}^{\text {shape }}\right) \\
M_{11} & =N-0-0+0 \\
& =N
\end{aligned}
$$

All other elements return the original matrix, as sums over rows or columns sum to 0. One can also calculate this example using fractional errors, but the total number of events shifting between bins must be the same.

\section{B.1.3 Shape+Normalization Case}

Consider shape and normalization error on two bins, where $a, b=(20,10)$, with a shape error migrating 1 event from bin 1 to bin 2, and a normalization uncertainty of $10 \%$ :

$$
M_{i, j}^{\text {combo }}=\left[\begin{array}{cc}
1 & -1 \\
-1 & 1
\end{array}\right]+\left[\begin{array}{ll}
4 & 2 \\
2 & 1
\end{array}\right]=\left[\begin{array}{ll}
5 & 1 \\
1 & 2
\end{array}\right]
$$

The shape only error matrix calculation gives:

$$
M_{i, j}^{c o m b o}=\left[\begin{array}{cc}
1 & -1 \\
-1 & 1
\end{array}\right]
$$

An alternate way to generate the shape error matrix would be to use Eq. 6.11, when the fluctuation in question is renormalized to the same total number of events. The fluctuation, $p^{\delta}=(21,12)$ is compared to $p^{\alpha}=(20,10)$ to form an error matrix. First, normalizing 
$p^{\delta}$ to the same number of events as $p^{\alpha}$ gives: $p^{\delta}=(19,11)$. Then the error matrix is formed:

$$
\begin{aligned}
M_{1,1}^{\text {shape }} & =\left(p_{1}^{\alpha}-p_{1}^{\delta}\right)\left(p_{1}^{\alpha}-p_{1}^{\delta}\right) \\
& =(21-20)^{2}=1 \\
M_{1,2}^{\text {shape }} & =\left(p_{1}^{\alpha}-p_{1}^{\delta}\right)\left(p_{1}^{\alpha}-p_{1}^{\delta}\right) \\
& =(21-20)(10-11)=-1
\end{aligned}
$$

which also returns the shape error matrix. 


\section{Appendix C}

\section{Forming Error Matrices: Number of}

\section{Draws}

This section explores how many draws are necessary to resolve the shape (uncorrelated uncertainties) and the normalization (fully correlated) uncertainties with a simple example.

Consider a 15 bin matrix with uncorrelated error and normalization components, specifically a fractional error matrix $M^{\text {frac }}=M^{\text {shape }}+M^{\text {norm }}$, where $M^{\text {shape }}$ corresponds to a $1 \%$ uncorrelated error matrix, and $M^{\text {norm }}$ is a fully correlated normalization (25\%) error matrix.

The choice of $25 \%$ and $1 \%$ are to provide an extreme example of small shape errors and strong correlations; the total normalization uncertainty for the MiniBooNE error matrix is $20 \%$, with a shape error of $5-20 \%$ across energy bins.

$$
\begin{gathered}
M_{i j}^{\text {shape }}=\begin{array}{c}
0.0001 \text { if } i=j \\
0.0 i f i \neq j
\end{array} \\
M_{i j}^{\text {norm }}=0.0001 \text { for all } i, j
\end{gathered}
$$

From the fractional error matrix. construct the total error matrix, $M^{t o t a l}=M_{i j}^{f r a c} p_{i} p_{j}$, where $p_{i}$ is the default MiniBooNE prediction in 15 bins (but any prediction will suffice).

Draw $p_{i}^{k}$ from $M_{i j}$, where $k=1, n$. Fluctuate each bin according to a Gaussian with mean 0 and variance of 1 , and multiply it by the lower triangular matrix generated from 
$M^{\text {total }}$ to create a fluctuation for each bin, $\Delta_{i}^{k}$. Add the fluctuation to the prediction $p_{i}^{k}=$ $p_{i}^{c v}+\Delta_{i}^{k}$ to form a fluctuation consistent with $M$; this method is detailed in Ref. [115].

Build a new error matrix over $n$ draws:

$$
M_{i j}^{n}=\sum_{k=1}^{n}\left(p_{i}^{c v}-p_{i}^{k}\right)\left(p_{j}^{c v}-p_{j}^{k}\right)
$$

Three quantities are important for the error matrix: the normalization, the shape uncertainty, and the correlations. Each are considered as a function of numbers of draws used.

The fractional normalization (Eq. 6.9) is plotted as a function of $n$ total draws in Fig. C.1. 10 independent draws of $n=10$ are compared to 4 independent draws with $n=100$, and 2 draws with 500. In addition, the normalization is shown when all 1000 draws are used compared to the true normalization of $25 \%$. In addition, the mean and standard deviation are shown for the first three cases. Even using 10 draws for a $15 \times 15$ bin matrix produces a not unreasonable estimate of the true normalization, and using 1000 draws correctly predicts the normalization uncertainty at the $1 \%$ level.

The shape error matrix is also calculated (Eq. 6.10), and the diagonal elements plotted as a function of bin number for the sets of 10 draws, 100 draws, 500 draws and 1000 draw in Fig. C.2. As expected, the $25 \%$ normalization error is not present in the shape, so this tests the ability to resolve the $1 \%$ uncorrelated error on the diagonals. The matricies which use 10 draws calculate a shape uncertainty of $\sim 2 \%$, but the spread in even the 100 draw case is substantially improved, and the 1000 draw case is correct on the diagonals also at the $1 \%$ level. As the statistical uncertainty added to the MiniBooNE error matrix varies between $0.6 \%$ and $3 \%$, even matricies using 100 draws are sufficiently similar.

The best way to represent the correlations in the error matrix is to use a $\chi^{2}$ which sums over all elements in the error matrix:

$$
\chi^{2}=\sum_{i, j}^{15}\left(p_{i}^{c v}-p_{i}^{k=1}\right) M_{i j}^{-1}\left(p_{j}^{c v}-p_{j}^{k=1}\right)
$$

The first draw is used, the change in $\chi^{2}$ relative to number of draws, not the absolute $\chi^{2}$ is the purpose of the study. The $\chi^{2}$ as a function of number of draws is shown in 


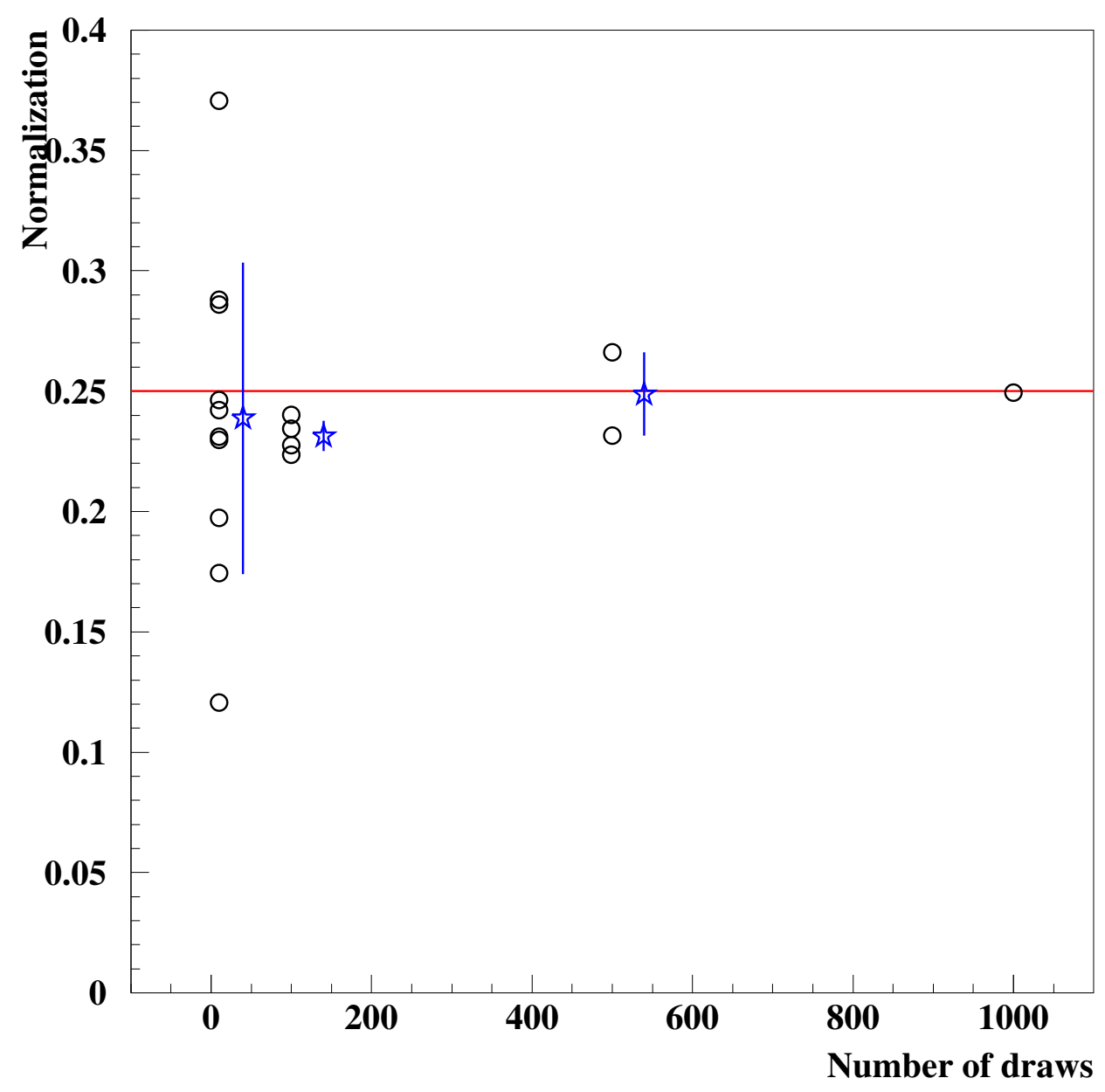

Figure C.1: Normalization for an error matrix calculated using $n$ independent draws is plotted vs. $n$. 10 draws are used to form 10 matrices, 100 draws are used to form 4 matrices and 500 draws are used to form 2 matrices, all shown with circles. The mean for each set is shown with a blue star, and the standard deviation is shown with a blue line. The normalization of the underlying matrix used to generate the draws is shown with a red line, and the matrix formed with all 1000 draws is also shown. 


\section{Shape uncertainty}

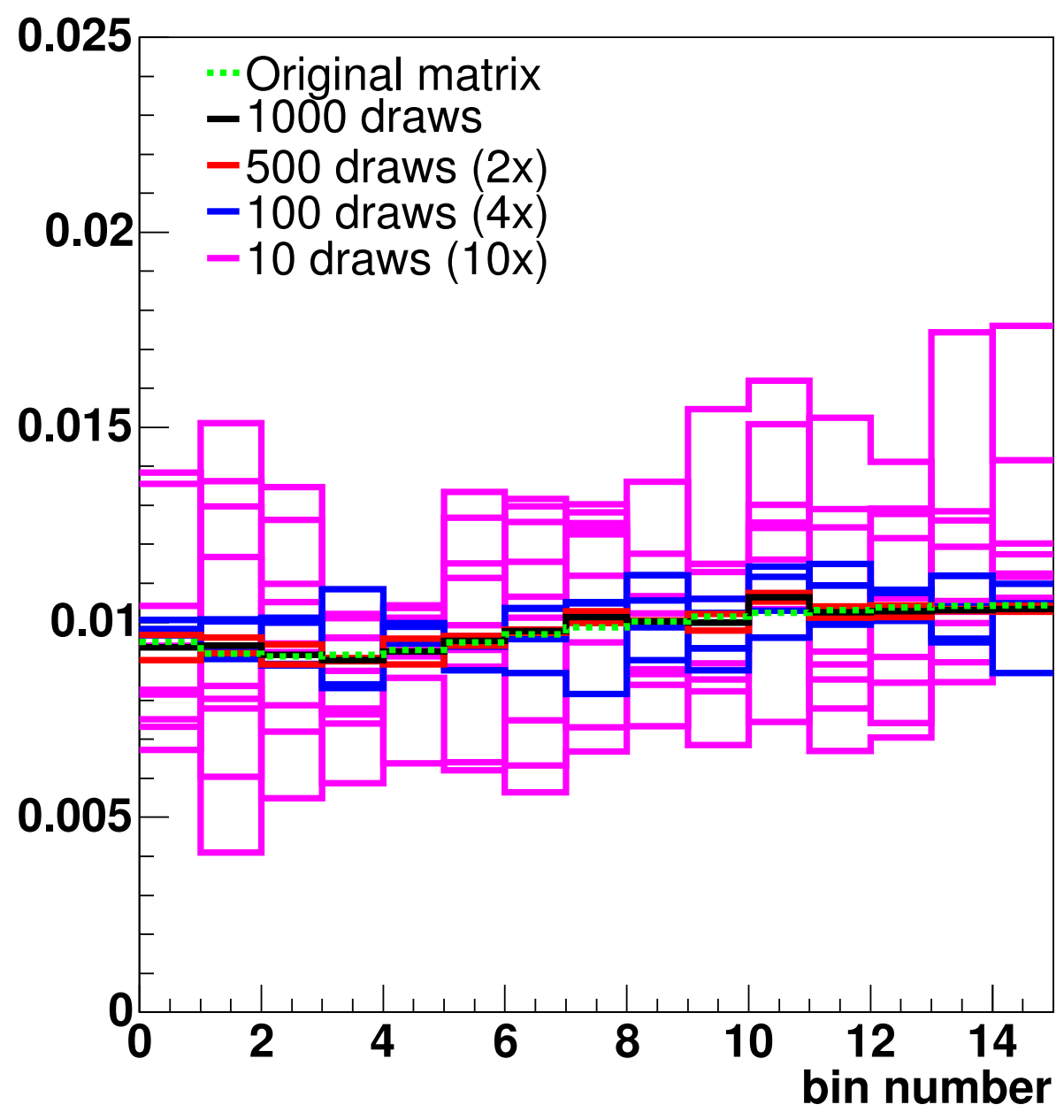

Figure C.2: Diagonal fractional shape uncertainty calculated for various matrices vs. bin number. 10 draws are used to form 10 matrices (pink), 100 draws are used to form 4 matrices (blue) and 500 draws are used to form 2 matrices (red). The original matrix's diagonal elements are shown (green) along with the matrix formed with all 1000 draws (black). 
Fig. C.3. The 10 draw $\chi^{2}$ cases are not shown, as they give a abysmal $\chi^{2}$. While the 1000 draw case does not exactly re-create the correlations, the difference in probability for the $\chi^{2} / \mathrm{DOF}=15$ between the original matrix and the one generated with 1000 draws is $0.5 \%$. Based on these studies, 1000 draws is used whenever possible to generate error matrices. 


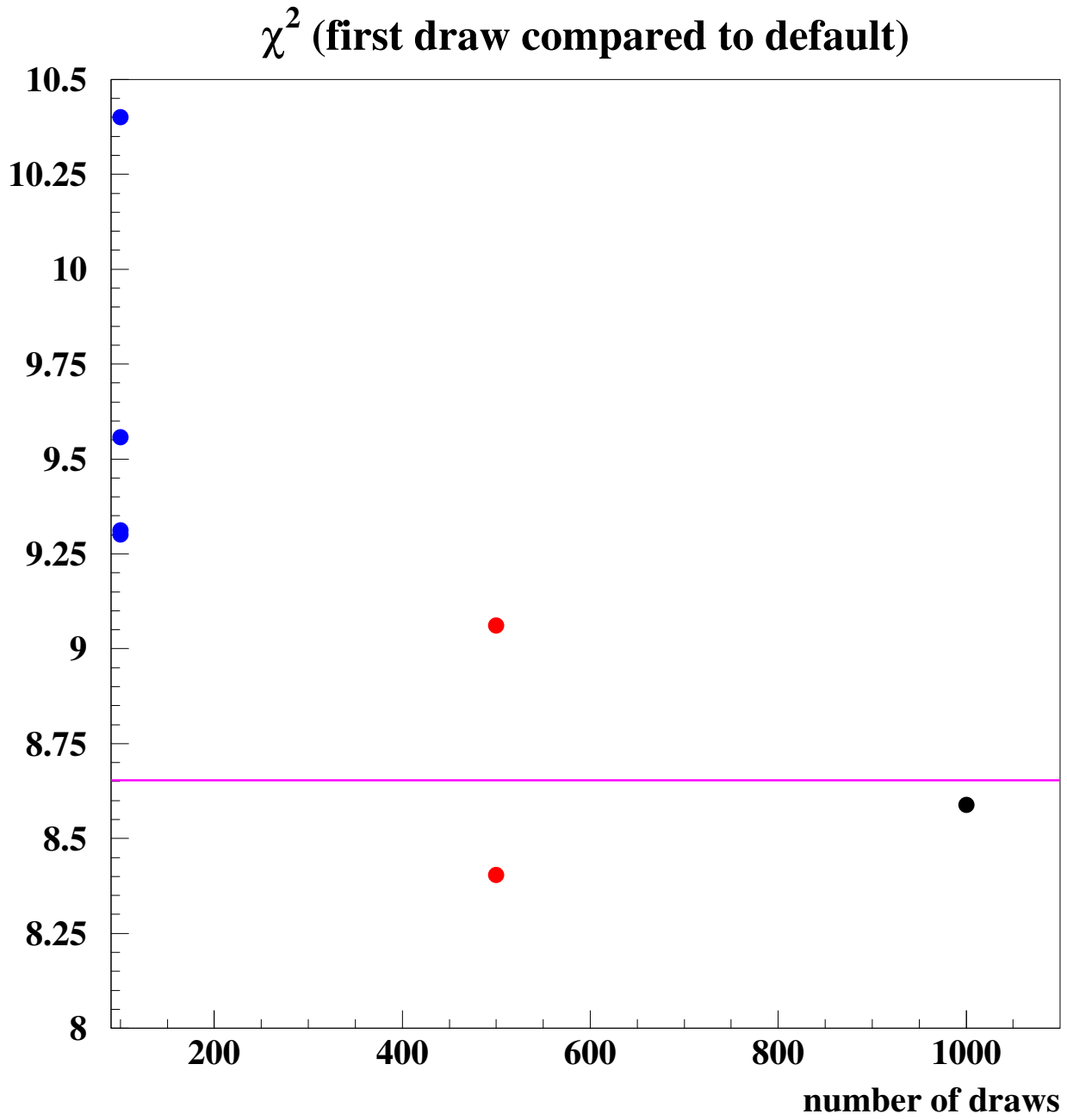

Figure C.3: The $\chi^{2}$ calculated using an error matrix with $n$ draws is shown for 4 sets of 100 draws (blue), 2 sets of 500 draws (red), and one set including all 1000 draws (black) as compared to the original matrix $\chi^{2}$ (pink line). 


\section{Appendix D}

\section{Draft MiniBooNE $\nu_{\mu}$ and $\bar{\nu}_{\mu}$ Disappearance Publication}

The following appendix contains the paper of this thesis work submitted for publication. 


\section{A search for muon neutrino and antineutrino disappearance in MiniBooNE}

A. A. Aguilar-Arevalo ${ }^{14}$, C. E. Anderson ${ }^{19}$, A. O. Bazarko ${ }^{16}$, S. J. Brice ${ }^{7}$, B. C. Brown ${ }^{7}$, L. Bugel ${ }^{5}$, J. Cao ${ }^{15}$, L. Coney ${ }^{5}$, J. M. Conrad ${ }^{5,13}$, D. C. Cox $^{10}$, A. Curioni ${ }^{19}$, Z. Djurcic ${ }^{5}$, D. A. Finley ${ }^{7}$, B. T. Fleming ${ }^{19}$, R. Ford ${ }^{7}$, F. G. Garcia ${ }^{7}$, G. T. Garvey ${ }^{11}$, C. Green ${ }^{7,11}$, J. A. Green ${ }^{10,11}$, T. L. Hart ${ }^{4}$, E. Hawker ${ }^{3,11}$, R. Imlay ${ }^{12}$, R. A. Johnson $^{3}$, G. Karagiorgi ${ }^{5,13}$, P. Kasper ${ }^{7}$, T. Katori ${ }^{10}$, T. Kobilarcik ${ }^{7}$, I. Kourbanis ${ }^{7}$, S. Koutsoliotas ${ }^{2}$, E. M. Laird ${ }^{16}$, S. K. Linden ${ }^{19}$,J. M. Link ${ }^{18}$, Y. Liu ${ }^{15}$, Y. Liu ${ }^{1}$, W. C. Louis ${ }^{11}$, K. B. M. Mahn ${ }^{5}$, W. Marsh ${ }^{7}$, G. McGregor ${ }^{11}$, W. Metcalf ${ }^{12}$, P. D. Meyers ${ }^{16}$, F. Mills ${ }^{7}$, G. B. Mills ${ }^{11}$, J. Monroe ${ }^{5}$, C. D. Moore ${ }^{7}$, R. H. Nelson ${ }^{4}$, V. T. Nguyen ${ }^{5,13}$, P. Nienaber ${ }^{17}$, J. A. Nowak ${ }^{12}$, B. Osmanov ${ }^{8}$, S. Ouedraogo ${ }^{12}$, R. B. Patterson ${ }^{16}$, D. Perevalov ${ }^{1}$, C. C. Polly ${ }^{9,10}$, E. Prebys ${ }^{7}$, J. L. Raaf ${ }^{3}$, H. Ray ${ }^{8,11}$, B. P. Roe ${ }^{15}$, A. D. Russell ${ }^{7}$, V. Sandberg ${ }^{11}$, R. Schirato ${ }^{11}$,

D. Schmitz ${ }^{5}$, M. H. Shaevitz ${ }^{5}$, F. C. Shoemaker ${ }^{16}$, D. Smith $^{6}$, M. Soderberg ${ }^{19}$, M. Sorel ${ }^{5}$, P. Spentzouris ${ }^{7}$, I. Stancu ${ }^{1}$, R. J. Stefanski ${ }^{7}$, M. Sung ${ }^{12}$, H. A. Tanaka ${ }^{16}$, R. Tayloe ${ }^{10}$, M. Tzanov ${ }^{4}$, R. Van de Water ${ }^{11}$, M. O. Wascko ${ }^{12 *}$, D. H. White ${ }^{11}$, M. J. Wilking ${ }^{4}$, H. J. Yang ${ }^{15}$, G. P. Zeller ${ }^{5,11}$, E. D. Zimmerman ${ }^{4}$

(The MiniBooNE Collaboration)

${ }^{1}$ University of Alabama; Tuscaloosa, AL 35487

${ }^{2}$ Bucknell University; Lewisburg, PA 17837

${ }^{3}$ University of Cincinnati; Cincinnati, OH 45221

${ }^{4}$ University of Colorado; Boulder, CO 80309

${ }^{5}$ Columbia University; New York, NY 10027

${ }^{6}$ Embry Riddle Aeronautical University; Prescott, AZ 86301

${ }^{7}$ Fermi National Accelerator Laboratory; Batavia, IL 60510

${ }^{8}$ University of Florida; Gainesville, FL 32611

${ }^{9}$ University of Illinois; Urbana, IL 61801

${ }^{10}$ Indiana University; Bloomington, IN 47405

${ }^{11}$ Los Alamos National Laboratory; Los Alamos, NM 87545

${ }^{12}$ Louisiana State University; Baton Rouge, LA 70803

${ }^{13}$ Massachusetts Institute of Technology; Cambridge, MA 02139

${ }^{14}$ Universidad National Autonoma de Mexico, Mexico, D.F., Mexico

${ }^{15}$ University of Michigan; Ann Arbor, MI 48109

${ }^{16}$ Princeton University; Princeton, NJ 08544

${ }^{17}$ Saint Mary's University of Minnesota; Winona, MN 55987

${ }^{18}$ Virginia Polytechnic Institute \& State University; Blacksburg, VA 24061

${ }^{19}$ Yale University; New Haven, CT 06520

(Dated: February 20, 2009)

The MiniBooNE Collaboration reports a search for $\nu_{\mu}$ and $\bar{\nu}_{\mu}$ disappearance in the $\Delta m^{2}$ region of a few $\mathrm{eV}^{2}$. Disappearance measurements are important for constraining models with extra types of neutrinos, extra dimensions and CPT violation. Fits to the shape of the $\nu_{\mu}$ and $\bar{\nu}_{\mu}$ energy spectra reveal no evidence for disappearance in either mode. This is the first test of $\bar{\nu}_{\mu}$ disappearance between $\Delta m^{2}=0.1-10 \mathrm{eV}^{2}$.

Neutrino oscillations have been observed and confirmed at mass splittings $\left(\Delta \mathrm{m}^{2}\right)$ of $\sim 10^{-5} \mathrm{eV}^{2}$ and $\sim 10^{-3} \mathrm{eV}^{2}$, called the "solar" and "atmospheric" oscillations respectively. The mixing observed is consistent with three generations of neutrinos and a unitary mixing matrix. The LSND experiment, however, observed an excess of $\bar{\nu}_{e}[1]$ in a $\bar{\nu}_{\mu}$ beam, indicating a possible third $\Delta \mathrm{m}^{2}$ around $1 \mathrm{eV}^{2}$ that would require more than three neutrino generations or other exotic physics. Recently, the MiniBooNE experiment[2] excluded at the $98 \%$ Con-

*Present address: Imperial College; London SW7 2AZ, United Kingdom fidence Level (CL) two neutrino appearance-only oscillations as an explanation of the LSND excess if oscillations of neutrinos and antineutrinos are the same.

Exotic physics models[3-6], including sterile neutrinos, extra dimensions, and CPT violation have been proposed to explain the LSND results. Some of these models can accommodate the MiniBooNE $\nu_{e}$ appearance oscillation results. These models are testable with measurements of $\nu_{\mu}$ and $\bar{\nu}_{\mu}$ disappearance which constrain any nonstandard oscillations of $\stackrel{(-)}{\nu_{\mu}} \rightarrow \stackrel{(-)}{\nu_{x}}$. As described in this article, the MiniBooNE experiment has performed searches for $\nu_{\mu}$ and $\bar{\nu}_{\mu}$ disappearance which probe a region of interest, $\Delta m^{2}$ range $0.5-40 \mathrm{eV}^{2}$, not covered by two previous disappearance experiments, CCFR [7] $\left(\nu_{\mu}\right.$ and 
$\left.\bar{\nu}_{\mu}\right)$ and CDHS [8] ( $\nu_{\mu}$ only).

For the MiniBooNE experimental setup, the detector is located at a fixed distance from the neutrino source. In this case, $\nu_{\mu}$ disappearance due to oscillations has a distinct signature as a function of neutrino energy, because neutrinos with different energies oscillate with different probabilities for the same distance traveled. Disappearance would be observable either via a deficit of events (normalization) or, alternatively, via a distortion of the neutrino energy spectrum (shape). The absolute normalization uncertainties are large and so, for MiniBooNE, a shape-only disappearance is performed. (The ratio of detected events to predicted events in MiniBooNE for neutrinos is $1.31 \pm 0.26,1.18 \pm 0.18$ for antineutrinos, which shows agreement within the uncertainties.)

The $\stackrel{(-)}{\nu_{\mu}}$ flux to the MiniBooNE detector is provided by the Fermilab Booster Neutrino Beam (BNB) which is produced by $8 \mathrm{GeV}$ protons incident on a $1 \mathrm{~cm}$ diameter $71 \mathrm{~cm}$ long (1.7 interaction length) beryllium target surrounded by a magnetic horn pulsed at $174 \mathrm{kA}$. The horn uses positive current to focus $\pi^{+}$and $K^{+}$mesons for the neutrino mode sample and negative current to focus $\pi^{-}$ and $K^{-}$for the antineutrino mode sample. The mesons that pass through a $60 \mathrm{~cm}$ diameter collimator $259 \mathrm{~cm}$ downstream of the target decay in a $50 \mathrm{~m}$ long tunnel to produce the $\nu_{\mu}^{(-)}$beam. The BNB flux [9] is simulated using GEANT4 [10]. Pion and kaon production in the target is parametrized by a global fit to proton-beryllium particle production data[9].

The distance from the proton interaction target to the MiniBooNE detector[11] is $541 \mathrm{~m}$. The MiniBooNE detector is a $12 \mathrm{~m}$ diameter spherical tank filled with 800 tons of mineral oil. The detector has an inner region with 1280 inward facing 8 inch photomultiplier tubes (PMTs), and an optically isolated outer veto region to reject cosmicray induced events. Charged particles produced in neutrino interactions emit primarily Cherenkov light, though a small amount of scintillation light is also produced.

Neutrino interactions are simulated with the v3 NUANCE event generator [12]. Approximately $42 \%$ of all events in MiniBooNE are due to charged current quasielastic (CCQE) neutrino interactions $\left(\nu_{\mu}+n \rightarrow \mu^{-}+p\right)$ and $22 \%$ are due to charged current single pion production $\left(\mathrm{CC} 1 \pi^{+/-}\right)$for both neutrino and antineutrino modes.

The search for oscillations is conducted with a sample of CCQE events because of the high statistics and purity and because the outgoing muon's energy and angle are sufficient to reconstruct the incoming neutrino energy. The reconstructed neutrino energy $\left(E_{\nu}^{Q E}\right)$ is formed assuming the target is at rest inside the nucleus, and a small correction is applied in both data and simulation to account for the biasing effects of Fermi-smearing. At 300 $\mathrm{MeV}$, the muon energy resolution is $7 \%$ and the angular resolution is 5 degrees. The average $E_{\nu}^{Q E}$ resolution is
$11 \%$ for events in the CCQE sample.[13].

CCQE events are selected by identifying a single muon in the detector and its associated decay electron, with the same criteria as was used for the previous measurement of the CCQE cross section on carbon[13]. Timing information from the PMTs allows the light produced by the initial neutrino interaction (first "subevent") to be separated from the light produced by the decay electron (second "subevent"). The timing and charge response of the PMTs is used to reconstruct the position, kinetic energy and direction vector of the primary particle within each subevent. Exactly two subevents are required (the muon and its decay electron). To reject cosmic ray interactions, both subevents are required to have fewer than 6 veto-PMT hits. The first subevent must be in coincidence with a beam pulse, have a reconstructed track center less than $500 \mathrm{~cm}$, and greater than 200 inner tank PMT hits to eliminate electrons from stopping cosmic ray muon decays. The second subevent must have less than 200 inner PMT hits to be below the decay electron energy endpoint. Finally, the distance between the electron vertex and the muon track endpoint must be less than $100 \mathrm{~cm}$, ensuring that the electron is associated with the muon track. This selection also works for the antineutrino mode sample as the final state nucleon is not reconstructed and the detector does not distinguish muon charge.

The selection yields 190,454 data events with $0<$ $E_{\nu}^{Q E}<1.9 \mathrm{GeV}$ for $5.58 \times 10^{20}$ protons on target (POT) in the neutrino mode sample; 27,053 data events for $3.39 \times 10^{20} \mathrm{POT}$ in antineutrino mode sample. The neutrino mode sample according to the simulation is $74 \%$ pure CCQE, with $<1 \% \bar{\nu}_{\mu}$ content. For antineutrino mode, the beam is not pure $\bar{\nu}_{\mu}$ but has a substantial contribution of $\nu_{\mu}$ due to the higher $\pi^{+}$production at the target and the higher $\nu_{\mu}$ cross section. According to the simulation, the antineutrino mode sample is $70 \%$ pure CCQE, with $25 \%$ coming from $\nu_{\mu}$ interactions. The primary background $(\sim 75 \%)$ for both the $\nu_{\mu}$ and $\bar{\nu}_{\mu}$ samples is $\mathrm{CC} 1 \pi$ events where the outgoing pion is unobserved due to absorption in the nucleus or low energy.

The CCQE cross section depends on the axial vector form factor, which is commonly assumed to have a dipole form as a function of $Q^{2}$ with one adjustable parameter, $M_{A}$, the axial mass. The world's data on neutrino interactions on deuterium gives $M_{A}^{H}=1.015 \mathrm{GeV}[16]$, however recent results from the K2K experiment on carbon[17] and oxygen[18] suggest a higher effective value of $M_{A}$ for nuclear targets. In a shape-only fit in $Q^{2}$, the MiniBooNE $\nu_{\mu}$ CCQE data also favors a higher value of $M_{A}^{e f f}=1.23 \mathrm{GeV}$ and a nuclear effect parameter modeling Pauli blocking, $\kappa=1.019[13]$. The effect of $M_{A}$ on the $Q^{2}$ shape is pronounced while oscillations provide relatively little $Q^{2}$ distortion. Therefore, the effect of the cross section tuning does not mask any underlying disappearance in the neutrino or antineutrino mode samples. 
For the disappearance analysis, $M_{A}$ is set to the world's data default value, $M_{A}=1.015 \mathrm{GeV}$ and the uncertainties span the difference between the more recent MiniBooNE and $\mathrm{K} 2 \mathrm{~K}$ values and deuterium-based results. The uncertainty in the $\mathrm{CC} 1 \pi$ background is based on the MiniBooNE $\mathrm{CC} 1 \pi^{+}$data sample, which favors a higher normalization and different $Q^{2}$ shape than that given by the NUANCE generator.

The method used to estimate the uncertainties due to the underlying neutrino flux prediction and detector model is identical to that used in previous MiniBooNE results $[9,14]$. For the flux, the dominant uncertainty is in the production of $\pi^{+/-}$from $\mathrm{pBe}$ interactions. Light and particle production and propagation in the MiniBooNE detector is modeled using a GEANT3 [15] based simulation, which was tuned using MiniBooNE and external data.

For the disappearance search, systematic uncertainties are included for the underlying neutrino flux prediction, neutrino interaction cross section, and detector predictions. These uncertainties produce correlated errors between $E_{\nu}^{Q E}$ bins that are included by developing a covariance matrix in the same manner as for previous oscillation results[2, 14]. This covariance matrix has pure normalization and shape-only components that can be separated. For the shape-only disappearance search, just the shape-only covariance matrix is used.

The disappearance search uses the Pearson's $\chi^{2}$ test to determine allowed regions in the $\Delta m^{2}-\sin ^{2} 2 \theta$ plane. The $\chi^{2}$ is calculated from a comparison of the data, $d_{i}$, in $E_{\nu}^{Q E}$ bin $i$ to a prediction $p_{i}\left(\Delta m^{2}, \sin ^{2} 2 \theta\right)$ with a two-flavor $\nu_{\mu} \rightarrow \nu_{x}$ disappearance hypothesis:

$$
\chi^{2}=\sum_{i, j}^{N b i n s}\left(d_{i}-N p_{i}\right) M_{i j}{ }^{-1}\left(d_{j}-N p_{j}\right)
$$

where $M_{i j}$ is the shape-only error matrix, and $N$ normalizes the prediction to the total number of data events. All neutrino events in the prediction, including the $\mathrm{CC} 1 \pi^{+}$ background events, are allowed to oscillate in the fit based on the true neutrino energy and distance traveled. The $90 \%$ CL limit corresponds to $\chi^{2}>23.5$ for 16 bins The sensitivity is a fit to an unoscillated prediction including all statisical and systematic uncertainties.

The top plot of Fig.1 (Fig.2) shows $E_{\nu}^{Q E}$ after selection cuts for the neutrino (antineutrino) data and the null prediction with diagonal elements of the error matrix. Though the diagonal elements of the error matrix are substantial, the correlations between energy bins are large so it is hard to interpret the sensitivity to changes due to neutrino oscillations from Fig.1 and Fig.2. The dominant systematics arise from uncertainties in the neutrino flux and CCQE cross section; uncertainties at low energy are larger because of the substantial $\mathrm{CC} 1 \pi^{+}$background and uncertainties in the CCQE cross section in

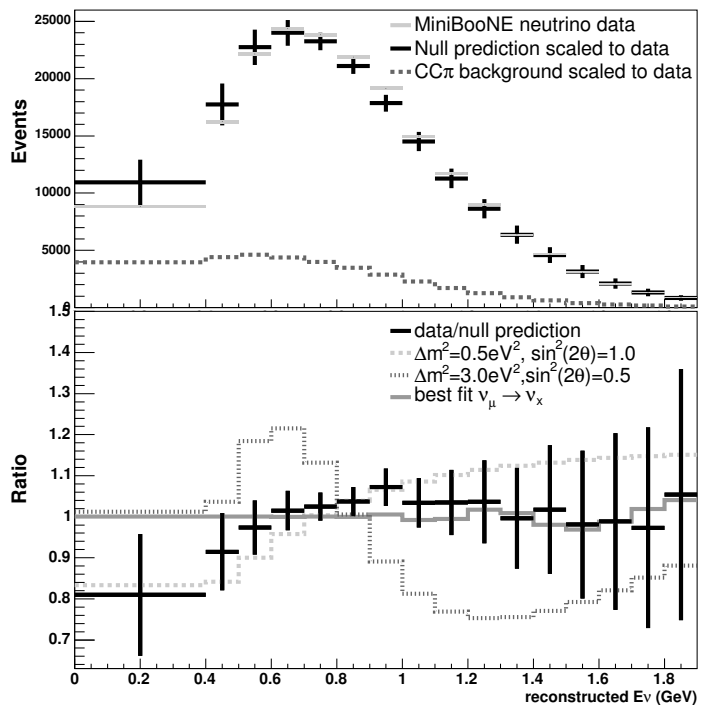

FIG. 1: The top plot shows the $E_{\nu}^{Q E}$ distribution for neutrino data (grey) with statistical error rectangles (thickness of line indicates size of statistical error), and null hypothesis (black). Attached to the prediction are the diagonal elements of the shape error matrix. CC1 $\pi$ background is shown in dashed line. The bottom plot shows the ratio of data to: null hypothesis (black), $\Delta m^{2}=0.5 \mathrm{eV}^{2}, \sin ^{2} 2 \theta=1.0$ disappearance (dashed line), $\Delta m^{2}=3.0 e V^{2}, \sin ^{2} 2 \theta=0.5$ disappearance (dotted line) and for the minimum $\chi^{2}=12.72$ (16 dof) hypothesis $\Delta m^{2}=17.5 \mathrm{eV}^{2}, \sin ^{2} 2 \theta=0.16$ (solid line).

this region.

The bottom plot in Fig.1 (Fig.2) shows the ratio of data to the null hypothesis and three oscillation scenarios. The shape distortion for $\Delta m^{2}=0.5 \mathrm{eV}^{2}$ is very different from $\Delta m^{2}=3.0 \mathrm{eV}^{2}$. The $\chi^{2}$ therefore changes rapidly as a function of $\Delta m^{2}$, resulting in rapid changes in the 90\%CL sensitivity and limit curves (Fig.3) for small differences in $\Delta m^{2}$. Similar features are also seen in previous disappearance analyses [7, 8].

The $\bar{\nu}_{\mu}$ disappearance analysis is the same as the $\nu_{\mu}$ analysis, except that only the $\bar{\nu}_{\mu}$ events are allowed to oscillate in the fit and the $\nu_{\mu}$ events are kept fixed. This determines the limit on a model where the $\bar{\nu}_{\mu}$ can oscillate but the $\nu_{\mu}$ cannot. A model where both $\nu_{\mu}$ and $\bar{\nu}_{\mu}$ oscillate with equal oscillation probability vs energy would produce a limit very similar to Fig.3.

During antineutrino data taking, two absorber plates fell vertically into the decay volume at $25 \mathrm{~m}$ and were later removed, creating three distinct data taking periods with zero, one, or two absorbers in the beamline. The event rate is reduced by $13 \%$ for one plate and by an additional $7 \%$ for the second plate. Approximately $15 \%$ of the antineutrino data has one absorber plate inserted, and $15 \%$ 


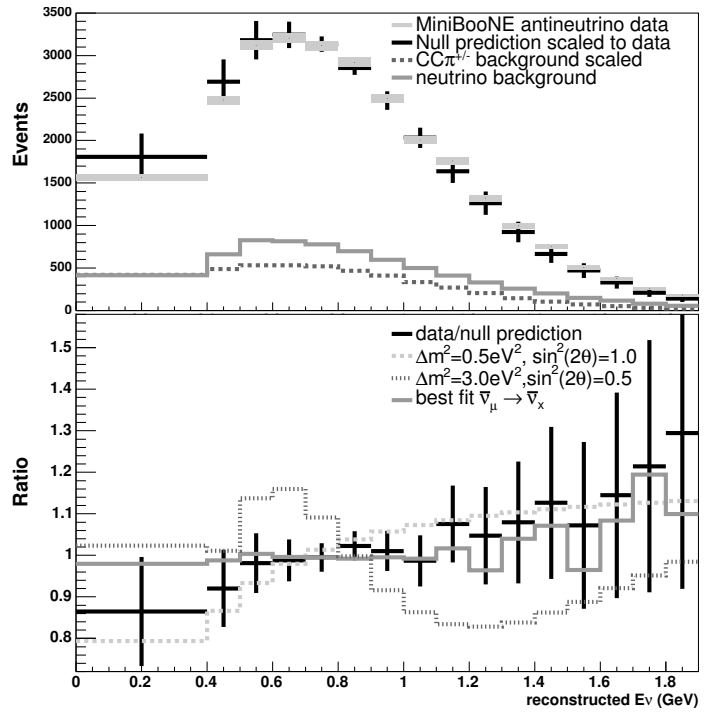

FIG. 2: The top plot shows the $E_{\nu}^{Q E}$ distribution for antineutrino data with the same convention as Fig.1. Additionally, neutrino events are light grey. The bottom plot shows the ratio of data to oscillation scenarios with the same convention as Fig.1. Minimum $\chi^{2}=5.43$ (16 dof) corresponds to $\Delta m^{2}=31.3 e V^{2}, \sin ^{2} 2 \theta=0.96$.

has two absorber plates inserted. Because the changes to the beamline are understood, a separate simulation was run with the appropriate number of absorber plates in the beamline. The $\chi^{2}$ of the null hypothesis is 13.7, 8.2, 15.2 (16 dof) for the zero, one, and two absorber plate data respectively. Fig. 4 shows the 90\% CL sensitivity and limit curves for the antineutrino disappearance fit to all antineutrino data; the limit curves to the individual absorber data periods are all consistent with the total.

In summary, MiniBooNE observes no evidence for $\nu_{\mu}$ or $\bar{\nu}_{\mu}$ disappearance in the $\Delta m^{2}$ region of a few $\mathrm{eV}^{2}$. The best fits for $3+2$ sterile neutrino models in Ref[5] are consistent with MiniBooNE $\nu_{\mu}$ and $\bar{\nu}_{\mu}$ data, but $\nu_{\mu}$ data rules out the best fit point of global fit to MiniBooNE $\nu_{e}$ data in $\operatorname{Ref}[4]$ at $90 \%$ CL with $\chi^{2}=24.7$ (16 dof). This is the first test of $\bar{\nu}_{\mu}$ disappearance between $\Delta m^{2}=$ $0.1-10 \mathrm{eV}^{2}$.

We acknowledge the support of Fermilab, the Department of Energy, and the National Science Foundation. We thank Los Alamos National Laboratory for LDRD funding. We also acknowledge the use of Condor software in the analysis of the data.

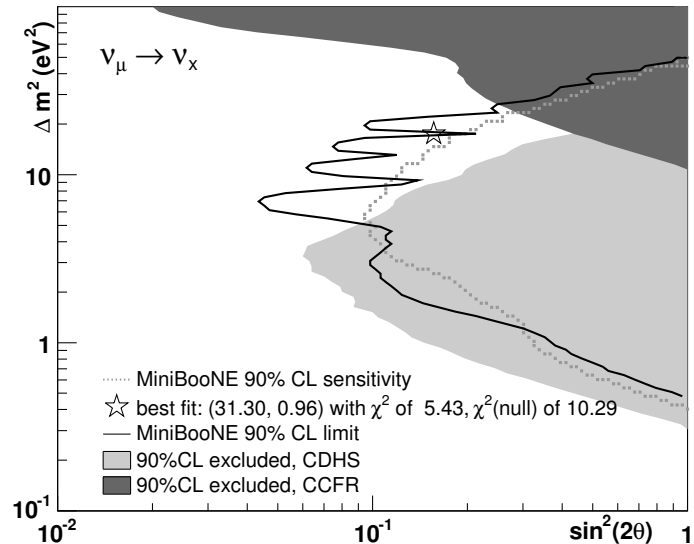

FIG. 3: The sensitivity (dashed line) and limit (solid line) for 90\% CL for neutrino disappearance in MiniBooNE. Previous limits by CDHS (light grey) and CCFR (dark grey) are also shown. The $\chi^{2}$ of the null hypothesis is 17.78 (16 dof).

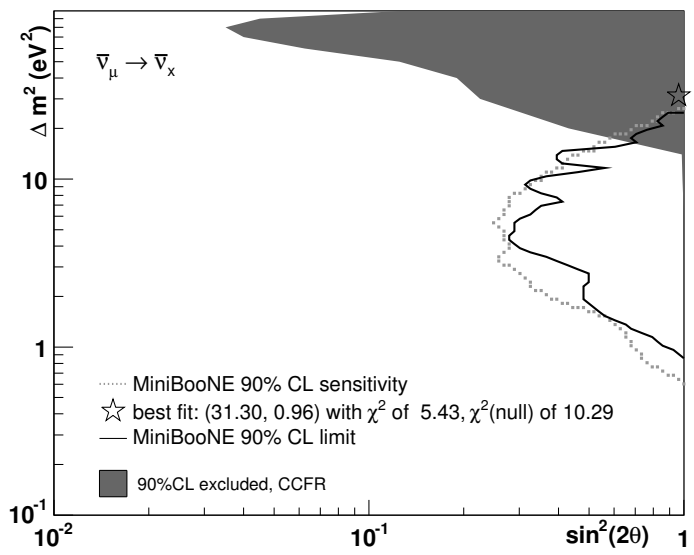

FIG. 4: The sensitivity (dashed line) and limit (solid line) for $90 \%$ CL for antineutrino disappearance in MiniBooNE. Previous limits by CCFR (dark grey) are also shown. The $\chi^{2}$ of the null hypothesis is 10.29 (16 dof).

[1] C. Athanassopoulos et al., Phys. Rev. Lett. 75, 2650 (1995); C. Athanassopoulos et al.,Phys. Rev. Lett. 77, 3082 (1996); C. Athanassopoulos et al.,Phys. Rev. Lett. 81, 1774 (1998); A. Aguilar et al., Phys. Rev. D 64 112007 (2001)

[2] A. Aguilar-Arevalo et al., Phys. Rev. Lett. 98, 231801 (2007).

[3] H. Paes, S. Pakvasa, T. J. Weiler, Phys. Rev. D 72, 095017 (2005)

[4] M. Maltoni and T. Schwetz, Phys. Rev. D 72, 093005 
(2007).

[5] G. Karagiorgi, A. Aguilar-Arevalo, J. M. Conrad, M. H. Shaevitz, K. Whisnant, M. Sorel and V. Barger, Phys. Rev. D 75, 013011 (2007).

[6] A. E. Nelson and J. Walsh, Phys. Rev. D 77, 033001 (2008).

[7] I. E. Stockdale et al., Phys. Rev. Lett. 52, 1384 (1984).

[8] F. Dydak et al., Phys. Lett. B 134, 281 (1984).

[9] A. Aguilar-Arevalo et al., [arXiv:0806.1449] accepted by Phys. Rev. D.

[10] S. Agostinelli et al., Nucl. Instrum. Meth. A506, 250 (2003).

[11] A. Aguilar-Arevalo et al., Nucl. Instrum. Meth. A599,
28 (2009).

[12] D. Casper, Nucl. Phys. Proc. Suppl. 112, 161 (2002).

[13] A. A. Aguilar-Arevalo et al. Phys. Rev. Lett. 100, 032301 (2008)

[14] A. Aguilar-Arevalo et al., [arXiv:0812.2243], accepted by Phys. Rev. Lett.

[15] CERN Program Library Long Writeup W5013 (1993).

[16] A. Bodek, S. Avvakumov, R. Bradford and H. Budd, J. Phys. Conf. Ser. 110, 082004 (2008) [arXiv:0709.3538].

[17] X. Espinal and F. Sanchez, AIP Conf. Proc. 967, 117 (2007).

[18] R. Gran et al. Phys. Rev. D 74, 052002 (2006) 


\section{Bibliography}

[1] [ALEPH Collaboration and DELPHI Collaboration and L3 Collaboration and ], Phys. Rept. 427, 257 (2006) [arXiv:hep-ex/0509008].

[2] V. M. Lobashev et al., Phys. Lett. B 460, 227 (1999).

[3] K. Assamagan et al., Phys. Rev. D 53, 6065 (1996).

[4] R. Barate et al. [ALEPH Collaboration], Eur. Phys. J. C 2, 395 (1998).

[5] T. Katori, "A measurement of the muon neutrino charged current quasielastic interaction and a test of Lorenz violation with the MiniBooNE experiment"

[6] B. T. Cleveland et al., Astrophys. J. 496, 505 (1998).

[7] J. N. Abdurashitov et al. [SAGE Collaboration], Phys. Rev. C 60, 055801 (1999) [arXiv:astro-ph/9907113].

[8] W. Hampel et al. [GALLEX Collaboration], Phys. Lett. B 447, 127 (1999).

[9] S. Fukuda et al. [Super-Kamiokande Collaboration], Phys. Lett. B 539, 179 (2002) [arXiv:hep-ex/0205075].

[10] Q. R. Ahmad et al. [SNO Collaboration], Phys. Rev. Lett. 87, 071301 (2001) [arXiv:nucl-ex/0106015].

[11] Q. R. Ahmad et al. [SNO Collaboration], Phys. Rev. Lett. 89, 011301 (2002) [arXiv:nucl-ex/0204008].

[12] S. N. Ahmed et al. [SNO Collaboration], Phys. Rev. Lett. 92, 181301 (2004) [arXiv:nucl-ex/0309004]. 
[13] K. Eguchi et al. [KamLAND Collaboration], Phys. Rev. Lett. 90, 021802 (2003) [arXiv:hep-ex/0212021].

[14] T. Araki et al. [KamLAND Collaboration], Phys. Rev. Lett. 94, 081801 (2005) [arXiv:hep-ex/0406035].

[15] Y. Fukuda et al. [Kamiokande Collaboration], Phys. Lett. B 335, 237 (1994).

[16] M. H. Ahn et al. [K2K Collaboration], Phys. Rev. D 74, 072003 (2006) [arXiv:hepex/0606032].

[17] D. G. Michael et al. [MINOS Collaboration], Phys. Rev. Lett. 97, 191801 (2006) [arXiv:hep-ex/0607088].

[18] C. Athanassopoulos et al., Phys. Rev. Lett. 75, 2650 (1995); C. Athanassopoulos et al., Phys. Rev. Lett. 77, 3082 (1996); C. Athanassopoulos et al., Phys. Rev. Lett. 81, 1774 (1998); A. Aguilar et al., Phys. Rev. D 64, 112007 (2001).

[19] A. Aguilar-Arevalo et al., Phys. Rev. Lett. 98, 231801 (2007).

[20] I. E. Stockdale et al., Phys. Rev. Lett. 52, 1384 (1984).

[21] F. Dydak et al., Phys. Lett. B 134, 281 (1984).

[22] M. Sorel, J. M. Conrad and M. Shaevitz, Phys. Rev. D 70, 073004 (2004) [arXiv:hep$\mathrm{ph} / 0305255]$.

[23] G. Karagiorgi, A. Aguilar-Arevalo, J. M. Conrad, M. H. Shaevitz, K. Whisnant, M. Sorel and V. Barger, Phys. Rev. D 75, 013011 (2007).

[24] M. Maltoni, J. Phys. Conf. Ser. 110, 082011 (2008) [arXiv:0711.2018 [hep-ph]].

[25] M. Maltoni and T Schwetz Phys. Rev. D. 76, 093005 (2007) [arXiv:0705.0107 [hep$\mathrm{ph}]$.

[26] S. Antusch, C. Biggio, E. Fernandez-Martinez, M. B. Gavela and J. Lopez-Pavon, JHEP 0610, 084 (2006) [arXiv:hep-ph/0607020]. 
[27] H. Pas, S. Pakvasa and T. J. Weiler, Phys. Rev. D 72, 095017 (2005) [arXiv:hep$\mathrm{ph} / 0504096]$.

[28] S. Palomares-Ruiz, S. Pascoli and T. Schwetz, JHEP 0509, 048 (2005) [arXiv:hep$\mathrm{ph} / 0505216]$.

[29] A. E. Nelson and J. Walsh, Phys. Rev. D 77, 033001 (2008) [arXiv:0711.1363 [hep-ph]].

[30] Accelerator Division of Fermilab has a series of "rookie" books which describe each section of the accelerator in detail. See http://wwwbdnew.fnal.gov/operations/rookie_books/rbooks.html for the Booster rookie book.

[31] "8 GeV Beam Technical Design Report". http://wwwboone.fnal.gov/publicpages/8gevtdr 2.0.ps.gz

[32] S. E. Kopp, Phys. Rept. 439, 101 (2007) [arXiv:physics/0609129].

[33] S. Yamamoto et al., IEEE Trans. Nucl. Sci. 52, 2992 (2005).

[34] K. Nitta et al., Nucl. Instrum. Meth. A 535, 147 (2004) [arXiv:hep-ex/0406023].

[35] K. Hiraide, "A Study of Charged Current Single Charged Pion Productions on Carbon in a Few-GeV Neutrino Beam," CITATION = FERMILAB-THESIS-2009-02;

[36] M. Hasegawa et al. [K2K Collaboration], Phys. Rev. Lett. 95, 252301 (2005) [arXiv:hep-ex/0506008].

[37] A. Rodriguez et al. [K2K Collaboration], Phys. Rev. D 78, 032003 (2008) [arXiv:0805.0186 [hep-ex]].

[38] S. Buontempo et al., Nucl. Instrum. Meth. A 349, 70 (1994).

[39] A. Aguilar-Arevalo et al., Nucl. Instrum. Meth. A599, 28 (2009).

[40] E. Hawker, G. Garvey, S. Freudenstein, A. Lathrop, G. Mills, “Oil Attenuation Measurements with the 'Bama Oil Tester", MiniBooNE internal document 
[41] S. Brice, B. Fleming, P. Meyers, R. Patterson, H. Tanaka, M. Wasko, “BooNE Detector Monte Carlo Baseline Parameters and Variants", MiniBooNE internal document

[42] A. O. Bazarko, P. D. Meyers, R. B. Patterson, F. C. Shoemaker, H. A. Tanaka, “Studies of Scattering in Marcol 7 Mineral Oil", MiniBooNE internal document

[43] D. Toptygin, "Time-Resolved Fluorecence of MiniBooNE Mineral Oil” , MiniBooNE internal document

[44] A. Aguilar-Arevalo et al., arXiv:0806.1449 [hep-ex], accepted by Phys. Rev. D.

[45] S. Agostinelli et al., Nucl. Instrum. Meth. A506, 250 (2003).

[46] J. R. Sanford and C. L. Wang, Brookhaven National Laboratory, AGS internal reports 11299 and 11479 (1967) (unpublished)

[47] M. G. Catanesi et al. [HARP Collaboration], Eur. Phys. J. C52, 29 (2007).

[48] D. W. Schmitz, "A measurement of hadron production cross sections for the simulation of accelerator neutrino beams and a search for $\nu_{\mu}$ to $\nu_{e}$ oscillations in the $\delta m^{2}$ about equals $1-e V^{2}$ region," CITATION = FERMILAB-THESIS-2008-26;

[49] I. Chemakin et al. [E910 Collaboration], Phys. Rev. C 77, 015209 (2008) [Erratum-ibid. C 77, 049903 (2008)] [arXiv:0707.2375 [nucl-ex]].

[50] A. S. Carroll et al., Phys. Rev. C 14, 635 (1976).

[51] D. Ashery, I. Navon, G. Azuelos, H. K. Walter, H. J. Pfeiffer and F. W. Schleputz, Phys. Rev. C 23, 2173 (1981).

[52] B. W. Allardyce et al., Nucl. Phys. A 209, 1 (1973).

[53] B. M. Bobchenko et al., Sov. J. Nucl. Phys. 30, 805 (1979) [Yad. Fiz. 30, 1553 (1979)].

[54] T. Abbott et al., Phys. Rev. D45, 3906 (1992);

[55] J. V. Allaby et al., CERN 70-12 (1970);

[56] D. Dekkers et al., Phys. Rev. 137, B962 (1965); 
[57] T. Eichten et al., Nucl. Phys. B44, 333 (1972);

[58] A. Aleshin et al., ITEP-77-80 (1977);

[59] I. A. Vorontsov et al., ITEP-88-11 (1988).

[60] C. Amsler et al. [Particle Data Group], Phys. Lett. B 667, 1 (2008).

[61] D. Allasia et al., Nucl. Phys. B383, 285 (1990).

[62] N. J. Baker et al., Phys. Rev. D 23, 2499 (1981).

[63] N. J. Baker, P. L. Connolly, S. A. Kahn, M. J. Murtagh, R. B. Palmer, N. P. Samios and M. Tanaka, Phys. Rev. D 25, 617 (1982).

[64] C. Baltay et al., Phys. Rev. Lett. 44, 916 (1980).

[65] S. J. Barish et al., Phys. Rev. D 16, 3103 (1977).

[66] S. J. Barish et al., Phys. Rev. D 19, 2521 (1979).

[67] D. S. Baranov et al., Phys. Lett. 81B, 255 (1979).

[68] S. Bonetti et al., Nuovo Cimento A38, 260 (1977).

[69] S. Kitagaki et al., Phys. Rev. D28, 436 (1983).

[70] S. V. Belikov et al., Z. Phys. A 320, 625 (1985).

[71] J. P. Berge et al., Z. Phys. C 35, 443 (1987).

[72] J. Brunner et al., Z. Phys. C 45, 551 (1990).

[73] D. C. Colley et al., Z. Phys. C 2, 187 (1979).

[74] S. Ciampolillo et al., Phys. Lett. 84B, 281 (1979).

[75] D. MacFarlane et al., Z. Phys. C 26, 1 (1984).

[76] J. G. Morfin et al., Phys. Lett. 104B, 235 (1981).

[77] A. .S. Vovenko et al., Sov. J. Nucl. Phys. 30, 527 (1979). 
[78] N. Armenise et al., Nucl. Phys. B 152, 365 (1979).

[79] A. .E. Asratian et al., Sov. J. Nucl. Phys. 39, 392 (1984).

[80] G. Fanourakis et al., Phys. Rev. D21, 562 (1980).

[81] D. Casper, Nucl. Phys. Proc. Suppl. 112, 161 (2002).

[82] C. H. Llwellyn-Smith, Phys. Rep. 3:261 (1972)

[83] H. Budd, A. Bodek and J. Arrington, arXiv:hep-ex/0308005.

[84] M. K. Jones et al. [Jefferson Lab Hall A Collaboration], Phys. Rev. Lett. 84, 1398 (2000) [arXiv:nucl-ex/9910005].

[85] O. Gayou et al. [Jefferson Lab Hall A Collaboration], Phys. Rev. Lett. 88, 092301 (2002) [arXiv:nucl-ex/0111010].

[86] A. F. Krutov and V. E. Troitsky, Eur. Phys. J. A 16, 285 (2003) [arXiv:hep-ph/0202183].

[87] S. Galster, H. Klein, J. Moritz, K. H. Schmidt, D. Wegener and J. Bleckwenn, Nucl. Phys. B 32, 221 (1971).

[88] C. W. Walter, K. McConnel and M. Sakuda, (NuFact03 proceedings) AIP Conf. Proc. 721, 247 (2004)

[89] R. A. Smith and E. J. Moniz, Nucl. Phys. B 43, 605 (1972) [Erratum-ibid. B 101, 547 (1975)].

[90] O. Benhar and D. Meloni, Nucl. Phys. A 789, 379 (2007) [arXiv:hep-ph/0610403].

[91] A. Bodek, S. Avvakumov, R. Bradford, and H. Budd, J. Phys. Conf. Ser. 110, 082004 (2008).

[92] X. Espinal and F. Sanchez, AIP Conf. Proc. 967, 117 (2007).

[93] R. Gran et al., Phys. Rev. D 74, 052002 (2006).

[94] A. A. Aguilar-Arevalo et al., Phys. Rev. Lett. 100, 032301 (2008). 
[95] V. Lyubushkin et al. [NOMAD Collaboration], arXiv:0812.4543 [hep-ex].

[96] D. Rein and L. M. Sehgal, Annals Phys. 133, 79 (1981).

[97] Jaroslaw Nowak, private communication.

[98] I. Navon et al., Phys. Rev. Lett. 52, 105 (1984).

[99] D. Ashery, I. Navon, G. Azuelos, H. K. Walter, H. J. Pfeiffer and F. W. Schleputz, Phys. Rev. C 23, 2173 (1981).

[100] M. K. Jones et al., Phys. Rev. C 48, 2800 (1993).

[101] R. D. Ransome et al., Phys. Rev. C 45, 509 (1992).

[102] A. S. Clough et al., Nucl. Phys. B 76, 15 (1974).

[103] C. Wilkin et al., Nucl. Phys. B 62, 61 (1973).

[104] CERN Program Library Long Writeup W5013 (1993).

[105] P. Meyers, "A new mu- capture model for the BooNE detector Monte Carlo", MiniBooNE internal document

[106] C. Zeitnitz and T. A. Gabriel, Nucl. Instrum. Meth. A 349, 106 (1994).

[107] H. Maesaka, Ph.D thesis, Kyoto University (2005)

[108] R. B. Patterson, E. M. Laird, Y. Liu, P. D. Meyers, I. Stancu and H. A. Tanaka, “The extended-track reconstruction for MiniBooNE," arXiv:0902.2222 [hep-ex].

[109] A. A. Aguilar-Arevalo, "An improved Neutrino Oscillations Analysis of the MiniBooNE Data," CITATION = FERMILAB-THESIS-2008-01;

[110] T. Katori, R. Tayloe, G. Zeller, “Update on the MiniBooNE numu QE Analysis”, MiniBooNE internal document

[111] M. Sorel, "Search for Sterile Neutrinos Using the MiniBooNE Beam" Columbia University thesis 
[112] I. Stancu, “Maximum Likelihood Event Reconstruction for BooNE: Point like particles in a perfect detector", MiniBooNE internal document

[113] Y. Liu, I. Stancu, "The MiniBooNE Charge Likelihoods and Light Scattering in Michel Electron Events", MiniBooNE internal document

[114] T. Suzuki et all, Phys. Rev. C 35 (1987) 2212-2224

[115] L. Devroye,“Non Uniform Random Variate Generation”, Springer-Verlag, NY, 1986. http:/ /cg.scs.carleton.ca/luc/rnbookindex.html

[116] Y. Declais et al., Nucl. Phys. B 434, 503 (1995).

[117] M. Apollonio et al. [CHOOZ Collaboration], Eur. Phys. J. C 27, 331 (2003) [arXiv:hepex/0301017].

[118] M. Shaevitz, "Separating Components of Error Matrices", MiniBooNE internal document 